\title{
Numerical Simulation of the Interaction of Wingtip Vortices and Engine Jets in the Near Field
}

\author{
Von der Fakultät für Maschinenwesen \\ der Rheinisch-Westfälischen Technischen Hochschule Aachen \\ zur Erlangung des akademischen Grades eines \\ Doktors der Ingenieurwissenschaften \\ genehmigte Dissertation \\ vorgelegt von
Diplom-Ingenieur Ehab Mahmoud Aziz Mohamed Fares aus El-Menia, Ägypten

Berichter: Universitätsprofessor Dr.-Ing. Wolfgang Schröder Universitätsprofessor Dr.-Ing. Dieter Jacob

Tag der mündlichen Prüfung: 18.11.2002 

To my parents 



\section{Acknowledgements}

This thesis was written during my work as a research scientist at the Chair of Fluid Mechanics and Institute of Aerodynamics $A I A$ at the Aachen University of Technology $R W T H$ Aachen, Germany. The research has been funded by the Deutsche Forschungsgemeinschaft $D F G$ through the collaborative research program $S F B-401$.

First, I would like to express my gratitude to my advisor Professor Wolfgang Schröder for his guidance, encouragement and advice. The freedom and support he offered me during all times are greatly appreciated and represent a key factor in the success of this work. I would also like to thank the members of my committee Professor Jacob and Professor Renz.

In addition, I would like to thank Dr.-Ing. Matthias Meinke and Dr.-Ing. Andreas Henze as representatives of my colleagues at the Institute, who supported my work directly through various discussions or indirectly by formulating my research objective as a challenge for the available methods. I also appreciate the collaboration with the former students Dipl.-Ing. Wolfgang Birkenstock and cand.-Ing. Michael Hopp. Furthermore, the fruitful discussions and the long friendship with Dr. Erol Özger deserve a special mention.

I cannot express enough gratitude to my mother and my father for their continuous and unquestioning encouragement to my study. Without their solid belief in the role of education and the support they offered me at different stages of my life the path to this thesis would have been impossible. Therefore, this work is dedicated to them.

Aachen, November 2002.

Ehab M. A. M. Fares 



\section{Contents}

Abstract/Übersicht . . . . . . . . . . . . . . . . . . . iii

Nomenclature ....................... . . . .

1 Introduction $\quad 1$

2 Mathematical Model $\quad 7$

2.1 The Navier-Stokes Equations . . . . . . . . . . . . . . . 8

2.2 The Time-Averaged Navier-Stokes Equations . . . . . . . . . . . . . 10

2.3 Initial and Boundary Conditions . . . . . . . . . . . . . . . . . . . 11

2.3.1 Initial Conditions . . . . . . . . . . . . . . . . . 11

2.3.2 Boundary Conditions at Solid Walls . . . . . . . . . . . . . 11

2.3.3 Boundary Conditions at Inflow/Outflow . . . . . . . . . . . . 12

3 Turbulence Modeling 13

3.1 One-Equation Turbulence Models . . . . . . . . . . . . . . . . . . 15

3.1.1 The Spalart-Allmaras Model . . . . . . . . . . . . . . . 16

3.1.2 A New One-Equation Turbulence Model . . . . . . . . . . . . . 17

3.2 Wall Distance Approximation . . . . . . . . . . . . . . . . . 24

4 Method of Solution $\quad 31$

4.1 Finite Volume Method . . . . . . . . . . . . . . . . . 31

4.1 .1 Boundary-Fitted Structured Grid . . . . . . . . . . . . . . 32

4.1.2 Node Centered Scheme . . . . . . . . . . . . . . . . 33

4.1.3 Multiblock Arrangement and Parallelization . . . . . . . . . . . 33

4.2 Spatial Discretization . . . . . . . . . . . . . . . . . . 35

4.2.1 Discretization of the Advective Terms . . . . . . . . . . . . . 35

4.2.2 Discretization of the Dissipative Terms . . . . . . . . . . . . 36

4.2.3 Discretization of the Source Terms . . . . . . . . . . . . . 37

4.3 Temporal Discretization . . . . . . . . . . . . . . . . . 37

4.4 Acceleration to Steady State . . . . . . . . . . . . . . . . . . . 39

4.4.1 Local Time Stepping . . . . . . . . . . . . . . . . . . . . . . . . 39

4.4.2 Rational Runge-Kutta Integration Scheme . . . . . . . . . . . 39 
4.4 .3 Implicit Residual Smoothing . . . . . . . . . . . . . . . . . 40

4.4 .4 Multigrid Method . . . . . . . . . . . . . . . . . 40

4.5 Initial and Boundary Conditions . . . . . . . . . . . . . . . . 41

4.5 .1 Initial Conditions . . . . . . . . . . . . . . . . . 41

4.5 .2 Boundary Conditions . . . . . . . . . . . . . . . . 41

4.5.3 Boundary Conditions for the Engine Jet . . . . . . . . . . 46

5 Results and Discussion $\quad 49$

5.1 Validation . . . . . . . . . . . . . . . . . . . . . . . . . 49

5.1 .1 Turbulent Free Shear Flows . . . . . . . . . . . . . . . 50

5.1 .2 Isolated Vortex . . . . . . . . . . . . . . . . . . . 54

$5.1 .3 \quad$ Flat Plate Boundary Layer . . . . . . . . . . . . . . . . . . . . 59

5.1 .4 Aerodynamic Flows . . . . . . . . . . . . . . . . . . 67

5.1 .5 Wake of Airbus A300 . . . . . . . . . . . . . . 71

5.2 Rectangular Wing with Engine Jets . . . . . . . . . . . . . . 76

5.2 .1 Flow Around the Rectangular Wing . . . . . . . . . . . . 77

5.2 .2 Wake Flow . . . . . . . . . . . . . . . . . . . 88

6 Conclusions 109

$\begin{array}{ll}\text { References } & 111\end{array}$

$\begin{array}{ll}\text { Appendix } & 119\end{array}$

A Dimensionless Variables . . . . . . . . . . . . . . . . . . . . . 119

B Averaging Rules . . . . . . . . . . . . . . . . . . . . . . . 120

C Equations in Cartesian Coordinates . . . . . . . . . . . . . . . . 121

D Transformation to Curvilinear Coordinates . . . . . . . . . . . . . . . 124

E Equations in Curvilinear Coordinates . . . . . . . . . . . . . . . . 125

F Discretization of Advective Terms according to the AUSM Scheme . . 127

G Characteristical Form of the Euler Equations . . . . . . . . . . . . 130 


\section{Abstract}

The near wake of a rectangular wing/engine configuration is investigated using numerical simulations. The analysis focuses on the interaction of the engine jet and the wingtip vortex in the near wake. Numerical issues such as discretizations, acceleration techniques and boundary conditions are discussed in detail. A new one-equation turbulence model is derived, which gives more accurate results than other one-equation turbulence models especially for free shear and vortical flows that are essential in the wake flow problem. Several fundamental flows are simulated numerically and the findings are compared with experimental data to validate the newly developed methods and to ensure the general quality of the numerical results.

The thesis analyzes the interaction between the wingtip vortex and the engine jet in the near wake. Physical aspects of the influence of the engine jet on the formation and development of the wingtip vortex at varying thrust and engine positions are documented and discussed on the basis of qualitative and quantitative analyses of the wake flow.

\section{Übersicht}

Der nahe Nachlauf eines Rechteckflügel-/Triebwerkskonfiguration wird mit Hilfe von numerischen Simulationen untersucht. Die Analyse konzentriert sich auf die Interaktion des Triebwerksstrahls mit dem Randwirbel im Nahfeld. Die numerischen Methoden wie Diskretisierungen, Beschleunigungstechniken und Randbedingungen werden ausführlich diskutiert. Ein neues Ein-Gleichungs-Turbulenzmodell, das genauere Ergebnisse als existierende Ein-Gleichungs-Turbulenzmodelle insbesondere für die im Nachlauf vorhandenen Scherschicht- und Wirbelströmungen liefert, wird abgeleitet. Mehrere grundlegende Strömungen werden numerisch simuliert und mit experimentellen Daten verglichen, um die neu entwickelten Methoden zu validieren und die allgemeine Qualität der numerischen Ergebnisse sicherzustellen.

Die Dissertation analysiert die Interaktion zwischen dem Randwirbel und dem Triebwerksstrahl im nahen Nachlauf. Physikalische Aspekte des Einflusses des Triebwerksstrahles auf die Randwirbelbildung und Entwicklung bei unterschiedlichen Triebwerksstrahlgeschwindigkeiten und Triebwerkspositionen sind dokumentiert und anhand von qualitativen und quantitativen Analysen der Nachlaufströmung diskutiert. 


\section{Nomenclature}

Roman Symbols

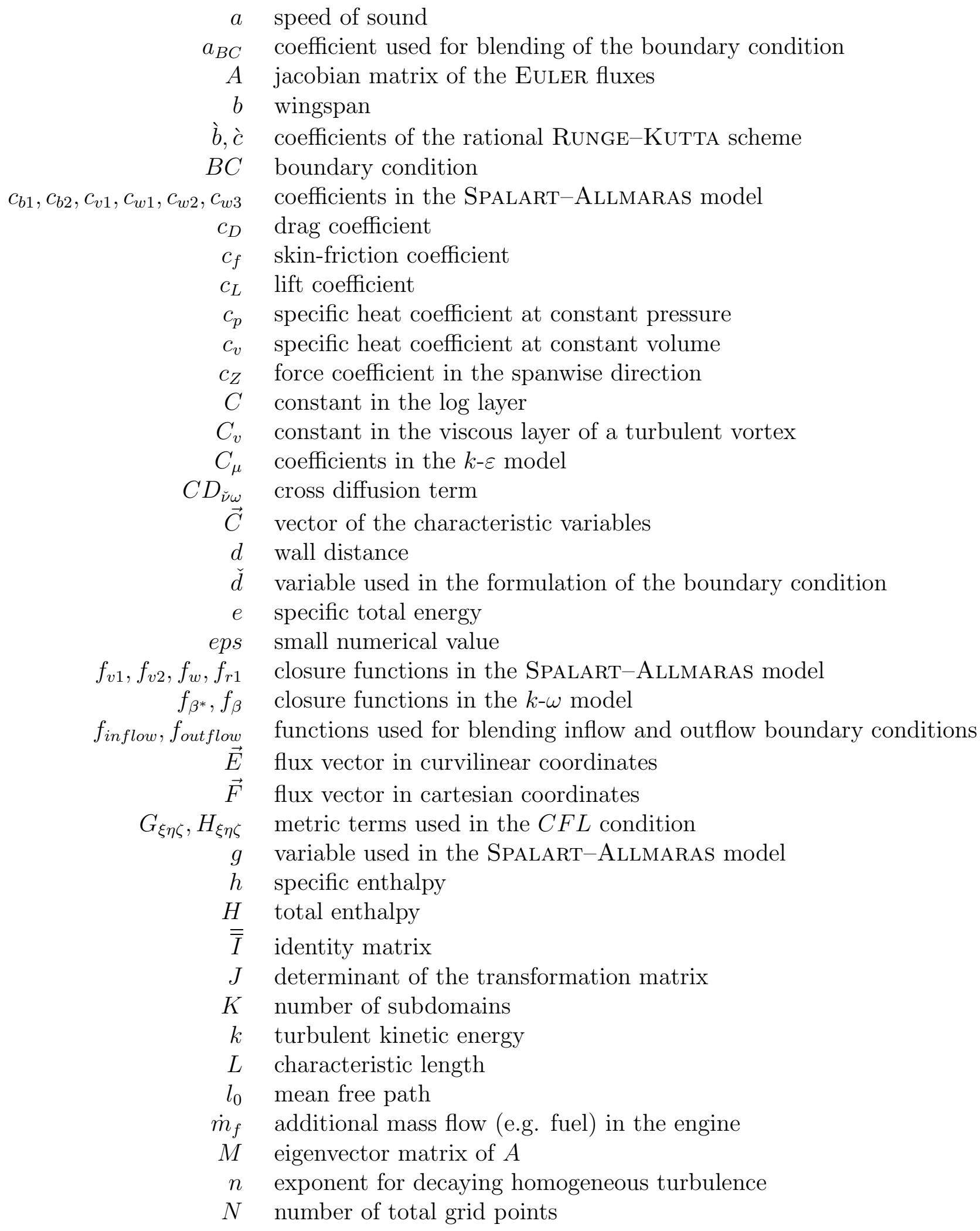




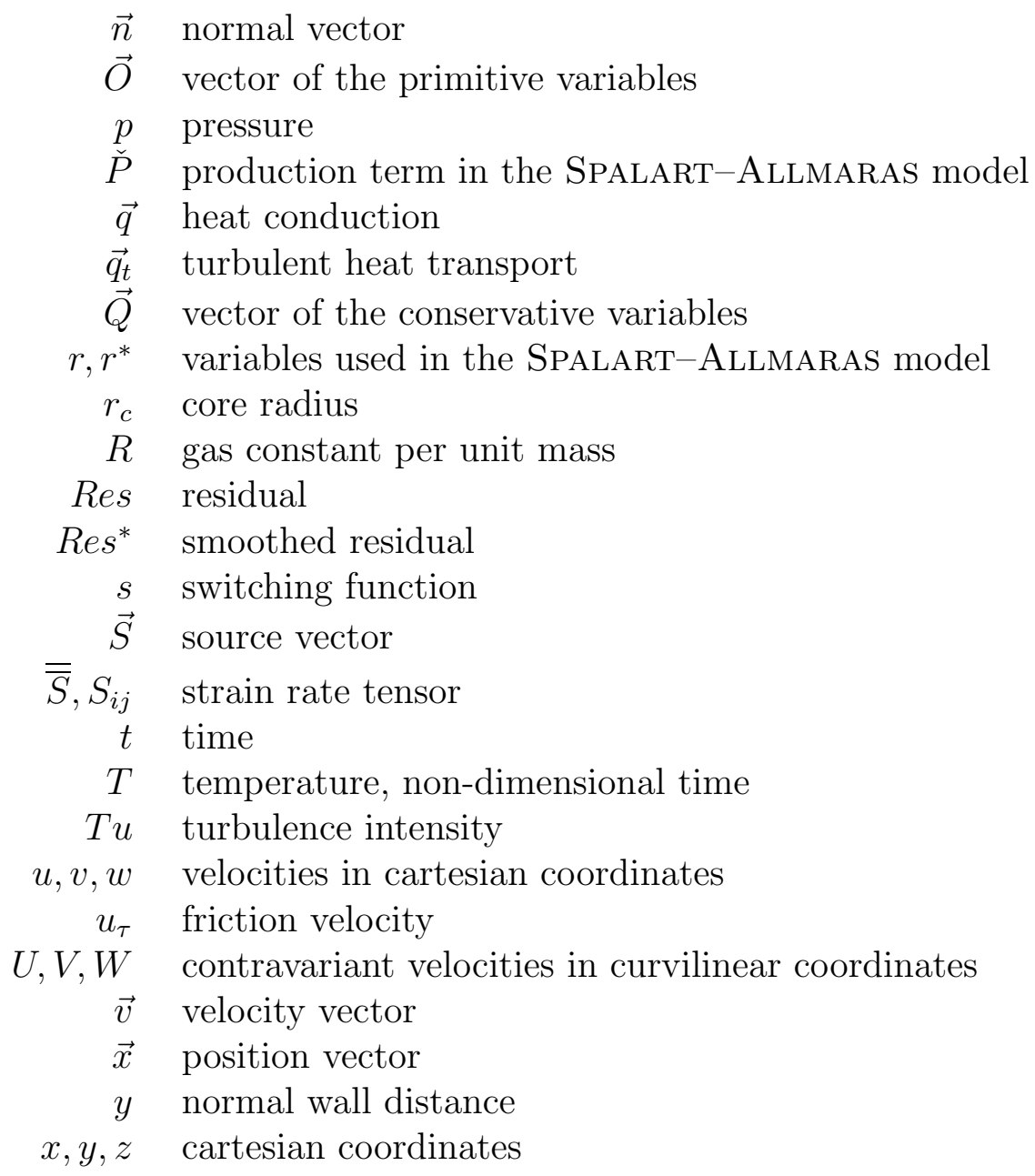

\section{Greek Symbols}

$\alpha \quad$ coefficient used in the $k$ - $\omega$ model, angle of attack

$\breve{\alpha} \quad$ splitting parameter

$\grave{\alpha}$ RunGE-KuTTA coefficient

$\beta_{b}$ BRADSHAW constant

$\beta_{c}^{*}, \beta_{c} \quad$ coefficients used in the $k-\omega$ model

$\beta^{*}, \beta$ modified coefficients used in the $k$ - $\omega$ model

$\epsilon \quad$ specific internal energy

$\epsilon^{*} \quad$ coefficient used in the implicit residual smoothing

$\varepsilon \quad$ turbulent dissipation

$\varepsilon_{\infty / \text { in }}$ ratio used in boundary condition for jet simulation

$\gamma$ ratio of specific heats

$\Gamma$ circulation

$\kappa \quad$ KÁRMÁn constant

$\lambda$ thermal conductivity coefficient

$\lambda_{1,2,3,4,5}$ eigenvalues of $\Lambda$

$\Lambda$ eigenvalue matrix of $A$, aspect ratio 


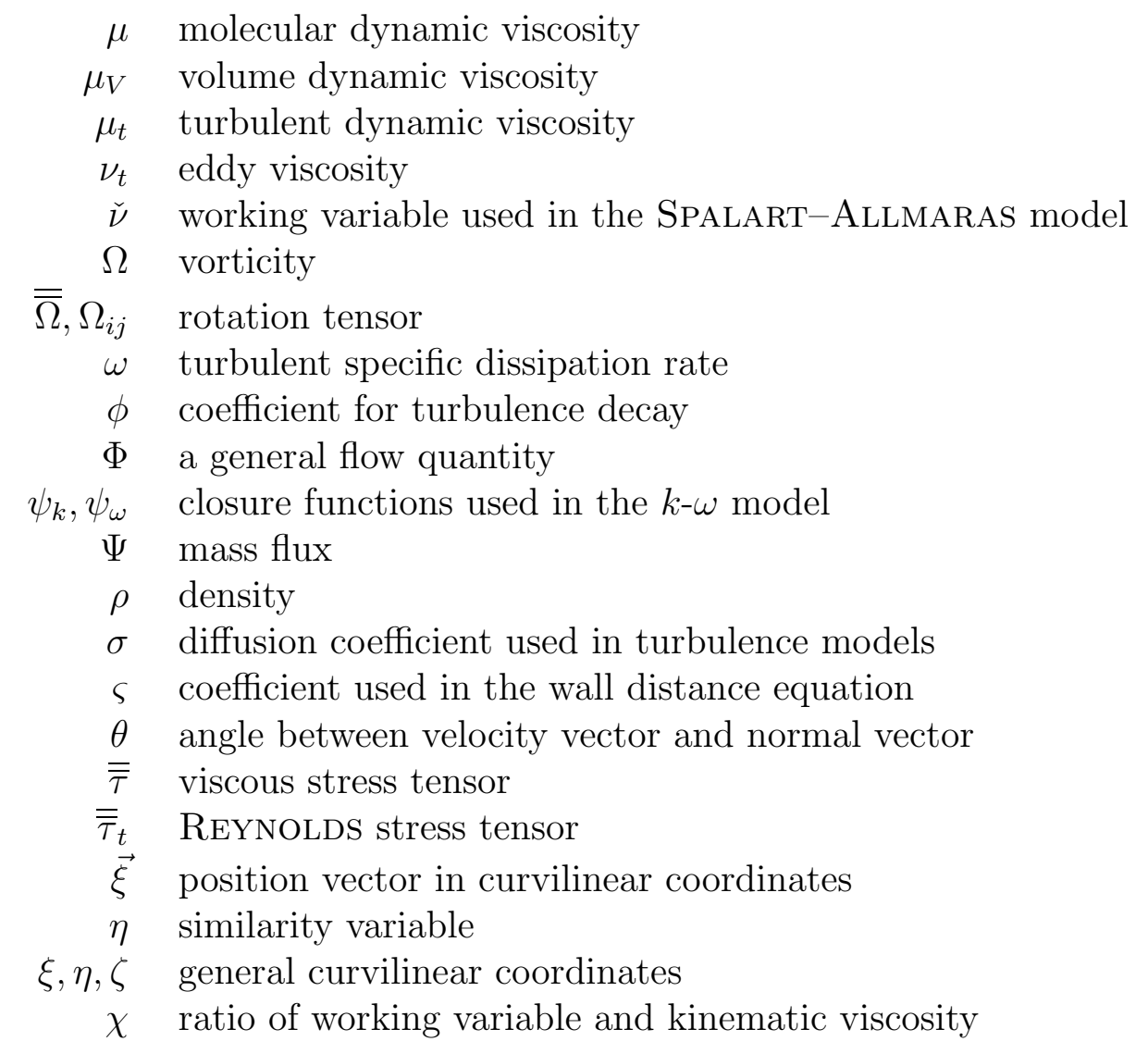

\section{Calligraphic Symbols}

$\mathcal{D}$ distance function

$\mathcal{F}$ distribution function

$\mathcal{G}$ inverse distance function

$\mathcal{L}$ variation of the wave amplitude

$\mathcal{M}$ contravariant local MACH number

$\mathcal{O}(\cdot)$ order of magnitude

$\mathcal{V}$ volume

$\mathcal{S}$ surface

Flow Parameters
$K n \quad$ KNUdSEN number
$M a$ MACH number
$\mathrm{Pr}$ PRANDTL number
Re REYNOLDS number

\section{Operators and Notations}

$$
\begin{aligned}
\frac{D(\cdot)}{D t} & \text { substantial derivative } \\
\max (\cdot, \cdot) & \text { maximum value } \\
\min (\cdot, \cdot) & \text { minimum value }
\end{aligned}
$$




$\begin{aligned} \triangle & \text { delta } \\ \operatorname{diag}(\cdot) & \text { diagonal elements of a matrix } \\ \delta & \text { boundary layer thickness } \\ \Delta & \text { LAPLACE operator } \\ \nabla & \text { nabla operator } \\ \overrightarrow{(\cdot)} & \text { vector } \\ \overrightarrow{(\cdot)} \times \overrightarrow{(\cdot)} & \text { vector product } \\ \overrightarrow{(\cdot)} \cdot \overrightarrow{(\cdot)} & \text { inner product } \\ (\overrightarrow{(\cdot)} \overrightarrow{(\cdot)} & \text { tensor product of two vectors } \\ \overrightarrow{(\cdot)} & \text { REYNOLDS average } \\ \overrightarrow{(\cdot)} & \text { tensor } \\ \tilde{(\cdot)} & \text { FAVRE average } \\ (\cdot) & \text { low REYNOLDS corrected values } \\ (\overrightarrow{(\cdot)} & \text { transformed equation } \\ (\overrightarrow{\cdot T} & \text { transposed vector } \\ \operatorname{trace}(\cdot) & \text { trace of a tensor }\end{aligned}$

The EINSTEIN summation convention is used.

Superscripts

[]$^{+}$sublayer-scaled value

[]$^{+,-}$forward and backward values

[]* values possessing a physical dimension

[]$^{\prime}$ REYNOLDS fluctuation quantity

[]" FAVRE fluctuation quantity

[]$_{\text {critic }}$

[]$^{i, j, k}$

[]$^{(m)}$

[]$^{n}$

critical values

discrete position in grid

discrete time level

stage in a multistage RUNGE-KUTTA integration scheme

Subscripts

[]$_{0} \quad$ stagnation value

[ $]_{1}$ position of maximum tangential velocity

[]$_{1,2,3}$ cartesian component in $x, y, z$ direction

[]$_{1 / 2} \quad$ splitted value

[]$_{1 D}$ one-dimensional flows

[]$_{3 D}$ three-dimensional flows

[]$_{A}$ advective

[] $]_{A U S M D}$ splitting according to the $A U S M D$ scheme

[] $]_{A U S M V}$ splitting according to the $A U S M V$ scheme

[] $]_{A U S M D V}$ splitting according to the AUSMDV scheme

[]$_{b}$ boundary value 


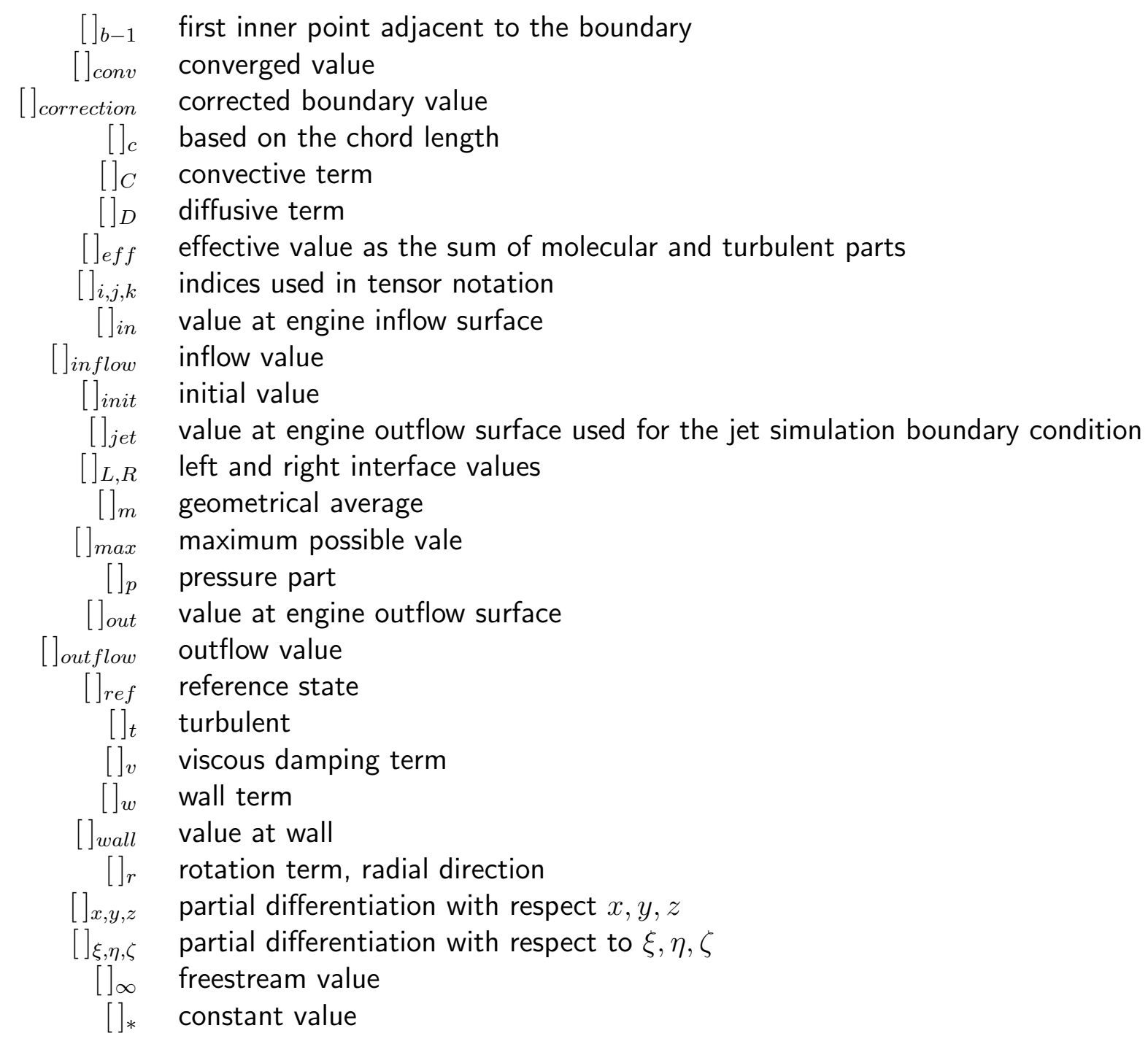

Acronyms

$A U S M \quad$ advective upstream splitting method

CFL COURANT-FrIEDRICHS-LEVy number

$D N S$ direct numerical simulation

$F A S$ full-approximation storage

$F D S$ flux difference splitting

$F V S$ flux vector splitting

$L E S$ large eddy simulation

$L O D I \quad$ local one-dimensional inviscid

$M P I$ message-passing interface

$M U S C L$ monotonic upstream centered scheme for conservation laws

$P D E$ partial differential equation

$R M S$ root mean square

$S A$ SPALART-ALLMARAS 


\section{Chapter 1}

\section{Introduction}

"After making an initial analysis of the flight voice and data recorders, US crash investigators believe the American Airlines airbus was hit twice by powerful wake turbulence from the plane immediately ahead...

In its final few seconds of flight the plane veered to the left and entered a nose-dive from 2,900 feet... All on board the plane were killed..."

BBC News on flight 587 crash November 12, 2001.

The increasing aircraft traffic requires optimized take-off and landing frequencies. On the one hand, the wake of airplanes represents a hazard to the following aircraft and was responsible for many fatal accidents in civil aviation. Consequently, the distance between airplanes especially during landing and take-off has to be enlarged to ensure safety. On the other hand, an increase in civil flight frequency and hence a decrease in distance between airplanes in the vicinity of airports means direct economical benefit to airports, airlines, aircraft manufacturers and passengers. Clearly, the wake hazard problem represents only one but an important aspect in the global development of aircraft traffic. An accurate prediction of the wake flow and a physical understanding of the complex behavior of the wake is a must for the optimization of the take-off and landing frequencies without a decline in safety.

The empirically stipulated minimum distance of landing or starting airplanes is usually based on the assumption that the circulation of the trailing vortices is determined mainly by the weight and hence the lift of the generating aircraft and that this circulation decreases by dissipative mechanisms in the downstream direction. Currently, the separation standards implemented worldwide by different air traffic control agencies [87] are determined mainly by weight categories of the leading and the following aircraft. Nevertheless, the BoEING 757 represents a known exception to the originally 
stipulated separation distances and hence demonstrates the intricacy of the wake flow problem.

In a recent review on the dynamics of trailing aircraft vortices by SPALART [112] different dissipative and convective mechanisms for the decay or collapse of the trailing vortices and environmental influences are discussed. The understanding and the exploitation of these mechanisms to enforce a premature destruction of the trailing vortices and consequently attenuate the wake hazard has been part of many academic and industrial research topics over the last 30 years. Unfortunately, none of the proposed constructive measures added to the generating aircraft up to date ever found technical application due to general performance losses. Neither was the scientific effort in understanding the wake flow over that period of research time capable of changing the air traffic control practices in stipulating the separation distances. An excellent overview of the history and the current status of research activities in this field supported by the Federal Aviation Administration FAA and the National Aeronautics and Space Administration NASA is presented in the work of Rossow [92].

The development of new very large airplanes like the AIRBus $A 380$ revived to some extent the enthusiasm again in this research field. One emphasis in current research topics is the investigation of instabilities of trailing vortices. Besides the classical long wave CROW instability [15] of a vortex pair attributed to mutual self-induced velocities of the vortices several other short wave instabilities of four vortex systems comparable to the vortex system of landing or starting airplanes [13, 23], the short wave WIDNALL instability [137] of a vortex in a weakly strained flow and the short wave instability of vortex pairs [55] were also observed. Especially the analysis done by CROUCH [13] describing new short wave instabilities with grow rates $60-100 \%$ greater than the CROW instability is considered to give the basis for a promising technical application to actively enforce the breakup of airplane trailing vortices [14]. However, measurements by HÜNECKE [43] of the wake of different AIRBUs models demonstrate from an industrial point of view that the interaction of the engine jet, flap side-edge and trailing edge vortices also can lead to a pronounced attenuation of the wake hazard depending on the spanwise position of the outboard engine and the jet exhaust shape. Another important aspect of the engine jet wake interaction, which is studied by QUACKENBUSH et al. [82] using simplified numerical methods, is the entrainment and detrainment of the engine exhaust into the vortex cores, which is relevant in the study of aircraft emissions in the atmosphere. Nevertheless, in spite of the technical importance of the interaction between the vortex system and the engine jet in the wake it is insufficiently understood and only few studies were dedicated to this topic until today.

One of the earliest works is by SCORER and DAvEnPORT [100] who investigated the instability of the condensation trails of airplanes at cruise altitudes. They attributed incorrectly the CROW instability, which was correctly explained later [15], to buoyancy effects resulting from the engine exhaust. JACQUIN and GARNIER [45] used simple integral methods to investigate the interaction of the engine jet and the vortex wake of a typical large transport aircraft under cruise conditions. They argued that the engine jet has almost no effect on the vortex wake dynamics up to 30 wingspans behind the airplane, which is in contrast to the aforementioned experimental results 
of HüNECKE [43]. This is not surprising, since their vortex wake model did not include axial or radial velocity components and therefore cannot predict the complex three-dimensional development of the wake vortices accurately. GERz and EHrET [32] concentrated in their numerical work using vortex-filament techniques followed by a time developing large eddy simulation of the wake on the entrainment/detrainment of engine exhausts into the vortex cores and did not investigate the influence of the engine jet on the development of the wingtip vortices. A similar numerical large eddy simulation of realistic aircraft wakes by LEWELLEN and LEWELLEN [56] included the engine jet and showed good agreement with observations of the CROw instabilities but did not cover a systematic investigation of particular parameters like the spacing between the engine and the wingtip and their influence on the dynamics of the trailing vortices. It is one of the main objectives of this work to investigate exactly such parameters and give details about their influence by numerically simulating the formation and the development of the wingtip vortex of a rectangular wing with variation of the engine jet parameters.

The formation and development of an isolated wingtip vortex was investigated experimentally and numerically in numerous works. Recent detailed experimental analysis of the structure and the wandering of the turbulent vortex during roll-up are e.g. the work of Devenport et al. [17] and CHOw et al. [11]. The latter experiment of a rectangular wing with small aspect ratio was also numerically simulated by DACLES-MARIANI et al. [16]. It was shown in the numerical investigation that good agreement with experimental results could only be achieved if appropriate grid resolution, turbulence models and boundary conditions were used. Computations of the vortex formation over a flap side-edge by KHORRAmi et al. [49] also corroborate the high computational demands to achieve a good agreement with measurements of the same configuration [83]. These experimental and numerical studies are limited in the near wake to the direct vicinity of the generating wing and did not extend the investigation to more than one chord length of the wing. Other separate calculations of the wake without including the generation process of the vortices can cover a domain from one to many wingspans depending on the numerical methods used and the accuracy achieved. A numerical simulation of the spatially developing wake of a high lift configuration up to 10 wingspans including all three-dimensional and turbulent effects was performed recently by SPALART et al. [115] using the REYNOLDS averaging concept and comparing three turbulence models. The calculations gave good results for the trajectories and structure of the vortices in the wake using a modified SPALART-AlLmARAS turbulence model but no quantitative validation of the proposed modifications to the turbulence model was presented and the magnitude of vorticity was generally underestimated partly due to the coarse resolution of the grid. It was pointed out in previous works $[25,26,27]$ that the resolution of the vortex cores is crucial to the accuracy of the wake calculations. The length scale of the vortex core is one or two magnitudes smaller than that of the generating wing. The necessary requirement of a sufficient resolution of the vortex cores and the shear layer of the wing wake increases considerably the amount of the grid points. However, a reduction of grid points can be achieved using higher-order discretization methods as will be shown in this work. 
It is commonly understood in the wake-vortex community that turbulence modeling of the wake especially within the vortex cores represents an ongoing research topic. Direct numerical simulations $D N S$ resolving all existent time and length scales as well as large eddy simulations $L E S$ resolving the time dependent large scales are currently limited in their application due to the large demand of computer resources. REYNOLDS averaging techniques on the other hand represent an applicable alternative for such complex flows of technical interest, but still have limitations in predicting accurate solutions for the wake with embedded vortices and the near-wall turbulence simultaneously. Therefore, a completely new turbulence model fulfilling this demand will be derived and validated in this work.

This thesis is a purely numerical study of the interaction of wingtip vortices and engine jets in the near field. Existing experiments are used whenever possible to validate the results and the numerical methods used. The work consists of two parts. The first part gives details about the numerical simulation techniques. Several newly developed methods like a new one-equation turbulence model, boundary conditions and discretization are derived, validated and discussed. Comparisons with other methods as well as experimental data are given to show the benefit of the proposed methods. The second part represents the discussion of the numerical simulation of the flow around the wing and finally in the wake. Relevant aspects of the flow and tendencies in the interaction of the engine jet and the wingtip vortices are shown for six configurations of a rectangular wing. The engine position in the wingspan direction and the engine jet exit velocity are considered to be the main two factors and are varied to investigate their effect. The geometry and the flow parameters are chosen according to experiments carried out separately as part of the collaborative research program SFB 401 at the RWTH AACHEN.

The organization of this text is as follows. Chapter 2 contains the mathematical description of the flow based on the NAVIER-STOKES equations. Turbulent effects are modeled according to the well established REYNOLDS averaging concept that is given in chapter 3. A discussion of the existing turbulence models especially one-equation models and their deficiencies is given. The well known Spalart-Allmaras model is presented and a derivation of a new model, which possesses several enhancements for the flow phenomena essential in jet-wingtip interaction flows, is given. Also a partial differential equation for the efficient calculation of the wall distance that is used in many turbulence models is introduced and its benefits are discussed.

Chapter 4 describes the details of the numerical scheme and its implementation. The discretization of the governing equations, the numerical boundary conditions as well as the acceleration techniques to steady state are documented. The objective of this chapter is to give a consistent and clear description of the methods to make a reproduction of the simulation results possible. Several details about the mathematical and numerical formulation are shifted into the appendices A-G to enable an easier reading and understanding of the essential ideas.

The first part of chapter 5 consists of validations and comparisons of the previously 
proposed methods especially the newly derived one-equation turbulence model. The second part discusses the flow around the rectangular wing in clean configuration with the engine jets and the wake flow. The focus is on the interaction of the wingtip vortex and the engine jet and the influence of the variation of the parameters engine position and jet velocity.

Finally, the conclusions are given in chapter 6, together with recommendations for future work. 


\section{Chapter 2}

\section{Mathematical Model}

"Mathematics up to the present day has been quite useless to us in regard to flying."

Annual Proceedings of the British Royal Aeronautical Society, 1879.

There are several physical theories that can be used to describe the motion of a fluid. At a fundamental microscopic level the particles of a fluid obey the theory of quantum mechanics and hence their dynamical evolution is described by the ScHRöDINGER equations. If quantum effects like interferences of wave packets can be neglected, which is known as the EHRENFEST's theorem [104], then the time evolution of the position and velocity coordinates of particles can be described by classical mechanics according to NEWTON's laws. A higher level of approximation doesn't include all the particles of a fluid independently but introduces a distribution function $\mathcal{F}(\vec{x}, \vec{u}, t)$ of the particle density at position $\vec{x}$ with the velocity $\vec{u}$ at time $t$. The time derivative of this distribution is determined by the Boltzmann equation. Finally, at the macroscopic level of approximation the fluid is considered to be a continuum lacking a molecular structure. This assumption is justified because the macroscopic length scales in most fluid dynamics problems are much larger than the molecular length scale of the fluid itself. A representation of the ratio between microscopic and macroscopic length scale is expressed through the KNUDSEN number $K n=l_{0} / L$, where $l_{0}$ represents the mean free path of the molecules and $L$ the characteristic macroscopic length scale. The order of the KNUDSEN number for turbulent flows can be approximated [122] by

$$
K n \approx \frac{3}{2} \frac{M a}{R e}
$$

where $M a$ is the MACH number and Re represents the REYNOLDS number. Typical values for turbulent flows are $M a=\mathcal{O}(1)$ and $R e=\mathcal{O}\left(10^{6}\right)$ which satisfies the assumption $K n \rightarrow 0$.

Experimental investigations of turbulent flows of liquids and gases, for example [118], 
Chapter 2. Mathematical Model

show excellent correlation. Even though both fluids have completely different molecular structure they still behave similarly under similar conditions, which proves that the macroscopic and microscopic behavior of fluids are independent.

According to the continuum hypothesis the equations of motion of a fluid can be derived by simplifying the BOLTZMANN equations through the CHAPMAN-ENSKOG series expansion [20] or directly from the basic conservation equations of mass, momentum and energy.

\subsection{The Navier-Stokes Equations}

The mathematical formulation of the hydrodynamic theory for an ideal fluid was first introduced by EuLER in 1755 [21]. Since the theory neglected the effects of viscosity it failed to give explanations to several phenomena that were observed in nature like vortex generation or viscous and pressure drag. Therefore, a new empirical science called hydraulics based on observations and measurements was founded and successfully used in technical applications like pipe flows. NAVIER [69] stated in 1822 the basic equations for an incompressible and viscous fluid, which were extended by POISSON [78] in 1831 to compressible flows. The coupling of the stress tensor and the deformation tensor were introduced by STOKES [120] in 1845, which was the final step in a complete and realistic mathematical model for the flow of a continuum.

The complete equation of motion, called the NAVIER-STOKES equations, describe the dynamics of an unsteady, viscous and compressible flow including heat transfer ${ }^{1}$. The flow dynamics of the conservative variables $\vec{Q}$

$$
\vec{Q}=(\rho, \rho \vec{v}, \rho e)^{T}
$$

representing the density $\rho$, the specific momentum $\rho \vec{v}$ and the specific energy $\rho e=$ $\rho\left(\epsilon+\frac{1}{2} \vec{v}^{*^{2}}\right)$, which includes the internal and the kinetic energy, is defined in the integral form of the equation

$$
\int_{\mathcal{V}} \frac{\partial \vec{Q}}{\partial t} d \mathcal{V}+\oint_{\mathcal{S}} \vec{F} \cdot \vec{n} d \mathcal{S}=0
$$

Equation 2.3 determines the temporal change of $\vec{Q}$ in a volume $\mathcal{V}$ surrounded by the surface $\mathcal{S}$ to be equivalent and of opposite sign to the total contribution from the flux $\vec{F}$ through $\mathcal{S}$ with the normal vector $\vec{n}$ pointing outwards. For continuous fluxes lacking contact discontinuities and shocks a differential form of the conservation laws can be derived using the Gauss theorem leading to

$$
\frac{\partial \vec{Q}}{\partial t}+\frac{\partial \vec{F}_{1}}{\partial x}+\frac{\partial \vec{F}_{2}}{\partial y}+\frac{\partial \vec{F}_{3}}{\partial z}=0
$$

where the subscripts $1,2,3$ denote the cartesian components of the flux $\vec{F}$ in the directions $x, y, z$. The flux $\vec{F}$ is subdivided into an advective part $\vec{F}_{A}$ containing convective

\footnotetext{
${ }^{1}$ volume forces such as gravitational forces are ignored here
} 
transport and pressure terms and a diffusive part $\vec{F}_{D}$ generated by friction and heat flow

$$
\vec{F}=\vec{F}_{A}-\vec{F}_{D}=\left(\begin{array}{c}
\rho \vec{v} \\
\rho \vec{v} \vec{v}+p \\
\rho \vec{v} e
\end{array}\right)-\left(\begin{array}{c}
0 \\
\overline{\bar{\tau}} \\
\overline{\bar{\tau}} \vec{v}+\vec{q}
\end{array}\right)
$$

with $\vec{q}$ denoting heat conduction given by the FOURIER law

$$
\vec{q}=-\lambda \nabla T
$$

where $T$ is the temperature and $\lambda$ is the thermal conductivity coefficient. The viscous shear stress tensor $\overline{\bar{\tau}}$ can be given for a NEWTONIAN fluid as

$$
\overline{\bar{\tau}}=\mu\left(\frac{\partial u_{i}}{\partial x_{j}}+\frac{\partial u_{j}}{\partial x_{i}}\right)-\frac{2}{3} \mu \delta_{i j} \frac{\partial u_{i}}{\partial x_{j}}
$$

where $\mu$ is the dynamic viscosity of the fluid. The assumption made by STOKES [120] that a second independent viscosity coefficient $\mu_{V}$ also called the volume viscosity can be neglected is experimentally validated and is adopted here. The viscosity coefficient $\mu$ of air is regarded to be a function of temperature according to an empirical power law for small temperature changes in relation to a reference state $($ ref $)$

$$
\mu=\mu_{\text {ref }}\left(\frac{T}{T_{\text {ref }}}\right)^{0.72} .
$$

Furthermore, it is known from measurements that the PRANDTL number for air is constant and has the value

$$
\operatorname{Pr}=\frac{\mu c_{p}}{\lambda}=0.72
$$

The specific heat coefficient at constant pressure $c_{p}$ is also constant for an ideal gas, which means that the thermal conductivity coefficient $\lambda$ follows the same power law as in equation (2.8)

$$
\lambda=\lambda_{\text {ref }}\left(\frac{T}{T_{\text {ref }}}\right)^{0.72} .
$$

Finally, the equation of state for an ideal gas

$$
p=\rho R T
$$

gives the relation between the thermodynamical variables $p, \rho$ and $T$, where $R=c_{p}-c_{v}$ is the gas constant per unit of mass. The specific internal energy $\epsilon$ and the specific enthalpy $h$ for calorically perfect gases can be given according to

$$
\epsilon=c_{v} T \quad h=c_{p} T
$$

The aforementioned set of equations is nondimensionalized using reference values at stagnation state shown in appendix A and are given explicitly in appendix C. 


\subsection{The Time-Averaged NAvier-Stokes Equations}

The classical approach to describe turbulent flows goes back to REYNOLDS in 1895, who introduced the concept of time averaging. The basic idea is to concentrate on the flow behavior on the relevant time scale and try to avoid resolving the unsteady turbulent flow fluctuations. A flow quantity $\Phi(\vec{x}, t)$ is separated into an averaged value $\bar{\Phi}(\vec{x}, t)$ and a fluctuating value $\Phi^{\prime}(\vec{x}, t)$

$$
\Phi(\vec{x}, t)=\bar{\Phi}(\vec{x}, t)+\Phi^{\prime}(\vec{x}, t) .
$$

The averaging is introduced with

$$
\bar{\Phi}(\vec{x}, t)=\frac{1}{\triangle t} \int_{t}^{t+\triangle t} \Phi(\vec{x}, t) d t
$$

where $\triangle t$ is to be chosen larger than the time scale of turbulence but still smaller than all time scales of the unsteady phenomena not associated with turbulence. If no such unsteady phenomena exist then the average quantity $\bar{\Phi}(\vec{x})$ will only be a function of space. Many turbulent flows like those presented in this work fall into this category, therefore the choice of $\Delta t$ will not be relevant and the possibility of overlapping time scales addressed in the spectral-gap-problem [139] is not present here.

The described time averaging can be applied to the NAVIER-STOKES equations which leads to equations for the averaged quantities and to correlations, which are products of fluctuation quantities that do not disappear after averaging. To minimize the number of correlations and to avoid density correlations a density-weighted average, called FAVRE average [66], $\tilde{\Phi}(\vec{x})$ is introduced

$$
\Phi(\vec{x}, t)=\tilde{\Phi}(\vec{x})+\Phi^{\prime \prime}(\vec{x}, t)
$$

with

$$
\tilde{\Phi}(\vec{x})=\frac{1}{\triangle t} \int_{t}^{t+\triangle t} \frac{\Phi(\vec{x}, t) \rho(\vec{x}, t)}{\bar{\rho}(\vec{x})} d t=\frac{\overline{\rho \Phi}}{\bar{\rho}} .
$$

Taking the REYNOLDS average of density $\rho$ and pressure $p$ and FAVRE average of the rest of the flow variables and applying the averaging rules presented in appendix $B$ leads to averaged equations similar to the instantaneous equations presented in section 2.1 with the addition of one term in the momentum equation and several other terms in the energy equation, a complete derivation of which is given in [139]. The term appearing in the momentum equation, called the FAVRE-averaged REYNOLDS-stress tensor,

$$
\overline{\bar{\tau}}_{t}=-\overline{\rho \vec{v}^{\prime \prime} \vec{v}^{\prime \prime}}
$$

is unknown and cannot be given explicitly without introducing new higher order correlations or some kind of modeling based on theoretical and empirical considerations. The FAVRE-averaged energy equation contains the terms

$$
\begin{aligned}
\bar{\rho} k & =\frac{1}{2} \overrightarrow{\rho \vec{v}^{\prime \prime} \cdot \vec{v}^{\prime \prime}} \\
\overrightarrow{q_{t}} & =\frac{\rho \vec{v}^{\prime \prime} e^{\prime \prime}}{}
\end{aligned}
$$


where $k$ represents the turbulent kinetic energy and $\overrightarrow{q_{t}}$ the turbulent heat transport. One further term $\overline{\bar{\tau}} \vec{v}^{\prime \prime}$ describes the molecular diffusion of turbulent kinetic energy, which is usually ignored for MACH numbers up to the supersonic regime [139]. The impossibility to express these correlations in terms of other averaged flow quantities without the introduction of new equations or other unknown correlations is known as the closure problem [106]. In chapter 3 turbulence models will be presented that close the system of equations and enable a complete mathematical description of turbulent flows.

\subsection{Initial and Boundary Conditions}

Several initial and boundary conditions are required to completely describe a specific flow problem. Some physical conditions are not known explicitly or may only be fulfilled at infinity, which is difficult to realize numerically.

\subsubsection{Initial Conditions}

For external flow problems an initially uniform flow at $t=t_{\text {init }}$ with the freestream values

$$
\vec{v}_{\text {init }}=\vec{v}_{\infty} \quad p_{\text {init }}=p_{\infty} \quad \rho_{\text {init }}=\rho_{\infty}
$$

is assumed. The boundary conditions are imposed for $t>t_{\text {init }}$, which corresponds to an instantaneous acceleration of a body in the domain from zero velocity to freestream velocity.

\subsubsection{Boundary Conditions at Solid Walls}

The boundary conditions at a solid non-moving adiabatic wall without mass transfer through the surface is given by

$$
\vec{v}_{\text {wall }}=\left.\overrightarrow{0} \quad \frac{\partial p}{\partial \vec{n}}\right|_{\text {wall }}=\left.0 \quad \frac{\partial \rho}{\partial \vec{n}}\right|_{\text {wall }}=0
$$

The condition for the pressure $\left.\frac{\partial p}{\partial \vec{n}}\right|_{\text {wall }}=0$ is a simplification taken from the boundary layer theory [106] and a more accurate value for the normal gradient $\left.\frac{\partial p}{\partial \vec{n}}\right|_{\text {wall }}$ can be derived by solving the momentum equation normal and tangential to the surface. This is usually not needed for shear layers at high REYNOLDS numbers [41] and without strong curvature effects, since then the boundary layer assumptions are fulfilled. The condition for density $\left.\frac{\partial \rho}{\partial \vec{n}}\right|_{\text {wall }}=0$ is derived from the adiabatic condition $\vec{q}_{\text {wall }}=0$ and the condition for pressure together with the relation for an ideal gas (2.11). 


\subsubsection{Boundary Conditions at Inflow/Outflow}

According to the analysis of Gustafsson and Sundström [38] the NAVIER-Stokes equations require five boundary conditions to be imposed at the inflow boundary and four conditions at the outflow boundary. As noted in [41] care should be taken in imposing the boundary conditions, since the EulER equations have different requirements. Consistent conditions for the viscous flow have to be compatible with the inviscid flow at the limit of vanishing viscosity. The discussion of the formulation and the derivation of numerical inflow/outflow boundary conditions is given in chapter 4.5. 


\section{Chapter 3}

\section{Turbulence Modeling}

"Turbulence studies are the art of understanding the Navier-Stokes equations without actually solving them."

P. Bradshaw.

The time-averaged equations presented in chapter 2.2 are not closed, since due to the turbulent correlations there are less equations than unknowns. A turbulence model relates these unknowns to the mean flow variables and closes the system of equations thereby avoiding the time consuming exact spatial and temporal resolution of the flow, e.g. through direct numerical simulation $D N S$.

The first attempt to model the missing information goes back to BoussinesQ [6] in 1877 where he first introduced the concept of eddy viscosity. The BoussinesQ hypothesis is based on a formal similarity between the viscous stress tensor $\overline{\bar{\tau}}$ and the REYNOLDS stress tensor $\overline{\bar{\tau}}_{t}$, which assumes the principal axes of both tensors to be coincident with those of the main strain tensor $\overline{\bar{S}}$ for incompressible flows. In analogy to the STOKEs postulate for the viscous stress tensor in a laminar flow the relationship

$$
\overline{\bar{\tau}}_{t}=-\overline{\rho \vec{v}^{\prime \prime} \vec{v}^{\prime \prime}}=2 \mu_{t} \overline{\bar{S}}=\mu_{t}\left(\frac{\partial u_{i}}{\partial x_{j}}+\frac{\partial u_{j}}{\partial x_{i}}\right) \quad \text { for incompressible flows }
$$

with the proportionality coefficient $\mu_{t}$ is assumed. Unlike the molecular viscosity $\mu$ the value $\mu_{t}$ is not a fluid property but depends on many factors in the flow and is problem dependent. Similarly the turbulent heat flux $\vec{q}_{t}$ is modeled following the classical analogy between momentum and heat transfer [86], which assumes that it is proportional to the temperature gradient

$$
\vec{q}_{t}=\overline{\rho \vec{v}^{\prime \prime} e^{\prime \prime}}=-\frac{\mu_{t} c_{p}}{P r_{t}} \nabla T
$$

with a constant turbulent PRANDTL number $P r_{t}=0.9$ [106].

The assumption made by BOUssinEsQ is questioned by researchers (see discussion 
in [139]) and proven to be inaccurate for some flow problems like flows with separation and secondary motions. Nevertheless, many practical aerodynamic flow problems are well and efficiently predicted using turbulence models based on this hypothesis.

Furthermore, it is assumed that the influence of compressibility, e.g. density fluctuations, can be neglected for MACH numbers below 5, which is known as the MorkOvIN hypothesis [66]. This implies that the analysis for turbulent incompressible flows using turbulence models based on the incompressible flow equation remains also valid for compressible flows within the MACH number limit. The REYNOLDS stress tensor $\overline{\bar{\tau}}_{t}$ can then be written for compressible flows according to similarity to the viscous counterpart

$$
\overline{\bar{\tau}}_{t}=\mu_{t}\left(\frac{\partial u_{i}}{\partial x_{j}}+\frac{\partial u_{j}}{\partial x_{i}}\right)-\frac{2}{3} \mu_{t} \delta_{i j} \frac{\partial u_{i}}{\partial x_{j}}
$$

which introduces only a minor change in the formulation that is important only at high MACH numbers. Replacing the expressions for $\overline{\bar{\tau}}_{t}$ and $\vec{q}_{t}$ in the averaged NAVIERSTOKES equations leads to equations formally identical to the unaveraged equations (2.4) with new effective values for $\mu$ and $\lambda$

$$
\mu_{e f f}=\mu+\mu_{t} \quad \text { and } \quad \lambda_{e f f}=-\frac{\mu c_{p}}{P r}+\frac{\mu_{t} c_{p}}{P r_{t}}
$$

The closure problem described earlier reduces thereafter to the modeling of $\mu_{t}$ or $\nu_{t}=\mu_{t} / \rho$ which is also referred to as eddy viscosity. Many models were introduced ranging from simple and incomplete algebraic models like the mixing length hypothesis by PRANDTL [76] to complete stress-transport models like the LAUNDER-REECERoDi Model [51]. However, the increasing complexity of the turbulence models and the larger computational effort when they are applied to realistic technical problems is not always justified by a qualitative improvement in the solutions. An overview on some of the standard and widely used turbulence models is given in [139].

In the following section the discussion will be concentrated on one-equation turbulence models. After an overview on the historical development of such models the popular Spalart-Allmaras model will be presented followed by a proposal for a new oneequation model. Finally a partial differential equation is derived for the wall distance $d$ used in many turbulence models. 


\subsection{One-Equation Turbulence Models}

One-equation turbulence models enjoyed a wide popularity in the last decade. The growing interest in this type of models is explained primarily by the numerical ease of use compared to standard two-equation models like the $k-\varepsilon$ [48] and $k-\omega$ models [138] that are still widely used. Algebraic models like the customary BALDWIN-LOMAX model [1] are efficient from a numerical point of view but lack generality and have deficits like the missing transport and diffusion effects. Therefore, one-equation models are considered to be a good compromise between the algebraic models and the classical two-equation models.

The first one-equation model for the turbulent kinetic energy $k$ was postulated by PRANDTL [77] and independently introduced by Emmons [19]. Both formulations were incomplete since they needed the definition of an application dependent length scale. Later Bradshaw, FERRIS and ATwell [8] proposed another model for $k$, based on the assumption that the REYNOLDS shear stress in a two-dimensional flow $\tau_{t_{x y}}$ is proportional to the turbulent kinetic energy $k$

$$
\tau_{t_{x y}}=\beta_{b} k \quad \text { with } \quad \beta_{b}=0.3
$$

which is called the Bradshaw hypothesis and was validated by Townsend [125] through various measurements in boundary layers, wakes and mixing layers.

The first formulation for a one-equation model directly for $\nu_{t}$ was postulated by NEE and Kovasznay [70] based on phenomenological arguments. A complete model for $\nu_{t}$ was also proposed by SECUNDOV [101] as an evolution of the former NEE and KoVASZNAY model with considerable success. Its current formulation is known as the $\nu_{t_{92}}$ version [109]. Unfortunately it was unknown in the English speaking community until its publication in 1995. In 1991 BALDWIN and BARTH [2] derived an elaborate transport model from the classical $k-\varepsilon$ model, which had limited success due to its inconsistent numerical behavior at turbulent/non-turbulent interfaces [64, 139]. The most successful one-equation model up to date was introduced in 1992 by SPALART and Allmaras [110] which was created generically for the turbulent eddy viscosity $\nu_{t}$ with strong emphasis on the numerical behavior. Later MENTER [64] proposed a general methodology for deriving one-equation models from two-equation models and suggested a new model based on the standard $k-\varepsilon$ equations, which shows superior results when compared to the BALdwin-BARTH model. NAgAno, Pei and HATTORI [68] also derived another one-equation model from the low-REYNOLDS-number $k-\varepsilon$ model with many closure and damping functions and showed good results compared with DNS and experiments for wall bounded flows like boundary layers and plane wall jets but did not investigate any aerodynamic or free shear flows.

The performance and the predictive capabilities of many models including the BALDWINLomax, Baldwin-BARTh, Spalart-Allmaras, standard $k-\varepsilon, k-\omega$ and the $S S T$ [63] models, are documented in many publications [3, 33, 90, 93, 99, 109, 134, 139] and corroborate the quality of the one-equation models, especially the SPALART-ALLMARAS model. This model is discussed in detail in the following section. Its original formulation and several modification that were published subsequently are presented. 


\subsubsection{The Spalart-Allmaras Model}

The SpalarT-Allmaras one-equation model [110] proved to be very efficient and robust for various challenging aerodynamic flows and was already successfully applied to calculations $[25,26,27,49]$ similar to those presented in this work. The model calculates the kinematic eddy viscosity

$$
\nu_{t}=\check{\nu} f_{v 1}
$$

via the following transport equation for $\check{\nu}$

$$
\frac{D \check{\nu}}{D t}=\underbrace{c_{b 1} \check{P} \check{\nu}}_{\text {production }}-\underbrace{c_{w 1} f_{w}\left(\frac{\check{\nu}}{d}\right)^{2}}_{\text {destruction }}+\underbrace{\frac{1}{\sigma}\left[\nabla \cdot((\nu+\check{\nu}) \nabla \check{\nu})+c_{b 2}(\nabla \check{\nu})^{2}\right]}_{\text {diffusion }} .
$$

The closure functions and coefficients are given as

$$
\begin{array}{rlrl}
f_{v 1} & =\frac{\chi^{3}}{\chi^{3}+c_{v 1}^{3}} & f_{v 2}=1-\frac{\chi}{1+\chi f_{v 1}} & \chi=\frac{\check{\nu}}{\nu} \\
f_{w}=g\left[\frac{1+c_{w 3}^{6}}{g^{6}+c_{w 3}^{6}}\right]^{\frac{1}{6}} & g=r+c_{w 2}\left(r^{6}-r\right) & r=\frac{\check{\nu}}{\check{P} \kappa^{2} d^{2}} \\
c_{b 1}=c_{b 1}=.1355 & c_{b 2}=0.622 & c_{v 1}=7.1 & \sigma=2 / 3 \\
c_{w 1}=\frac{1+c b 2}{\sigma}+\frac{c_{b 1}}{\kappa^{2}} & c_{w 2}=0.3 & c_{w 3}=2 & \kappa=0.41
\end{array}
$$

The production term $\check{P}$ was suggested to be primarily the norm of the rotation tensor $|\overline{\bar{\Omega}}|=1 / 2\left|\left(\nabla \vec{v}-(\nabla \vec{v})^{T}\right)\right|$ or the magnitude of vorticity $|\nabla \times \vec{v}|$

$$
\check{P}=P+\frac{\check{\nu}}{\kappa^{2} d^{2}} f_{v 2} \quad \text { with } \quad P=|2 \overline{\bar{\Omega}}|=|\nabla \times \vec{v}|
$$

The initial and boundary conditions for $\check{\nu}$ are

$$
\begin{aligned}
\check{\nu}_{\text {init }} & \approx 0.1 \nu & \check{\nu}_{\text {wall }} & =0 \\
\check{\nu}_{\text {inflow }} & =\nu_{\text {init }} & \left.\frac{\partial \check{\nu}}{\partial \vec{n}}\right|_{\text {outflow }} & =0 .
\end{aligned}
$$

The initial value of $\check{\nu}_{\text {init }} \approx 0.1 \nu$ was chosen higher than the suggested zero value [110] to avoid the trivial solution $\check{\nu} \equiv 0$ of equation (3.7) and to speed up the calculations by exciting the production term $P$ more rapidly.

The model does not include the turbulent kinetic energy $k$, which is simply dropped from the averaged equations [110]. This missing feature does not seem to play a decisive role in the computations presented in the original paper.

Since the transition tripping functions introduced by SPALART and Allmaras in the original work [110] are rarely implemented by researchers [139] due to numerical and implementation difficulties they will not be discussed here. 
There has been discussions [113] concerning the production and the destruction terms. The dependence of the production term on the vorticity is motivated by the fact that the near-wall vorticity is responsible for the generation of turbulence. However, this is not true in a vortex core, where rotation effects diminish turbulence, resulting in a decay of the overall turbulence within the vortex $[11,17,74]$. In $[27,16]$ it is therefore suggested to limit $P$ by the strain rate tensor $|\overline{\bar{S}}|$

$$
P=2(|\overline{\bar{\Omega}}|+\min (0,|\overline{\bar{S}}|-|\overline{\bar{\Omega}}|))
$$

Note that this modification has no influence on the near-wall region, where $|\overline{\bar{\Omega}}|$ and $|\overline{\bar{S}}|$ are of the same order of magnitude. Another modification to account for streamline curvature and rotation effects was introduced by SpalarT and SHuR [111] through the definition of a new function $f_{r 1}$

using

$$
f_{r 1}=\left(1+c_{r 1}\right) \frac{2 r^{*}}{1+r^{*}}\left(1-c_{r 3} \tan ^{-1}\left(c_{r 2} r^{*}\right)\right)-c_{r 1}
$$

$$
r^{*}=|\overline{\bar{S}}| /|\overline{\bar{\Omega}}| \quad \text { and } \quad c_{r 1}=1 \quad c_{r 2}=12 \quad c_{r 3}=1
$$

which is used to scale the production term $P$.

Furthermore, it could be considered a drawback of the model that the destruction term disappears completely in the far-wall region $(d \gg 1)$, which is not true for turbulent free shear flows, e.g. jets or wakes. The absence of this term leads to an unphysical growth of $\check{\nu}$ when a jet flow occurs far from a wall and, consequently, results in an over-prediction of the spreading rate as reported in [3, 139].

Modifications of the viscous damping $f_{v 1}$ and destruction term as well as transition modeling and adaptation to unsteady calculations have been suggested by EuLITz [22] with the objective of an accurate numerical simulation of the unsteady turbulent flow in turbo machinery. Free shear flows and vortical flows that are unsatisfactory predicted by the SPALART-Allmaras model were not investigated in his study.

The Spalart-Allmaras model is successful in numerous flow applications and some modifications were introduced to improve the model further as described formerly. Nevertheless, there is still potential for improvements especially for free shear flows. To achieve that a new one-equation turbulence model similar to the SPALART-ALLMARAS model is proposed in the next section.

\subsubsection{A New One-Equation Turbulence Model}

As mentioned in the last section the SPALART-AlLmaras model has deficiencies in regions far away from the wall and in free shear and vortical flows. The intention behind the new model is to introduce a complete and general one-equation model capable of correctly predicting a wide class of fundamental flows like boundary layer, wake, jet and vortical flows. Since these types of flows are all present in the simulations carried out in this work the quality of the overall solution is determined by the capability to capture the detailed physics of these flows. 
The starting point will be the mature and validated two-equation $k-\omega$ turbulence model of WILCOX [139] given in tensor notation

$$
\begin{aligned}
\frac{D k}{D t} & =\tau_{t_{i j}} \frac{\partial u_{i}}{\partial x_{j}}-\beta^{*} k \omega+\frac{\partial}{\partial x_{j}}\left[\left(\nu+\sigma^{*} \nu_{t}\right) \frac{\partial k}{\partial x_{j}}\right] \\
\frac{D \omega}{D t} & =\alpha \frac{\omega}{k} \tau_{t_{i j}} \frac{\partial u_{i}}{\partial x_{j}}-\beta \omega^{2}+\frac{\partial}{\partial x_{j}}\left[\left(\nu+\sigma \nu_{t}\right) \frac{\partial \omega}{\partial x_{j}}\right]
\end{aligned}
$$

with the following closure coefficients

$$
\sigma^{*}=\sigma=0.5 \quad \alpha=0.52 \quad \beta_{c}^{*}=0.09 \quad \beta_{c}=0.072
$$

and the closure functions

$$
\begin{array}{lll}
\beta^{*}=\beta_{c}^{*} f_{\beta}^{*} & f_{\beta}^{*}=\frac{1+680 \psi_{k}^{2}}{1+400 \psi_{k}^{2}} \quad \psi_{k}=\max \left(0 ., \frac{1}{\omega^{3}} \frac{\partial k}{\partial x_{j}} \frac{\partial \omega}{\partial x_{j}}\right) \\
\beta=\beta_{c} f_{\beta} & f_{\beta}=\frac{1+70 \psi_{\omega}}{1+80 \psi_{\omega}} & \psi_{\omega}=\left|\frac{\Omega_{i j} \Omega_{j k} S_{k i}}{\left(\beta_{c}^{*} \omega\right)^{3}}\right|
\end{array}
$$

using the definitions

$$
\begin{array}{cc}
\Omega_{i j}=\frac{1}{2}\left(\frac{\partial u_{i}}{\partial x_{j}}-\frac{\partial u_{j}}{\partial x_{i}}\right) & S_{i j}=\frac{1}{2}\left(\frac{\partial u_{i}}{\partial x_{j}}+\frac{\partial u_{j}}{\partial x_{i}}\right) \\
\nu_{t}=\frac{k}{w} & \tau_{t_{i j}}=2 \nu_{t} S_{i j}-\frac{2}{3} k \delta_{i j}
\end{array} .
$$

The functions $\psi_{k}$ and $\psi_{\omega}$ were added to the original model [138] to account for crossdiffusion and vortex-stretching, respectively [139]. They mainly improve the results for wake and jet flows without interfering with the good results achieved with the original model for boundary layer flows. Note that the $k-\omega$ model is one of the few turbulence models that do not need any viscous damping or have any explicit wall distance dependence in the equations. However, it possesses numerical difficulties in the vicinity of the wall, since the boundary condition $\omega_{\text {wall }} \rightarrow \infty$ produces high gradients near the wall and requires a higher resolution in the boundary layer. Further criticism [62, 63] concerning the freestream value $\omega_{\text {init }}$ and $\omega_{\text {inflow }}$ sensitivity are partly remedied in the new formulation of the $k-\omega$ model [139]. It should be noted that such criticism is neglecting the fact that the boundary values of a turbulence model like in any other boundary value problem should affect the solution, e.g., in the order of the freestream value, a fact that is rarely mentioned in the debate.

A PDE for $\nu_{t}$ can be derived using the definition of the eddy viscosity $k=\nu_{t} \omega$ and the total derivatives $D k / D t$ and $D \omega / D t$ similar to $[2,64]$ :

$$
\begin{aligned}
\frac{D \nu_{t}}{D t} & =\frac{1}{\omega}\left(\frac{D k}{D t}-\nu_{t} \frac{D \omega}{D t}\right) \\
& =(1-\alpha) \frac{\tau_{t_{i j}}}{\omega} \frac{\partial u_{i}}{\partial x_{j}}-\beta^{*} k+\beta \nu_{t} \omega \\
& +\frac{1}{\omega} \frac{\partial}{\partial x_{j}}\left[\left(\nu+\sigma^{*} \nu_{t}\right) \frac{\partial k}{\partial x_{j}}\right]-\frac{\nu_{t}}{\omega} \frac{\partial}{\partial x_{j}}\left[\left(\nu+\sigma \nu_{t}\right) \frac{\partial \omega}{\partial x_{j}}\right]
\end{aligned}
$$


The terms on the right-hand side can be rewritten ${ }^{1}$ by inserting $k=\nu_{t} \omega$ and using the equivalence $\sigma^{*}=\sigma$. Especially the diffusion terms on the right-hand side can be reformulated equivalently leading to the desired $P D E$ for $\nu_{t}$

$$
\begin{aligned}
\frac{D \nu_{t}}{D t} & =2(1-\alpha) \frac{\nu_{t} S_{i j}}{\omega} \frac{\partial u_{i}}{\partial x_{j}}-\frac{2}{3}(1-\alpha) \nu_{t} \delta_{i j} \frac{\partial u_{i}}{\partial x_{j}}-\left(\beta^{*}-\beta\right) \nu_{t} \omega \\
& +\frac{\partial}{\partial x_{j}}\left[\left(\nu+\sigma \nu_{t}\right) \frac{\partial \nu_{t}}{\partial x_{j}}\right]+2 \frac{\left(\nu+\sigma \nu_{t}\right)}{\omega} \frac{\partial \nu_{t}}{\partial x_{j}} \frac{\partial \omega}{\partial x_{j}}
\end{aligned}
$$

with a reformulation of $\psi_{k}$ defined previously in equation (3.17)

$$
\psi_{k}=\max \left[0 ., \frac{1}{\omega^{3}}\left(\nu_{t}\left(\frac{\partial \omega}{\partial x_{j}}\right)^{2}+\omega \frac{\partial \nu_{t}}{\partial x_{j}} \frac{\partial \omega}{\partial x_{j}}\right)\right] .
$$

It should be noted that since up to this point only a reformulation without any simplification has been carried out there is no loss in generality. The devision by $\omega$ in two terms on the right-hand side is numerically not a problem if $\omega \rightarrow 0$ since this is physically only possible in laminar flows $\nu_{t}=0$, which means that the numerator also tends to zero. Usually a $\max (\omega$, eps) with a small numerical value eps, e.g. the machine accuracy, is used to numerically prevent division by zero.

The value $\omega$ should be reconstructed explicitly based on flow quantities such as velocity gradients. By defining this reconstruction we can close our proposed equation. It is clear that this is the most essential step, since the generality of the reconstructed $\omega$ must be guaranteed throughout a wide range of flows.

The most famous assumption concerning such a reconstruction is the BRADsHAw hypothesis given in equation (3.5). This assumption is directly implemented into many turbulence models like in $[8,64]$ and is indirectly included in several other turbulence models like $k-\varepsilon[48]^{2}$ and $k-\omega$ [139]. For instance, by neglecting the convective, destructive, and dissipative terms of the turbulence energy equation (3.14) the aforementioned hypothesis can be easily derived

$$
\beta^{*} k \omega=\beta_{c}^{*} k^{2} / \nu_{t}=\tau_{t_{i j}} \frac{\partial u_{i}}{\partial x_{j}} \Rightarrow k=\frac{1}{\sqrt{\beta_{c}^{*}}} \sqrt{\nu_{t} \tau_{t_{i j}} \frac{\partial u_{i}}{\partial x_{j}}}
$$

and for $2 \mathrm{D}$ shear layers

$$
k=\frac{1}{\sqrt{\beta_{c}^{*}}} \tau_{t_{x y}}=\frac{\nu_{t}}{\sqrt{\beta_{c}^{*}}}\left|\frac{d u}{d y}\right| .
$$

In spite of its widespread use this hypothesis is neither exactly valid in the viscous sublayer of the turbulent boundary layer [68] nor in free shear layers [106], which was indicated by $D N S$ and experimental data. Several attempts based on dimensional analysis and on simplifications of the $k-\omega$ equations were studied to achieve other reconstructions. However, they usually lead to ill numerical behavior since they include

\footnotetext{
${ }^{1}$ Here we chose to eliminate $k$ from the equations. Other choices are also possible but more complicated and therefore not described here.

${ }^{2}$ Note the relation $C_{\mu}=\beta_{c}^{*}=\beta_{b}^{2}=0.09$ with $C_{\mu}$ being a closure coefficient of the $k$ - $\varepsilon$ model.
} 
higher order derivatives and cross derivatives of $u$ and $\nu_{t}$ as well as very high gradients near the wall, similar to the underlying $k-\omega$ model. On the one hand an exact reconstruction means an infinite value of $\omega_{\text {wall }} \rightarrow \infty$ at the wall, which on the other hand should be avoided for the sake of efficiency and stability of the numerical scheme. Consequently, a slight modification of the equation (3.21) and its closure functions $f_{\beta^{*}}, f_{\beta}$ are preferred in the formulation of the new one-equation turbulence model. The quantity $\omega$ is reconstructed according to the BRADSHAW hypothesis for $2 \mathrm{D}$ shear layers

$$
\omega=\frac{k}{\nu_{t}}=\frac{1}{\sqrt{\beta_{c}^{*}}}\left|\frac{d u}{d y}\right|
$$

and generalized using the norm of the strain tensor $S_{i j}[68]$

$$
\omega=\frac{1}{\sqrt{\beta_{c}^{*}}} \sqrt{2 S_{i j} S_{i j}} .
$$

In the next sections several tuning and addition of closure coefficients and closure functions is presented which were evaluated during the investigation of some flow categories like free shear and wall boundary layer flows.

\section{Free Shear Flows}

The calibration of the closure functions $f_{\beta^{*}}, f_{\beta}$ for wake and jet flows leads to the following new formulations

$$
f_{\beta}^{*}=\frac{1+1610 \psi_{k}}{1+1195 \psi_{k}} \quad \text { and } \quad f_{\beta}=\frac{1+64 \psi_{\omega}}{1+80 \psi_{\omega}} .
$$

WiLCOX introduced these functions and calibrated them to improve the original $k-\omega$ model for the same free shear flows. These functions do not play a role in the vicinity of the wall [139] since $\psi_{k}$ and $\psi_{\omega}$ approach zero near solid surfaces.

\section{Near-Wall Behavior}

The BRADSHAw hypothesis is exactly fulfilled in the logarithmic region of the boundary layer. This probably explains the success of this assumption in the turbulence modeling community. However, it is not valid in the viscous sublayer, where the laminar viscosity is dominating. Almost all turbulence models include damping terms near the wall to correct this behavior. Many of these terms are functions of the wall normal distance $d$. To avoid such a dependence the viscous damping function $f_{v 1}$ of MeLLOR and HERRING [61] similar to the approach applied in the SpalarT-Allmaras model was chosen

$$
\nu_{t}=\check{\nu} f_{v 1} \quad \text { with } \quad f_{v 1}=\frac{\chi^{3}}{\chi^{3}+c_{v 1}^{3}} \quad \text { and } \quad \chi=\frac{\check{\nu}}{\nu} .
$$

The coefficient $c_{v 1}=7.1$ was chosen according to the original value of MELLOR and HERRING. This approach allows $\check{\nu}$ to be equal to $\kappa u_{\tau} d$ in the logarithmic region and in 
the viscous sublayer. This behavior is considered one of the reasons for the success of the SPALART-AlLmaras model, since it enforces a numerically advantageous linear growth of $\check{\nu}$ in the vicinity of the wall. Recall that $f_{v 1}$ is important only in the viscous region, where $\chi$ is of $\mathcal{O}(1)$, while its influence disappears in the logarithmic region. For free shear flows it has a limited impact at turbulent/non-turbulent edges, where $\chi$ is also of $\mathcal{O}(1)$. In these regions we expect the turbulent eddy viscosity $\nu_{t}$ to be of small influence and therefore not to interfere with our former calibration for the free shear flows.

There are investigations of the correct wall limiting behavior of $\nu_{t_{d \rightarrow 0}}[68,139]$ that will not be discussed here, since the laminar viscosity $\nu$ in the viscous sublayer is the dominating quantity. Hence, good results can be expected for wall bounded flows even if $\nu_{t}$ is not asymptotically consistent at the wall.

\section{Decay of Isotropic Turbulence}

Experimental investigation [125] indicate that the turbulent kinetic energy $k$ of an isotropic homogeneous turbulent flow should decay according to

$$
k(t) \sim t^{-n} \quad \text { with } \quad n=1.25 \pm 0.06
$$

The $k-\omega$ model can be simplified by dropping all the spatial gradients

$$
\frac{d k}{d t}=-\beta_{c}^{*} \omega k \quad \text { and } \quad \frac{d \omega}{d t}=-\beta_{c} \omega^{2}
$$

and solved analytically for large $t$

$$
k(t) \sim t^{-\beta_{c}^{*} / \beta_{c}}=t^{-5 / 4} \quad \text { and } \quad \omega(t) \sim \frac{1}{\beta_{c} t}
$$

which is in good agreement with experiments. Similar to other one-equation models the proposed one-equation model in equation (3.21) does not predict a decay at all, since all terms include spatial derivatives that vanish in a homogeneous flow leading to $\frac{d \nu_{t}}{d t} \equiv 0$. The correct decay of $\nu_{t}$ can be derived from the $k-\omega$ equation

$$
\nu_{t}(t)=k(t) / \omega(t) \quad \Rightarrow \quad \nu_{t}(t) \sim t^{1-\beta_{c}^{*} / \beta_{c}}=t^{-1 / 4} .
$$

To achieve this behavior a generic term is added to equation (3.21)

$$
\frac{d \nu_{t}}{d t}=-k_{i n i t}\left(\phi \frac{\nu_{t}}{\nu}\right)^{\frac{-\beta_{c}^{*} / \beta_{c}}{1-\beta_{c}^{*} / \beta_{c}}}=-k_{\text {init }}\left(\phi \frac{\nu_{t}}{\nu_{\text {init }}}\right)^{5}
$$

where $k_{\text {init }}$ represents the initial turbulent kinetic energy that can be calculated from the initial, i.e. prescribed, turbulence intensity $T u$

$$
T u=\sqrt{\frac{u^{\prime \prime} u^{\prime \prime}+v^{\prime \prime} v^{\prime \prime}+w^{\prime \prime} w^{\prime \prime}}{3 u_{\infty}^{2}}}=\sqrt{\frac{2 k}{3 u_{\infty}^{2}}} .
$$


Furthermore, the following relation can be derived by using equations (3.30) for $t=t_{\text {init }}$

$$
\left.\frac{d \nu_{t}}{d t}\right|_{\text {init }}=\left.\frac{1}{\omega}\left(\frac{d k}{d t}-\frac{k}{\omega} \frac{d \omega}{d t}\right)\right|_{\text {init }}=\left(\beta_{c}-\beta_{c}^{*}\right) k_{\text {init }}=-k_{\text {init }}\left(\phi \frac{\nu_{t}}{\nu_{\text {init }}}\right)^{5} \text {. }
$$

This leads to the value of $\phi=-\left(\beta_{c}-\beta_{c}^{*}\right)^{1 / 5} \approx 0.4478$. The small value of $\phi<1$ taken to the power of 5 does not interfere with the former calibration of the model.

A simulation of isotropic turbulence using the original $k-\omega$ model and the new model with the additional term given in equation (3.33) is presented in figure 3.1 showing the excellent agreement between the proposed term and the predicted decay of the original model.

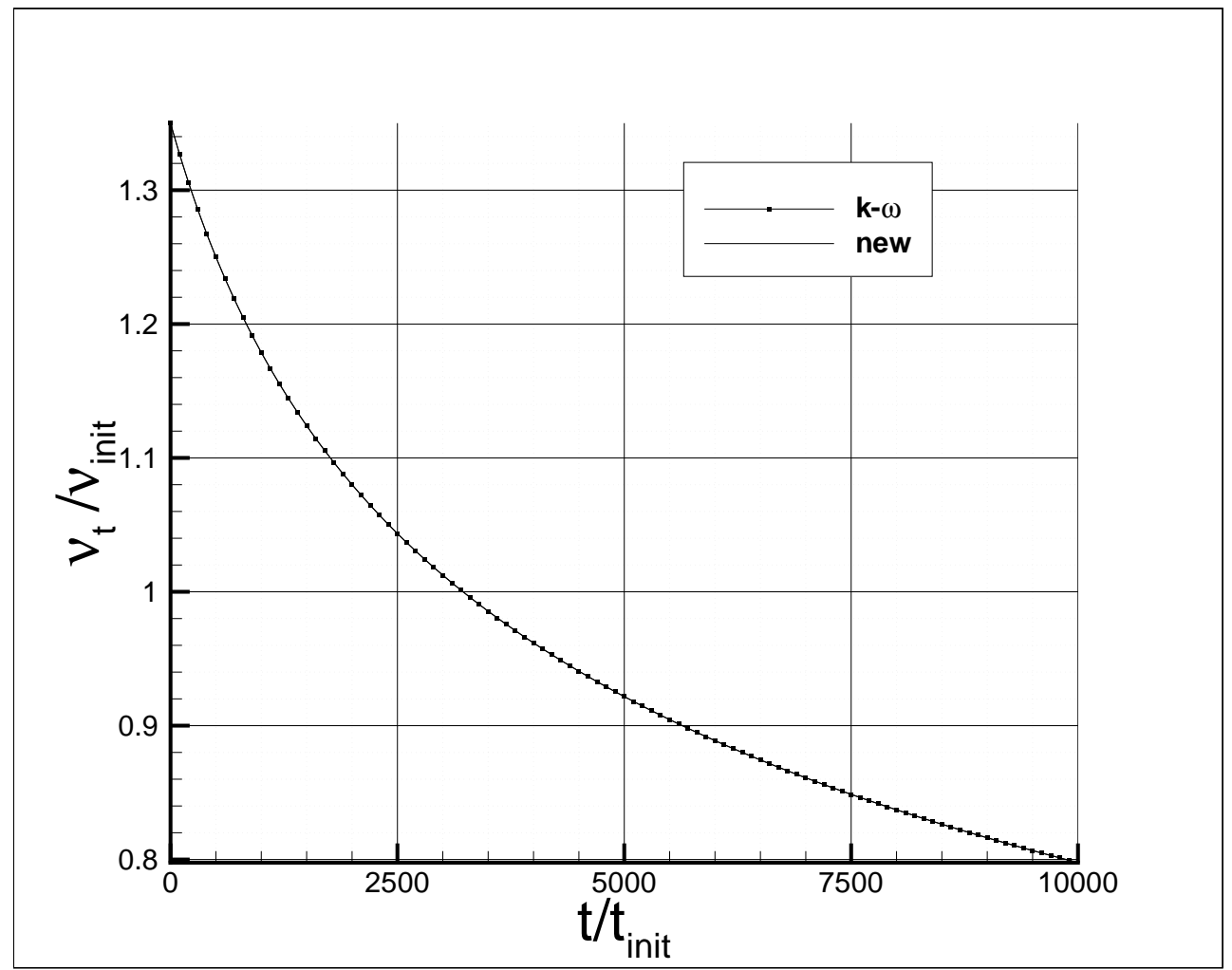

Figure 3.1: Decay of isotropic turbulence

\section{The Final Version of the Model}

Using

$$
\nu_{t}=\check{\nu} f_{v 1} \quad \text { with } \quad f_{v 1}=\frac{\chi^{3}}{\chi^{3}+c_{v 1}^{3}} \quad \text { and } \quad \chi=\frac{\check{\nu}}{\nu}
$$

the new one-equation turbulence model reads for the dependent variable $\check{\nu}$

$$
\begin{aligned}
\frac{D \check{\nu}}{D t} & =2(1-\alpha) \frac{\check{\nu}}{\omega} S_{i j} \frac{\partial u_{i}}{\partial x_{j}}-\frac{2}{3}(1-\alpha) \check{\nu} \delta_{i j} \frac{\partial u_{i}}{\partial x_{j}}-\left(\beta^{*}-\beta\right) \check{\nu} \omega-k_{\text {init }}\left(\phi \frac{\check{\nu}}{\nu_{\text {init }}}\right)^{5} \\
& +\frac{\partial}{\partial x_{j}}\left[(\nu+\sigma \check{\nu}) \frac{\partial \check{\nu}}{\partial x_{j}}\right]+2 \frac{(\nu+\sigma \check{\nu})}{\omega} \frac{\partial \check{\nu}}{\partial x_{j}} \frac{\partial \omega}{\partial x_{j}}
\end{aligned}
$$


with the closure coefficients

$$
\sigma=0.5 \quad \alpha=0.52 \quad \beta_{c}^{*}=0.09 \quad \beta_{c}=0.072 \quad c_{v 1}=7.1 \quad \phi=\left(\beta_{c}-\beta_{c}^{*}\right)^{1 / 5}
$$

and the closure functions

$$
\begin{aligned}
& \beta^{*}=\beta_{c}^{*} f_{\beta}^{*} \quad f_{\beta}^{*}=\frac{1+1610 \psi_{k}}{1+1195 \psi_{k}} \quad \psi_{k}=\max \left[0 ., \frac{1}{\omega^{3}}\left(\check{\nu}\left(\frac{\partial \omega}{\partial x_{j}}\right)^{2}+\omega \frac{\partial \check{\nu}}{\partial x_{j}} \frac{\partial \omega}{\partial x_{j}}\right)\right] \\
& \beta=\beta_{c} f_{\beta} \quad f_{\beta}=\frac{1+64 \psi_{\omega}}{1+80 \psi_{\omega}} \quad \psi_{\omega}=\left|\frac{\Omega_{i j} \Omega_{j k} S_{k i}}{\left(\beta_{0}^{*} \omega\right)^{3}}\right| \quad \omega=\frac{1}{\sqrt{\beta_{c}^{*}}} \sqrt{2 S_{i j} S_{i j}} \quad .
\end{aligned}
$$

The initial and boundary conditions for $\check{\nu}$ are similar to the SPALART-ALLMARAS model

$$
\check{\nu}_{\text {init }} \approx 0.1 \nu \quad \check{\nu}_{\text {wall }}=\left.0 \quad \frac{\partial \check{\nu}}{\partial \vec{n}}\right|_{\text {inflow/outflow }}=0
$$

with the initial turbulent kinetic energy $k_{\text {init }}=\left.\frac{3}{2} u_{\infty}^{2} T u^{2}\right|_{\text {init }}$.

\begin{tabular}{|l|c|c|}
\hline Term & $S A$ Model & new Model \\
\hline \hline Production & $c_{b 1} \check{\nu}\left(\sqrt{2 \Omega_{i j} \Omega_{i j}}+\frac{\check{\nu}}{\kappa^{2} d^{2}}\right)$ & $2(1-\alpha) \check{\nu}\left(\frac{S_{i j}}{\omega} \frac{\partial u_{i}}{\partial x_{j}}-\frac{1}{3} \delta_{i j} \frac{\partial u_{i}}{\partial x_{j}}\right)$ \\
\hline Destruction & $c_{w 1} f_{w}\left(\frac{\check{\nu}}{d}\right)^{2}$ & $\left(\beta^{*}-\beta\right) \check{\nu} \omega$ \\
\hline Diffusion 1 & $\frac{1}{\sigma} \frac{\partial}{\partial x_{j}}\left[(\nu+\check{\nu}) \frac{\partial \check{\nu}}{\partial x_{j}}\right]$ & $\frac{\partial}{\partial x_{j}}\left[(\nu+\sigma \check{\nu}) \frac{\partial \check{\nu}}{\partial x_{j}}\right]$ \\
\hline Diffusion2/Destruction & $\frac{c_{b 2}}{\sigma} \frac{\partial \check{\nu}}{\partial x_{j}} \frac{\partial \check{\nu}}{\partial x_{j}}$ & $2 \frac{(\nu+\sigma \check{\nu})}{\omega} \frac{\partial \check{\nu}}{\partial x_{j}} \frac{\partial \omega}{\partial x_{j}}$ \\
\hline Decay & - & $k_{i n i t}\left(\phi \frac{\check{\nu}}{\nu_{i n i t}}\right)$ \\
\hline
\end{tabular}

Table 3.1: Comparison between the right-hand side terms of the $S A$ and the new model

There are many similarities between the SpAlART-Allmaras model and the the new one-equation model like the same transformation from $\nu_{t}$ to $\check{\nu}$ and the same convective term, which describes the convective transport effect. In table 3.1 the right-hand side terms of both turbulence models are juxtaposed. Turbulence is produced in the $S A$ model mainly through vorticity whereas in the new model it is produced through shear stress. For a 2D free shear layer the production term in both models reduces to $\sim \partial u / \partial y$ but with different proportionality factors. The destruction terms are completely different, since the dependence on the wall distance $d$ is absent in the new 
model. The new model have a much difficult formulation of the destruction term determined by the closure functions $f_{\beta}^{*}, f_{\beta}, \psi_{k}, \psi_{\omega}$. The first diffusion term is almost identical in both models except for coefficients. A second diffusion term is dependent only on the gradient of $\check{\nu}$ in the $S A$ model but is dependent also on the gradient of $\omega$ in the new model. A careful investigation of the term $2 \frac{(\nu+\sigma \check{\nu})}{\omega} \frac{\partial \check{\nu}}{\partial x_{j}} \frac{\partial \omega}{\partial x_{j}}$ shows that this term is always negative, i.e. this term acts as a destruction term. Note that the quantity $\omega$ reconstructed through shear stress plays a major role in production, destruction and diffusion. Finally the new model is extended to account for decay of turbulence, a feature that is missing in the SPALART-Allmaras model.

\subsection{Wall Distance Approximation}

Considering the formulations of turbulence models like the BALDWIN-LOMAX [1], Baldwin-Barth [2], Spalart-Allmaras [110], Secundov's $\nu_{t_{92}}$ and Menter's $S S T$ [63] model it is apparent, that the near-wall turbulence is often considered by explicitly enforcing a dependence of the production or destruction terms, e.g. wall damping terms, on the normal distance $d$ to the nearest wall or on the normalized distance $d^{+}=u_{\tau} d / \nu=\sqrt{\tau_{w} / \rho_{w}} d / \nu$. SPALART argues in $[110,114]$ that $d$ should be interpreted as the normal distance to the wall and not be approximated numerically by the arc length of the grid lines normal to the wall. Nevertheless, there are still situations, where this definition is physically questionable [28] and mathematically ambiguous like at sharp corners.

All the turbulence models mentioned formerly include empirical constants that have to be tuned for the near wall turbulence, which is usually done for the zero pressure gradient boundary layer or slightly curved surfaces. It goes without saying that this procedure is not exact for geometries with strong curvature. The question arises whether a different formulation of the distance can be found, that can take into account curvature effects at strong curved surfaces and sharp edges and corners while preserving the results for the flat plate. This is motivated by the idea that an exact computation of the distance leads to the same result of the distance $d_{1}=d_{2}=d_{3}$ for the convex/concave and plane surfaces shown in figure 3.2. Even though the points $P_{1}, P_{2}, P_{3}$ have the same wall distance they possess a different near-wall impact in many turbulence models. Hence, a physically more rele-

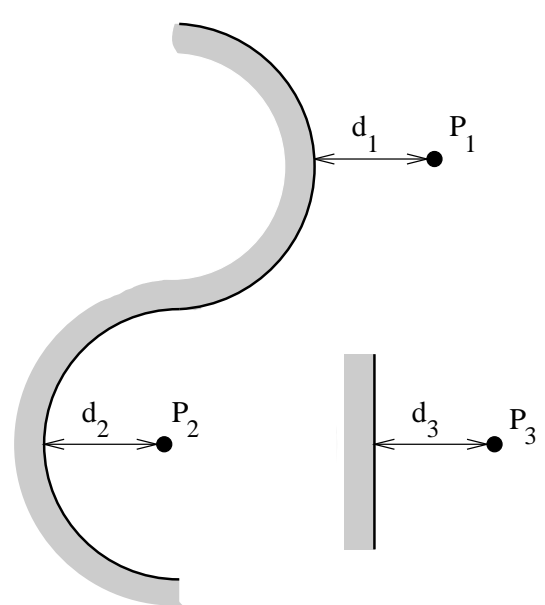

Figure 3.2: Example of equal distances between a surface and a point vant choice would be $d_{1}<d_{3}<d_{2}$ to express the increase or decrease in wall influence at convex or concave curved surfaces. 
Discussions with other researchers $[102,113,128]$ involved in similar topics motivated the development of a new PDE to approximate the wall distance $d$. Such a formulation can be found in $[126,127]$ which was derived from a modified Poisson equation. Several arguments and weighting factors have to be evaluated to identify the nearest surface and locate the nearest corner and gap orientation. Another fast algorithm was presented by TSAI [129] based on geometrical considerations but is limited to analytic or parameterized surfaces.

The formulation derived in this study has computational advantages and can be favorably incorporated into one- and two-equation turbulence models.

The distance function $\mathcal{D}$ for a three-dimensional space is derived from the condition that the gradient of such a function has norm 1

$$
\begin{gathered}
|\nabla \mathcal{D}|=\left|\left(\mathcal{D}_{x}, \mathcal{D}_{y}, \mathcal{D}_{z}\right)^{T}\right|=1 \\
\Rightarrow\left(\mathcal{D}_{x}\right)^{2}+\left(\mathcal{D}_{y}\right)^{2}+\left(\mathcal{D}_{z}\right)^{2}=1,
\end{gathered}
$$

which is the requirement that the function $\mathcal{D}$ has a linear solution. The second condition is that the gradient of $\mathcal{D}$ should be normal to the surface. This is automatically fulfilled by enforcing the Dirichlet boundary condition on the surface

$$
\mathcal{D}_{\text {wall }}=\mathcal{D}_{0}=\text { const }
$$

If the gradient is non-orthogonal, the tangential component on the surface would contradict either the constant boundary condition or the constant norm of the gradient. The validity of the above formulation can be analytically verified for trivial geometries, say, a plane or a sphere.

Note, however, that the numerical solution of the distance equation (3.42) has some drawbacks:

- The initialization of the distribution at the beginning of the calculation by a large constant value will lead to a non-zero residual, even when all boundary conditions are turned off. Only a linear distribution in one direction fulfills the equation. Compared with an initialized parallel flow it seems favorable to have an initialization of the distance equation that indicates convergence either globally or at least locally if there is no wall in the domain or it is far away, respectively.

- The information about the existence of surfaces must propagate through the whole computational domain to attain the linear distribution. This can be avoided since the dependence on the distance $d$ in almost all cases is only important in the vicinity of the surfaces involved.

- If the integration domain is not bounded by walls a suitable distribution of the distance function is not possible except for the infinite value $\mathcal{D} \rightarrow \infty$, which should be avoided numerically. 
Therefore, equation (3.42) is reformulated to overcome these difficulties by substituting the function $\mathcal{D}$ by its inverse $\mathcal{G}=1 / \mathcal{D}$

$$
\begin{aligned}
\left(\mathcal{G}_{x}\right)^{2}+\left(\mathcal{G}_{y}\right)^{2}+\left(\mathcal{G}_{z}\right)^{2} & =\mathcal{G}^{4} \\
\Rightarrow|\nabla \mathcal{G}|^{2} & =\mathcal{G}^{4} \\
\mathcal{G}_{\text {init }} & =0.0 \\
\mathcal{G}_{\text {wall }}=1 / \mathcal{D}_{0} & =\mathcal{G}_{0}
\end{aligned}
$$

To avoid infinite values $\mathcal{D}_{0}$ should not be equal to zero in the boundary condition (3.46) if it is evaluated explicitly at the boundary. The initial distribution in the interior domain is given by equation (3.45), which represents a trivial solution of equation (3.44). The term $|\nabla \mathcal{G}|^{2}$ is rearranged using the identity

$$
\left(\mathcal{G} \cdot \mathcal{G}_{x}\right)_{x}=\left(\mathcal{G}_{x}\right)^{2}+\mathcal{G} \cdot \mathcal{G}_{x x}
$$

for each component of the term to attain an elliptical formulation that is easier to solve numerically

$$
\left(\mathcal{G} \cdot \mathcal{G}_{x}\right)_{x}+\left(\mathcal{G} \cdot \mathcal{G}_{y}\right)_{y}+\left(\mathcal{G} \cdot \mathcal{G}_{z}\right)_{z}-\mathcal{G} \cdot\left(\mathcal{G}_{x x}+\mathcal{G}_{y y}+\mathcal{G}_{z z}\right)-\mathcal{G}^{4}=0
$$

where $\left(\mathcal{G}_{x x}+\mathcal{G}_{y y}+\mathcal{G}_{z z}\right)$ represents the Laplacian of $\mathcal{G}$. To have the desired possibility of tuning the distance function near edges and corners an elliptic term $\varsigma \cdot \mathcal{G}\left(\mathcal{G}_{x x}+\mathcal{G}_{y y}+\mathcal{G}_{z z}\right)$ is added. The quantity $\varsigma$ must be positive since the introduced term resembles a diffusion term. The source term $\mathcal{G}^{4}$ is multiplied by the factor $1+2 \varsigma$ to compensate for the newly added elliptic term and to enforce the exact wall distance calculation for the flat plate. The final equation reads

$$
\nabla \cdot(\mathcal{G} \nabla \mathcal{G})+(\varsigma-1) \mathcal{G} \Delta \mathcal{G}-(1+2 \varsigma) \cdot \mathcal{G}^{4}=0
$$

with

$$
\varsigma \gtrsim 0.2 \quad d=\mathcal{D}-\mathcal{D}_{0}=\frac{1}{\mathcal{G}}-\frac{1}{\mathcal{G}_{0}}
$$

and the initial and boundary conditions

$$
\mathcal{G}_{\text {init }}=0.001 \cdot 1 / L_{\text {ref }} \quad \mathcal{G}_{\text {wall }}=\mathcal{G}_{0} \approx 1 \cdot 1 / L_{\text {ref }}
$$

and $\mathcal{G}_{\text {inflow/outflow }}$ given by an approximate extrapolation of $1 / \mathcal{G}[28]$.

Depending on the value of $\varsigma$ several limiting equations are derived, with $\varsigma=0$ leading to the exact normal distance $d$ and $\varsigma \rightarrow \infty$ leading to a regular POISSON equation. A value of $\varsigma=1$ is suggested for the calculation [28], which enhances numerical stability of the scheme. The numerical solution of the discrete system of equations can be achieved efficiently by a NEWTON type iteration as described in [28].

An example illustrating the distance calculation of a three-element airfoil BAC 3-11 [65] investigated in later simulations is presented. The exact distance based on the calculation of the smallest distance between the grid points within the integration domain and the boundary points on the wall is presented in figure 3.3. Note the relatively sharp distribution in the vicinity of the slat and flap, that results in perturbations in the 
numerical computation of the turbulent flow. The distance calculation based on the proposed PDE in figure 3.4 shows a comparatively smooth distribution. Note that in the direct vicinity of the wall, i.e. within the viscous sublayer, the distance distribution calculated through equation (3.48) gives a good approximation to the exact distance. Depending on the value of $\varsigma$ and $\mathcal{G}_{0}$ the region where the approximation between the exact distance and the proposed PDE can be controlled. For details on the approximation please refer to [28].

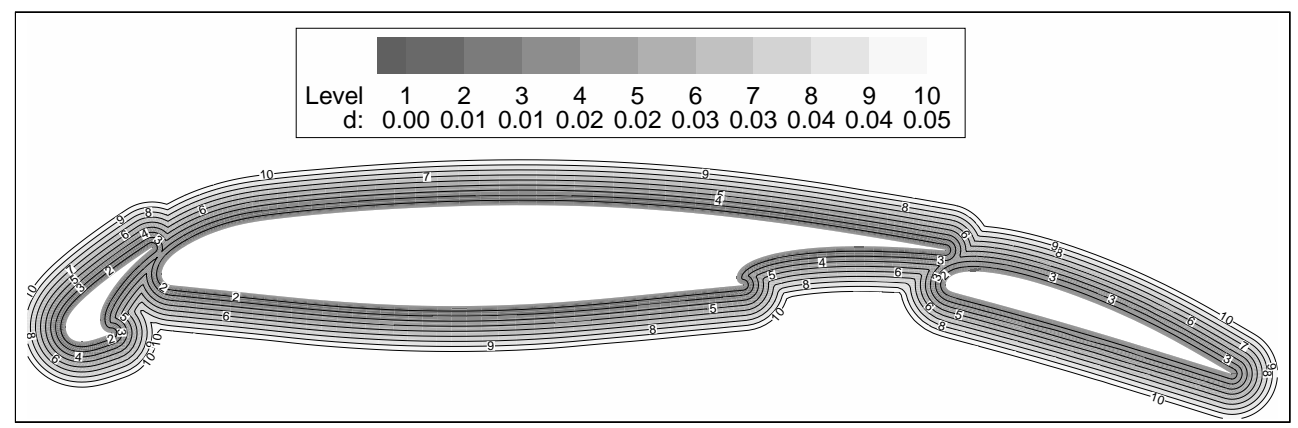

Figure 3.3: $\quad$ Exact distance

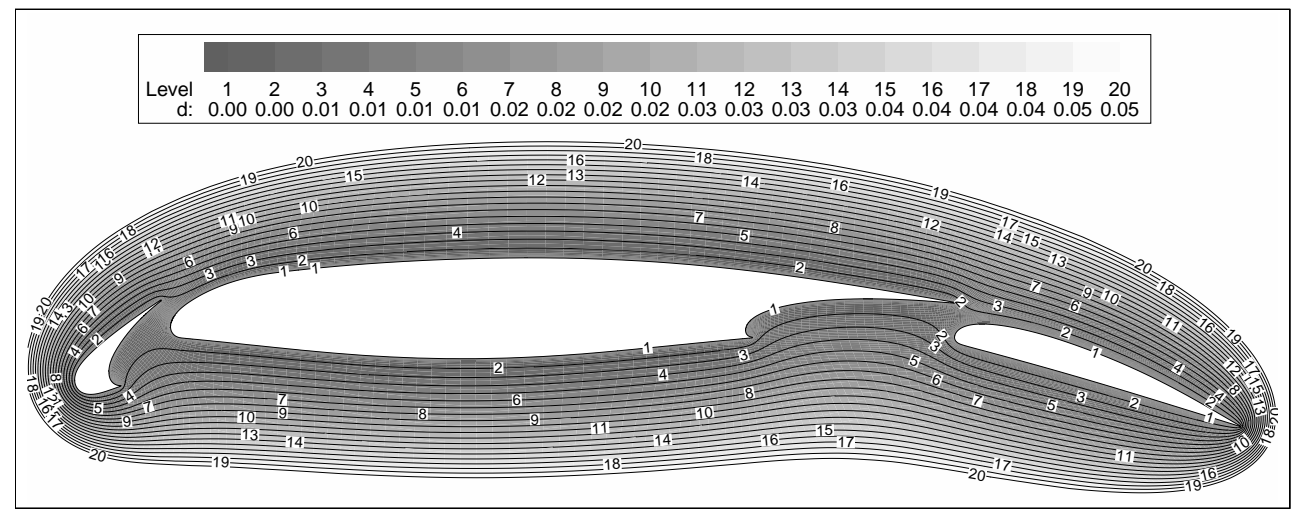

Figure 3.4: Calculated distance with $\varsigma=1.0$ and $\mathcal{G}_{0}=1.0$

In addition to the physical relevance of the new formulation there are also numerical advantages over the exact calculation of the distance $d$, especially in the framework of three-dimensional multiblock topologies or unstructured meshes. This usually involves a complex and time-consuming search algorithm that is very difficult to implement efficiently on parallel or vector computers [28]. Figure 3.5 presents the computational time needed for the exact calculation of $d$ in one block consisting of $N$ grid points and the computational time required for the convergence of the numerical method of the proposed distance equation which is defined by a drop of $\mathcal{O}(7)$ magnitudes of the residual. Additionally, figure 3.6 shows an example of the scaling of the computational time with growing block and processor number $K$, respectively. The size of the problem $N / K$ was kept equal for each processor. The findings visualized in both figures confirm the efficiency of the numerical solution of the proposed equation compared to the exact distance calculation. 


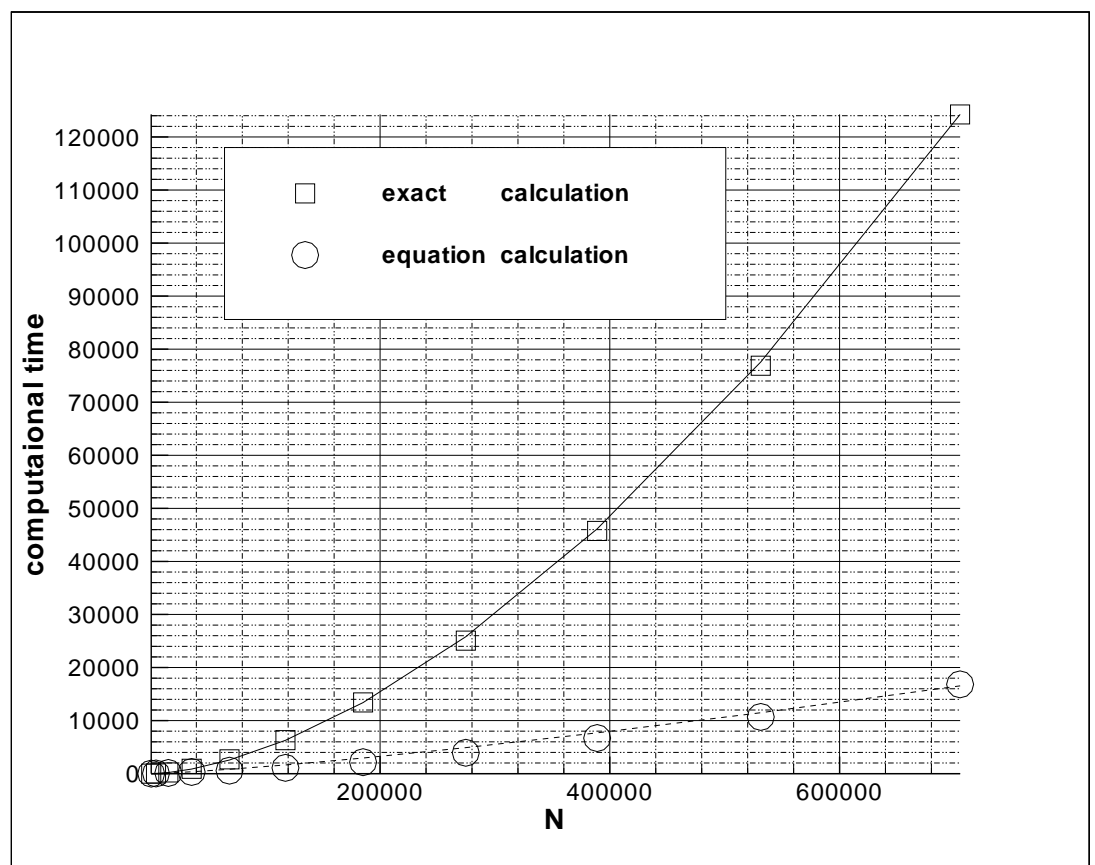

Figure 3.5: Computational time vs. number of grid points $N$ normalized by the time required for the smallest grid with $9 \times 9 \times 9$ grid points

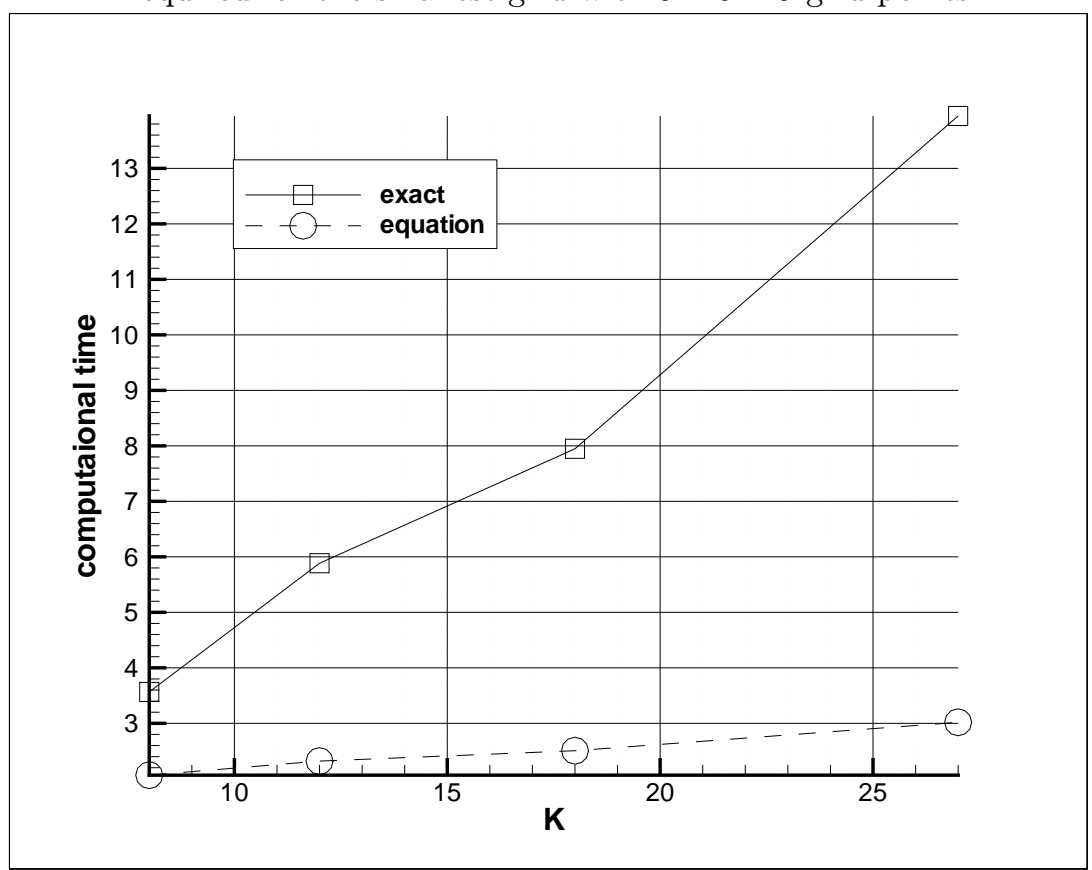

Figure 3.6: Computational time vs. number of blocks $K$ normalized by the time required for one block 


\section{Summary}

In this chapter a short review on one-equation turbulence models was presented. A modified SPALART-ALLMARAS model was discussed and a new one-equation model was derived from the $k-\omega$ two-equation model. The new model has several advantages and promises better predictions than the SPALART-AllmarAs model for free shear turbulent flows. The validation of the new model and comparisons with the SPALARTAllmaras and the $k-\omega$ models is given in chapter 5. Furthermore, a PDE was derived for the calculation of the wall distance, which is needed by some turbulence models. The numerical solution of the new approximate wall distance equation is computationally more efficient than the direct calculation of complex geometries. 


\section{Chapter 4}

\section{Method of Solution}

"It would appear that we have reached the limits of what is possible to achieve with computer technology, although one should be careful with such statements, as they tend to sound pretty silly in five years."

John von Neumann.

In general the governing equations for fluid flow described in chapter 2 cannot be solved analytically. Approximate solutions can be achieved with numerical methods by first discretizing the underlying differential equations and then solving the resulting discrete system using computers. The approximation does imply the discretization of space and time such that the numerical solution provides results at discrete locations in space and time. The fundamental equivalence theorem of LAX [84] proves for linear equations that consistency and stability of the discretization scheme are necessary and sufficient conditions for the convergence of the numerical scheme to the exact solution of the differential equation.

The numerical method presented in the following is similar to the JAMESON et al. multi-stage method [47], which is widely used for simulating compressible aerodynamic flows [41].

First the finite volume method is presented followed by a description of the spatial and temporal discretization scheme used in this work. Different techniques for acceleration to steady state solutions are shown in chapter 4.4. Finally the numerical initial and boundary conditions based on different approaches are given in chapter 4.5.

\subsection{Finite Volume Method}

The starting point of the discretization of the NAVIER-STOKES equations is the integral form of the equations (2.3). The computational domain is divided into contiguous finite number of control volumes. The conservation equations are then applied to 
each discrete control volume. This approach guarantees conservation of conservative variables, e.g. specific energy $\rho e$, and is therefore applicable also for flows with discontinuities like shocks and contact discontinuities. All flow variables are located at the centers of control volumes to be defined later in chapter 4.1.2. For the calculation of the surface integrals the fluxes are needed at the cell faces or corners which is achieved through interpolation of the centroid values at the required locations. To achieve a second-order global accuracy of the numerical solution each of the discretization, interpolation, integration and further approximation schemes must have truncation errors consistent with the second-order accuracy.

\subsubsection{Boundary-Fitted Structured Grid}

The control volumes used to discretize the domain of integration in three-dimensional space are chosen to be hexahedras arranged in a structured manner such that a transformation from the equidistant cartesian computational domain to curvilinear physical space and vice versa as presented in figure 4.1 is possible. This allows a generation of a boundary-fitted grid that enables an easy and accurate implementation of the boundary conditions.
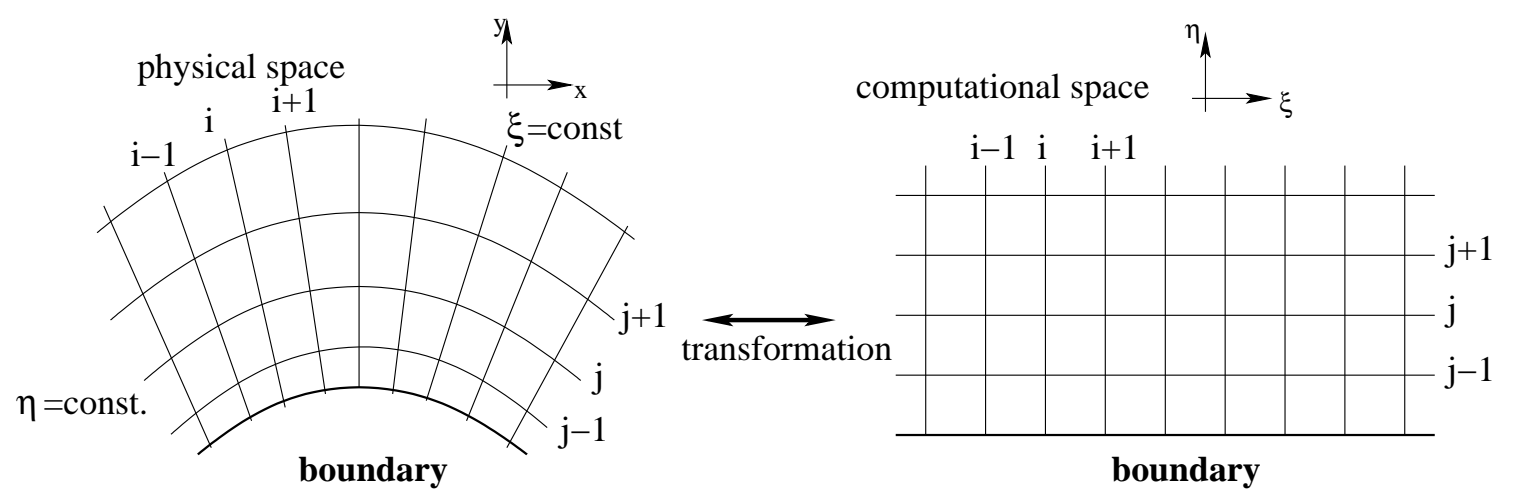

Figure 4.1: Coordinate transformation in two-dimensional space

The transformation presented in appendix $\mathrm{D}$ is chosen such that $\triangle \xi=\triangle \eta=\triangle \zeta=1$. The determinant of the transformation matrix $J$ (D.4) can be interpreted as the cell volume in physical space. The metric relations (D.3) are calculated numerically with a second-order central scheme using the physical coordinates of the grid points, e.g.

$$
y_{\xi}=\frac{y^{i+1}-y^{i-1}}{\xi^{i+1}-\xi^{i-1}}=\frac{1}{2}\left(y^{i+1}-y^{i-1}\right) .
$$

Note that the second-order accuracy of the whole numerical scheme is preserved only if the grid is smooth and almost orthogonal [40] otherwise it can result in local loss of accuracy.

The conservation properties of the integral form (2.3) are maintained in the transformed equations (E.1) if the flux $\vec{F}$ remains the same for the cell face in both control volumes 
sharing this cell face. This approach corresponds to an approximation of volume and surface integrals in equation (2.3) using the midpoint rule [30], which is of second-order accuracy.

\subsubsection{Node Centered Scheme}

The flow variables are located at the nodes of the computational grid. The corresponding control volume is shown in figure 4.2. Other variants of variable arrangements and control volumes do exist $[30,40]$ but are not discussed below.

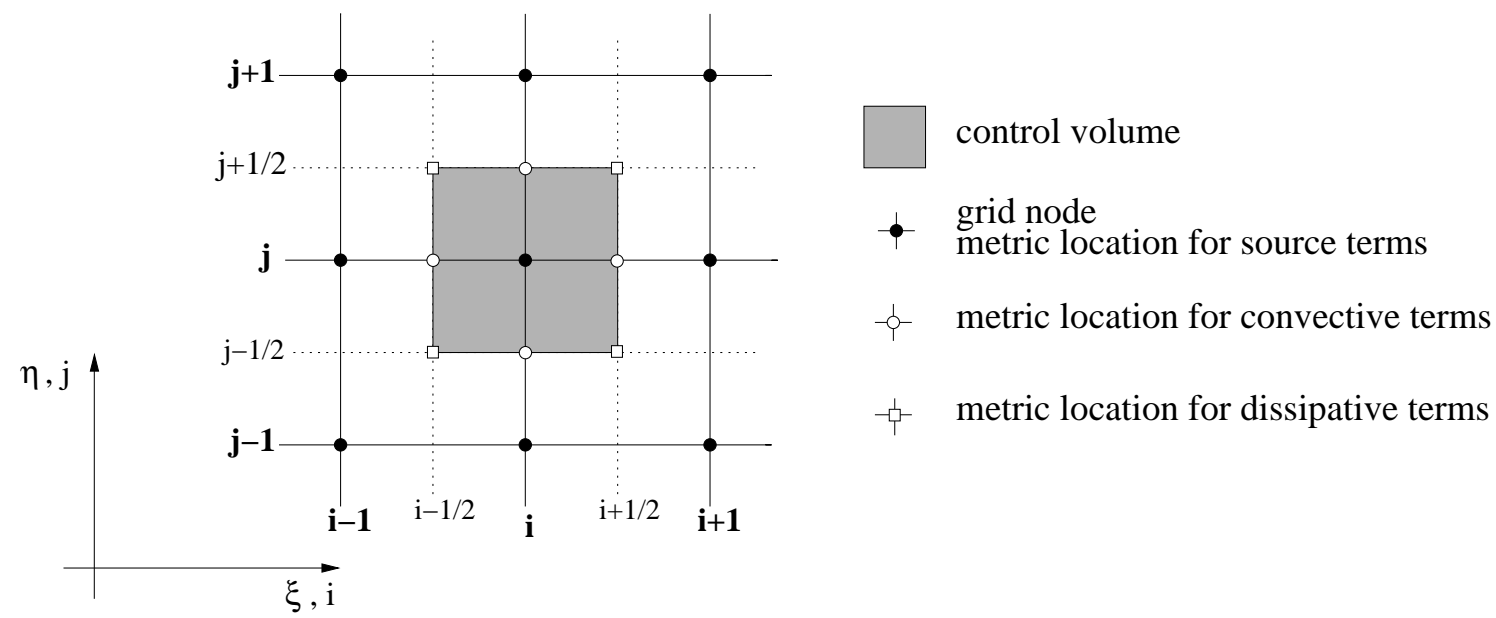

Figure 4.2: Control volume of node centered scheme with different metric locations in two-dimensional space

The calculation of the advective terms requires the evaluation of the fluxes at the cell faces. The choice of the metric terms $\left(\xi_{x}, \eta_{x} \ldots\right)$ located at the midpoints $i \pm 1 / 2, j$ and $i, j \pm 1 / 2$ as shown in figure 4.2 is suitable for the reconstruction of left and right values used in the approximation presented in chapter 4.2.1 and appendix F. The dissipative terms contain second-order derivatives needed at the cell corners of the control volume that lead to the definition of the metric terms at the corners, e.g. $i \pm 1 / 2, j \pm 1 / 2$ as shown in figure 4.2, which is discussed in chapter 4.2.2, whereas the source and also the corresponding metric terms are defined at the cell center, e.g. $i, j$ in figure 4.2 , as discussed in chapter 4.2.3.

\subsubsection{Multiblock Arrangement and Parallelization}

The generation of structured grids around complex geometries is impossible without the multiblock concept. An example of such a multiblock grid is presented in figure 4.3. The idea is to use a regular data structure within each block while the blocks can be 
arranged in an unstructured order that allows the generation of smooth and near orthogonal grids even for complex and irregular geometries. The grid lines of each block overlap at the block boundaries. That is the grid points at a multiblock boundary are identical in both blocks, which considerably simplifies the multiblock treatment at these boundaries.

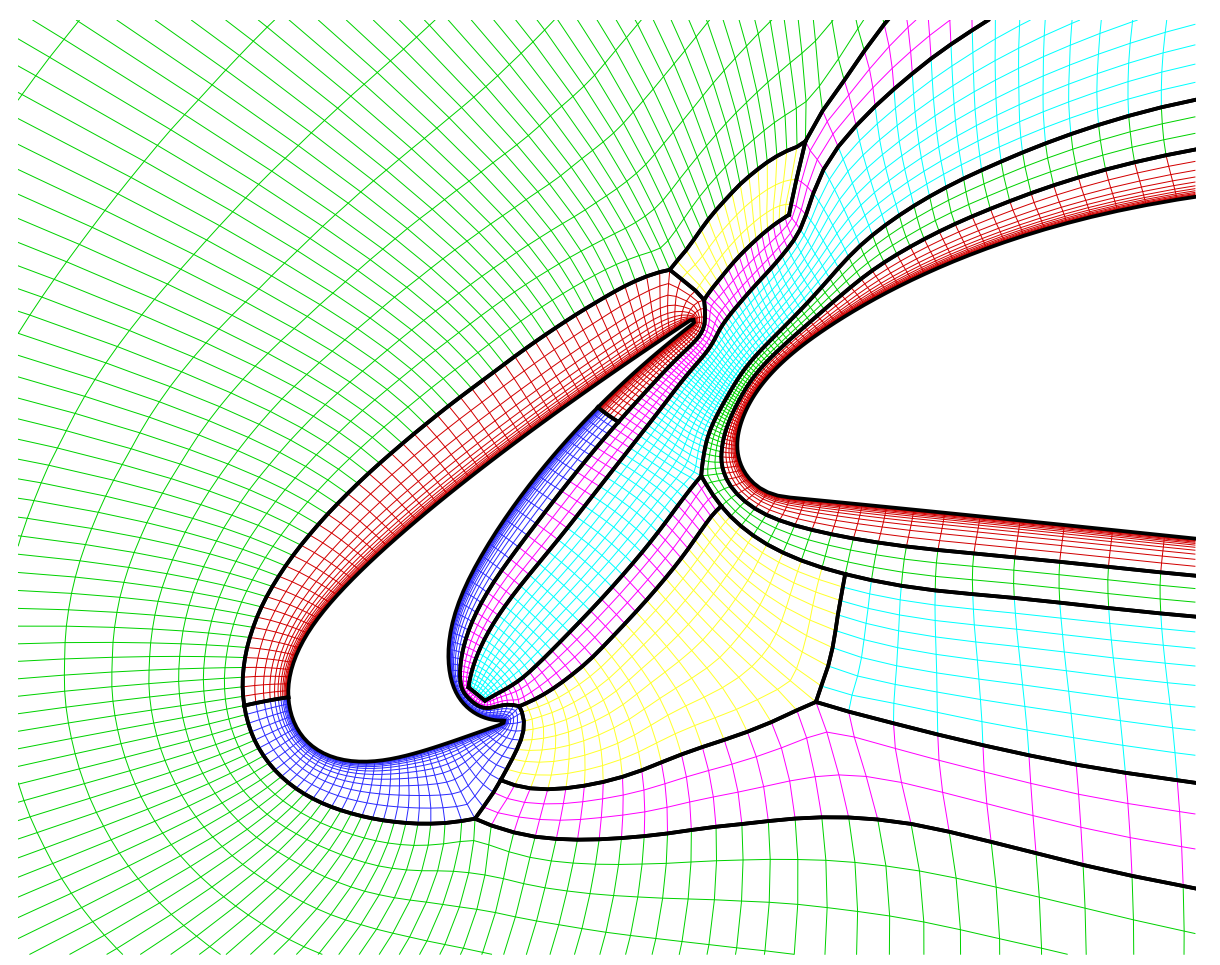

Figure 4.3: Two-dimensional multiblock grid of a three element airfoil near the slat

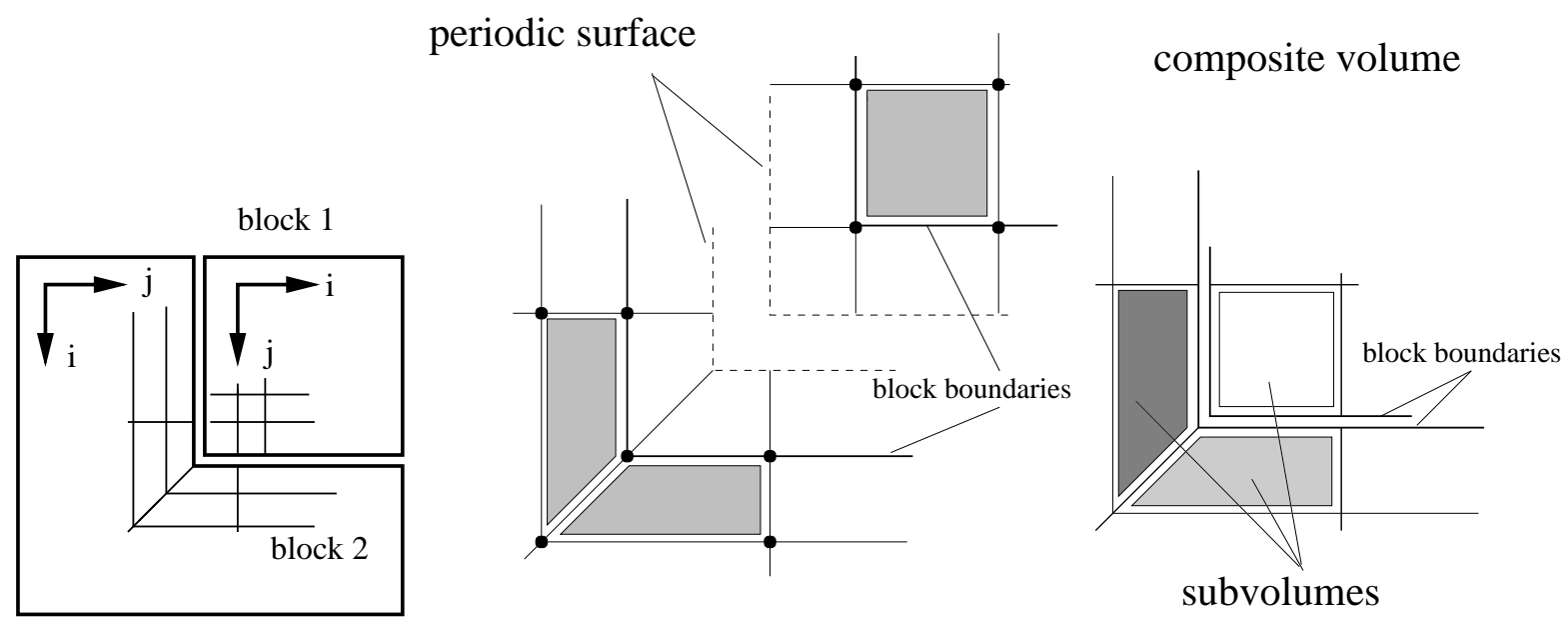

Figure 4.4: Multiblock treatment at irregular boundaries

To guarantee the conservative character of the solution at the multiblock boundaries the values at corresponding nodes in both blocks must be the same. The blocks are extended through periodic surfaces as indicated in figure 4.4 that accommodate the 
values at interior locations of the neighboring block. The values on the periodic surfaces are necessary for each block to interpolate variables and to calculate the fluxes near the boundary. In case of an irregular boundary like a corner as that presented in figure 4.4 or even more complex multiblock topologies in three-dimensional space, conservation is achieved by subdividing the control volumes at block boundaries into subvolumes. The fluxes are calculated at the surfaces of these composite cells within each block and then summed up to give the total flux. Similarly, the source terms are calculated separately in each block and then multiplied by each subvolume and summed up afterwards.

The exchange of data on the periodic surfaces between the blocks is performed according to a send and receive communication, such that the additional information of neighboring blocks is copied into the data structure of each block. The calculation within each block can therefore be done separately for one iteration step. The subsequent summation of the fluxes and source parts is also distributed over all involved blocks to complete the iteration step. This approach coincides with the domain decomposition technique implemented for parallel execution, since all blocks are independent objects that can be assigned to separate processors and calculated simultaneously. The communication is achieved through the message passing library MPI [37].

\subsection{Spatial Discretization}

The discrete approximation of the spatial derivatives of the NAVIER-STOKES equations depends on the character of the terms to be discretized. The advective fluxes are hyperbolic and thus have real characteristics that define the direction, speed of propagation and the regions of dependence. The dissipative fluxes have an elliptic character with information traveling with virtually infinite speed in all directions. The source terms introduced by the turbulence model involve spatial derivatives, which are usually treated similar to dissipative fluxes.

\subsubsection{Discretization of the Advective Terms}

Central schemes of second-order accuracy like the Lax-Wendroff schemes $[52,53]$ used for the discretization of the advective terms do not take into account the propagation of information and perturbations along the characteristics. This leads to oscillations in the solution and instabilities of the scheme that can be suppressed numerically by the addition of artificial dissipation terms [41, 47].

Another class of discretization schemes that inherently exploit the physical properties of the advective terms are often called upwind methods, for an overview see [41, 89]. The flux difference splitting FDS schemes derived from GodunOv's method [34] contain an approximate solution of the local RIEMANN problem at the cell faces, whereas the flux vector splitting $F V S$ schemes split the flux terms into a left and a right part and discretize them according to the sign of the associated propagation speeds. Generally speaking the $F V S$ schemes are simpler in the formulation and more efficient in the 
implementation than the $F D S$ schemes but they possess a higher numerical dissipation that prohibit sharp or accurate resolution of discontinuities and shear layers. The advection upwind splitting method AUSM [58] and its derivative AUSMDV [132] used in this work combines advantages of both schemes. The implemented version of the scheme is given in appendix F.

\section{Variable Reconstruction}

The required left ()$_{L}$ and right ()$_{R}$ states of a flow variable $\Phi$ at a location $i+1 / 2$ are interpolated from neighboring values as sketched in figure 4.5 according to the monotonic upstream centered scheme for conservation laws $M U S C L$ of VAN LEER [54] to achieve a second-order accuracy of the scheme. A flux limiter like that of VAN ALBADA [130] can be used to suppress oscillations near discontinuities and extrema by locally reducing the order of the reconstruction to first-order. Since no discontinuities are encountered in this work no limiter was used.

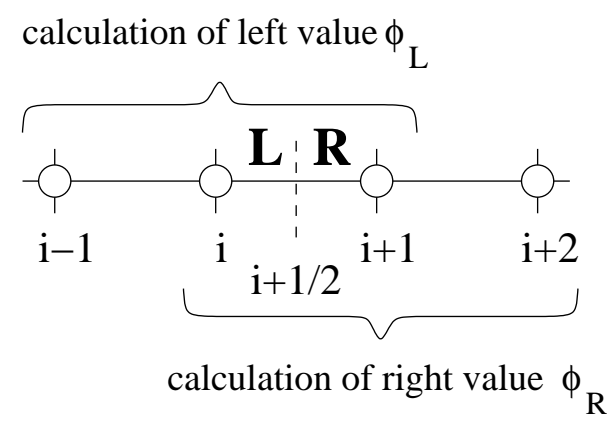

Figure 4.5: Interpolation of left $\mathbf{L}$ and right $\mathbf{R}$ states at position $i+1 / 2$

Higher order interpolations for the reconstruction of the variables were investigated in [27] for the wake flow to achieve a more accurate resolution of vortices. The reconstruction scheme with one downwind and two upwind points as presented in figure 4.5 leading to a third-order accurate scheme was found to be the best compromise between accuracy and stability. The use of this scheme reduced the numerical dissipation considerably and as such the number of grid points for the wake flow could be decreased by $\mathcal{O}(10)$ compared to the standard second-order accurate schemes as shown in chapter 5.1.2. The used reconstruction schemes are given in appendix F.

\subsubsection{Discretization of the Dissipative Terms}

The dissipative terms are discretized to second-order accuracy by central differences.

The flux $\overrightarrow{\vec{E}}_{D}$ in curvilinear coordinates has terms similar to $\mu \xi_{x} \xi_{y} u_{\xi}$ that are discretized 
e.g. at the cell face position $i+1 / 2, j, k$ according to ${ }^{1}$

$$
\begin{aligned}
\left.\mu \xi_{x} \xi_{y} u_{\xi}\right|^{i+1 / 2, j, k}= & \frac{1}{4}\left(\left.\mu \xi_{x} \xi_{y} u_{\xi}\right|^{i+1 / 2, j+1 / 2, k+1 / 2}+\left.\mu \xi_{x} \xi_{y} u_{\xi}\right|^{i+1 / 2, j+1 / 2, k-1 / 2}\right. \\
& \left.+\left.\mu \xi_{x} \xi_{y} u_{\xi}\right|^{i+1 / 2, j-1 / 2, k+1 / 2}+\left.\mu \xi_{x} \xi_{y} u_{\xi}\right|^{i+1 / 2, j-1 / 2, k-1 / 2}\right)
\end{aligned}
$$

with

$$
\begin{aligned}
\left.u_{\xi}\right|^{i+1 / 2, j+1 / 2, k+1 / 2}= & \frac{1}{4}\left[\left(u^{i+1, j, k}+u^{i+1, j+1, k}+u^{i+1, j, k+1}+u^{i+1, j+1, k+1}\right)\right. \\
& \left.-\left(u^{i, j, k}+u^{i, j+1, k}+u^{i, j, k+1}+u^{i, j+1, k+1}\right)\right]
\end{aligned}
$$

and $\mu^{i+1 / 2, j+1 / 2, k+1 / 2}$ as the average of $\mu$ on the cell corner

$$
\begin{array}{ll}
\mu^{i+1 / 2, j+1 / 2, k+1 / 2}=\frac{1}{8} \quad & \left(\mu^{i, j, k}+\mu^{i+1, j, k}+\mu^{i, j+1, k}+\mu^{i, j, k+1}\right. \\
& \left.+\mu^{i+1, j+1, k}+\mu^{i+1, j, k+1}+\mu^{i, j+1, k+1}+\mu^{i+1, j+1, k+1}\right)
\end{array}
$$

The advantage of this central discretization scheme is that the metric terms are needed only at the positions $i \pm 1 / 2, j \pm 1 / 2, k \pm 1 / 2$ and that the ScHWARz rule for cross derivatives is automatically satisfied due to the symmetry of the numerical operations, i.e. the cross derivative is independent of the order of derivation, which is assumed through $\tau_{x y}=\tau_{y x}, \tau_{x z}=\tau_{z x}$ and $\tau_{y z}=\tau_{z y}$.

\subsubsection{Discretization of the Source Terms}

The source terms contain products of first derivatives like $\xi_{x} u_{\xi}$ that are discretized e.g. at position $i$ using central differences of second-order accuracy

$$
\left.\xi_{x} u_{\xi}\right|^{i}=\frac{1}{2} \xi_{x}^{i}\left(u^{i+1}-u^{i-1}\right)
$$

\subsection{Temporal Discretization}

The discretization of the spatial gradients of the fluxes and the source terms at a discrete time level $n$ are denoted by the residual $\operatorname{Res}\left(\vec{Q}^{n}\right)$. Inserted in the underlying $P D E$ they lead to the equation

$$
\left.\frac{\partial \vec{Q}}{\partial t}\right|^{n}=\operatorname{Res}\left(\vec{Q}^{n}\right)
$$

which can be interpreted as an initial value problem of an ordinary differential equation. Depending on the discretization of the time derivative several explicit and implicit time

\footnotetext{
${ }^{1}$ all other terms and directions are similar
} 
integration schemes can be derived, for an overview see [41]. Implicit methods generally offer more stable schemes but require considerably more memory and computation resources per time step than explicit schemes, which complicates their efficient application for three-dimensional calculations over complex geometries. The time stepping scheme used here to advance the discrete numerical representation over a time interval $\triangle t$

$$
\frac{\vec{Q}^{n+1}-\vec{Q}^{n}}{\triangle t}=\operatorname{Res}\left(\vec{Q}^{n}\right)
$$

is an explicit 5-stage RUNGE-KUTTA integration scheme

$$
\begin{aligned}
\vec{Q}^{(0)} & =\vec{Q}^{n} \\
\vec{Q}^{(1)} & =\vec{Q}^{(0)}+\grave{\alpha}_{1} \cdot \Delta t \cdot \operatorname{Res}\left(\vec{Q}^{(0)}\right) \\
\vdots & \\
\vec{Q}^{(m)} & =\vec{Q}^{(0)}+\grave{\alpha}_{m} \cdot \Delta t \cdot \operatorname{Res}\left(\vec{Q}^{(m-1)}\right) \\
\vdots & \\
\vec{Q}^{n+1} & =\vec{Q}^{(5)} .
\end{aligned}
$$

The coefficients $\grave{\alpha}_{m}$ are optimized for maximum stability of the numerical scheme using the $A U S M$ upwind scheme for a linearized model equation according to [103]

$$
\grave{\alpha}_{m=1 . .5}=(0.059,0.140,0.273,0.500,1.00)
$$

Unlike in classical multistep RUNGE-KUTTA schemes each stage $m$ of the integration steps is independent from all previous stages which is why the accuracy of the proposed scheme is only of second-order. The advantage of this scheme is that only two states $\vec{Q}^{(0)}$ and $\vec{Q}^{(m)}$ have to be simultaneously stored.

A stability analysis of the proposed numerical scheme shows that the maximum time increment $\Delta t$ is determined by a maximum COURANT-FRIEDRICHS-LEVY number $C F L_{\max } \leq 3.5[60,103]$, where $C F L$ is defined for a one-dimensional compressible viscous flow in the $x$-direction as

$$
C F L_{1 D}=\triangle t\left(\frac{4 \nu}{\triangle x^{2}}+\frac{|u|+a}{\triangle x}\right)
$$

and generalized for a three-dimensional flow using the contravariant velocities $U, V, W$ and the metric terms described in appendix D

with

$$
C F L_{3 D}=\Delta t\left(\frac{2 H_{\xi \eta \zeta}}{R e_{0}}+|U|+|V|+|W|+a G_{\xi \eta \zeta}\right)
$$

$$
\begin{aligned}
H_{\xi \eta \zeta} & =\left(\xi_{x}^{2}+\xi_{y}^{2}+\xi_{z}^{2}+\eta_{x}^{2}+\eta_{y}^{2}+\eta_{z}^{2}+\zeta_{x}^{2}+\zeta_{y}^{2}+\zeta_{z}^{2}\right) \\
G_{\xi \eta \zeta} & =\left(\sqrt{\xi_{x}^{2}+\xi_{y}^{2}+\xi_{z}^{2}}+\sqrt{\eta_{x}^{2}+\eta_{y}^{2}+\eta_{z}^{2}}+\sqrt{\zeta_{x}^{2}+\zeta_{y}^{2}+\zeta_{z}^{2}}\right)
\end{aligned}
$$


Consequently, the maximum time step $\Delta t_{\max }$ is mainly determined by the cell size and the local velocities and the local speed of sound. The limitation through the dissipative term is usually not important, since the REYNOLDS number $R e_{0}$ is in the order of $\mathcal{O}\left(10^{6}\right)$. To retain time accuracy and stability of the scheme the smallest time step in the computational domain determined by the smallest cell, used usually in resolving the boundary layer, must be used throughout the computational domain.

\subsection{Acceleration to Steady State}

Since the flows investigated in this work are assumed steady we do not seek time accurate solutions. The time stepping scheme can therefore be interpreted as an iteration index. Different techniques can be used to accelerate substantially convergence to a steady state $\left.\frac{\partial \vec{Q}}{\partial t}\right|^{n}=\operatorname{Res}\left(\vec{Q}^{n}\right)=0$.

\subsubsection{Local Time Stepping}

Local time stepping utilizes the maximum time step at each grid point during the course of the time integration. Hence, $\Delta t_{\max }$ in equation 4.11 is evaluated locally at each grid cell. The steady-state solution is unaffected by this local time stepping procedure and it can be obtained in much fewer iterations.

\subsubsection{Rational Runge-Kutta Integration Scheme}

A two-stage rational Runge-Kutta scheme described in $[98,133]$ is proposed for steady flows with the following intermediate corrections $\triangle \vec{Q}^{1,2,3}$ at the time level $n$

$$
\begin{aligned}
& \triangle \vec{Q}^{1}=\Delta t \operatorname{Res}\left(\vec{Q}^{n}\right) \\
& \triangle \vec{Q}^{2}=\triangle t \operatorname{Res}\left(\vec{Q}^{n}+\grave{c} \triangle \vec{Q}^{1}\right) \\
& \triangle \vec{Q}^{3}=(1-\grave{b}) \triangle \vec{Q}^{1}+\grave{b} \triangle \vec{Q}^{2}
\end{aligned}
$$

to obtain the solution at time level $n+1$

$$
\vec{Q}^{n+1}=\vec{Q}^{n} \frac{2 \triangle \vec{Q}^{1} \sum_{i}\left(\triangle \vec{Q}_{i}^{1} \triangle \vec{Q}_{i}^{3}\right)-\triangle \vec{Q}^{3} \sum_{i}\left(\triangle \vec{Q}_{i}^{1} \triangle \vec{Q}_{i}^{1}\right)}{\sum_{i}\left(\triangle \vec{Q}_{i}^{3} \triangle \vec{Q}_{i}^{3}\right)}
$$

with $i$ as the grid index and the summation $\sum_{i}$ is performed over all grid points. The coefficients $\{\grave{b}, \grave{c}\}=\{-1 ., 0.5\}$ are chosen to ensure second-order accuracy [98]. The intermediate corrections require an additional storage of three states of $\vec{Q}$. The rational RungE-KUTTA scheme needs only two intermediate steps instead of five for the standard 5 step Runge-KutTa scheme. Since it does increase the stability limit $C F L_{\max }$ by a factor of 2 the convergence is accelerated considerably at the beginning of the calculation. There are, however, situations, where convergence to a steady state 
cannot be achieved using the rational RUNGE-KUTTA scheme, since the weighting factors, e.g. $\sum_{i}\left(\triangle \vec{Q}_{i}^{1} \triangle \vec{Q}_{i}^{3}\right)$ are not averages over all grid points but represent the sum of all changes $\triangle \vec{Q}_{i}$. Therefore, large residuals $\operatorname{Res}(\vec{Q})_{i}$ at a limited number of points especially near boundaries influence substantially the integration scheme at all points and consequently can prohibit convergence to machine accuracy.

\subsubsection{Implicit Residual Smoothing}

The stability range of the time stepping scheme can be enlarged by using the concept of implicit smoothing of the residuals [41, 47], which introduces an implicit character into the time stepping scheme and smoothes high-frequency disturbances leading to instabilities in the solution process. The smoothed residual Res* at a time level $n$ is determined using an elliptical equation

$$
\left(1-\epsilon^{*} \Delta \operatorname{Res}^{*}\left(\vec{Q}^{n}\right)\right)=\operatorname{Res}\left(\vec{Q}^{n}\right)
$$

with a constant coefficient $\epsilon^{*}=0.3$ that has been empirically determined through a series of studies of turbulent flows. The implicit operator $1-\epsilon^{*} \Delta$ is approximated using a factorization in each coordinate direction

$$
\left(1-\epsilon^{*} \Delta\right) \approx\left(1-\epsilon^{*} \Delta_{\xi}\right)\left(1-\epsilon^{*} \Delta_{\eta}\right)\left(1-\epsilon^{*} \Delta_{\zeta}\right)
$$

This allows sequential inversion of the implicit operator in each direction

$$
\begin{aligned}
\operatorname{Res}^{* * *}\left(\vec{Q}^{n}\right) & =\left(1-\epsilon^{*} \Delta_{\xi}\right)^{-1} \operatorname{Res}\left(\vec{Q}^{n}\right) \\
\operatorname{Res}^{* *}\left(\vec{Q}^{n}\right) & =\left(1-\epsilon^{*} \Delta_{\eta}\right)^{-1} \operatorname{Res}^{* * *}\left(\vec{Q}^{n}\right) \\
\operatorname{Res}^{*}\left(\vec{Q}^{n}\right) & =\left(1-\epsilon^{*} \Delta_{\zeta}\right)^{-1} \operatorname{Res}^{* *}\left(\vec{Q}^{n}\right)
\end{aligned}
$$

and hence reduces the original linear system of equations into three separate tridiagonal systems that can be solved efficiently using direct methods like the THOMAS Algorithm [30]. The residual smoothing does not influence the accuracy of the final solution since the residual $\operatorname{Res}\left(\vec{Q}^{n}\right)$ vanishes when the steady state is reached.

\subsubsection{Multigrid Method}

The multigrid method is a numerical solution technique that can be applied to accelerate the convergence of an iterative process. This is achieved by computing corrections to the fine grid solution on coarser meshes and interpolating these changes onto the fine mesh. This operation can be applied recursively at several coarser grid levels. In general the coarse mesh is derived from the preceding finer mesh by eliminating every second mesh line in each coordinate direction. This restricts the number of grid points in one direction to a value $2^{k+1}+1$, which allows a maximum of $k$ coarsening levels. The full-approximation storage $F A S$ multigrid procedure [60] can be applied here with a sawtooth $\mathrm{V}$-cycle strategy consisting of 3 coarsening levels as sketched in figure 4.6. 
mesh level

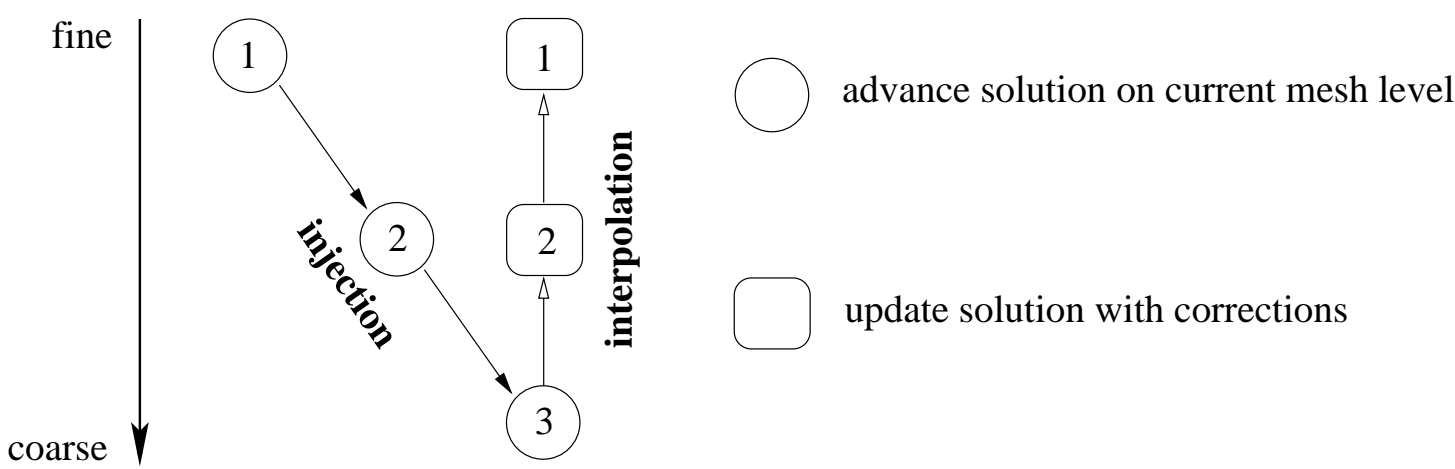

Figure 4.6: Multigrid sawtooth V-cycle

The solution on the fine mesh is injected onto the coarser mesh level where the solution is advanced for one iteration step. The procedure is repeated successively until the coarsest mesh level is reached. At this point, the correction to the solution $\vec{Q}^{n+1}-\vec{Q}^{n}$ is interpolated to the next finer mesh sequentially until the finest mesh is reached. A detailed discussion on the concept of multigrid methods can be found in $[9,10,107]$.

\subsection{Initial and Boundary Conditions}

The physical initial and boundary conditions described in chapter 2.3 are implemented by specifying the values of the conservative variables at the beginning of the calculation $\vec{Q}_{i n i t}$ and at the boundaries $\vec{Q}_{b}$ (or their Residuals $\operatorname{Res}(\vec{Q})_{b}$ ) during the calculation at each time step.

\subsubsection{Initial Conditions}

The initialization of $\vec{Q}$ is usually based on the freestream values at infinity $\rho_{\infty}, p_{\infty}, \vec{v}_{\infty}$ and the initial turbulence value $\check{\nu}_{\text {init }}$ stipulated in equations $(2.20,3.40)$

$$
\vec{Q}_{i n i t}=\vec{Q}_{\infty}=\left(\rho_{\infty}, \rho_{\infty} \vec{v}_{\infty}, \frac{p_{\infty}}{\gamma-1}+\frac{1}{2} \rho_{\infty} \vec{v}_{\infty}^{2}, \rho_{\infty} \check{\nu}_{i n i t}\right)^{T}
$$

\subsubsection{Boundary Conditions}

Investigations of the numerical boundary conditions [38, 41, 79, 124] of the EULER and the NAVIER-Stokes equations at high REynOLDS number evidence the negligible 
physical relevance of the viscous terms in the derivation of consistent and numerically stable boundary conditions at inflow and outflow boundaries. Several conditions, usually based on a one-dimensional analysis, can be derived with growing complexity and accuracy. The derivation of the one-dimensional characteristic form of the EULER equations in curvilinear coordinates used in the formulation of the inflow/outflow boundary conditions is given in appendix $\mathrm{G}$.

\section{No-Slip}

The physical boundary condition in equations $(2.21,3.40)$ is imposed at the grid points on the non-moving wall

$$
\vec{Q}_{\text {wall }}=\left(\rho_{\text {wall }}, 0,0,0, \frac{p_{\text {wall }}}{\gamma-1}, 0\right)^{T}
$$

with $\rho_{\text {wall }}$ and $p_{\text {wall }}$ extrapolated from the first inner point $b-1$ of the adiabatic wall boundary according to the boundary layer simplification

$$
\rho_{\text {wall }}=\rho_{b-1} \quad p_{\text {wall }}=p_{b-1}
$$

The extrapolation $p_{\text {wall }}=p_{b-1}$ is a good approximation to the NEUMANN condition $\left.\frac{\partial p}{\partial \vec{n}}\right|_{\text {wall }}=0$ if grid lines are orthogonal to the wall boundary and have a small spacing, which is usually satisfied for the grids used to resolve the boundary layer at high REYNOLDS numbers.

\section{Inflow/Outflow}

Since usually no exact physical values or analytical solutions can be prescribed at the inflow/outflow boundaries of finite domains three categories of approximations are derived for subsonic flows.

Simple extrapolations: These boundary conditions take into account only the required number of values to be imposed explicitly at the boundaries according to the investigation of the EULER equations. The rest of the values are extrapolated from the interior of the computational domain

$$
\begin{aligned}
\vec{Q}_{\text {inflow }} & =\left(\rho_{\infty}, \rho_{\infty} \vec{v}_{\infty}, \frac{p_{b-1}}{\gamma-1}+\frac{1}{2} \rho_{\infty} \vec{v}_{\infty}^{2}, \rho_{\infty} \check{\nu}_{b-1}\right)^{T} \\
\vec{Q}_{\text {outflow }} & =\left(\rho_{b-1}, \rho_{b-1} \vec{v}_{b-1}, \frac{p_{\infty}}{\gamma-1}+\frac{1}{2} \rho_{b-1} \vec{v}_{b-1}^{2}, \rho_{b-1} \check{\nu}_{b-1}\right)^{T}
\end{aligned}
$$

This boundary condition can only be used accurately for steady flows under the condition that the boundaries are far away from the relevant flow phenomena such that interference with the unphysical extrapolations is minimized. This means that e.g. the calculation of a two-dimensional flow around an airfoil requires a domain of about 50 times chord lengths of the airfoil. 
Simplified characteristics: Starting from the characteristic form of the EULER equations (G.12) the time derivative of $\hat{\vec{C}}$ can be neglected if the variation along the characteristics disappears. The sign of the eigenvalues $\lambda_{i}$ determines the direction of information

$$
\hat{C}_{i}=\left\{\begin{array}{ll}
\hat{C}_{i b-1} & \text { for } \lambda_{i} \geq 0 \\
\hat{C}_{i \infty} & \text { for } \lambda_{i}<0
\end{array} .\right.
$$

$U$ can be positive or negative leading in subsonic flows to inflow or outflow, respectively. Providing the the direction normal to the boundary to be $\xi$ in the following formulation ${ }^{2}$ the conditions for the primitive variables can be derived using equation (G.13) and extrapolating the assumed constant values $\rho_{*}$ and $a_{*}$.

Inflow:

$$
\begin{aligned}
& p_{b}=\frac{1}{2}\left[p_{b-1}+p_{\infty}\right. \\
& \left.+\frac{\rho_{b-1} a_{b-1}}{|\nabla \xi|}\left(\xi_{x}\left(u_{\infty}-u_{b-1}\right)+\xi_{y}\left(v_{\infty}-v_{b-1}\right)+\xi_{z}\left(w_{\infty}-w_{b-1}\right)\right)\right] \\
& \rho_{b}=\rho_{\infty}+\frac{1}{a_{b-1}^{2}}\left(p_{b}-p_{\infty}\right) \quad \check{d}=\frac{\left(p_{b}-p_{\infty}\right)}{\rho_{b-1} a_{b-1}} \\
& u_{b}=\rho_{b}\left(u_{\infty}+\frac{\xi_{x}}{|\nabla \xi|} \check{d}\right) \\
& v_{b}=\rho_{b}\left(v_{\infty}+\frac{\xi_{y}}{|\nabla \xi|} \check{d}\right) \\
& w_{b}=\rho_{b}\left(w_{\infty}+\frac{\xi_{z}}{|\nabla \xi|} \check{d}\right)
\end{aligned}
$$

Outflow (furthermore $U_{b}=U_{\infty}$ is assumed):

$$
\begin{aligned}
& p_{b}=p_{\infty} \\
& \rho_{b}=\rho_{b-1}+\frac{1}{a_{b-1}^{2}}\left(p_{b}-p_{b-1}\right) \quad \check{d}=\frac{\left(p_{b}-p_{b-1}\right)}{\rho_{b-1} a_{b-1}} \\
& u_{b}=\rho_{b}\left(u_{b-1}+\frac{\xi_{x}}{|\nabla \xi|} \check{d}\right) \\
& v_{b}=\rho_{b}\left(v_{b-1}+\frac{\xi_{y}}{|\nabla \xi|} \check{d}\right) \\
& w_{b}=\rho_{b}\left(w_{b-1}+\frac{\xi_{z}}{|\nabla \xi|} \check{d}\right) .
\end{aligned}
$$

The conservative variables are easily calculated as

$$
\vec{Q}_{\text {inflow/outflow }}=\left(\rho_{b}, \rho_{b} \vec{v}_{b}, \frac{p_{b}}{\gamma-1}+\frac{1}{2} \rho_{b} \vec{v}_{b}^{2}, \rho_{b} \check{\nu}_{b-1}\right)^{T} .
$$

This boundary condition has been used successfully in many aerodynamic flows with a steady state solution [25, 26, 27] since it shows better numerical behavior than the simple extrapolation techniques discussed in equations $(4.22,4.23)$.

\footnotetext{
${ }^{2}$ other directions can be derived by exchanging the metric terms
} 
Non-reflecting boundary conditions: A new category of boundary conditions defines the residual Res $(\vec{Q})_{b}$ instead of $\vec{Q}_{b}$ at the boundary. By choosing the values of $\mathcal{L}_{i}$ in equations (G.14) the time derivatives in equations (G.15) can be calculated and integrated using the remaining part of the discretized equations. Several formulations have been proposed for $\mathcal{L}_{i}[79,85,124]$ to minimize or prevent reflections of disturbances at the boundaries. Usually such an accurate formulation of unsteady phenomena is essential in $L E S, D N S$ and computational aeroacoustics $C A A$ since inaccuracies or disturbances near the boundaries reduce the high accuracy of the global numerical scheme and diminish the quality of the solution. The weak-reflecting boundary condition based on the formulation of POINSOT and LELE [79] with pressure relaxation has been implemented for the wake flow problem. Details about the implementation and results can be found in [85]. The velocities at the inflow boundaries $\vec{v}_{\text {inflow }}$ were imposed by simple induction laws using the vorticity within the domain of integration as the driving source. Then, the residual on the boundary can be calculated from the time derivative of the primitive variables. The equation of the turbulence model is unaffected by this procedure and the eddy viscosity is still extrapolated at the boundary $\check{\nu}_{b}=\check{\nu}_{b-1}$.

The aforementioned boundary conditions represent separate formulations for inflow or outflow. In the case of vortical dominated flows like the wake flow of a lifting device there exist boundaries where the flow direction and the exact values of $\vec{v}, p, \rho, T$ are unknown. The generation of large integration domains would reduce the impact of the boundary condition on the solution but also would increase the number of grid points and subsequently the computational effort needed for the solution. Therefore, a new approach is proposed, which can be applied to different formulations of the nonreflecting boundary condition concept. The boundary is treated simultaneously for the inflow and outflow region using the tanh function as blending function. Depending on the angle $\theta$ between

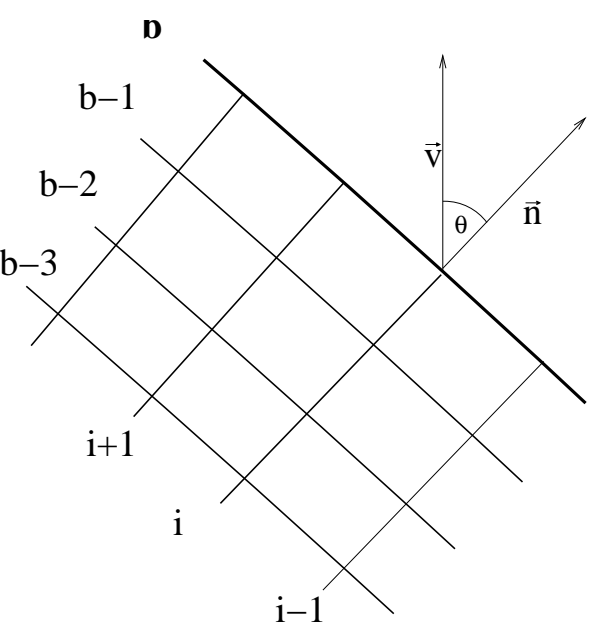

Figure 4.7: Definitions at the boundary the velocity vector $\vec{v}$ and the normal vector $\vec{n}$ of the boundary as shown in figure 4.7 such a formulation allows a smooth transition between the different boundary conditions in regions where the velocity vector is almost tangential to the boundary. The outflow $B C_{b, \text { outflow }}^{n}$ and inflow $B C_{b, \text { inflow }}^{n}$ parts at time level $n$ are multiplied by the factors $f_{\text {outflow }}$ and $f_{\text {inflow }}$, respectively, and then added

$$
\begin{aligned}
f_{\text {outflow }} & =\tanh \left((\pi-2.0 \cdot \theta) \cdot a_{B C}\right) \cdot 0.5+0.5 \text { with } a_{B C} \sim \mathcal{O}(10) \\
f_{\text {inflow }} & =1.0-f_{\text {outflow }} \\
B C_{b}^{n} & =f_{\text {outflow }} \cdot B C_{b, \text { outflow }}^{n}+f_{\text {inflow }} \cdot B C_{b, \text { inflow }}^{n} .
\end{aligned}
$$

Furthermore, a correction of the residuals on the boundaries using the exact values from the interior of the computational domain is used. Beginning with an initial distribution 
on the domain $\vec{Q}_{i n i t}$, which fulfills the governing equations, the calculation in the first time step is based on correct values. Therefore, all the residuals $\operatorname{Res}(\vec{Q})$ are correct except for the values on the boundary $\operatorname{Res}(\vec{Q})_{b}=B C_{b}$. They have to be determined e.g. by the aforementioned non-reflecting boundary condition. The boundary condition can be calculated not only on the boundary $b$ but also, for example, on the first inner line $b-1$ within the computational domain. Then, the residuals on the first inner line $\operatorname{Res}(\vec{Q})_{b-1}$ and those calculated by the boundary condition $B C_{b-1}$ are known. Using the information at $b-1$ on the deviation of the boundary condition from the exact solution the boundary residuals can be finally corrected the following extrapolation from the interior

$$
\begin{aligned}
B C_{b-1, \text { correction }}^{n} & =\operatorname{Res}_{b-1}^{n}-B C_{b-1}^{n} \\
\operatorname{Res}(\vec{Q})_{b, \text { correction }}^{n} & =B C_{b}+B C_{b-1, \text { correction }}^{n} .
\end{aligned}
$$

This methodology theoretically contradicts to a certain extent the propagation of information since the information is propagated from the inside of the domain to the boundary and can therefore lead to an ill-posed problem. On the other hand, this correction is at least one order of magnitude smaller than the values on the boundary determined by the boundary condition itself.
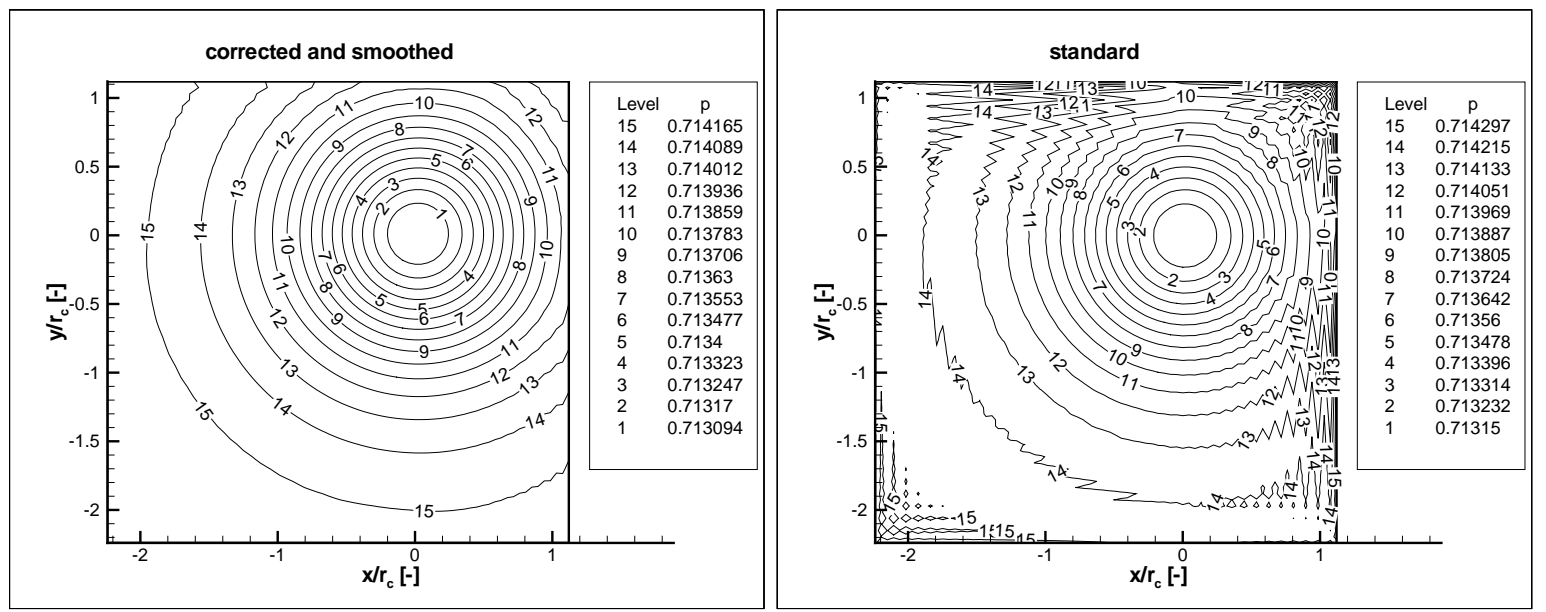

Figure 4.8: Pressure distribution of a slightly compressible LAMB-OSEEN vortex after 15000 sub iterations using modified (left) and standard (right) boundary condition

In figure 4.8 a solution of a slightly compressible LAMB-OSEEN vortex, which is described in chapter 5.1.2, located near the upper right corner of the integration domain in the origin of the coordinate system using the aforementioned boundary condition is compared with that of an uncorrected boundary condition. The sensitive pressure distribution, which is in the range of $\mathcal{O}\left(10^{-4}\right)$, shows that the correction leads to a much smoother circular distribution and suppresses instabilities. In other words, although the new formulation is computationally more expensive and is theoretically not rigorously analyzed its superiority compared to the old approximation shows that it makes sense to apply the residual based approximation. 


\subsubsection{Boundary Conditions for the Engine Jet}

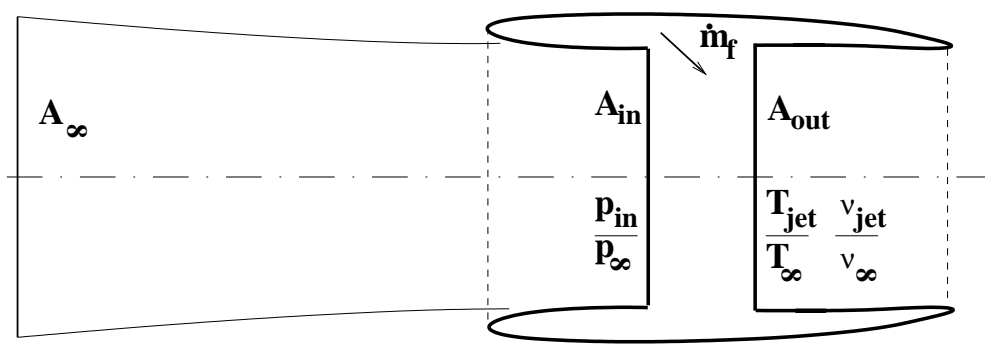

Figure 4.9: Boundary condition for engine jet simulation

The engine jet is simulated through a reformulated version of the boundary conditions proposed by RUDNIK et al. in [94]. The inflow and outflow surfaces $A_{\text {in }}, A_{\text {out }}$ and the surface of an inlet streamtube at infinity $A_{\infty}$ under freestream conditions are sketched in figure 4.9. The engine inflow condition is given by the non-dimensional inlet mass flow $\dot{m}_{i n}$ expressed by the ratio $\varepsilon_{\infty / \text { in }}$ and using the one-dimensional isentropic relations

$$
\begin{aligned}
\varepsilon_{\infty / i n} & =\frac{A_{\infty}}{A_{\text {in }}}=\frac{\dot{m}_{i n}}{A_{\text {in }} M a_{\infty} \sqrt{\gamma p_{\infty} \rho_{\infty}}} \\
& =\frac{A_{\infty}}{A^{\text {critic }}} \frac{A^{\text {critic }}}{A_{\text {in }}}=\frac{M a_{i n}}{M a_{\infty}}\left[\frac{1+\frac{\gamma-1}{2} M a_{\infty}^{2}}{1+\frac{\gamma-1}{2} M a_{i n}^{2}}\right]^{\frac{\gamma+1}{2(\gamma-1)}}
\end{aligned}
$$

Given the freestream values the MACH number at engine inflow $M a_{\text {in }}$ can be calculated iteratively. The pressure ratio at engine inflow can then be calculated according to

$$
\frac{p_{i n}}{p_{\infty}}=\frac{p_{i n}}{p_{0}} \frac{p_{0}}{p_{\infty}}=\left[\frac{1+\frac{\gamma-1}{2} M a_{\infty}^{2}}{1+\frac{\gamma-1}{2} M a_{i n}^{2}}\right]^{\frac{\gamma}{\gamma-1}} .
$$

The engine inflow boundary condition can be formulated according to a regular outflow boundary condition like in equations $(4.23,4.26)$. The mass flow $\dot{m}_{\text {in }}$ is adjusted automatically as a consequence of the imposed pressure $p_{i n}$, the value of which is determined via equation (4.32). The actual $\dot{m}_{i n}$ is calculated by

$$
\dot{m}_{i n}=\int_{A_{i n}} \rho_{i n} \vec{v} \vec{n} d A
$$

The extrapolated pressure and the temperature ratios $\frac{p_{b-1}}{p_{\infty}}, \frac{T_{j e t}}{T_{\infty}}$ at the engine exhaust are used to calculate the density $\rho_{\text {out }}=\rho_{\infty} \frac{p_{b-1}}{p_{\infty}} \frac{T_{\infty}}{T_{j e t}}$ which is used to determine a constant jet velocity at the outflow plane $\vec{v}_{\text {out }}$ normal to $A_{\text {out }}$

$$
\left|\vec{v}_{\text {out }}\right|=\frac{\dot{m}_{\text {in }}\left(1+\dot{m}_{f} / \dot{m}_{\text {in }}\right)}{A_{\text {out }} \rho_{\text {out }}}
$$


where $\dot{m}_{f}$ represents an additional mass flow, e.g. fuel, that is injected into the flow. The engine outflow boundary condition can be formulated according to a regular inflow boundary condition like in equations $(4.22,4.25)$ with the turbulent viscosity $\check{\nu}_{\text {out }}$ imposed explicitly according to the ratio $\check{\nu}_{\text {jet }} / \check{\nu}_{\infty}$. Unlike the boundary condition described in [94] this boundary condition couples inflow and outflow within the engine and as such guarantees mass conservation. 


\section{Chapter 5}

\section{Results and Discussion}

"The rolling up of the trailing vortices associated with high-aspect-ratio wings is of little practical importance ..."

Spreiter 6 Sacks, Journal of Aeronautical Sciences, 1951.

The FAVRE-averaged NAVIER-STOKES equations described in chapter 2 and the turbulence models given in chapter 3 are solved according to the numerical solution algorithm in chapter 4 for several flow problems.

In the first part results achieved with the proposed new turbulence model are compared with experimental results and with data of other turbulence models especially the widely used SpalarT-Allmaras model. The emphasis is put on the validation of the proposed turbulence model and the numerical methods used. In the second part the flow around a rectangular wing including an engine jet and the wake is discussed in detail with focus on the interaction of the wingtip vortex and the engine jet in the near field.

\section{$5.1 \quad$ Validation}

The thorough validation of the numerical scheme and the turbulence model via fundamental flow problems is essential for accurate numerical predictions of technically relevant flows. A comparison of the calculations with measurements or in some cases also analytical asymptotic solutions is a measure for the quality and reliability of the numerical methods and its implementation. The flow around the wing including the engine jet and the wake can be subdivided into three relevant flow categories. The free shear flow, the vortical wake and the wall boundary layer. For each of these categories several validating flow simulations will be presented and discussed. 


\subsubsection{Turbulent Free Shear Flows}

The free shear flows presented in figure 5.1 represent fundamental flows that can be described by the boundary layer theory. They have been investigated theoretically, experimentally and numerically in many works, for an overview see .e.g. [31, 106, 139]. The existence of self-similar solutions of the turbulent free shear flows simplifies the theoretical and numerical analysis considerably. A similarity variable $\eta=y / x$ can be used to transform the equations of motion for incompressible steady flows. The transformation leads to one single ordinary differential equation for the momentum and one or two equations for the turbulence model depending on the number of equations used by the turbulence model. Mass conservation is also automatically satisfied. All the equations depend only on the one similarity coordinate $\eta$. A detailed derivation of the similarity equations can be found in [139].

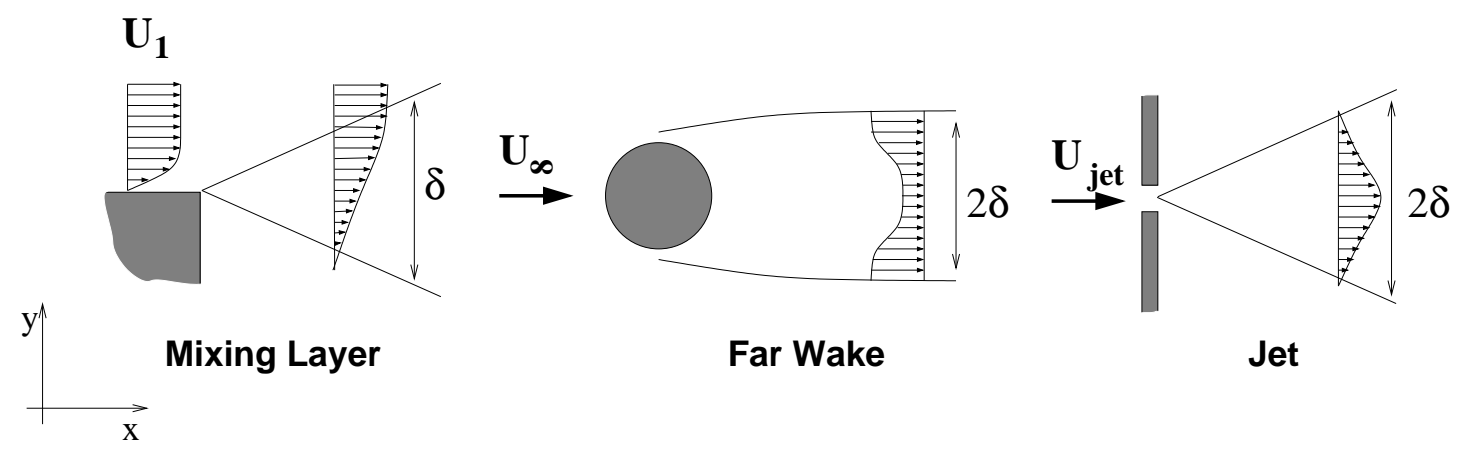

Figure 5.1: Free shear flows

Simulations for different turbulent free shear layers were performed using the $k-\omega$, the SpalarT-Allmaras and the new turbulence model derived in chapter 3.1.2. Measurements of these flows are also presented for comparison.

The mixing layer the velocity distributions of which are depicted in figure 5.2 represents the basic shear flow. All three turbulence models give likewise accurate predictions of the flow.

The far wake flow characterized by a velocity deficit and encountered in aerodynamical flows behind the trailing edge of wings is presented in figure 5.3. The three models give similar results for the core region but deviate at the edge of the shear layer. The $k-\omega$ model shows a much smoother approach of the velocity profile at the edge than the Spalart-Allmaras model. Both models under- or overpredict the velocity deficit approaching the edge, whereas the new model give the best result in the outer region compared to the measurements in [24] and [136]. 


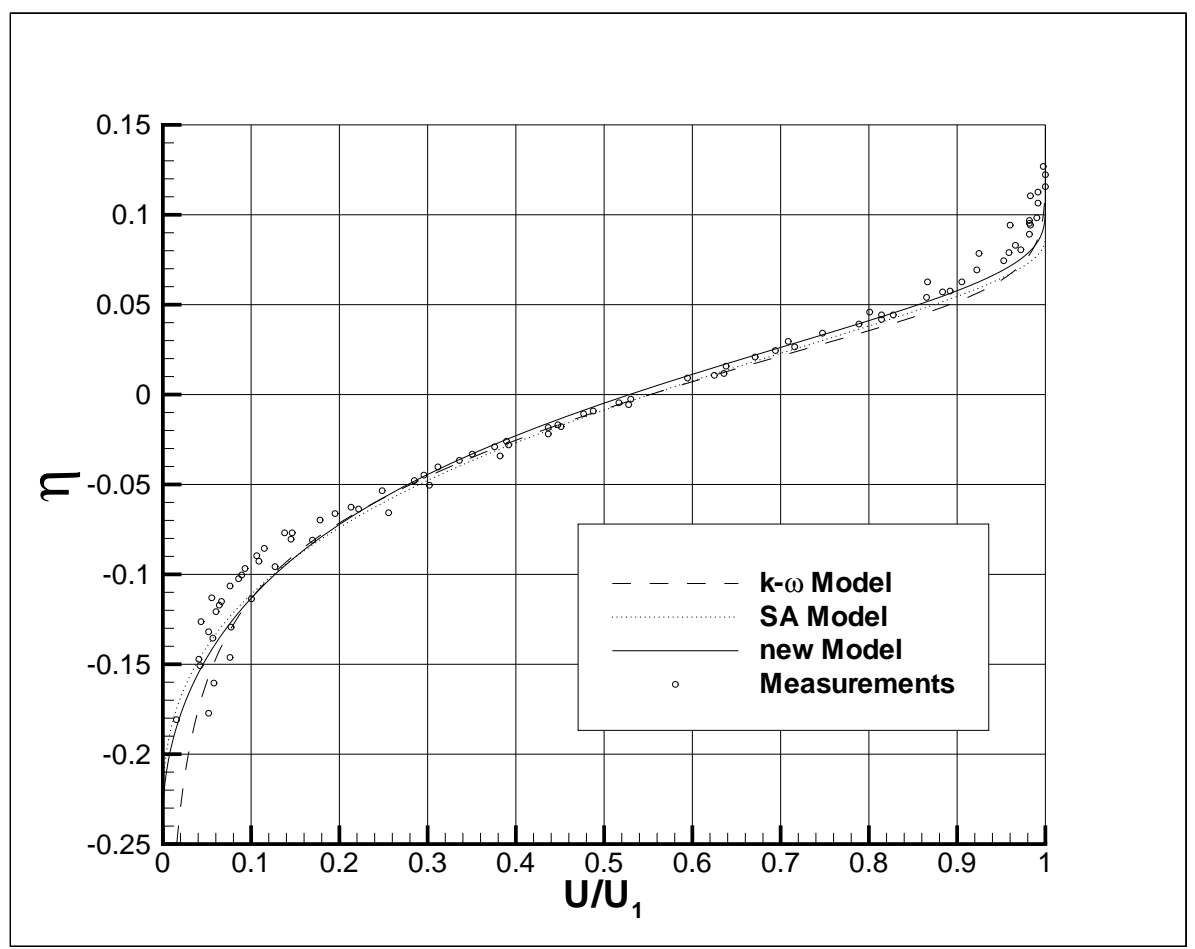

Figure 5.2: Comparison of computed and measured velocity profiles for the mixing layer. Measurements by LiePMann and LaUfer [57]

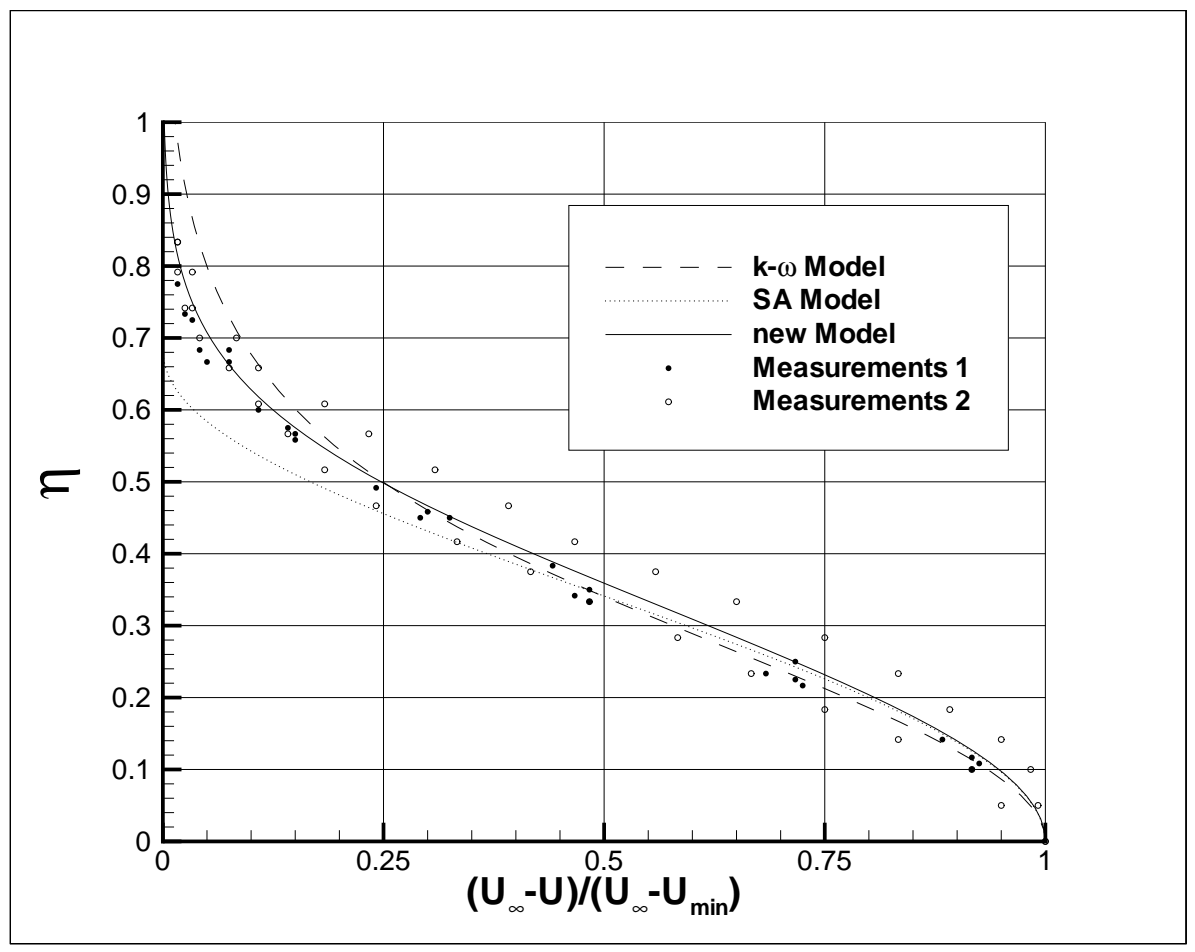

Figure 5.3: Comparison of computed and measured velocity profiles for the far wake. Measurements (1) by Fage and Falkner [24], measurements (2) by Weygandt and Mehta [136] 


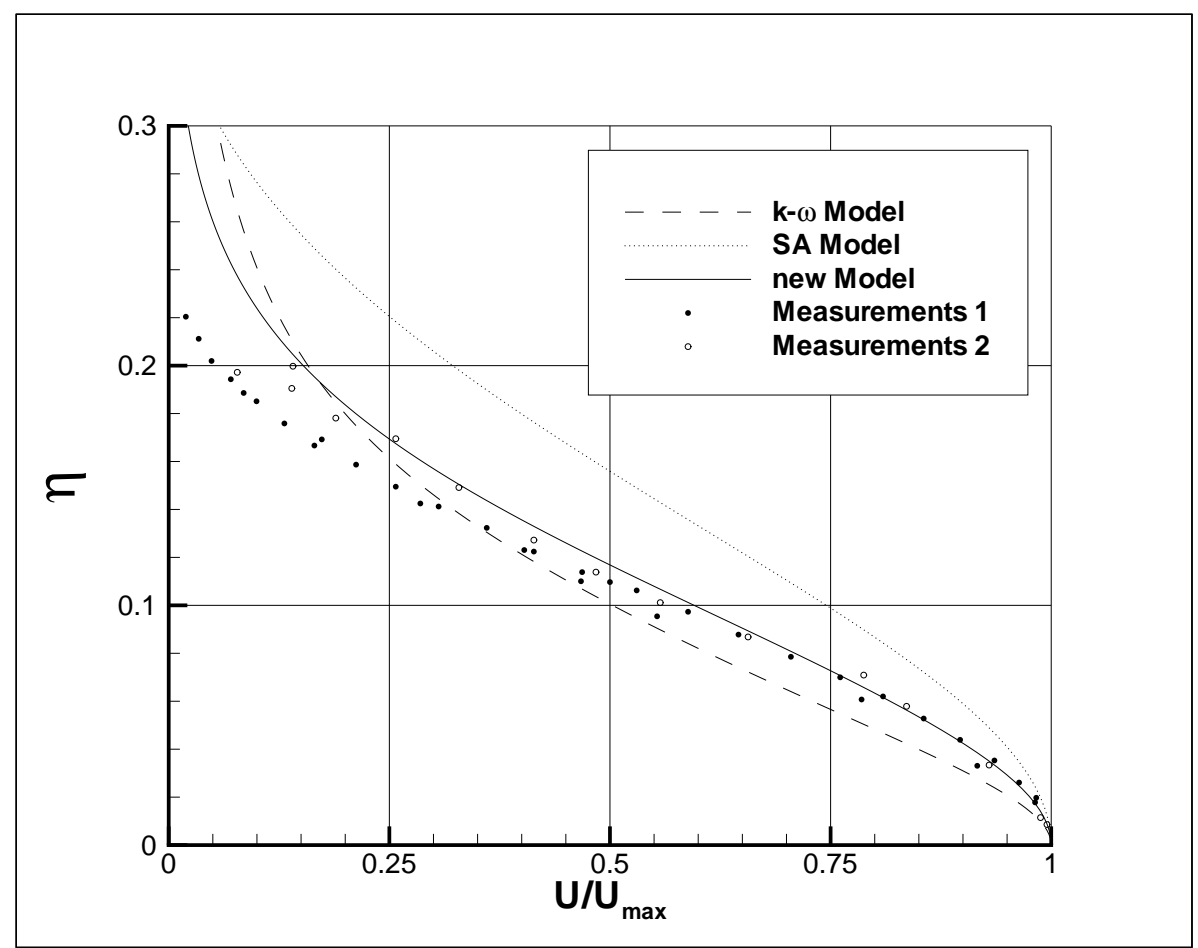

Figure 5.4: Comparison of computed and measured velocity profiles for the plane jet. Measurements (1) by Badbury [7], measurements (2) by Heskestad [39]

Next, three types of jets are simulated. The plane jet is shown in figure 5.4 and the round axisymmetric jet is given in figure 5.5. Furthermore, a comparison of the numerical results of the radial jet flow occurring when two jets of equal strength collide and spread radially is presented in figure 5.6. The SPALART-Allmaras model performs poorly in any case. This is consistent with the discussion in chapter 3.1.1 pointing out that this model has been optimized for aerodynamic applications which include wake flows and mixing layers but jet flows were not considered. The missing destruction term of the original SPALART-ALLMARAS model far away from the wall is responsible for this behavior. The new model gives the best prediction in the core and the edge region of the plane jet and the quality of the findings is comparable with the $k$ - $\omega$ model in the middle region.

The results for the round jet depicted in figure 5.5 show a dramatic discrepancy of the distribution of the SPALART-AllmaRAS model. In a wide range it overpredicts the velocities and the spreading rate by a factor greater than 2 . The $k-\omega$ and the new model yield almost equally good velocity distributions. However, especially in the outer region the comparison with the experimental data evidences a slight superiority of the new one-equation model over the $k$ - $\omega$ formulation.

The comparison of numerical results for the radial jet presented in figure 5.6 also confirm the analogous behavior of the $k-\omega$ and the new model with a somewhat smaller spreading for the new one-equation formulation. 


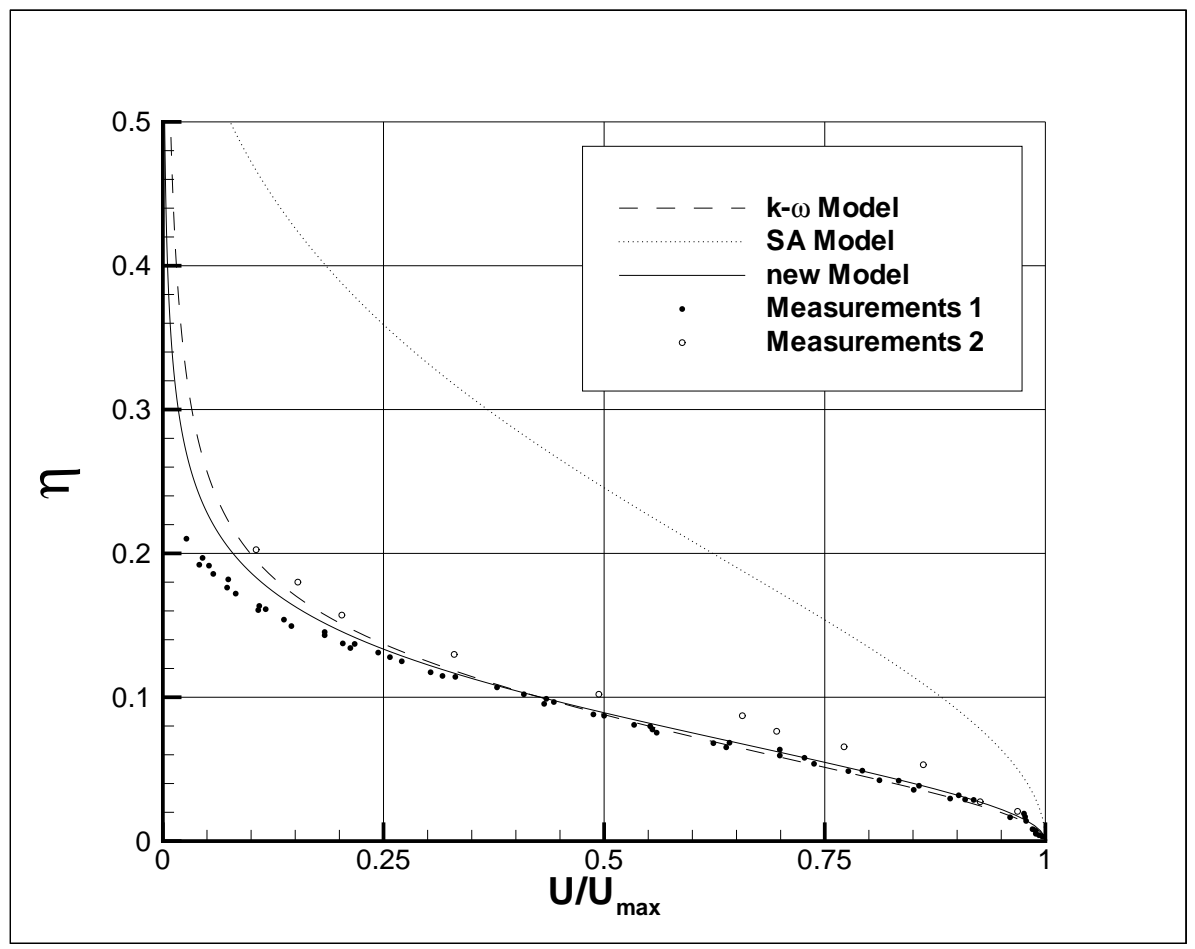

Figure 5.5: Comparison of computed and measured velocity profiles for the round jet. Measurements (1) by Wygnanski And Fiedler [140], measurements (2) by Rodi [88]

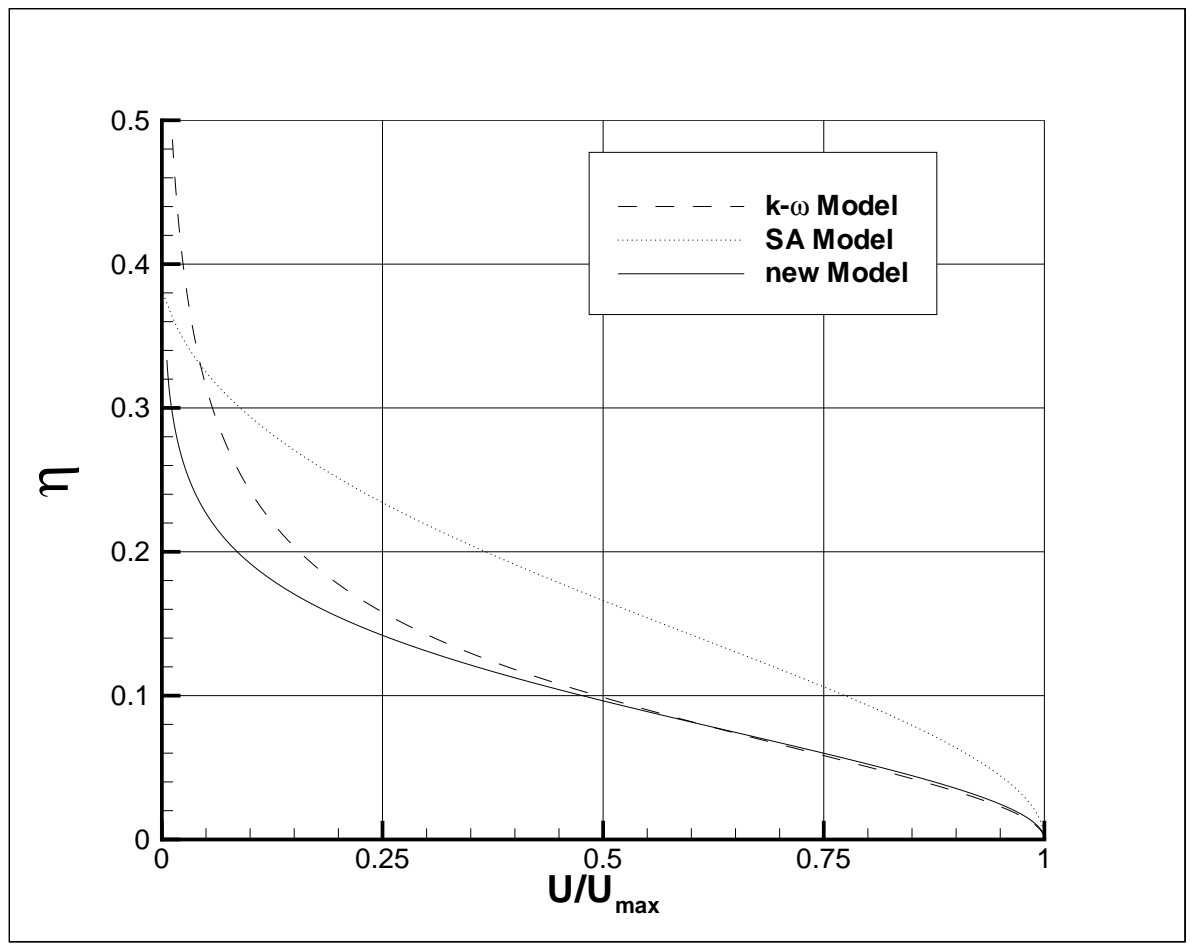

Figure 5.6: Comparison of computed and measured velocity profiles for the radial jet 
The spreading rates of the described shear flows are given in table 5.1. The spreading rate is generally defined as the value of the similarity variable $\eta$ where the velocity or velocity deficit is half its maximum value. For the mixing layer the spreading rate is defined as the difference between the values of $\eta$ where $U^{2} / U_{1}^{2}$ is 0.9 and 0.1 .

\begin{tabular}{|l|c|c|c|c|c|}
\hline Flow & $k-\omega$ Model & $S A$ Model & new Model & Measured & Experiment \\
\hline \hline Mixing Layer & 0.105 & 0.108 & 0.109 & 0.155 & {$[57]$} \\
\hline Far Wake & 0.340 & 0.341 & 0.359 & 0.365 & {$[24]$} \\
\hline Plane Jet & 0.101 & 0.156 & 0.117 & $0.100-0.110$ & {$[88,140]$} \\
\hline Round Jet & 0.088 & 0.246 & 0.089 & $0.086-0.096$ & {$[7,39]$} \\
\hline Radial Jet & 0.099 & 0.166 & 0.096 & $0.096-0.110$ & taken from $[139]$ \\
\hline
\end{tabular}

Table 5.1: $\quad$ Flow spreading rates for turbulent free shear flows

The table provides a concise criterion of the predictive capabilities of the turbulence models for free shear layers and confirms the quality of the new model. The best agreement with the experimental findings for the spreading rates of the mixing layer and the wake is achieved by the new model. Furthermore, almost all popular turbulence models predict a stronger spreading of the round jet than the plane jet, which contradicts the experimental results given in table 5.1. This phenomenon is known as the round/plane jet anomaly and is discussed at length in [80, 139]. The $k$ - $\omega$ model and the new one-equation model represent the exceptions, i.e. they yield the proper tendency as to the spreading rate for both jet flows.

\subsubsection{Isolated Vortex}

Even though the vortex generation and vortex dynamics play a major role in almost all realistic flow situations especially in the wake of a lifting device like the wing no standard test cases exist for such flows. Slender trailing vortices in the wake usually persist for a long distance and can be described in general by inertial laws like the Biot-SAVART law [96]. Consequently, viscous effects are neglected and as such physical aspects of the vortex dynamics like e.g. decay are not captured. There are, however, several theoretical results for a simplified line vortex in laminar and turbulent flows that are discussed in detail in the next sections.

\section{Vortex in Laminar Flow}

An analytic solution can be obtained for a two-dimensional circular symmetric temporally developing viscous LAMB-OSEEN vortex $[4,96]$. Depending on the initial distribution of the vortex at a time level $t=t_{\text {init }}$ with the circulation $\Gamma_{\text {init }}$ and the molecular viscosity $\nu$ the maximum tangential velocity $v_{1}$ and the core radius $r_{1}$, which is defined by the location of the maximum velocity, read

$$
\Gamma=\Gamma_{\text {init }}\left(1-e^{-\frac{r^{2}}{4 \nu t}}\right) \quad r_{1} \approx 2.24 \sqrt{\nu t} \quad v_{1}=\frac{\Gamma}{2 \pi r_{1}} .
$$


Different reconstruction schemes of the numerical discretization of the advective terms as described in chapter 4.2.1 are compared for the temporal development of $r_{1}$ and $v_{1}$ in figures 5.7 and 5.8. The location of the maximum velocity as well as the position of the core radius were determined without interpolation on the grid. The size of the grid cell is visualized by the error bars. The boundaries of the integration domain were chosen 30 core radii away from the vortex center to minimize the influence of the boundary condition during the temporal evolution of the vortex. The presentations
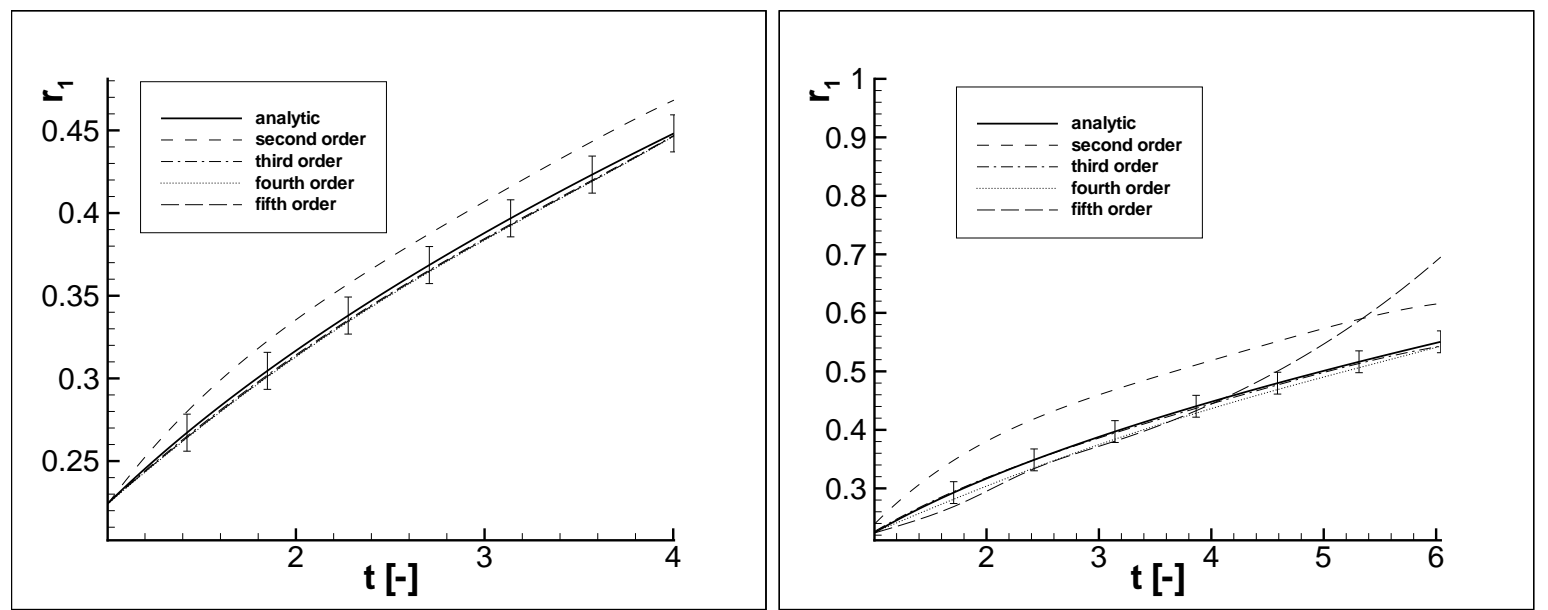

Figure 5.7: Temporal development of the core radius $r_{1}$ for 5 (left) and 3 (right) grid points per core radius using different reconstruction schemes
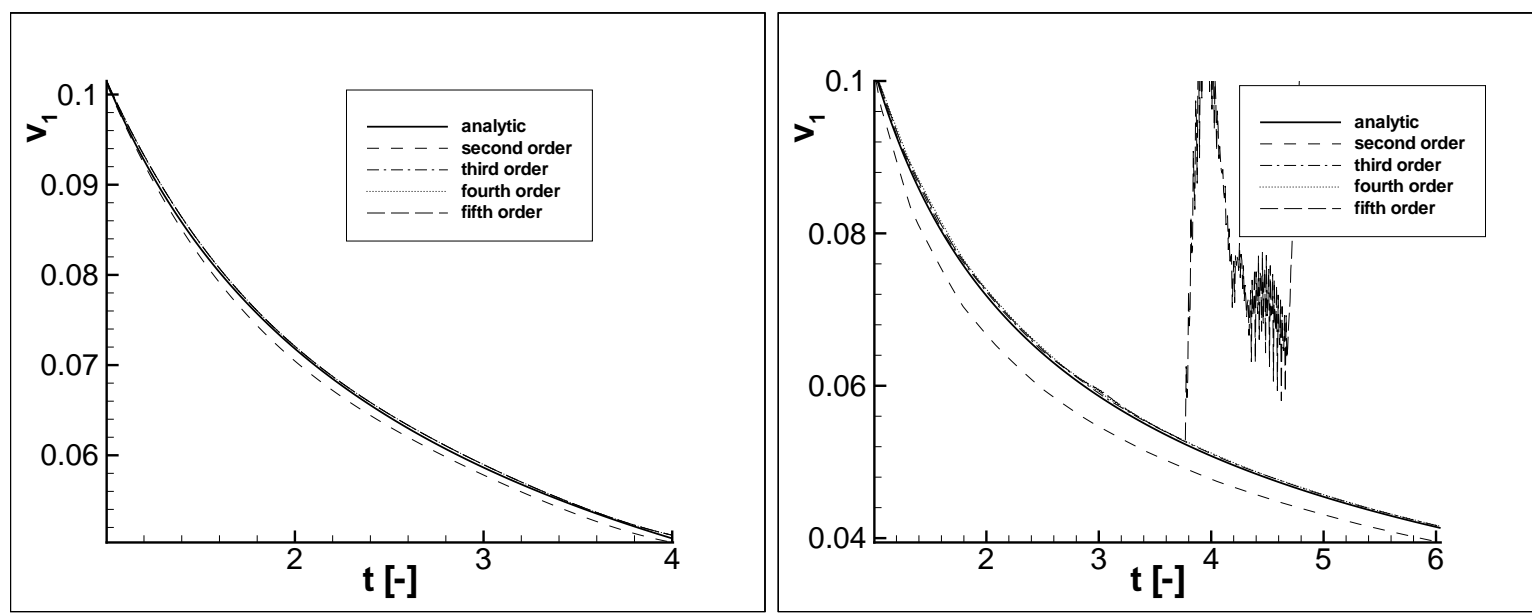

Figure 5.8: Temporal development of the maximum tangential velocity $v_{1}$ for 5 (left) and 3 (right) grid points per core radius using different reconstruction schemes

show the results for very coarse resolutions of 3 and 5 grid points per core radius. Unlike the third-, fourth- and fifth-order schemes the second-order scheme substantially deviates in both cases from the analytical solution. The slight improvements for the fourth- and fifth-order accurate reconstruction schemes over the third-order accurate scheme are offset by the smaller $C F L$ condition of the higher-order schemes. For the fourth-order accurate scheme the time step had to reduced to one half of the 
value for the the second- and third-order schemes and the fifth-order accurate scheme showed an unstable behavior on the coarser grid for time levels $t>3.8$ as depicted in figure 5.8. Therefore, it can be concluded that the third-order accurate reconstruction scheme is the best compromise between accuracy and stability for the aforementioned calculations.

\section{Vortex in Turbulent Flow}

The behavior of a longitudinal axisymmetric slender vortex in turbulent flow represents a simplified example of the wingtip vortex. Assuming that the mean properties of the flow are independent of the axial coordinate the incompressible REYNOLDS averaged equations in polar coordinates read for the azimuthal velocity $v_{t}$ and the radial velocity $u_{r}$ in the radial coordinate $r$

$$
\frac{\partial \bar{v}_{t}}{\partial t}=\frac{1}{\rho}\left[\frac{1}{r^{2}} \frac{\partial\left(r^{2} \bar{\tau}\right)}{\partial r}-\frac{1}{r^{2}} \frac{\partial}{\partial r} \overline{r^{2} v_{t}^{\prime} u_{r}^{\prime}}\right]
$$

with

$$
\bar{\tau}=\mu r \frac{\partial}{\partial r}\left(\frac{\bar{v}_{t}}{r}\right)
$$

The REYNOLDs stress is modeled according to the BoussinesQ hypothesis

$$
\overline{v_{t}^{\prime} u_{r}^{\prime}}=\mu_{t} r \frac{\partial}{\partial r}\left(\frac{\bar{v}_{t}}{r}\right)
$$

which leads to the following tangential momentum equation

$$
\frac{\partial \bar{v}_{t}}{\partial t}=\left(\nu+\nu_{t}\right)\left(\frac{\partial^{2} \bar{v}_{t}}{\partial r^{2}}+\frac{1}{r} \frac{\partial \bar{v}_{t}}{\partial r}-\frac{\bar{v}_{t}}{r^{2}}\right)+\left(\frac{\partial \bar{v}_{t}}{\partial r}-\frac{\bar{v}_{t}}{r}\right) \frac{\partial \nu_{t}}{\partial r} .
$$

In terms of the circulation $\Gamma=2 \pi r \bar{v}_{t}$ equation (5.5) gives

$$
\frac{\partial \Gamma}{\partial t}=\left(\nu+\nu_{t}\right)\left(\frac{\partial^{2} \Gamma}{\partial r^{2}}-\frac{1}{r} \frac{\partial \Gamma}{\partial r}\right)+\left(\frac{\partial \Gamma}{\partial r}-2 \frac{\Gamma}{r}\right) \frac{\partial \nu_{t}}{\partial r}
$$

Experimental [42, 75], theoretical [35, 74, 96] and LES [117] as well as DNS [73, 81] data show that self-similarity exists with the similarity variable $\eta=r / r_{1}$ and $r_{1} \sim t^{1 / 2}$ where the subscript 1 denotes the position of maximum tangential velocity. It was first proposed by HOFFMAN and JOUBERT [42] that a triple layer structure of the turbulent vortex core exists obeying different laws for the ratio of circulation $\Gamma / \Gamma_{1}$ :

$$
\begin{array}{rcl}
\text { viscous layer: } & 0 \leq \eta<1 & \frac{\Gamma}{\Gamma_{1}}=C_{v} \eta^{2} \\
\text { logarithmic layer: } & \eta \approx 1 & \frac{\Gamma}{\Gamma_{1}}=1+\ln \eta \\
\text { outer layer: } & \eta \gg 1 & \frac{\Gamma}{\Gamma_{1}}=\frac{\Gamma_{\infty}}{\Gamma_{1}}=\text { const. }>1
\end{array}
$$


with the constant $C_{v}=1.83$ determined experimentally ${ }^{1}$. Furthermore, it was shown [35, 95] that the integral value of circulation deficit yields

$$
\int_{0}^{\infty}\left(1-\frac{\Gamma}{\Gamma_{\infty}}\right) \eta d \eta \sim \frac{2 \nu}{\Gamma_{\infty}} \rightarrow 0 \text { for } \frac{\nu}{\Gamma_{\infty}} \rightarrow 0
$$

at high REynOLDs numbers. Given the similarity laws of the turbulent vortex in equations (5.7-5.9) and the ratio $\frac{\Gamma}{\Gamma_{\infty}}<1.0$ for the viscous layer it can be deduced that there must be a region where $\frac{\Gamma}{\Gamma_{\infty}}>1.0$ to fulfill the aforementioned integral constraint. This means that an overshoot in circulation must exist. The overshoot is a consequence of the self-similar turbulent vortex decaying faster than the laminar LAMB-OSEEN vortex as discussed in [96]. DNS simulations [73, 81] confirm that an overshoot of approximately $2-3 \%$ is present in a turbulent vortex. Calculations by ZEMAN [141] using the $k-\varepsilon$ turbulence model and the REYNOLDS stress closure model of DURBIN [18] show that the correct decay and hence, the correct slight overshoot can only be predicted by the complex REYNOLDS stress model, whereas the standard $k-\varepsilon$ model give overshoots of more than $50 \%$. Similar results were also found by GrASSO et al. [36] who investigated the compressibility effects on the behavior of the turbulent vortex.

Equation (5.5) or (5.6) can be integrated numerically using the analytic solution of the laminar LAMB-OSEEN vortex described in chapter 5.1.2 as initial distribution. The numerical solution using the SPALART-ALlmaRAs Model in its original version plus the rotation and curvature corrections described in equation (3.12) as well as the new turbulence model are compared with the similarity solutions in figure 5.9. All solutions generated for the REYNOLDS number $R e_{\Gamma}=\Gamma_{i n i t} / \nu=1000$ at the dimensionless time $T=t v_{1_{\text {init }}} / r_{1_{\text {init }}}=1000$ give good agreement in the viscous and logarithmic region, whereas the circulation overshoot presented in figure 5.10 deviates strongly depending on the turbulence model used. The new model gives an overshoot of $2 \%$ which is in good agreement with DNS simulations. The SPALART-ALLMARAS model give a much stronger overshoot of more than $20 \%$, a value which is almost unaffected by the rotation correction term. The temporal development of the maximum tangential velocity $v_{1}$ presented in figure 5.11 shows a much stronger decay of the vortex for both SPALARTAllmaras model versions than for the new turbulence model. The slope of the decay $t^{-1 / 2}$ for the self-similar turbulent vortex discussed in $[36,96,141]$ is nearly predicted by the new model and the SPALART-AlLmaRAs model using the rotation and curvature corrections. However, the Spalart-Allmaras model approaches the slope at much smaller values of the maximum tangential velocity than the newly developed model. It can be concluded that the new model gives the best prediction of the circulation overshoot and the decay of the turbulent vortex. The findings of the new model are in excellent agreement with calculations performed using complex REYNOLDS stress models [141] and DNS [73, 81].

\footnotetext{
${ }^{1}$ Note that this behavior is similar to the multi-layer structure of a turbulent boundary layer described in chapter 5.1.3
} 


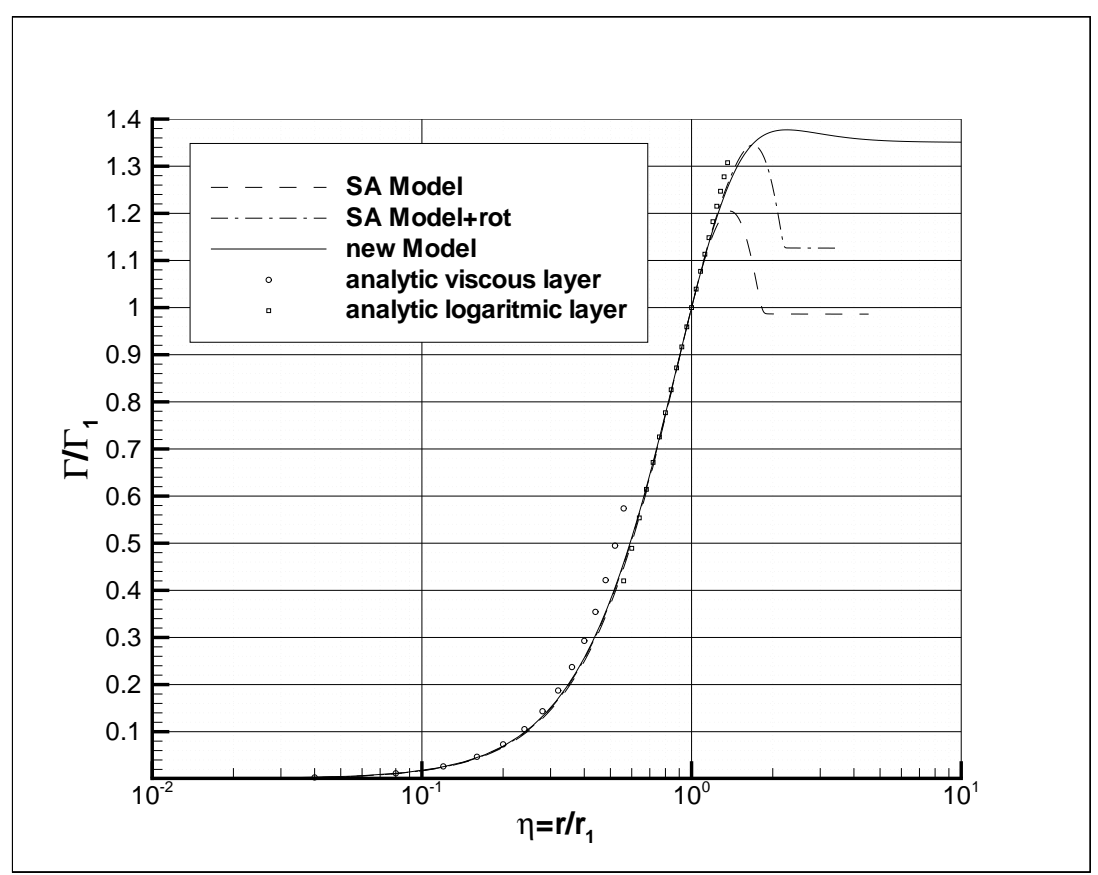

Figure 5.9: Comparison of numerical and analytical solutions of the self-similar turbulent vortex for $R e_{\Gamma}=1000$ at $T=1000$ using different turbulence models

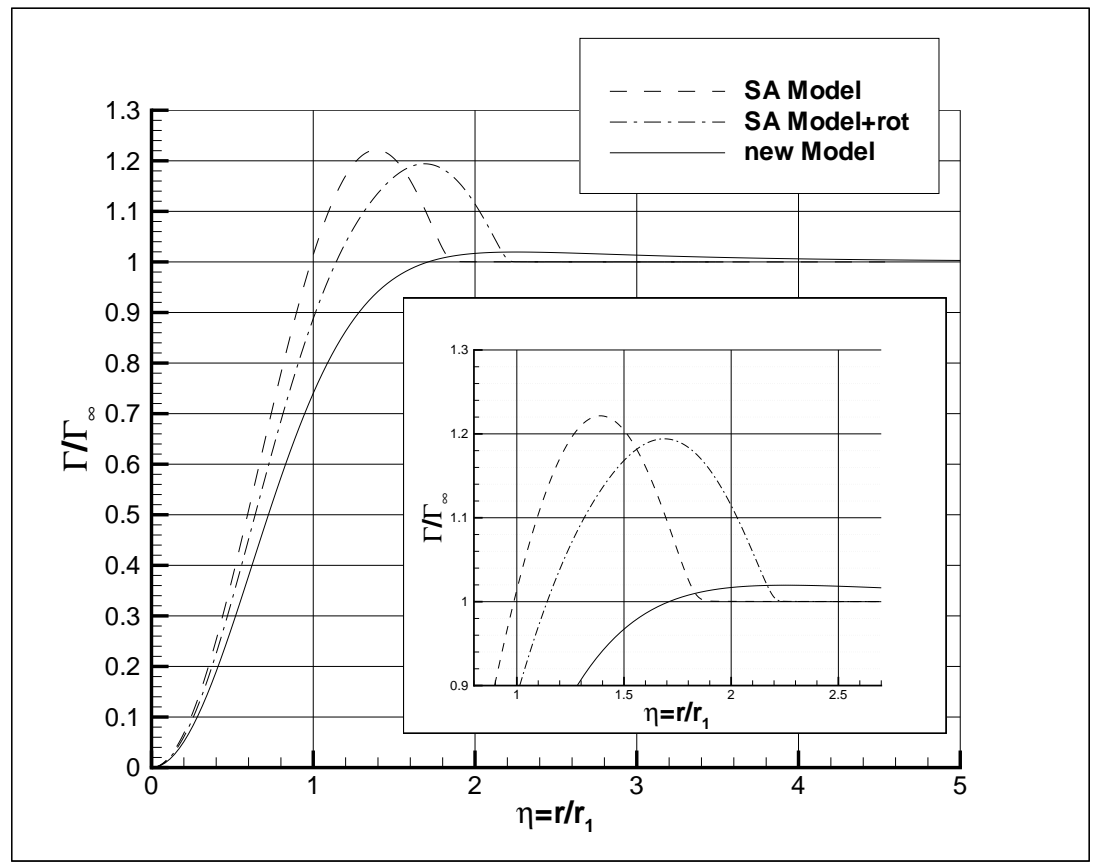

Figure 5.10: Comparison of the distribution of $\Gamma / \Gamma_{\infty}$ for $R e_{\Gamma}=1000$ at $T=1000$ evidencing the circulation overshoot using different turbulence models 


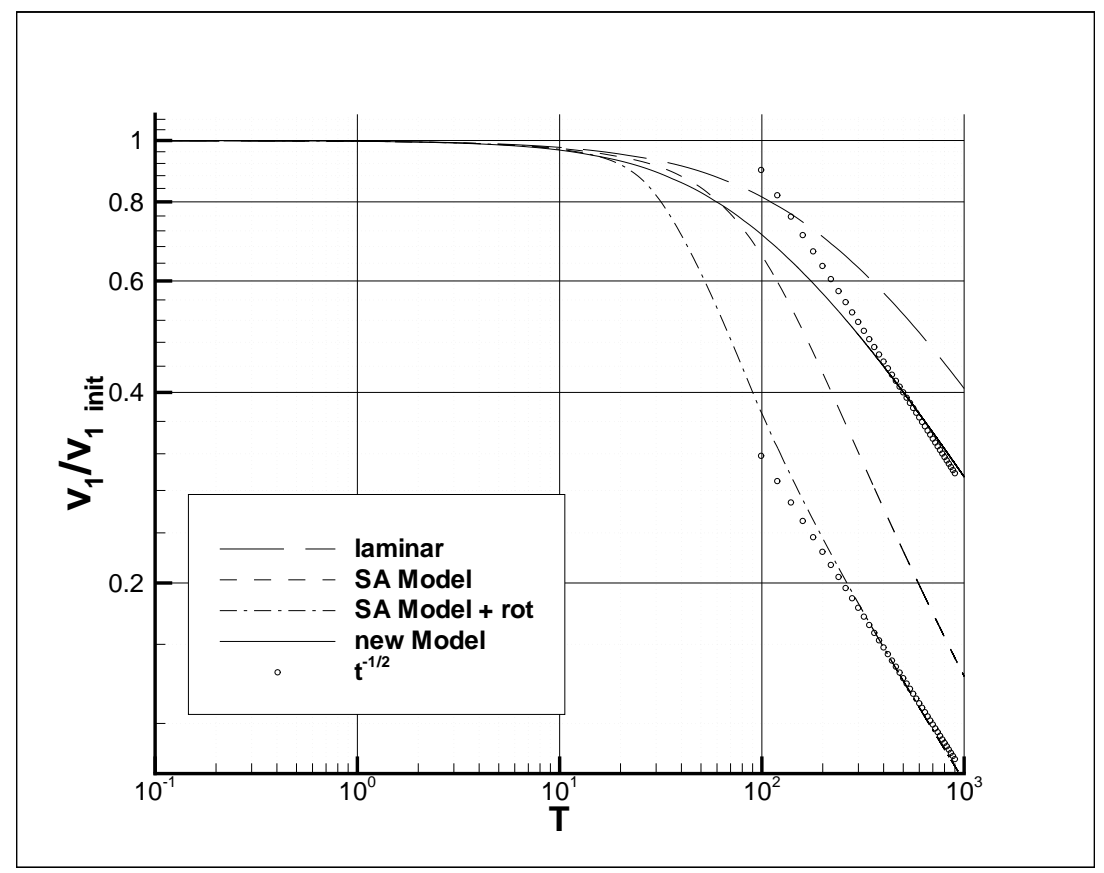

Figure 5.11: Comparison of the temporal development of the maximum tangential velocity $v_{1}$ for $R e_{\Gamma}=1000$ using different turbulence models compared with the self-similar decay which is proportional to $\sim t^{-1 / 2}$

\subsubsection{Flat Plate Boundary Layer}

The flow over a flat plate developing a boundary layer without pressure gradient represents the most fundamental wall boundary layer flow. There exist analytical solutions to both the laminar and the turbulent boundary layer. Both types of flows are encountered during simulations of wall bounded flows with a transition region.

\section{Laminar Flow}

The mathematical description of the flow over a flat plate without a pressure gradient can be reduced to an ordinary differential equations through a series of simplifications and assumptions [106]. The first analytical solution of this problem was given by Blasius [5] for incompressible flow through a series expansion. Using the Blasius solution, the skin-friction coefficient $c_{f}$ can be calculated along the plate according to $[106]$

$$
c_{f}=\frac{0.664}{\sqrt{R e_{x}}} .
$$

If additional assumptions about the temperature and the fluid properties are made solutions of the compressible boundary layer can also be achieved [106, 119]. The selfsimilar solution and numerical calculations of a laminar compressible flow at $M a_{\infty}=$ 0.3 and $R e_{L}=10.0^{5}$ are presented in figure 5.12 as a function of the self-similarity variable $\eta=y / x \sqrt{R e}$. The calculations were performed on a $350 \times 80$ nonuniform 
cartesian grid with a constant temperature at the wall $T_{\text {wall }}=T_{\infty}$. The results show an excellent correlation with theory. This is evidenced by the solution of the velocity component $v$ in the direction normal to the wall, which is some orders of magnitude smaller than the $u$ component in the main flow direction. The skin-friction distribution $c_{f}$ is also well predicted except at the leading edge region where the similarity solution does not hold and at the outflow region at the end of the flat plate where the simple extrapolation boundary condition is also not exactly valid in the boundary layer.
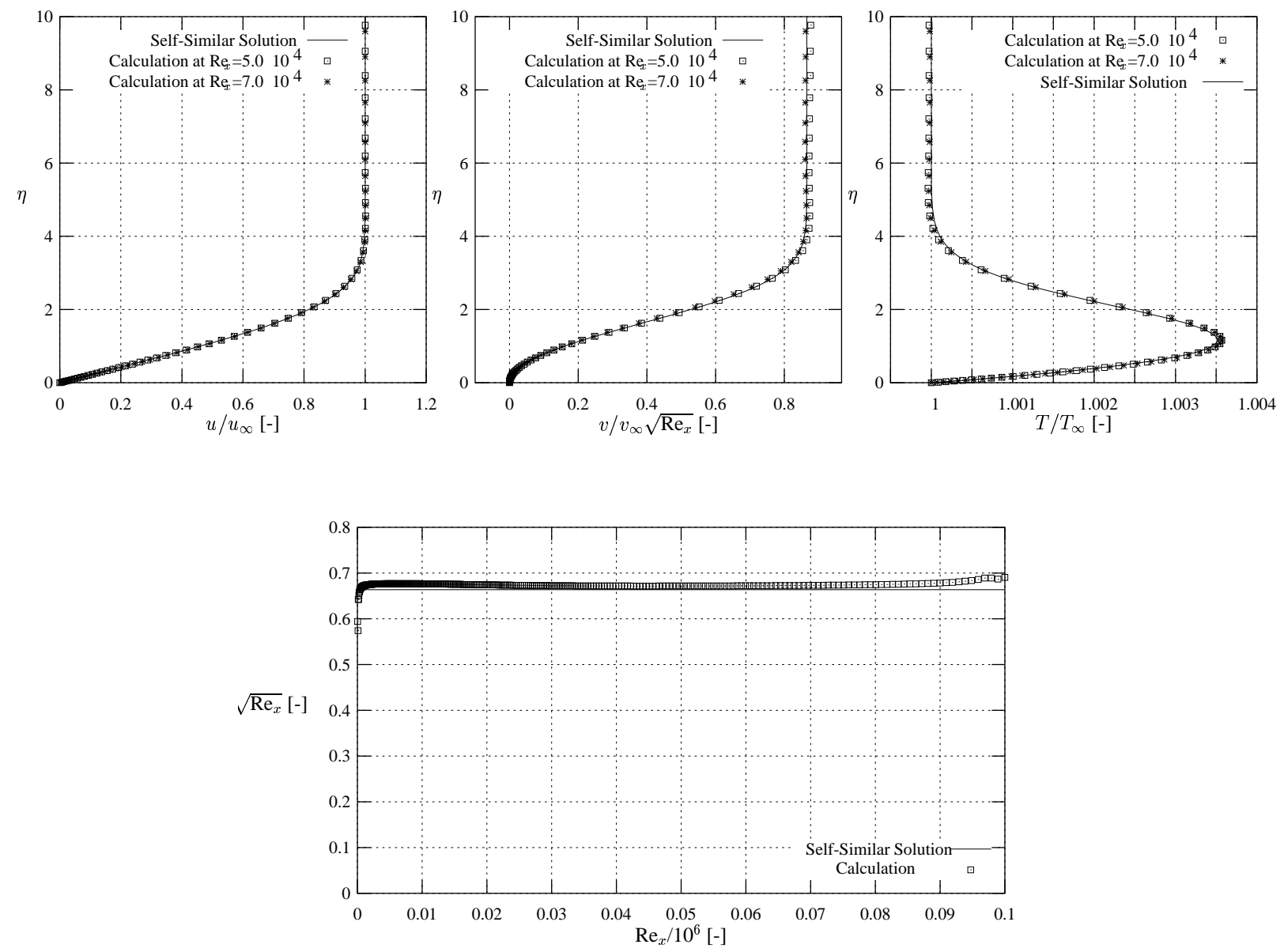

Figure 5.12: Comparison of numerical and analytical solutions of the self-similar boundary layer of a flat plate at zero pressure gradient at $M a_{\infty}=0.3$ and $R e_{L}=10.0^{5}$

top left: velocity $u$ in the main flow direction, top center: velocity $v$ in the wall normal direction, top right: temperature $T$, bottom: skin-friction coefficient $c_{f}$ vs. $R e_{x}$ 


\section{Turbulent Flow}

A detailed investigation of the plane incompressible turbulent COUETTE flow using dimensional analysis, estimating the order of molecular and turbulent momentum transfer and the subdivision of the flow into two layers led to the universal law of the wall $[31,106]$, which is independent of the choice of turbulence models. The friction velocity

$$
u_{\tau}=\sqrt{\frac{\tau_{w}}{\rho}}
$$

at a certain wall shear stress $\tau_{w}$ is the characteristic velocity for turbulent flows. By introducing the dimensionless values

$$
u^{+}=\frac{\tilde{u}}{u_{\tau}} \quad \text { and } \quad y^{+}=\frac{y u_{\tau}}{\nu}
$$

four relevant regions can be defined

$$
\begin{array}{rcc}
\text { viscous sublayer: } & 0 \leq y^{+}<5 & u^{+}=y^{+} \\
\text {transitional layer: } & 5 \leq y^{+} \lesssim 70 & \\
\text { log layer: } & 70 \lesssim y^{+}<1000 & u^{+}=\frac{1}{\kappa} \ln y^{+}+C \\
\text { defect layer: } & 1000 \leq y^{+} &
\end{array}
$$

with the constants $\kappa \approx 0.41$ and $C \approx 5.0$ determined in many experiments for hydraulically-smooth surfaces. For a detailed overview on the constants see [31]. Further relations $u^{+}\left(y^{+}\right)$for the different regions can be found in $[31,67,116]$. Based on the Morkovin hypothesis [66] for small MACH numbers $M a_{\infty}<5$ and according to the analysis of VAN DRIEST [131] these results are also valid for compressible flows with minor modifications to the values of $\kappa$ and $C$ in equation (5.15). Following [106, 131] the skin-friction coefficient $c_{f}$ for the turbulent boundary layer can be given implicitly for compressible flows

$$
\frac{0.242}{\sqrt{c_{f}}}\left(1+\frac{\gamma-1}{2} M a_{\infty}^{2}\right)^{-\frac{1}{2}}=\log \left(R e_{x} c_{f}\right)-\frac{1}{2} \log \left(1+\frac{\gamma-1}{2} M a_{\infty}^{2}\right)
$$

Results of the turbulent boundary layer at $M a_{\infty}=0.3$ and $R e_{L}=10.0^{6}$ are shown in figure 5.13 in comparison with the theoretical law of the wall and the theoretical $c_{f}$ distributions. Simulations with the new turbulence model are juxtaposed to the SPALART-Allmaras model on different grid resolutions. The grid resolution study shows that the law of the wall is well predicted even on very coarse grids for both turbulence models. The minimum grid spacing in the wall normal direction for the very coarse grid has the normalized size $\Delta y^{+} \approx 1$ and a maximum stretching factor normal to the wall of approximately 2.6. The good behavior of the turbulence models on relatively coarse grids is one of the key factors for the success of turbulence models such as the SPALART-Allmaras model. 


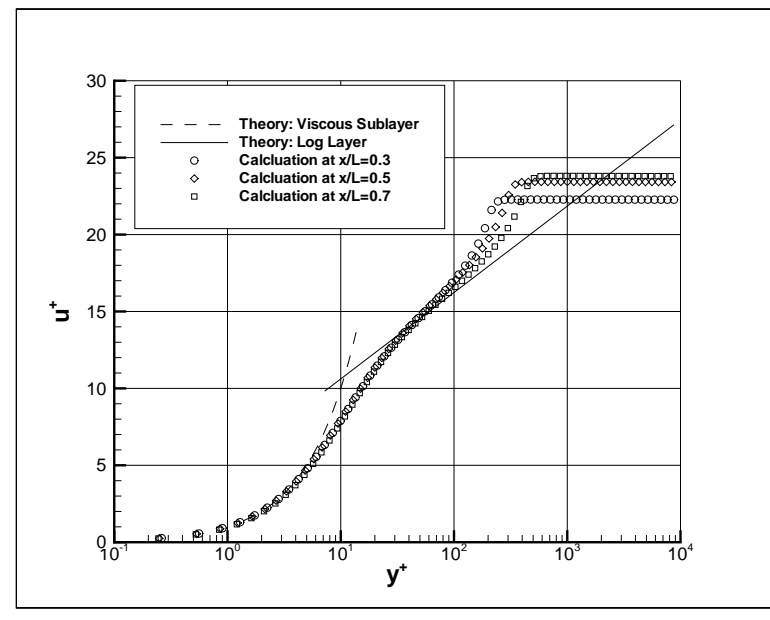

new model

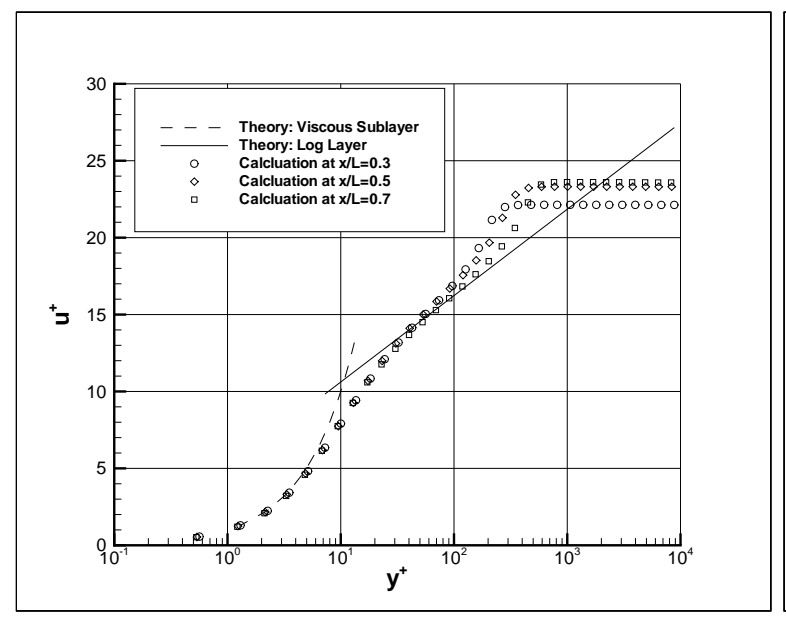

new model

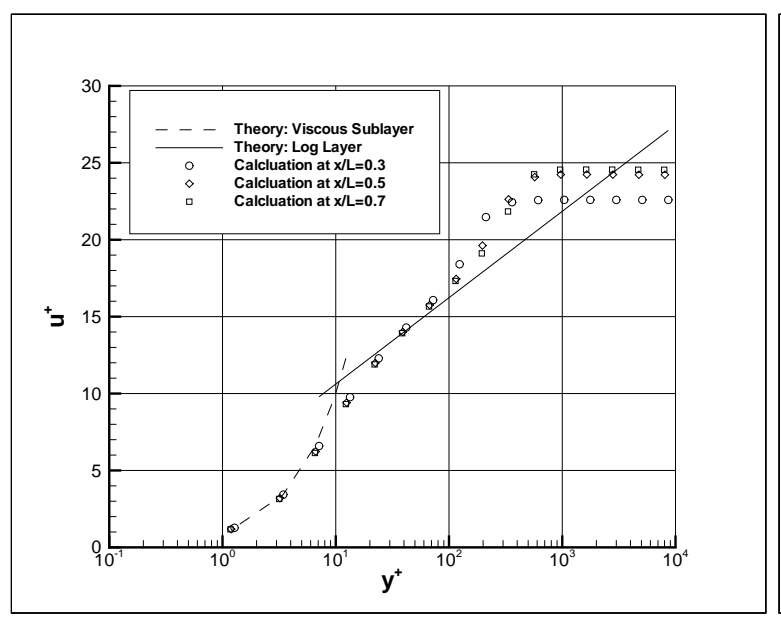

new model

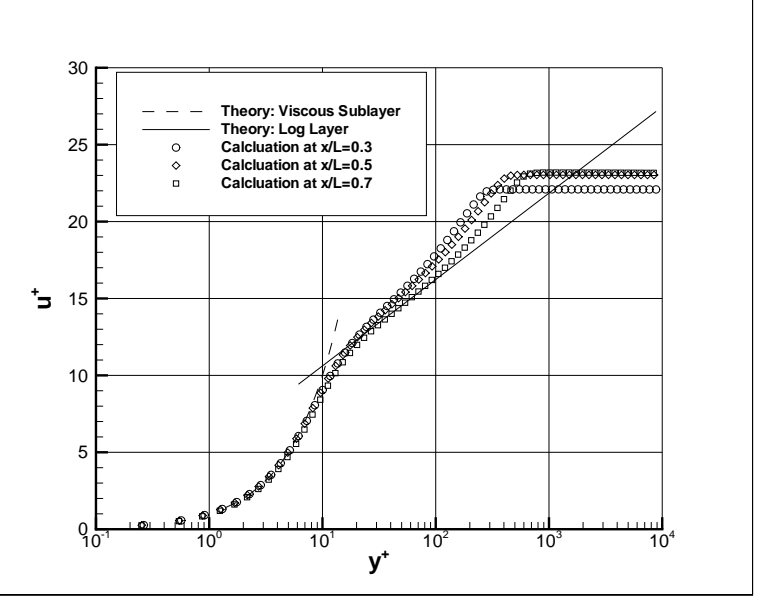

SPALART-ALLMARAS model

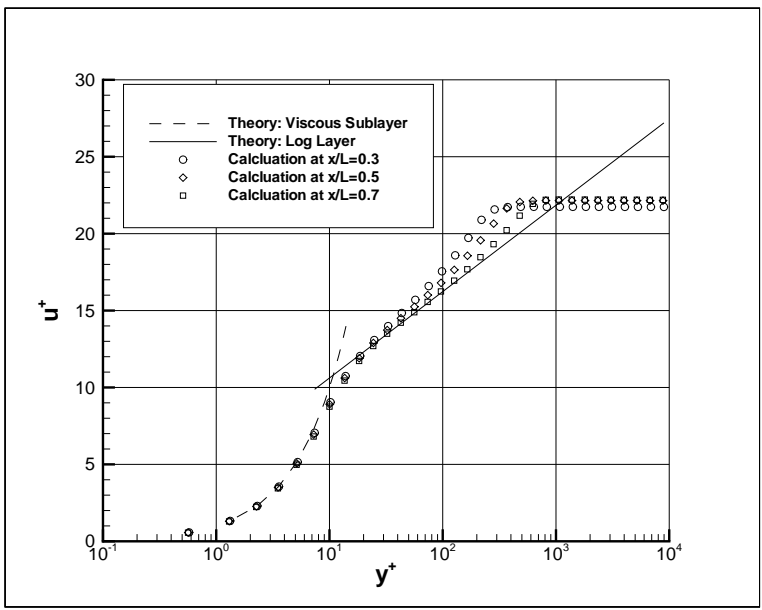

SPALART-AlLMARAS model

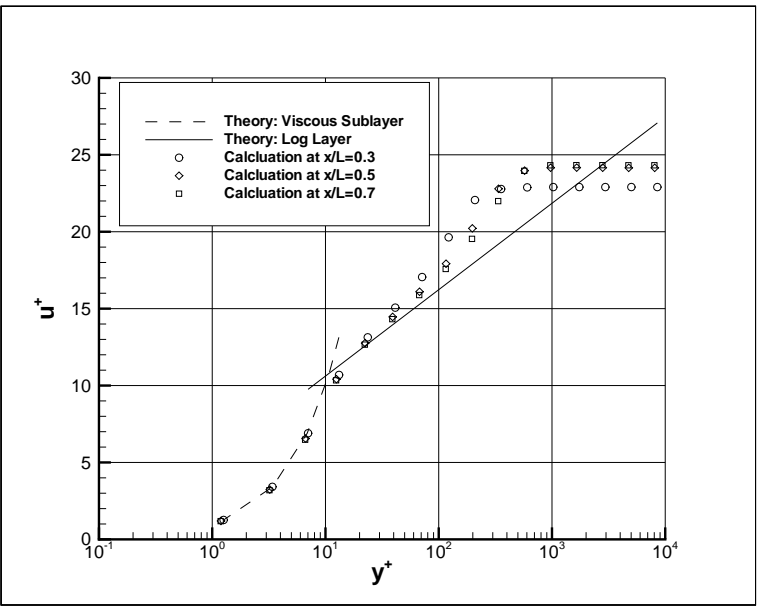

SPALART-ALLMARAS model

Figure 5.13: Comparison of numerical findings and the law of the wall of the turbulent boundary layer of a flat plate at $M a_{\infty}=0.3$ and $R e_{L}=10.0^{6}$ at different grid resolution

top: $(129 \times 65), \quad$ middle: $(65 \times 33), \quad$ bottom: $(33 \times 17)$ 


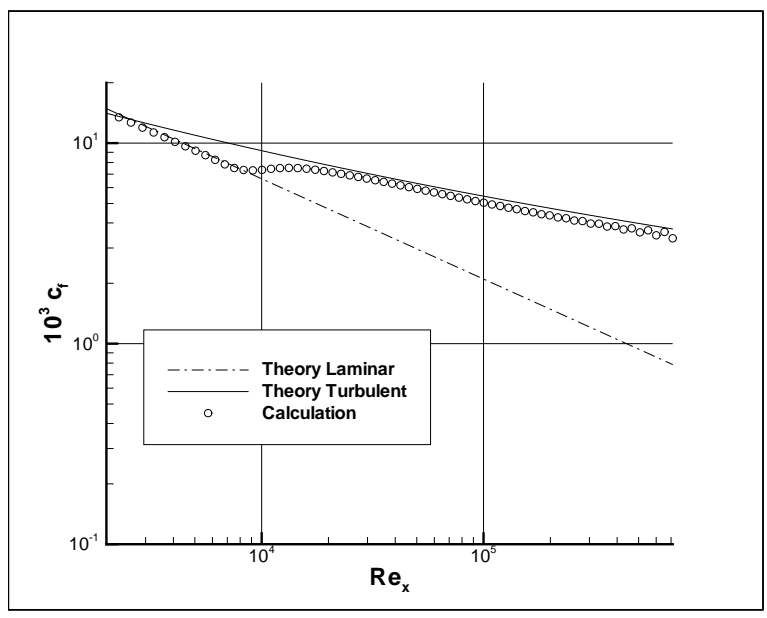

new model

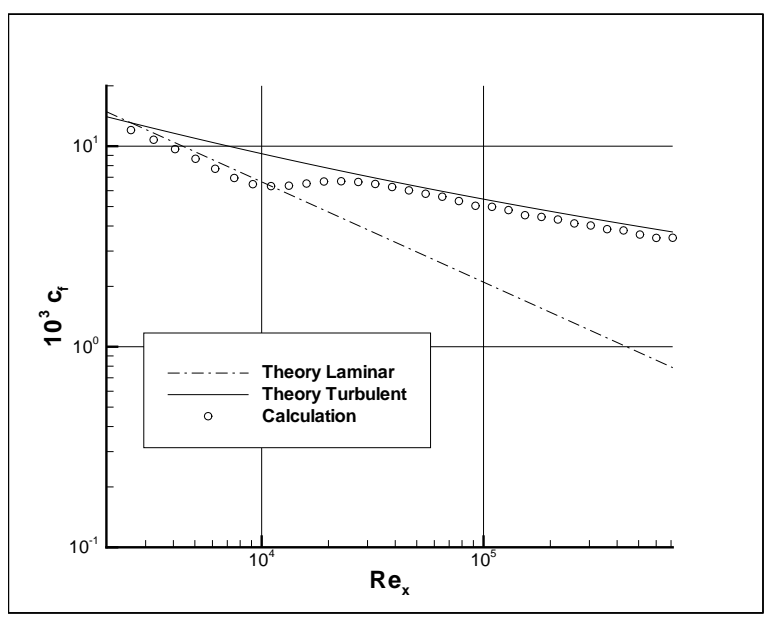

new model

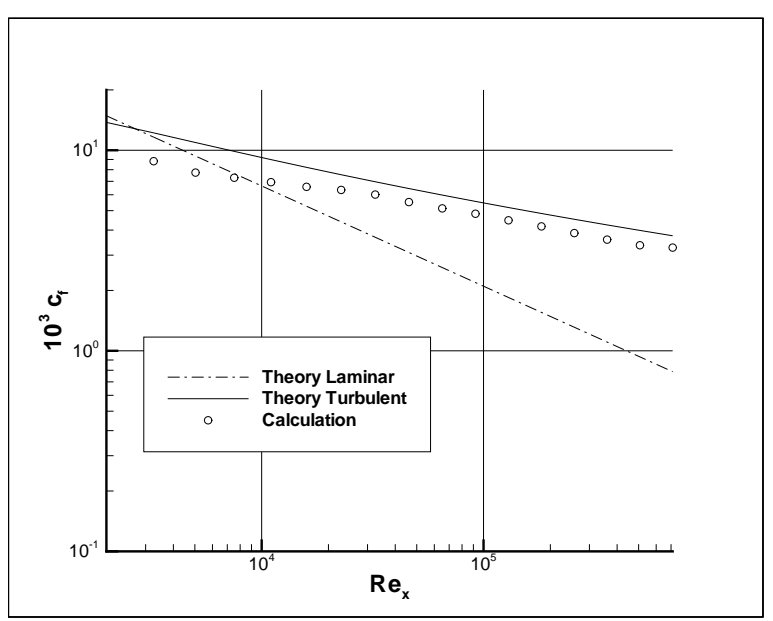

new model

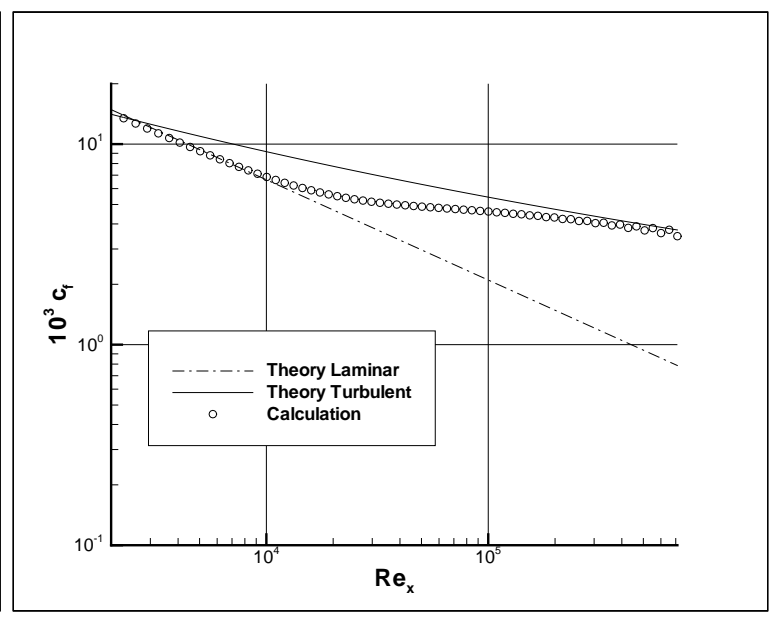

SPALART-AlLmaras model

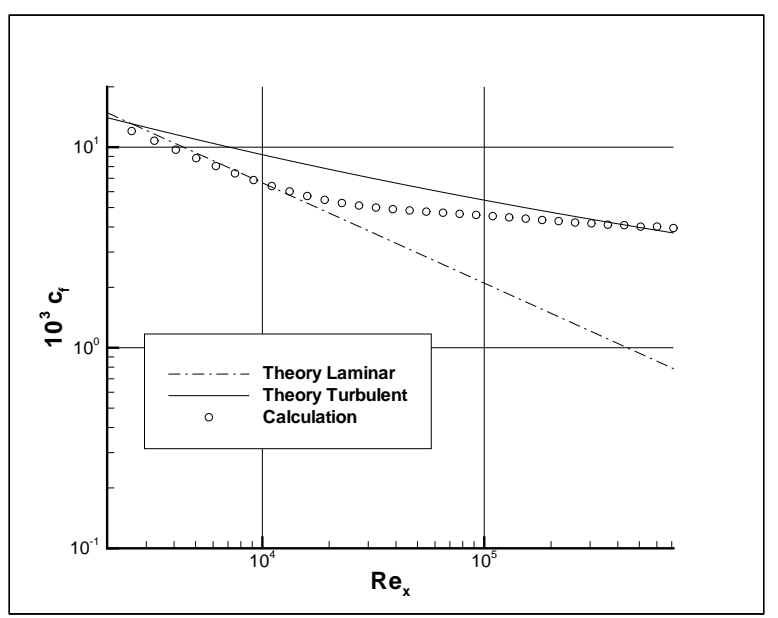

SPALART-AlLmaras model

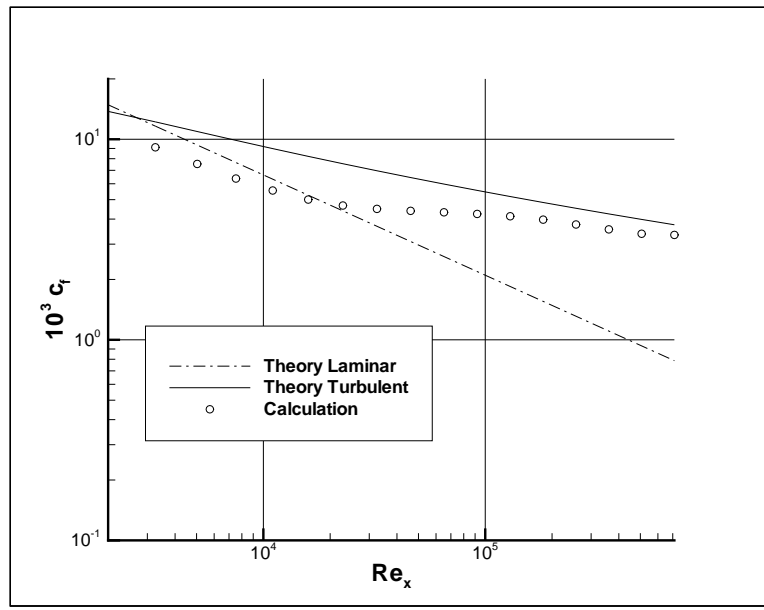

SPALART-ALLMARAS model

Figure 5.14: Comparison of numerical findings of the turbulent boundary layer of a flat plate at $M a_{\infty}=0.3$ and $R e_{L}=10.0^{6}$, grid resolution $(65 \times 33)$ and theoretical laminar (equation 5.11) and turbulent (equation 5.16) $c_{f}$ distributions at different grid resolution top: $(129 \times 65), \quad$ middle: $(65 \times 33), \quad$ bottom: $(33 \times 17)$ 
The skin-friction distributions $c_{f}$, which are depicted is logarithmic scales in figure 5.14 point out the transitional behavior of the turbulence model along the flat plate. Both models predict a transition from a laminar to a turbulent boundary layer. The SPALARTAllmaras model has a long transition period, whereas the new model possesses a much smaller region of transition. It occurs at a REYNOLDS number almost one order of magnitude below the natural transition of $R e_{\text {transition }}=3.5 \cdot 10^{5}$. Following the arguments of SPALART [110] the transition predicted by turbulence models lacking a detailed stability analysis are not generally valid and should not be trusted. It is, however, usually advantageous to have a quicker transition near the leading edge as in the case of the new turbulence model to allow a comparison with fully turbulent flows. It should also be noted that the turbulent skin-friction coefficient $c_{f}$ predicted by the new turbulence model is slightly smaller than the theoretical distribution. This is due to the chosen viscous damping function $f_{v 1}$. Nevertheless, the function $f_{v 1}$ is a good compromise since it does not depend on any wall distance $d$ and still gives acceptable distributions even on the very coarse grid compared to the SPALART-ALLMARAS model, which has a wall distance dependence, that becomes evident when the distributions of figure 5.14 are juxtaposed.

\section{Turbulent Flow with Pressure Gradient}

Typical boundary layers have pressure gradients. There exist a wide range of test cases to measure the predictive capabilities of turbulence models for such flows. The following figures 5.15-5.20 show the velocity and the skin-friction coefficient distributions predicted by the $k-\omega$, the SPALART-Allmaras and the new turbulence model compared to measurements. The test cases were defined at the AFSOR-IFP-STANFORD conference on the computation of Turbulent Boundary Layers [50]. Table 5.2 gives an overview on the calculated flows and the experiments belonging to them.

\begin{tabular}{|l|c|c|}
\hline Flow & description & Experiment \\
\hline \hline Flow1300 & favorable pressure gradient & {$[59]$} \\
\hline Flow6300 & favorable pressure gradient & taken from $[139]$ \\
\hline Flow1100 & weak adverse pressure gradient & {$[59]$} \\
\hline Flow2100 & weak adverse pressure gradient & {$[108]$} \\
\hline Flow0141 & increasingly adverse pressure gradient & {$[97]$} \\
\hline Flow1200 & strong adverse pressure gradient & {$[59]$} \\
\hline
\end{tabular}

Table 5.2: $\quad$ Flow spreading rates for turbulent free shear flows

The figures prove the capability of the proposed turbulence model to compete with the other models. The new model behaves either like the $k-\omega$ or the SpALART-Allmaras model. Although the skin-friction coefficient distributions is somewhat overpredicted for the adverse pressure gradient flows (figures 5.17,5.19,5.20) it is fair to conclude that it yields in all cases satisfactory results. 

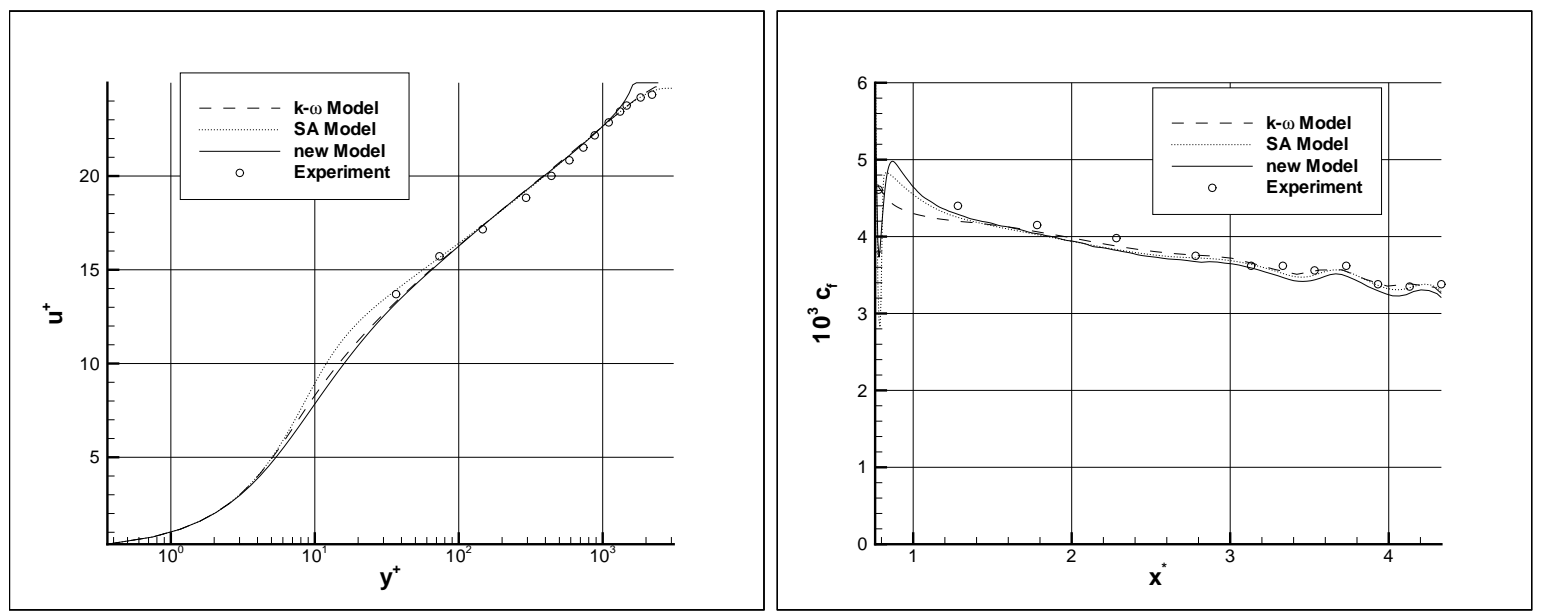

Figure 5.15: Computed and measured velocity (left) and skin-friction (right) for boundary layer flow at a favorable pressure gradient (Flow $1300[59,139])$
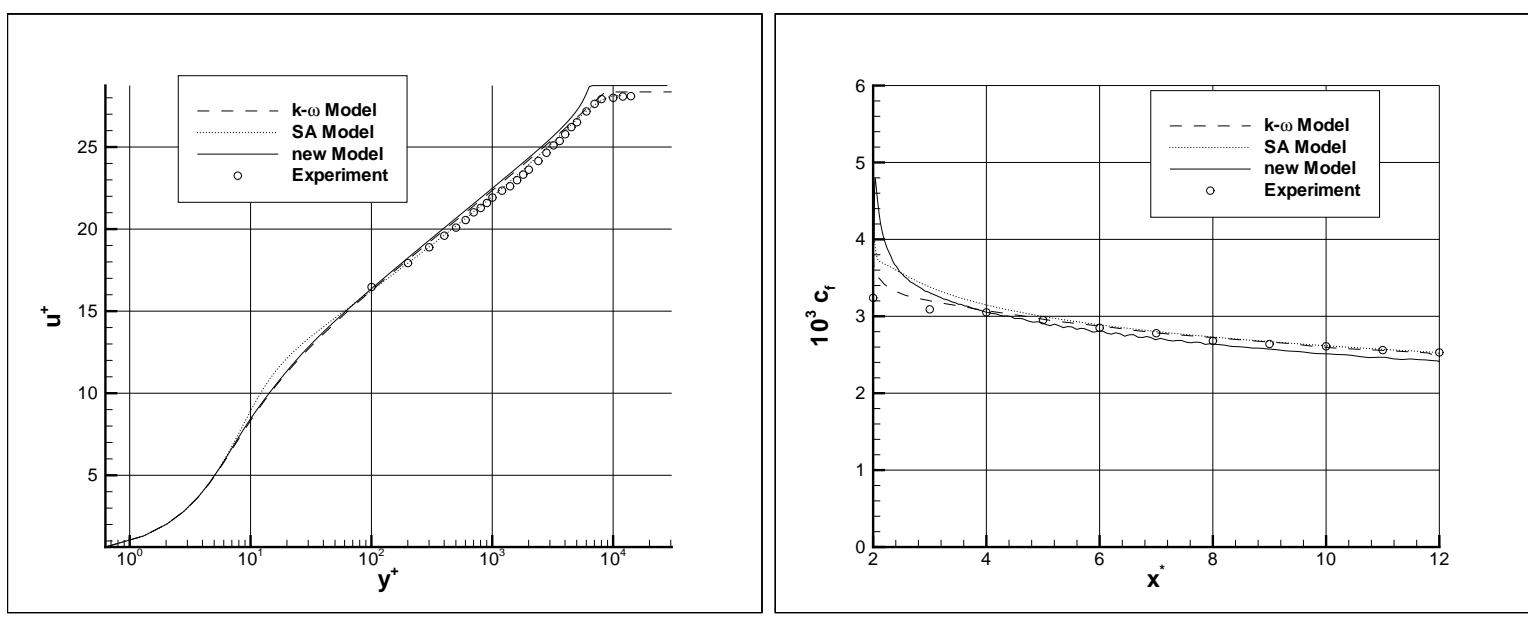

Figure 5.16: Computed and measured velocity (left) and skin-friction (right) for boundary layer flow at a favorable pressure gradient (Flow 6300 [139])
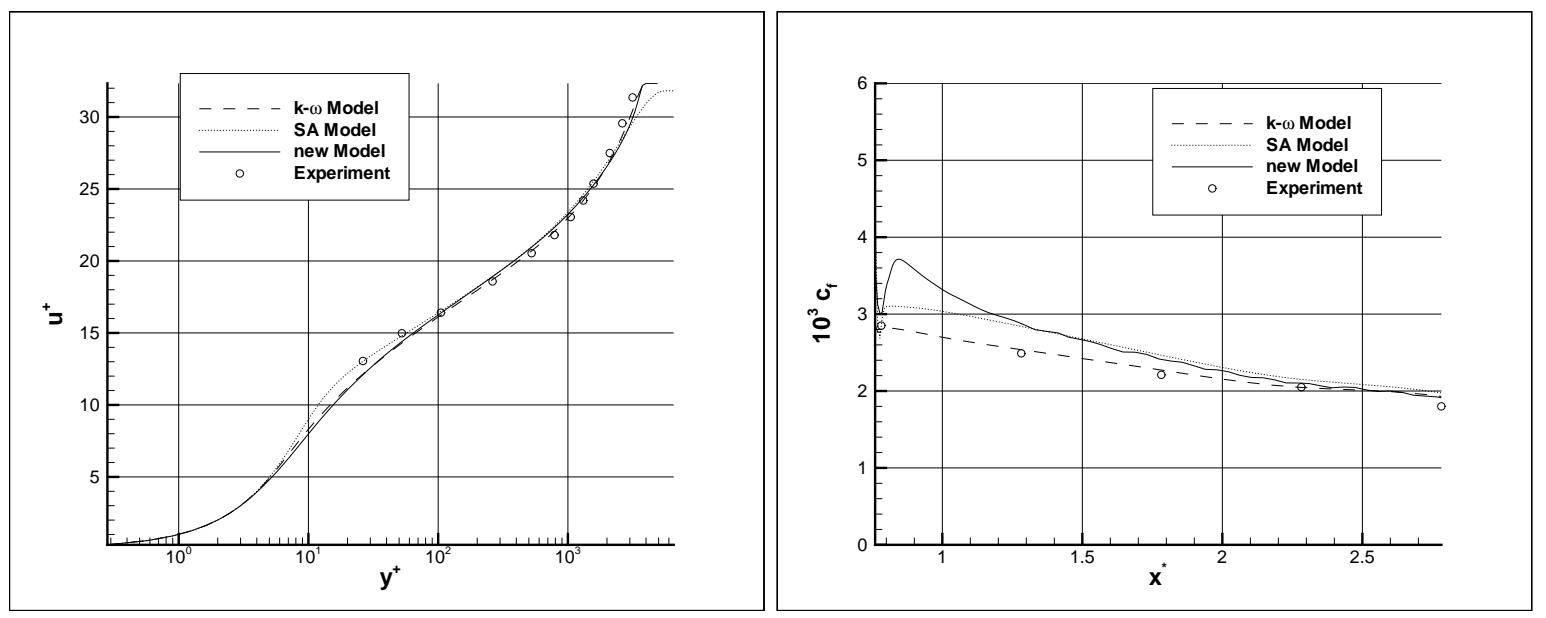

Figure 5.17: Computed and measured velocity (left) and skin-friction (right) for boundary layer flow at a weak adverse pressure gradient (Flow $1100[59,139]$ ) 

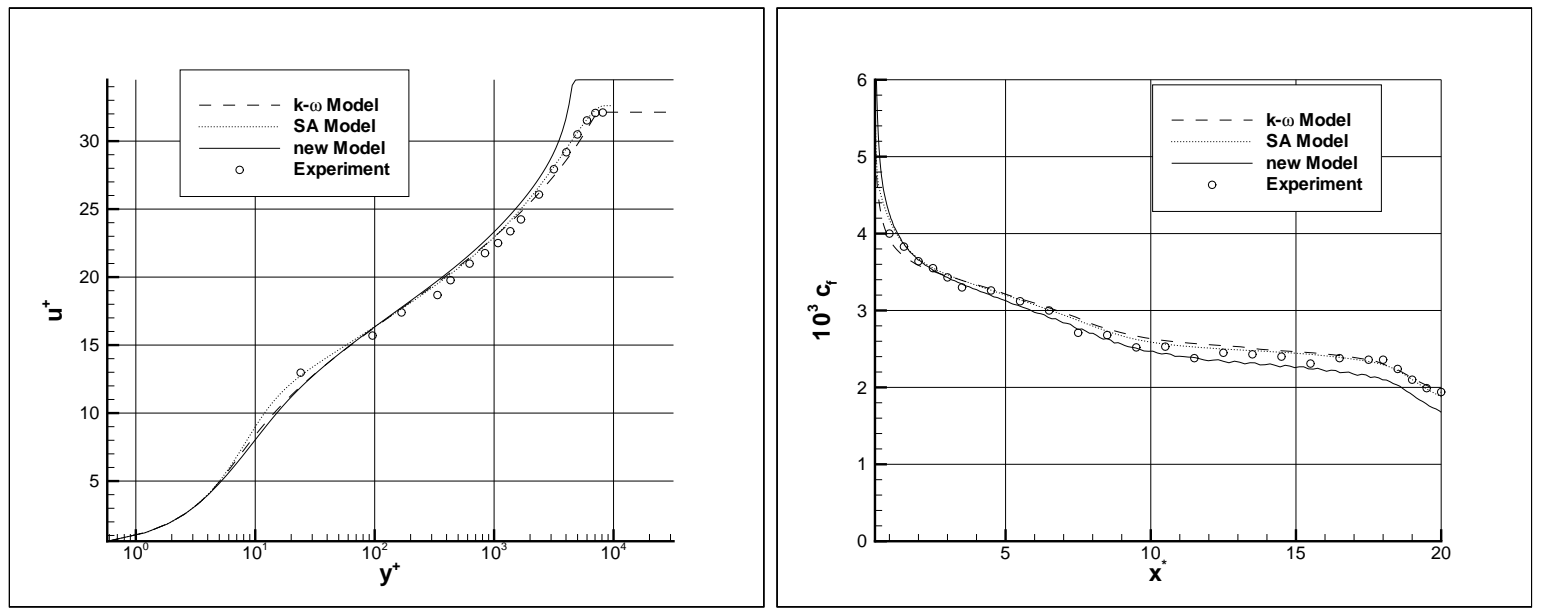

Figure 5.18: Computed and measured velocity (left) and skin-friction (right) for boundary layer flow at a weak adverse pressure gradient (Flow 2100 [108, 139])
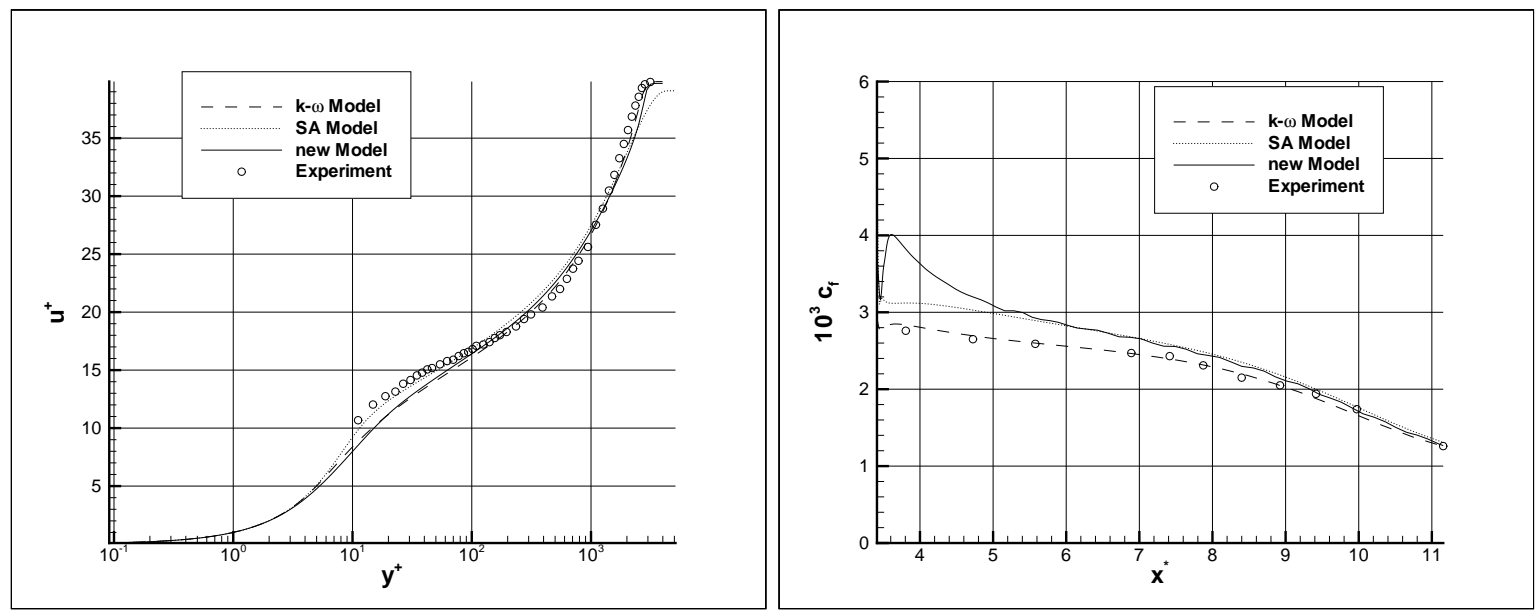

Figure 5.19: Computed and measured velocity (left) and skin-friction (right) for boundary layer flow at an increasingly adverse pressure gradient (Flow $0141[97,139])$
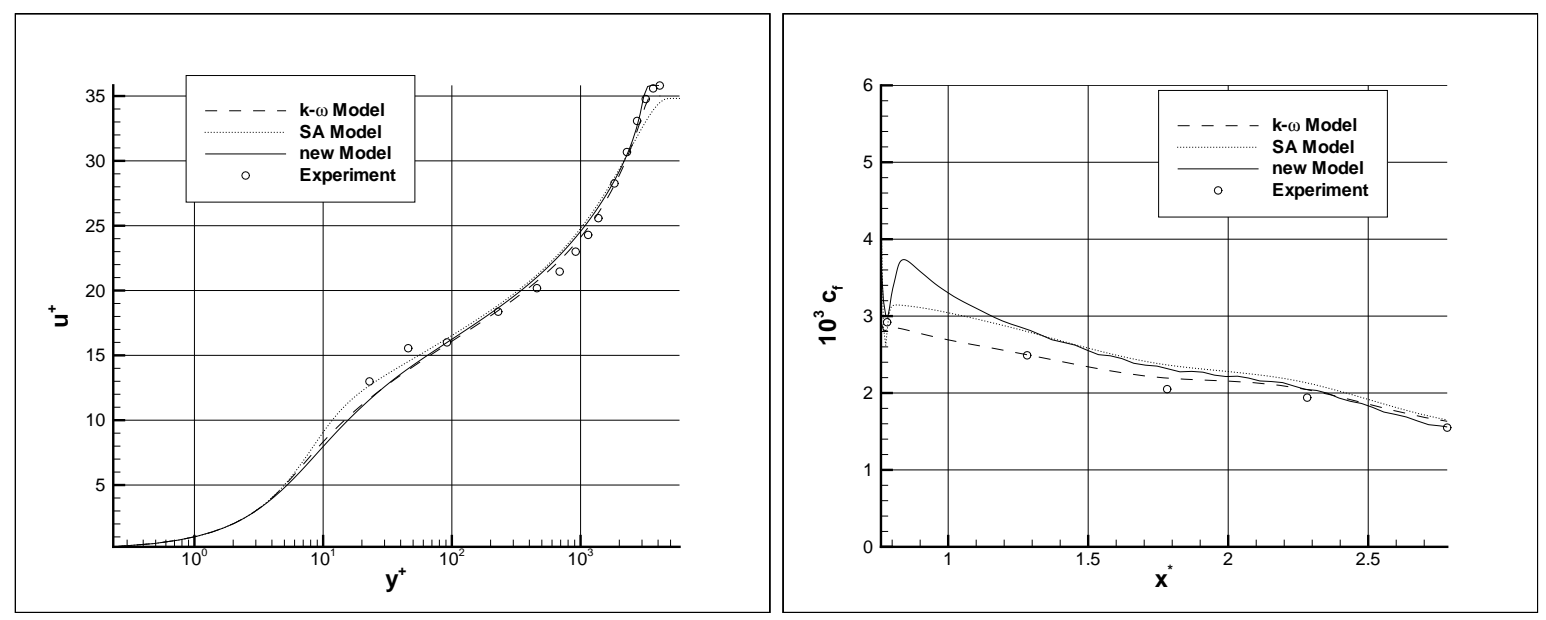

Figure 5.20: Computed and measured velocity (left) and skin-friction (right) for boundary layer flow at a strong adverse pressure gradient (Flow 1200 [59, 139]) 


\subsubsection{Aerodynamic Flows}

The analysis of the prediction of a flow over a clean airfoil or a high lift configuration is a must to discuss the quality of the turbulence model and the numerical algorithm in general. Problems associated with grid generation also play a major role but a thorough investigation is beyond the scope of this study.

\section{RAE 2822 Airfoil}

The transonic turbulent flow over the $R A E$ 2822 airfoil, which was experimentally investigated in [12], represents a standard aerodynamic test case for turbulence models. The findings of the simulation of the flow at $M a_{\infty}=0.73, R e_{c}=6.5 \cdot 10^{6}$ and an angle of attack $\alpha=2.79^{\circ}$ using the SPALART-AllmarAs and the new turbulence model are depicted in figure 5.21. The distribution of the pressure coefficient distribution $c_{p}$ of the new turbulence model shows better agreement with the experiments than the SpalarT-Allmaras model especially near the shock region on the upper surface and at the trailing edge region on the lower surface. A similar result as far as the deviation of the data of the SPALART-AlLmaras model and the measurements is concerned is shown in the original paper of Spalart and Allmaras [110]. For the $c_{f}$ distribution the new model yields a slightly better agreement upstream of the shock region, whereas the SPALART-ALlmaRAS model shows better predictions downstream of the shock. It can be conjectured that the stronger shock predicted by the SPALARTAllmaras leads to a smaller local MACH number after the shock. Consequently, the local velocity gradients normal to the wall and hence the skin-friction is smaller than that determined by the new turbulence model. It can be seen by the almost identical local MACH number contours near the airfoil for both turbulence models in figure 5.22 that this result is very local and has hardly any impact on the overall flow field.
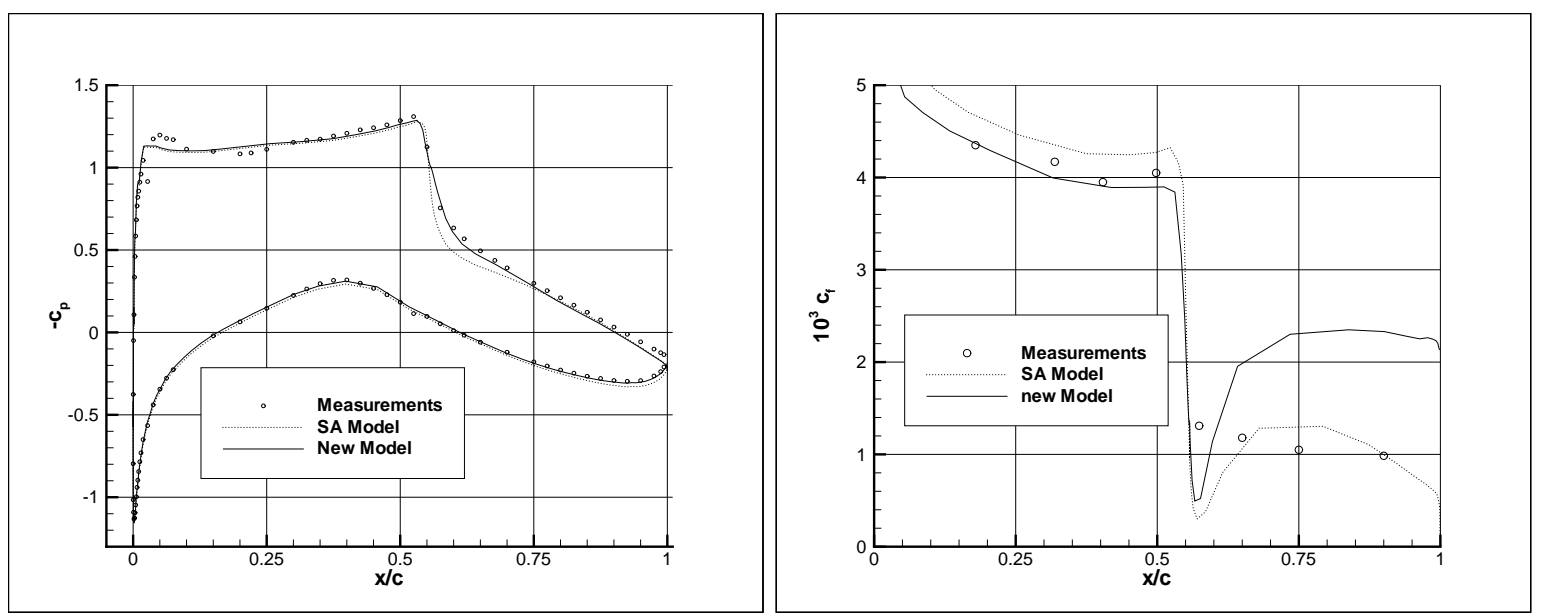

Figure 5.21: Computed and measured pressure $c_{p}$ distribution (left) and skin-friction coefficient $c_{f}$ distribution (right) of the $R A E 2822$ airfoil at $M a_{\infty}=0.73, R e_{c}=6.5 \cdot 10^{6}$ and $\alpha=2.79^{\circ}$ 


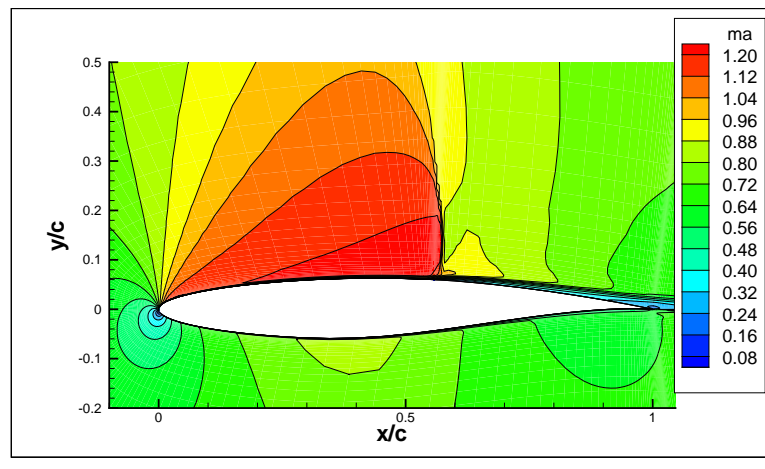

new model

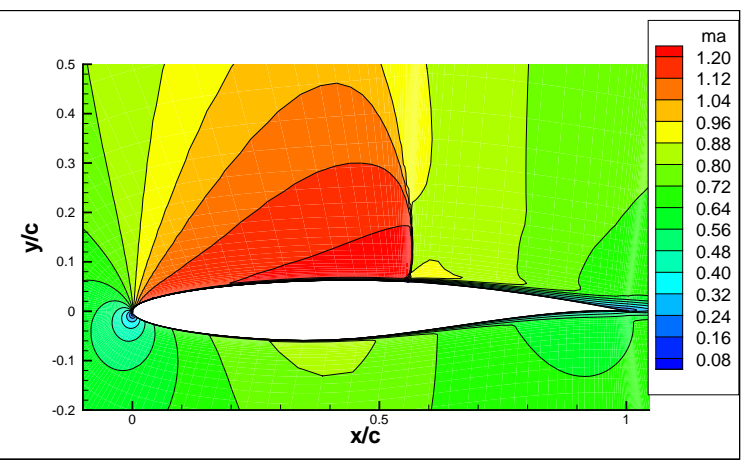

SPALART-AlLmaras model

Figure 5.22: Computed MACH number contours of the $R A E$ 2822 airfoil at $M a_{\infty}=0.73$, $R e_{c}=6.5 \cdot 10^{6}$ and $\alpha=2.79^{\circ}$

\section{High Lift Configuration}

The two-dimensional flow of the multi-element airfoil still constitutes a challenging task for the computational fluid dynamics community since details like grid quality and turbulence modeling play a significant role in the correct simulation of the flow. The airfoil geometry is the $B A C 3-11$ in high lift configuration $(L 1 / T 2)$ as described in [65]. The geometry and the multiblock grid used for the numerical simulation is shown in figures 4.3 and 5.23. The clean configuration of this airfoil constitutes the basic geometry for the study in this work and was investigated in former analyses [25, 26] within the collaborative research program $S F B 401$.

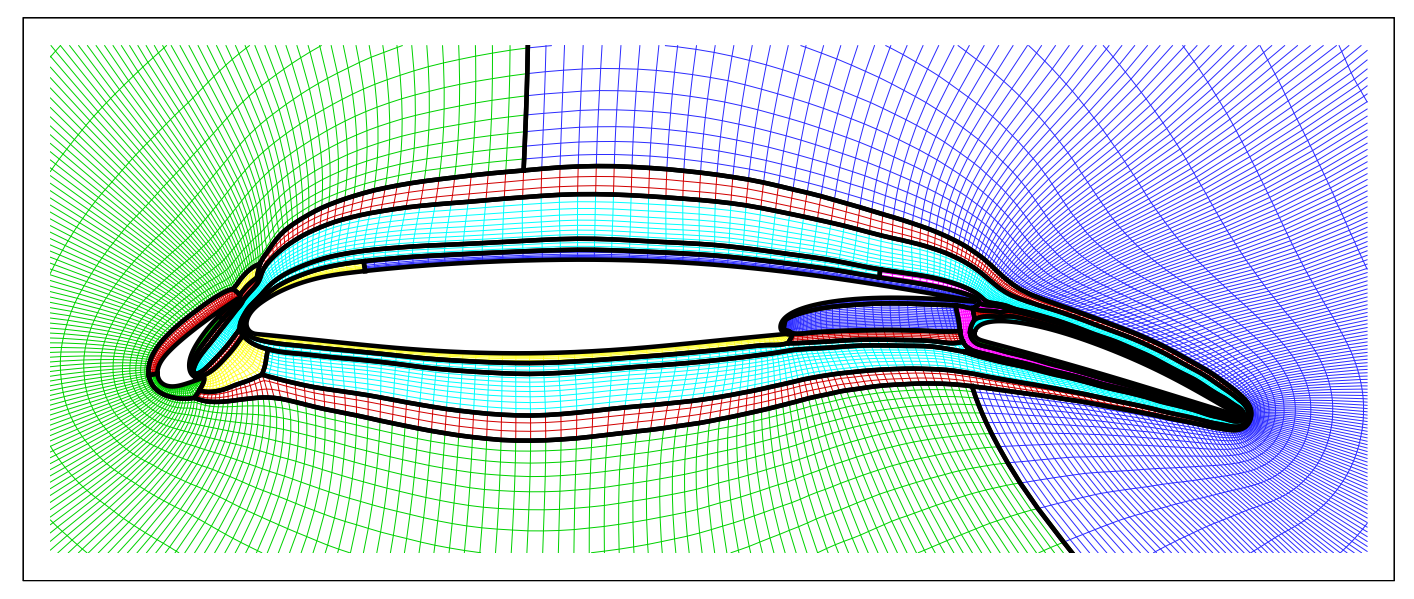

Figure 5.23: Multiblock grid of the three element airfoil, 19 blocks, 42000 nodes

Figure 5.24 depicts the pressure coefficient distribution on the surface compared to experimental data from Moir [65] at $M a_{\infty}=0.198, R e_{c}=3.52 \cdot 10^{6}$ and $\alpha=4^{\circ}$. Except in the recirculation area on the slat lower surface a good agreement is achieved with the new model showing a somewhat closer correspondence with the experimental 

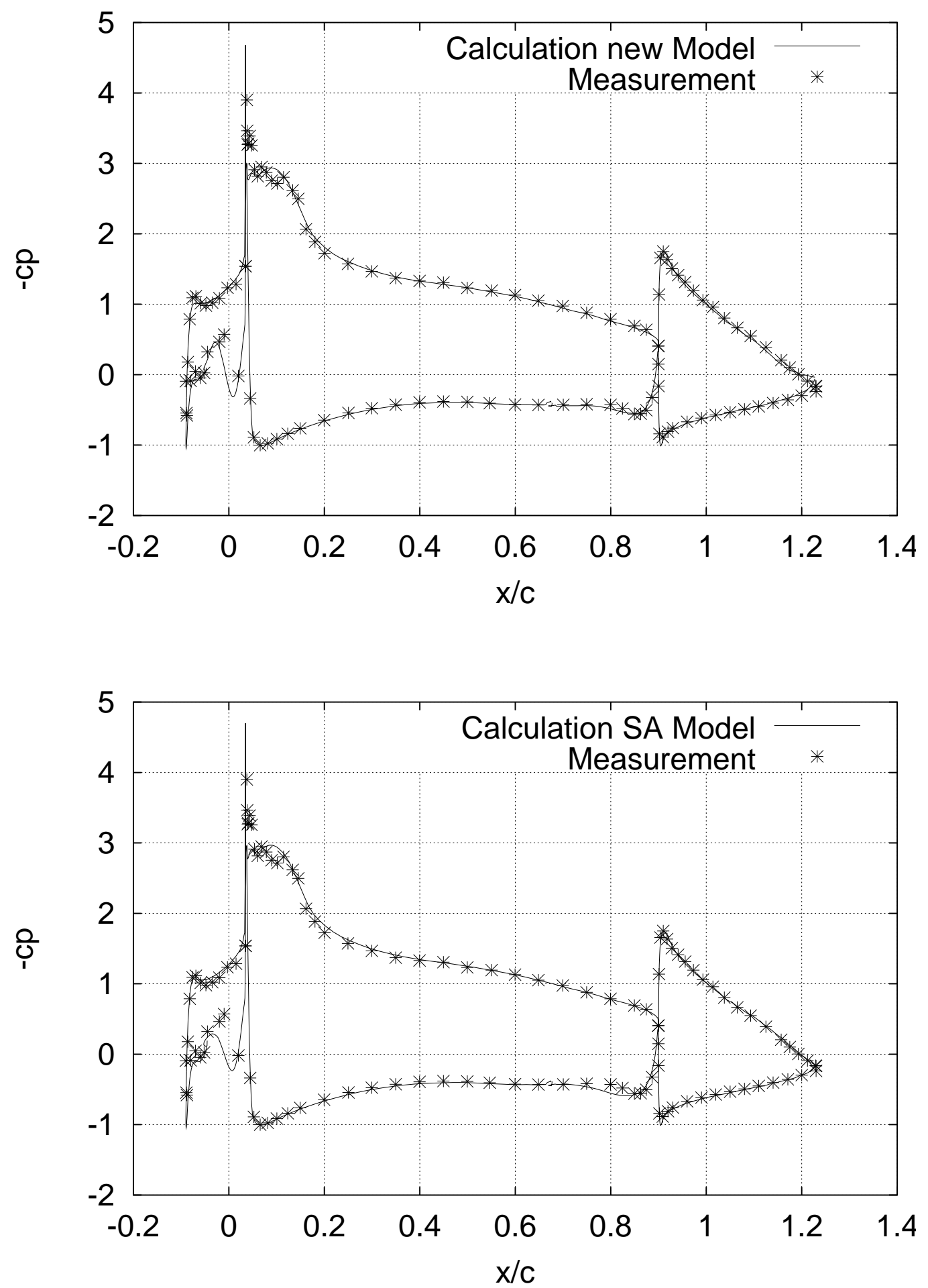

Figure 5.24: Computed and measured pressure coefficient $c_{p}$ distributions over the $B A C$ 3-11 airfoil in high lift configuration at $M a_{\infty}=0.198, R e_{c}=3.52 \cdot 10^{6}$ and $\alpha=4^{\circ}$ determined by the new model (top) and the $S A$ model (bottom) 
data in this region. A separated flow occurs there that is unsteady and therefore not correctly captured by the simulation that uses local time stepping. The MACH number distributions in figure 5.25 show the well resolved wake of the slat and the main wing although no special grid refinement study was preformed. Furthermore, the distributions emphasize the almost identical simulation results of both turbulence models for the overall flow field.

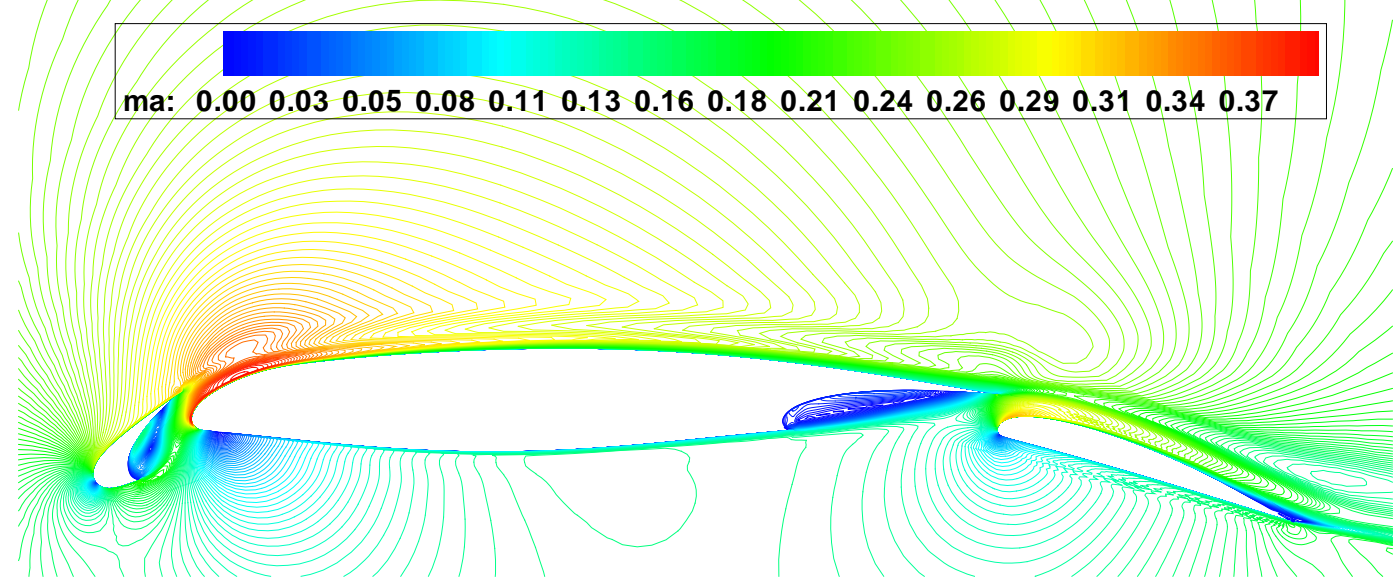

new model

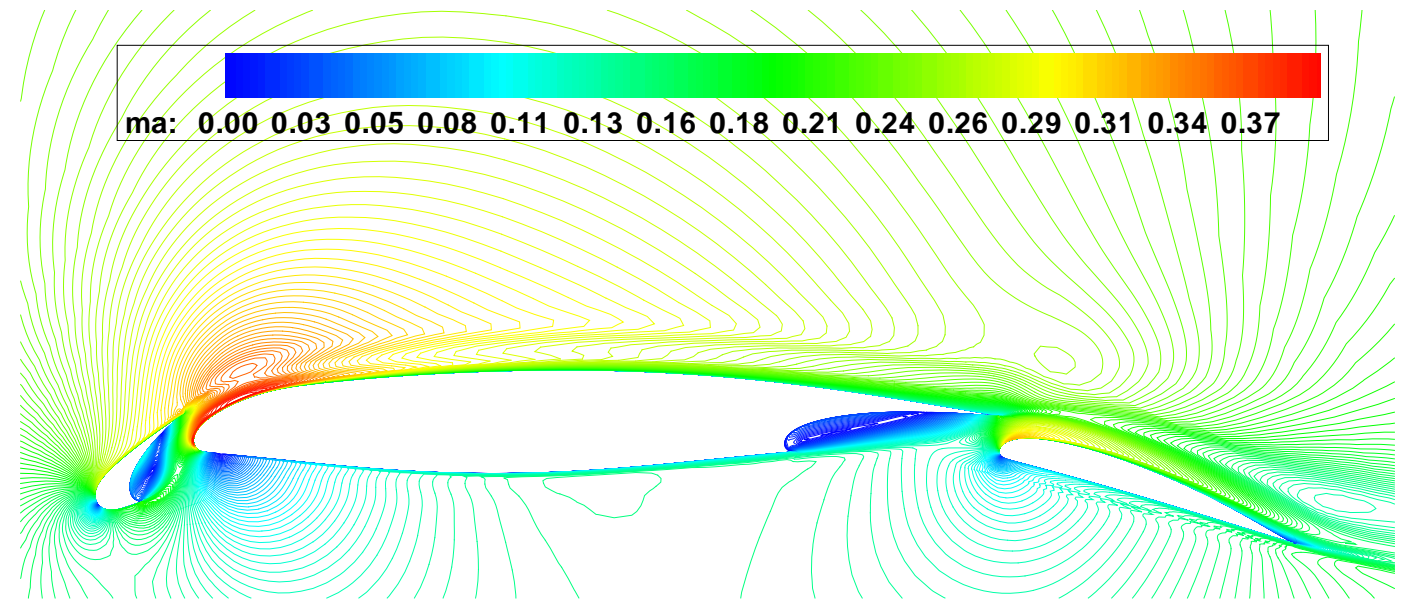

SPALART-AlLmaras model

Figure 5.25: Computed $\mathrm{MACH}$ number contours for the $B A C$ 3-11 airfoil in high lift configuration at $M a_{\infty}=0.198, \operatorname{Re}_{c}=3.52 \cdot 10^{6}$ and $\alpha=4^{\circ}$ 


\subsubsection{Wake of Airbus A300}

The wake of a complete A300 model was experimentally investigated by JACQUIN et al. in [46]. The experimental program included smoke visualization, Laser Doppler Velocimentry and hot wire measurements in the wake of the model in clean and in high lift configurations. The measurement points were located on a cartesian grid in 5 planes perpendicular to the freestream direction located at $x / b=0.5,1.0,3.0,5.0$ and 9.0 downstream of the trailing edge where $b$ is the wingspan. Special care was taken to locally refine the measurement grid to enhance the resolution of the vortex dominated regions.

The wake flow of the high lift configuration is also studied numerically for further validation of the numerical method in vortical flows. Compared with the clean geometry the high lift configuration represents due to the existence of several merging vortices the more challenging case. Two structured cartesian grids with 2.5 million grid points ranging from $x / b=0.5$ to $x / b=4.5$ and a 7.3 million points ranging from $x / b=0.5$ to $x / b=2.5$ were used for this investigation.

In this study measurements of the realistic wake are the basis of the calculation. Only the wake flow is investigated numerically. The measured velocity distribution in the plane $x / b=0.5$ is imposed explicitly at the inflow boundary. Since the numerical grid of the wake calculation usually possesses a finer resolution than the measurements, a second-order interpolation was used to compute additional values within the experimental domain and a third-order extrapolation for the points outside the experimental domain. It was found in cases with strong gradients of vorticity and velocity that even higher-order up to 4th order interpolation schemes were necessary to achieve the same level of vorticity on the fine grid. It has to be noted that the inflow distribution is very susceptible to the interpolation, i.e. different techniques such as higher order interpolation or weighted inverse distance schemes lead to strong differences in the interpolated data from the original data. The experimental resolution of the vortex structure is based on 5 points per diameter. An interpolation of this vortex on 20 discrete points per diameter using a linear interpolation led to half of the experimental vorticity magnitude and a third-order interpolation led to approx. 1.5 the experimental vorticity. Note, however, that a higher vorticity may not be wrong, since the measurements could have missed the peak velocities and as such result in a lower vorticity. It is therefore necessary that the interpolated data ensure the physical consistency with the measured data.

Furthermore, the flow was assumed incompressible with a constant density $\rho_{\infty}$ at the inflow, since the freestream Mach number $M a_{\infty}$ is equal to 0.13 . Thus, the pressure can be calculated by solving the Poisson equation for an incompressible two-dimensional flow

$$
\nabla^{2} p=-\rho\left[\left(\frac{\partial u}{\partial x}\right)^{2}+\left(\frac{\partial v}{\partial y}\right)^{2}+2 \frac{\partial u}{\partial y} \frac{\partial v}{\partial x}\right] .
$$

At the boundaries of the integration domain the pressure $p_{\infty}$ was prescribed except for the symmetry plane where a Neumann boundary condition was imposed.

The measurements included the correlations and the RMS values of the fluctuations 
of the velocity components in the planes. To calculate a suitable initial condition for the turbulent viscosity $\nu_{t}$ several assumptions had to be made. The eddy viscosity is determined by

$$
\nu_{t}=C_{\mu} L \sqrt{k} \quad C_{\mu}=0.09
$$

where $L$ denotes the length scale and $k$ is the turbulent kinetic energy. Assuming the length scale $L$ to be of the order of the boundary layer thickness $\delta$ of the generating wing $L \approx \kappa \delta$ with $\kappa=0.41$ and approximating the boundary layer thickness [106] for the Reynolds number (based on the root chord length) $R e_{c}=200000$ the quantity $\nu_{t}$ can be estimated by

$$
\frac{\nu_{t}}{\nu} \approx \mathcal{O}(100) \cdot \sqrt{\frac{u^{\prime 2}+v^{\prime 2}+w^{\prime 2}}{u_{\infty}^{2}}} .
$$

The approximation resulted in values of $\nu_{t} / \nu \approx 15.0$ within the vortex cores, which is probably caused by the meandering of the vortices [11] and not by a real physical turbulent fluctuation. Nevertheless, these values were used as an initial condition since during the computation they are modified anyway to fulfill the conservation equation. The determination of the initial condition is valid only for one plane located at the inflow of the integration domain. To achieve a distribution on the whole domain tests were performed using a freestream condition initialization for the rest of the flow field. However, faster convergence was achieved by simply copying the inflow plane distribution throughout the entire computational domain.

The numerical solutions show qualitatively good agreement with measurements in the plane $x / b=1.0$ (figures 5.26-5.28). The comparison in figures 5.27 and 5.28 clearly demonstrates that the fine grid resolution is indeed needed to retain the high vorticity level. In figure 5.29 the experimental and numerical findings are juxtaposed in the plane $x / b=3.0$ and show that downstream merging of the flap and wingtip vortex has already taken place. The merging position lies just downstream of $x / b=2.5$ the plane which is visualized in figure 5.30 for the coarse and the fine grid. The differences in the shape of the merging process are caused by the discrepancies in the vorticity levels and the intensified numerical dissipation in the case of the coarse grid.

A quantitative analysis of the simulation is presented in figures 5.31 and 5.32. The profiles of the velocity component $u$ in the main flow direction and the tangential velocity $v$ as well as the vorticity through the wingtip and flap side edge vortices at $x / b=1.0$ confirm that the higher resolution is required to achieve the desired accuracy. The position of the vortices and their dynamics in the simulation slightly deviate from the experimental data. This may be due to the experimentally unsatisfied assumption of an exact symmetry of the flow and the missing influence of the wind tunnel walls in the numerical analysis. 

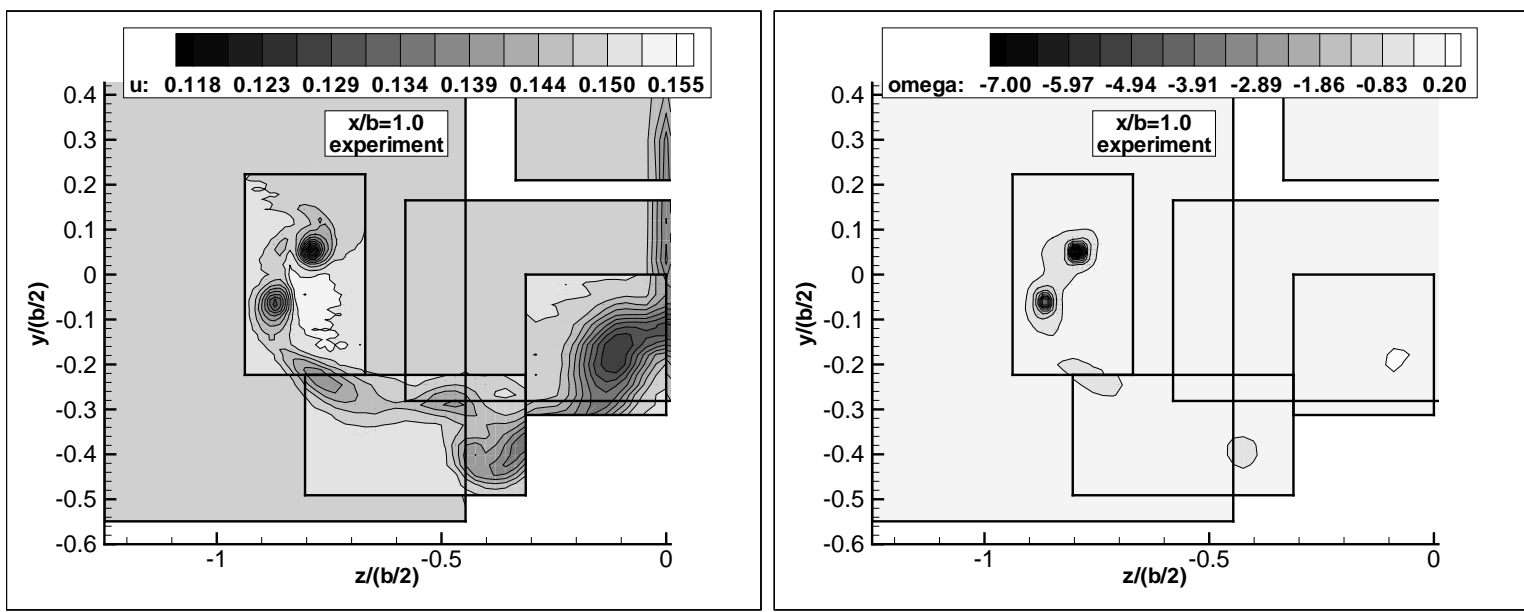

Figure 5.26: Measurements of the streamwise velocity component (left) and vorticity (right) of an A300 wake at $x / b=1.0$ for $M a_{\infty}=0.14$ and $R e_{c}=200000$
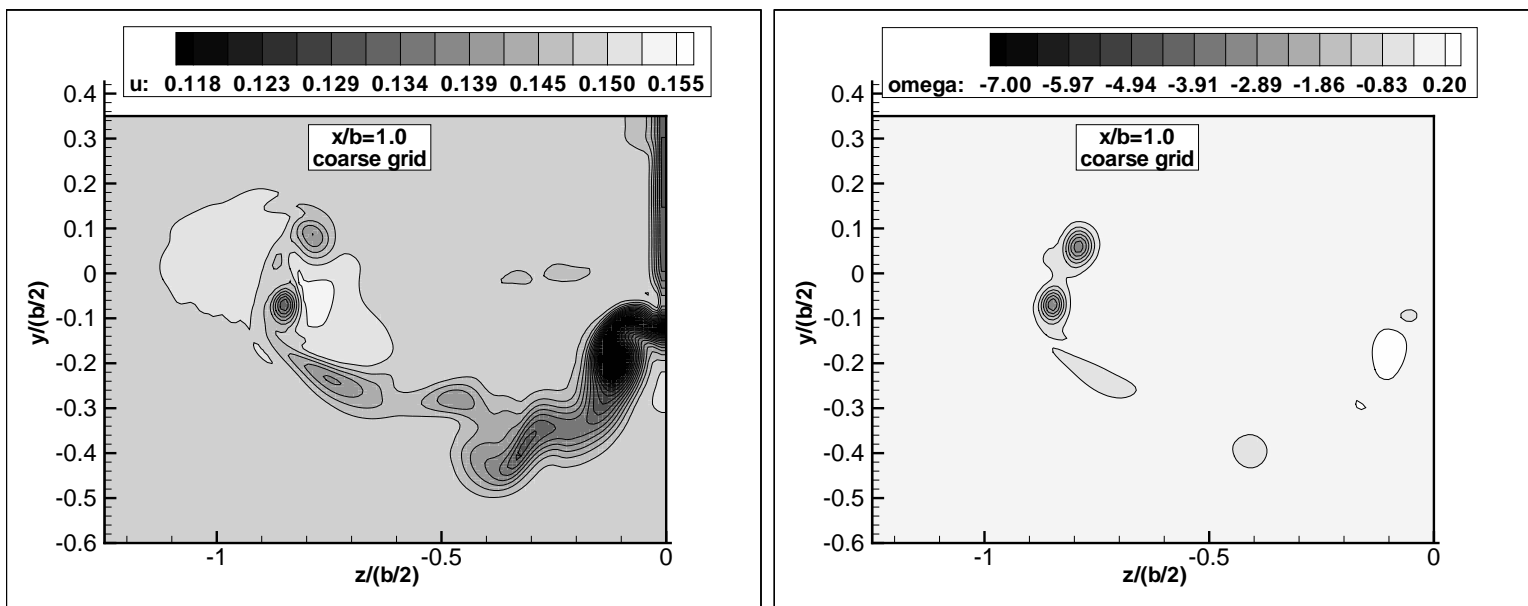

Figure 5.27: Numerical simulation on the coarse grid of the streamwise velocity component (left) and vorticity (right) of an A300 wake at $x / b=1.0$ for $M a_{\infty}=0.14$ and $R e_{c}=200000$
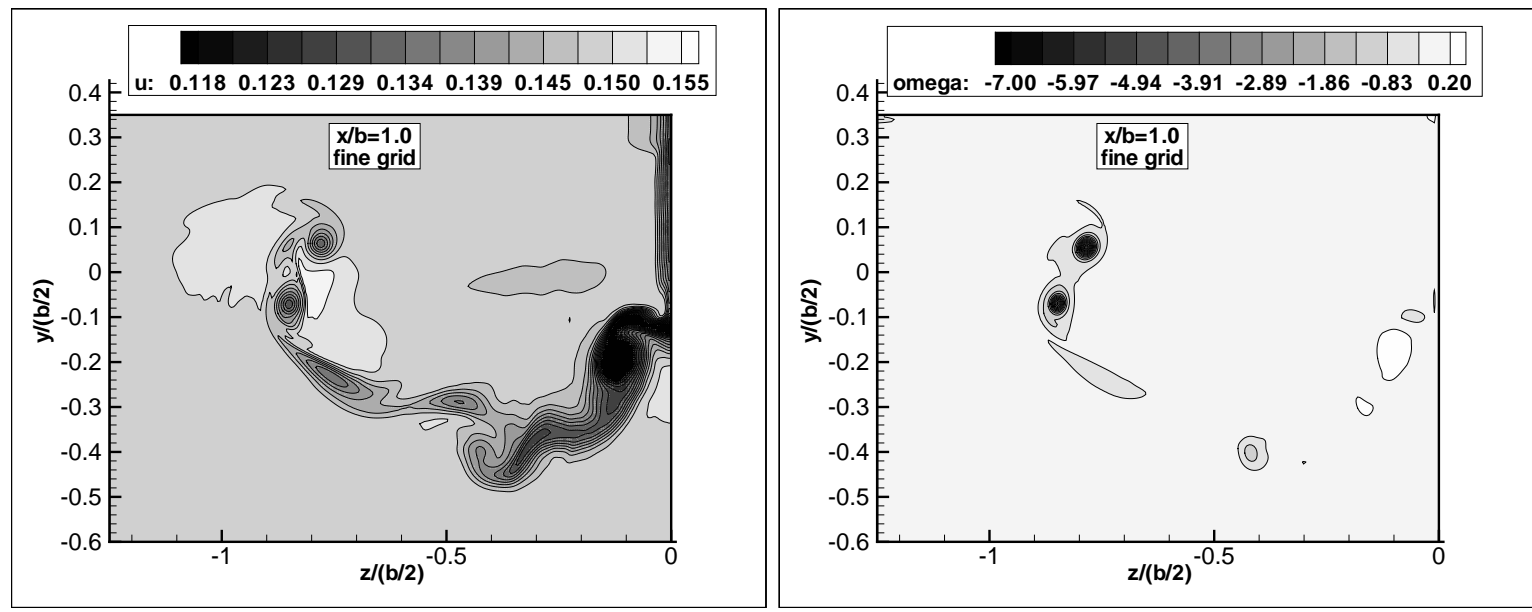

Figure 5.28: Numerical simulation on the fine grid of the streamwise velocity component (left) and vorticity (right) of an A300 wake at $x / b=1.0$ for $M a_{\infty}=0.14$ and $R e_{c}=200000$ 

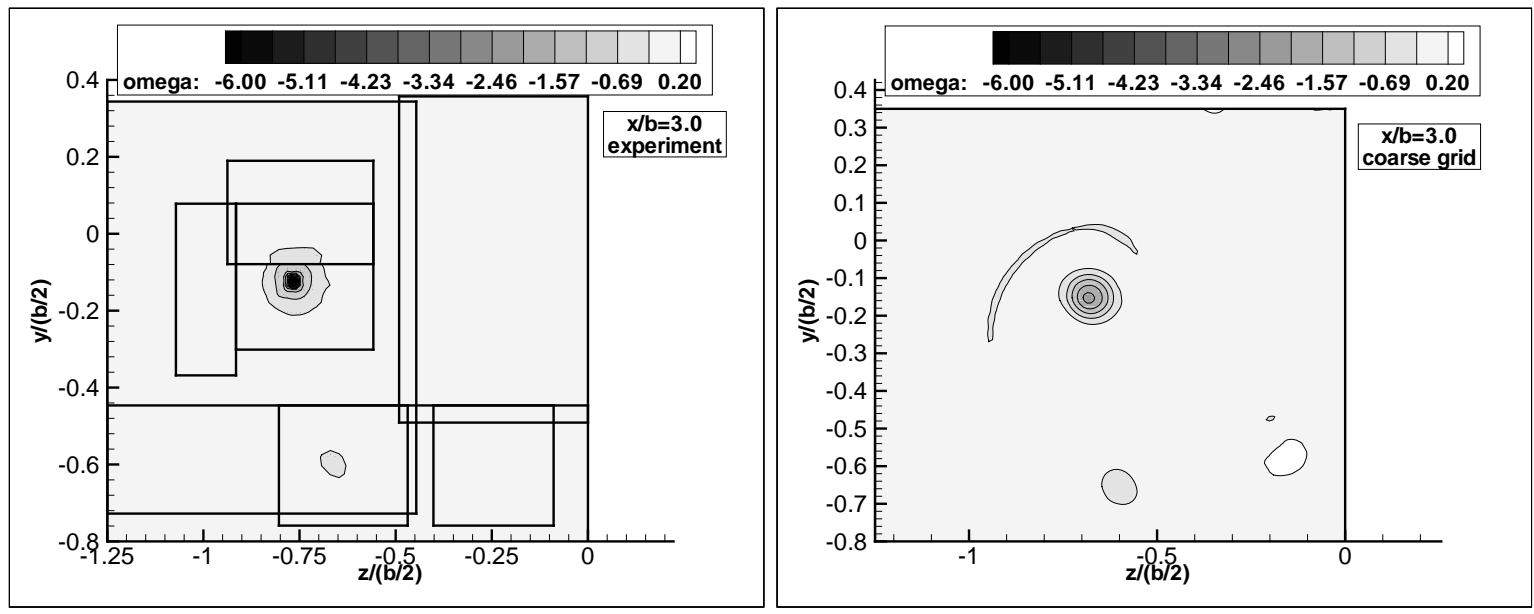

Figure 5.29: Experimental (left) and numerical (right) vorticity distribution of an A300 wake at $x / b=3.0$ for $M a_{\infty}=0.14$ and $R e_{c}=200000$
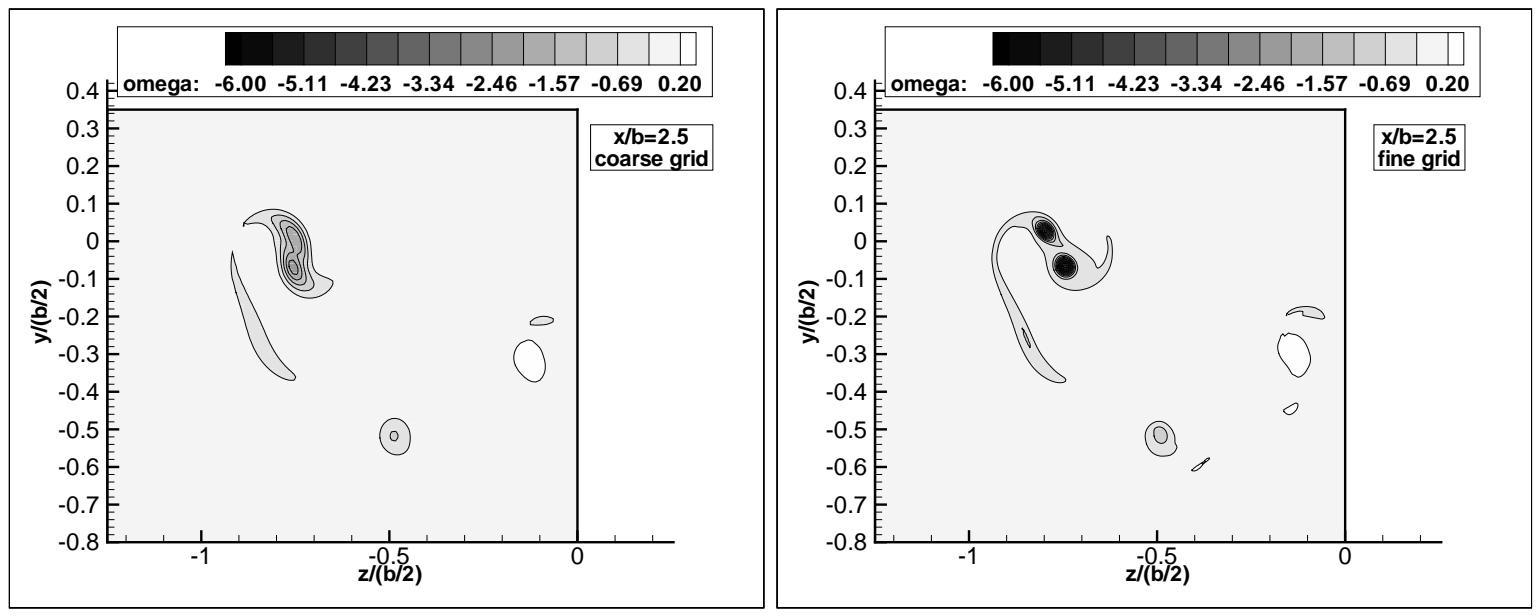

Figure 5.30: Numerical simulation on the coarse (left) and fine grid (right). Vorticity distribution of an A300 wake at $x / b=2.5$ for $M a_{\infty}=0.14$ and $R e_{c}=200000$ 

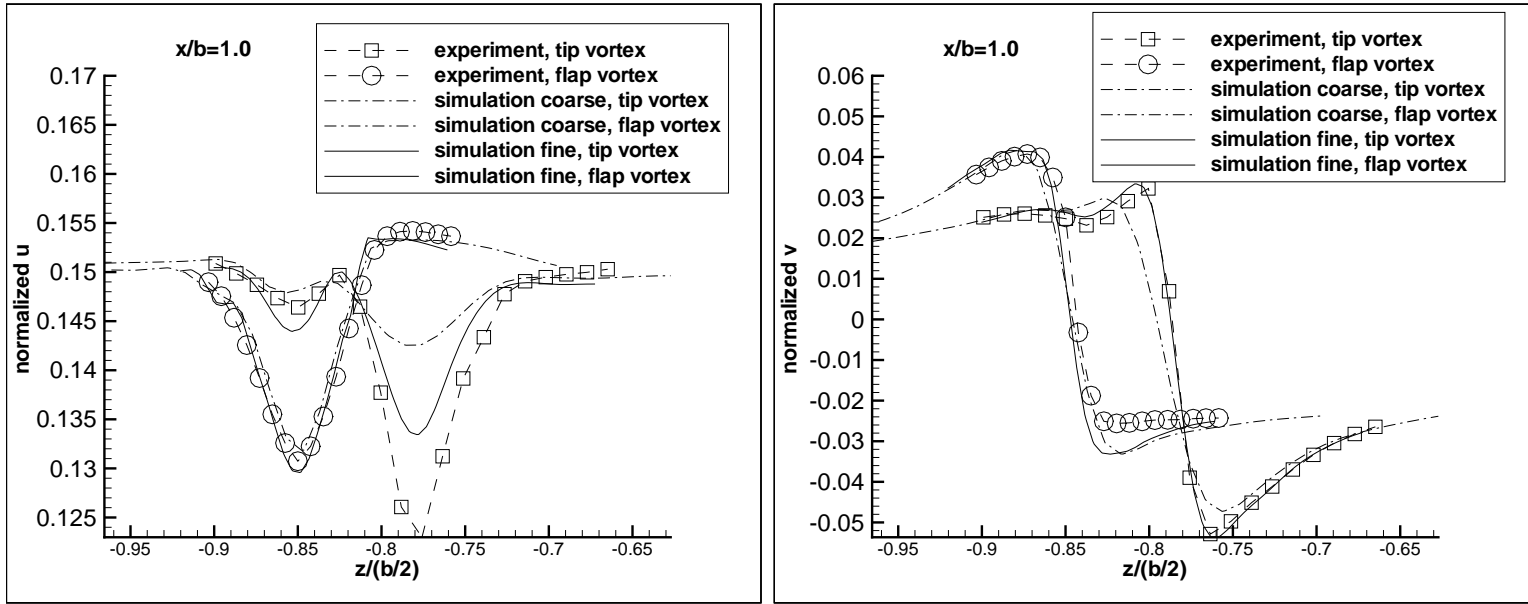

Figure 5.31: Comparison of the numerical and experimental distributions of the velocity components $u$ and $v$ of the wingtip and flap side-edge vortex of an A300 wake at $x / b=1.0$ for $M a_{\infty}=0.14$ and $R e_{c}=200000$

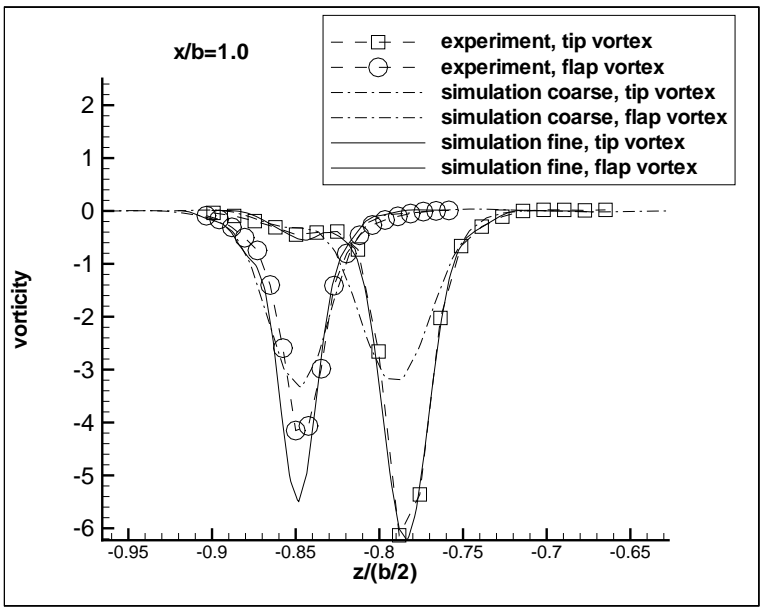

Figure 5.32: Comparison of the numerical and experimental distribution of the vorticity of the wingtip and flap side-edge vortex of an A300 wake at $x / b=1.0$ for $M a_{\infty}=0.14$ and $R e_{c}=200000$ 


\section{$5.2 \quad$ Rectangular Wing with Engine Jets}

The wingtip vortices usually have a core radius $r_{c}$ that is at least one order of magnitude smaller than the characteristic length of the generating wing, e.g. the chord length $L_{c}$, which in turn is one order of magnitude smaller than the wingspan $b$. A cartesian uniform grid in the wake resolving the wingtip vortex by only 10 grid points per core radius and having the dimensions $b \times L_{c} \times b$ will lead to a total number of grid points in the order of $\mathcal{O}\left(10^{8}\right)$ to adequately resolve the shear layer of the wing and the wake up to 1 span downstream of the wing. Multiblock curvilinear grids can reduce the total number of grid points but unfortunately cannot be generated without knowledge of the exact behavior of the wake since the wake roll-up and the interaction with the engine jet is not known a priori.

The flow around the wing including the engine nacelle and pylon on the other hand requires a body fitted stretched mesh of $\mathcal{O}\left(10^{6}\right)$ grid points to adequately resolve the turbulent boundary layer and to simulate the jet flow. If both the wake and the wing flow are calculated simultaneously using only one multiblock structured mesh then a total of $\mathcal{O}\left(10^{9}\right)$ equations must be solved to achieve a correct resolution of the flow field that covers the wing and the wake region no more than just one wingspan downstream of the wing's trailing edge. Since such a calculation with the necessary computational resources would be far too expensive a different approach is proposed.

The numerical simulation is divided into two steps. First, the flow field around the wing is determined and subsequently, the data of this simulation is used to provide the inflow condition for a separate wake simulation. This approach has been used previously in similar studies $[25,26]$ and proved to be very successful. The only assumption is that in the first step the unresolved wake does not have a relevant influence on the flow behavior near the wing and the lift generation. This is justified according to the assumption that the induced velocities from the wake on the wing and therefore the locally induced angle of attack are small compared to the geometrical angle of attack [105], which means that if they are underestimated due to the somewhat underresolved vortex sheet in the wake the lift generation would still be adequate. A similar two step approach is also proposed by STUMPF [121] using EULER computations on unstructured grids for the lift generating wing flow followed by unsteady wake calculations on cartesian grids of the viscous wake flow.

Other authors [29, 72] reduce the equations even further. Following investigations by Rosenhead [91] and Westwater [135] they assume that the wake flow can be parabolized and may be reformulated as an unsteady $2 \mathrm{D}$ problem. This simplification implies a constant dominating flow velocity $u$ in the main flow direction and neglects several physical phenomena like vortex stretching, vortex line curvature as well as all gradients in the main flow direction. Such a parabolic approach can be an appropriate approximation of the wake flow up to 70 wingspans behind the wing as long as the assumptions are fulfilled. However, the jet flow, which is also part of this analysis, contradicts the major assumption that the main stream velocity is constant. Therefore, the parabolized formulation is not applicable in this analysis.

According to the aforementioned two step numerical approach the investigation in the 
following sections is divided into two parts. First, the flow around the rectangular wing is discussed and subsequently, the wake flow is investigated.

\subsubsection{Flow Around the Rectangular Wing}

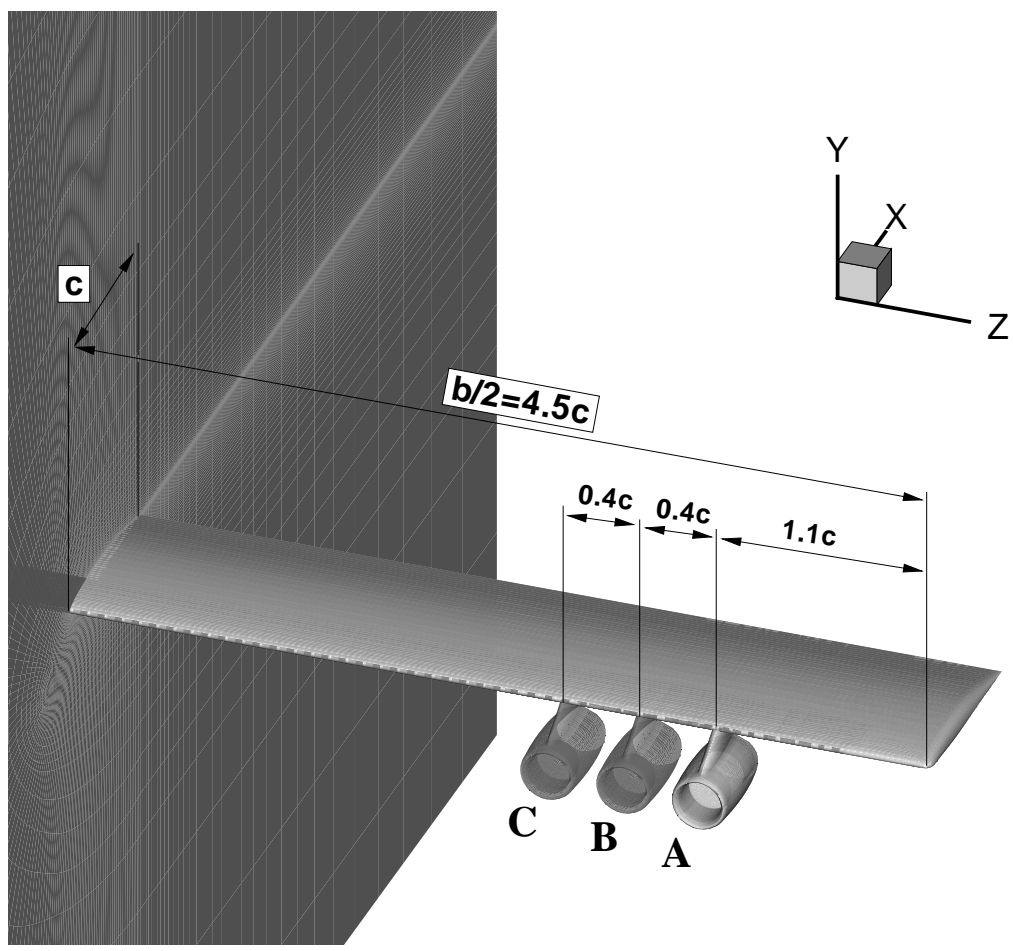

Figure 5.33: Geometry, coordinate system and positions of the engine

The wing/engine configuration is defined in the collaborative research program SF B 401 at the RWTH AACHEN. The aspect ratio is typical for future large aircraft. The rectangular wing has an aspect ratio of $\Lambda=9.0$ and a $B A C 3-11$ profile [65]. The engine geometry is similar to the Trent 900 engine. In the experimental investigations three different positions of the model engine can be chosen, each $0.4 c$ apart, with a minimum distance of $1.1 c$ from the wingtip (figure 5.33). The positions are numbered $\mathbf{A}, \mathbf{B}$ and $\mathbf{C}$, where $\mathbf{A}$ is nearest the wingtip.

The structured multiblock grid used for the simulation of the wing and the engine flow is shown in figure 5.34. C-type and O-type grid topologies were used for the wing in the streamwise and the spanwise direction, respectively. The blunt trailing edge of the wing allows to use this topology without generating grid singularities. The pylon was resolved using a C-type mesh and an O-type topology was used for the nacelle of the engine. This topology was deliberately chosen to circumvent small cells at intersecting surfaces such as in the pylon/wing case. Furthermore, the final grid consists of different blocks that can be arranged to reposition the engine in the spanwise direction without a complete regeneration of the grid. Due to the analysis in chapter 5.1.3 the grid spacing near the surface is chosen such that the first grid point lies at $y^{+}=1$. To approximate this distance for a specific REYNOLDS number a turbulent incompressible flat 


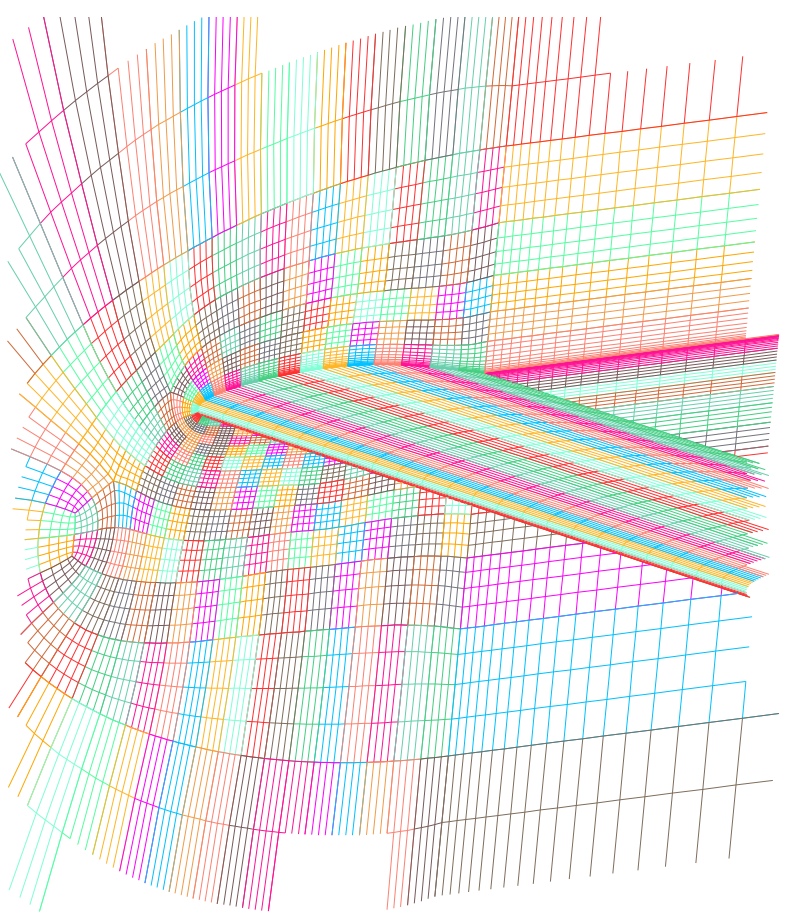

Surface grid at wing root

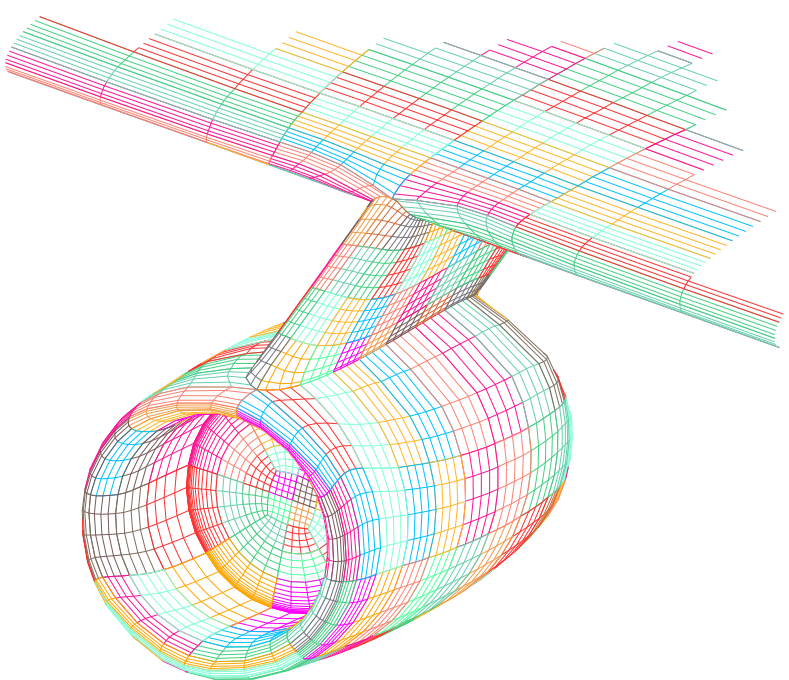

Wing, pylon and nacelle surface grid showing engine inflow plane

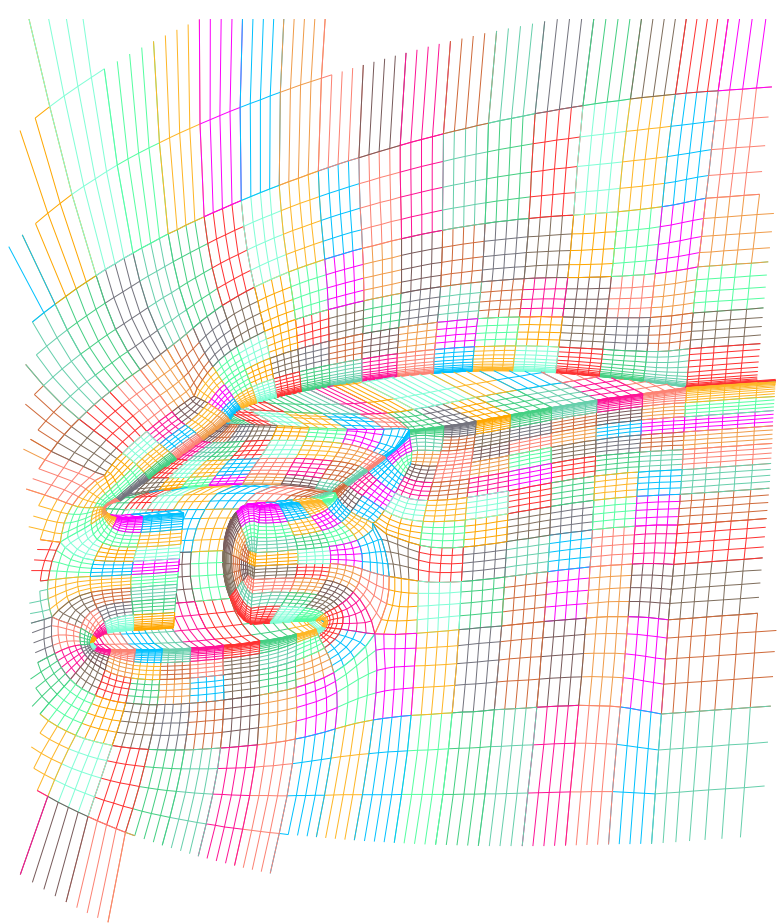

Cut through nacelle, pylon and wing

Figure 5.34: Computational grid, 74 blocks, 1.1 million nodes 
plate boundary layer is assumed with the following approximation for the skin-friction distribution [106]

$$
c_{f}=\frac{\tau_{w}}{\frac{1}{2} \rho u_{\infty}^{2}} \approx 0.0576 R e_{x}^{-1 / 5}
$$

Inserting equation (5.20) into the definition of $y^{+}(5.13)$ an approximation for $y / L$ can be achieved

$$
\frac{y}{L}=\frac{y^{+}}{R e_{L}} \sqrt{\frac{2}{c_{f}}} .
$$

Using $R e_{x}=R e_{L} \cdot x / L$ with the characteristic length $L$ being the chord length $c$ and $x / L=x / c=\mathcal{O}(1)$ being the normalized coordinate in the flow direction a minimum normal distance $y / c \approx 5.0 \cdot 10^{-5}$ is calculated for $y^{+}=1$ and $R e_{c}=268250$.

The flow simulation of the wing and wake flow is performed for six configurations that are listed in table 5.3. The table also includes a comparison of the calculated values of the force coefficients $c_{L}, c_{D}$ and $c_{Z}$. The flow parameters are chosen according to the experimental investigation in the subsonic wind tunnel that are performed separately within the $S F B 401$ research program [44]. The engine jet velocity ratio $u_{\text {jet }} / u_{\infty}$ in real configurations depend on many factors such as cruise conditions and bypass ratio. The simulated values can be compared to a landing configuration. The boundary layer of the wing is tripped at the leading edge to enforce a fully turbulent flow on the suction and pressure side. The numerical simulation was considered converged to the desired steady state if changes in the lift coefficient $c_{L}$ over 50 iterations are less than $1 \%$. An exemplary convergence history for the computation of the flow field over configuration pos $A$ is shown in figure 5.35 .

\begin{tabular}{|c|c|c|c|c|c|c|}
\hline Configuration & Engine Position & $\varepsilon_{\infty / \text { in }}$ & $u_{\text {jet }} / u_{\infty}$ & $c_{L} / c_{L_{\text {pos } A}}$ & $c_{D} / c_{D_{\text {pos } A}}$ & $c_{Z} / c_{Z_{\text {pos } A}}$ \\
\hline \hline pos $A$ & $\mathbf{A}$ & 1.7 & 1.77 & 1.00 & 1.00 & 1.00 \\
\hline pos $B$ & $\mathbf{B}$ & 1.7 & 1.77 & 1.01 & 0.99 & 0.99 \\
\hline pos $C$ & $\mathbf{C}$ & 1.7 & 1.77 & 0.99 & 0.97 & 0.91 \\
\hline pos $A-t 2$ & $\mathbf{A}$ & 2.5 & 2.87 & 1.00 & 0.91 & 0.94 \\
\hline posB-t2 & $\mathbf{B}$ & 2.5 & 2.87 & 1.00 & 0.90 & 0.95 \\
\hline pos $C$ - $t 2$ & C & 2.5 & 2.87 & 0.98 & 0.87 & 0.88 \\
\hline \hline
\end{tabular}

characteristic values:

$$
M a_{\infty}=0.180265 \quad R e_{c}=268250 \quad \alpha=8^{\circ}
$$

further values for jet simulation:

$$
T_{\text {jet }} / T_{\infty}=1.0 \quad \check{\nu}_{j e t} / \nu=10.0 \quad \dot{m}_{f} / \dot{m}_{i n}=0.0
$$

values for turbulence model:

$$
\check{\nu}_{\text {init }} / \nu=0.1 \quad T u_{\text {init }}=0.01
$$

Table 5.3: Parameters of the simulated configurations and calculated force coefficients 


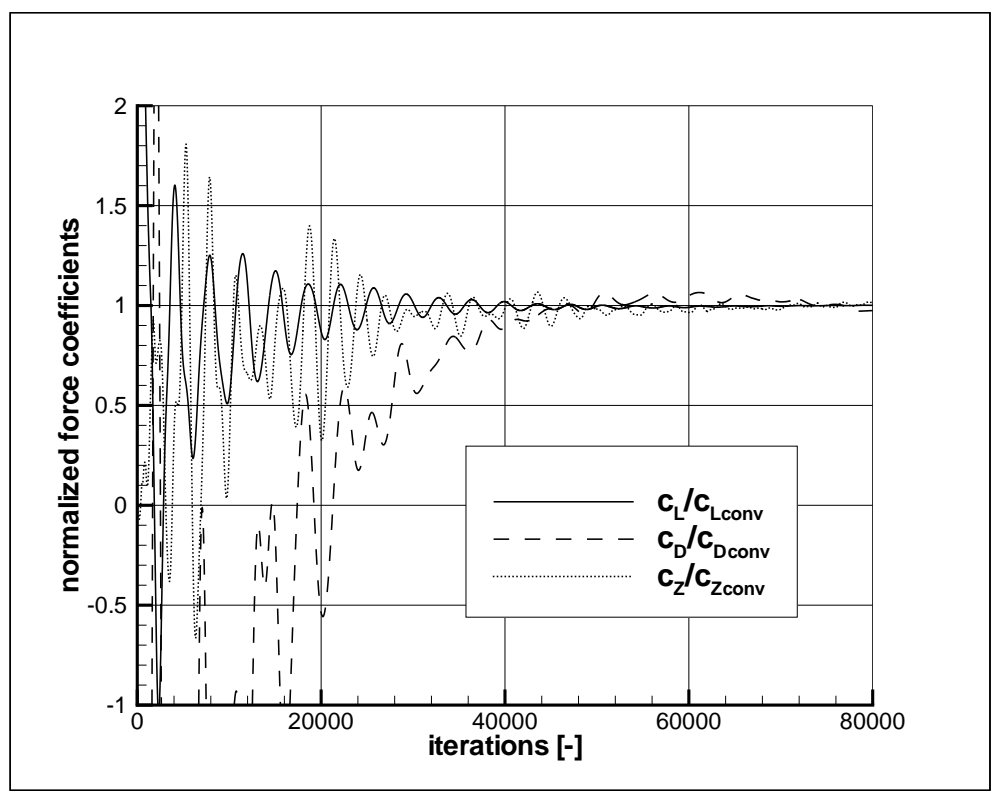

Figure 5.35: Convergence history of the force coefficients for configuration $\operatorname{pos} A$

For all six configurations only a minor change of less than $2 \%$ in the lift coefficient $c_{L}$ was determined. Thus an almost equal strength of the circulation $\Gamma$ of the wingtip vortex is obtained. On the other hand, a change of almost $13 \%$ of the drag coefficient $c_{D}$ was noticed. The smaller the distance between the engine and the wingtip, the higher the drag. This tendency, which also holds for the force coefficient $c_{Z}$ in the spanwise direction, was found at both jet velocities.

The pressure distribution on the wing/pylon/nacelle surfaces are presented for the six cases in figure 5.36. Qualitatively the pressure distribution on the wing is not affected by the presence of the engine or the jet velocity, since the vertical distance between the engine and the main wing is relatively large compared to current designs of modern civil aircraft. This is a consequence of the choice of the pylon geometry dictated by the experimental model.

\section{Engine Jet Behavior}

The jet flow shows differences in all cases studied as presented in figure 5.37. The engine jet with the higher velocity $u_{\text {engine }} / u_{\infty}=2.87$ shows almost similar behavior for all three engine positions posA-t2, posB-t2 and posC-t2. The direction of the jet is hardly influenced by the position of the engine whereas the core size shows a slight tendency to get more slender with growing distance to the wingtip. The engine jet with the lower velocity $u_{\text {engine }} / u_{\infty}=1.77$ on the other hand shows a different behavior with the tendency of the jet to get wider with growing distance to the wingtip. The direction of the jet is similar for all configurations with a slightly stronger downward deflection for the most outboard position. This can be explained by the induction influence of the wingtip vortex, since the jet in this configuration is nearest the wingtip. Note that 


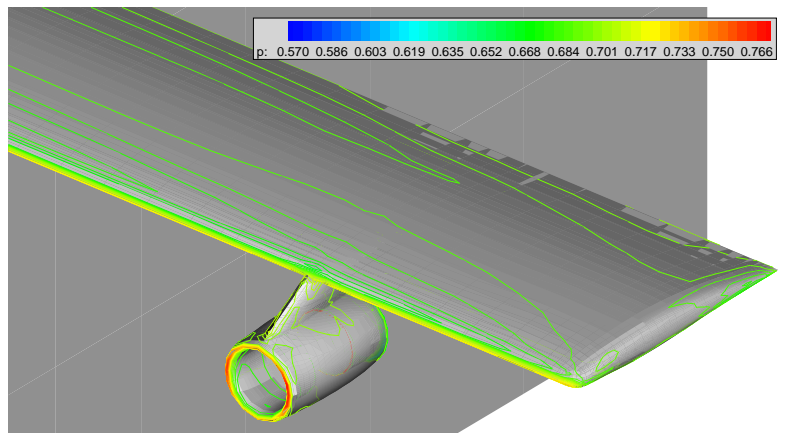

Engine position A $\quad u_{\text {engine }} / u_{\infty}=1.77$

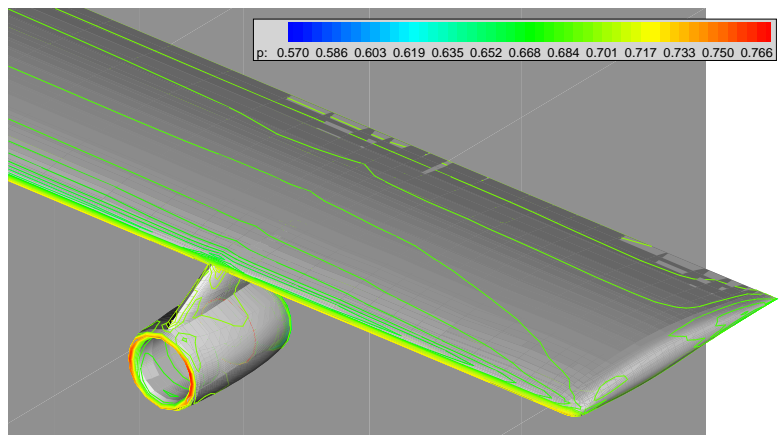

Engine position B $\quad u_{\text {engine }} / u_{\infty}=1.77$

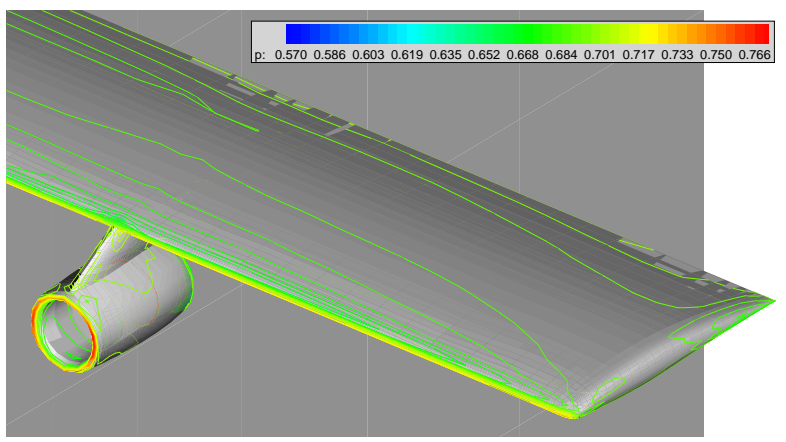

Engine position $\mathrm{C} \quad u_{\text {engine }} / u_{\infty}=1.77$

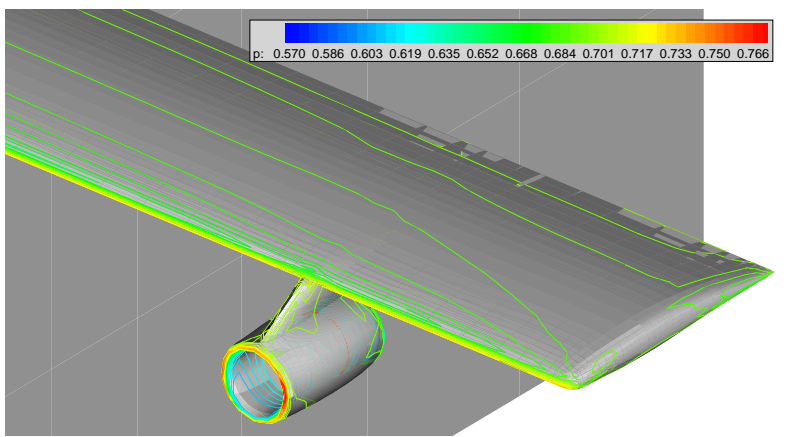

Engine position $\mathrm{A} \quad u_{\text {engine }} / u_{\infty}=2.87$

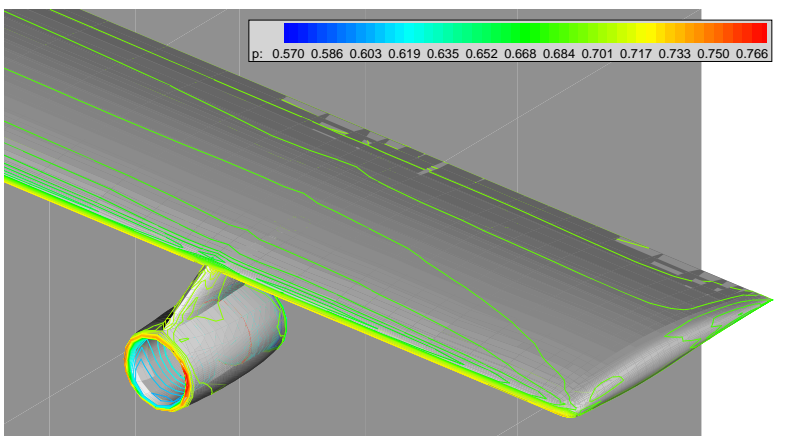

Engine position B $\quad u_{\text {engine }} / u_{\infty}=2.87$

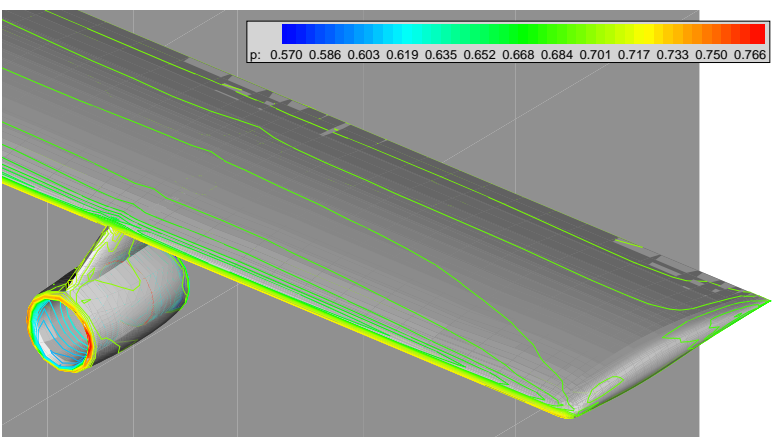

Engine position $\mathrm{C} \quad u_{\text {engine }} / u_{\infty}=2.87$

Figure 5.36: Pressure contours for $M a_{\infty}=0.18, R e_{c}=268250$ and $\alpha=8.0^{\circ}$ 
this influence is missing in the configuration with the higher jet velocity posA-t2. It is therefore concluded that the influence of the wingtip in that stage of the flow is limited only to the nearest position pos $A$ with the low jet velocity. Note also that the jet velocity $u_{\text {engine }} / u_{\infty}=1.77$ for the configurations pos $A$, pos $B$ and pos $C$ is of the same order of magnitude as the excess velocity on the wing. It is therefore conjectured that the pressure conditions on the suction and pressure side of the wing play a stronger role in the shape and direction of the engine jet in these configurations than the ones with the higher jet velocities.

Quantitative $u$ velocity profiles in figure 5.38 give details about the small reduction of the maximum jet velocity according to its position. A deformation of the engine jet and a movement of its position is documented only at the lower engine jet velocity. This result confirms the aforementioned qualitative discussion that the engine jet with the lower velocity is generally more sensitive to wingtip vortex induction and to pressure distribution effects than the engine jet with the higher velocity. Nevertheless, the deformation of the engine jet shape itself is not a consequence of the induction of the wingtip vortex, since the jet nearest the wingtip vortex does not experience the strongest deformation as would be expected in such a case. This behavior is conjectured to be determined by the engine position with a specific pressure distribution on the pressure side of the wing.

\section{Wingtip Vortex Formation}

The generation of the wingtip vortex is documented in figures 5.39 and 5.40. The contours of the absolute value of the cross flow velocity component $\sqrt{v^{2}+w^{2}}$ and the streamwise velocity component $u$ show that the generation of the wingtip vortex is qualitatively similar in all cases. Quantitative differences can only be found in the cases pos $A$ and posA-t2, where the engine jet is positioned nearest the wingtip. This quantitative result is confirmed in figure 5.41 showing velocity profiles across the vortex core. Whereas the distribution is almost independent of the jet velocity, the position of the engine has a major impact on the wingtip vortex generation. This interaction is probably a consequence of the entrainment/detrainment effect of the jet, which reduces the velocity deficit of $u$ in the case of the engine jet next to the wingtip vortex. The asymmetric velocity distribution $\sqrt{v^{2}+w^{2}}$ is caused by the existence of the wake of the trailing edge. Again, the more apparent difference occurs for the configurations pos $A$ and $\operatorname{pos} A-t 2$. 


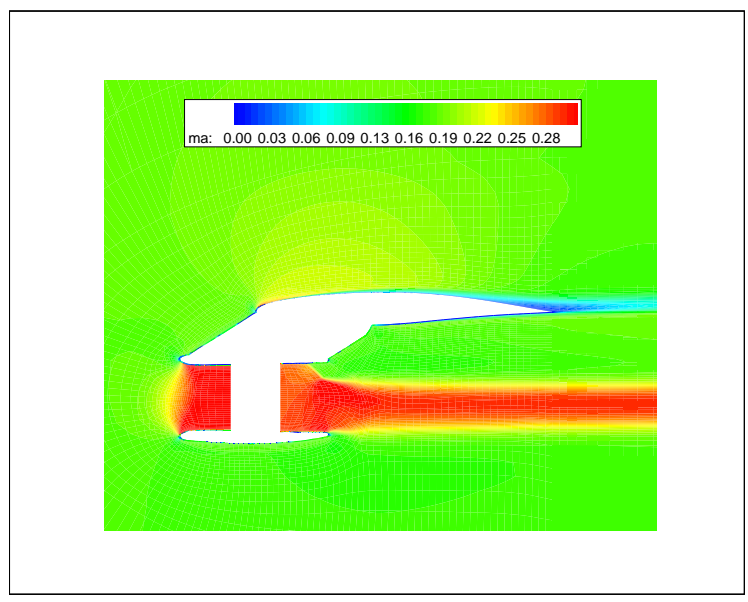

Engine position A $\quad u_{\text {engine }} / u_{\infty}=1.77$

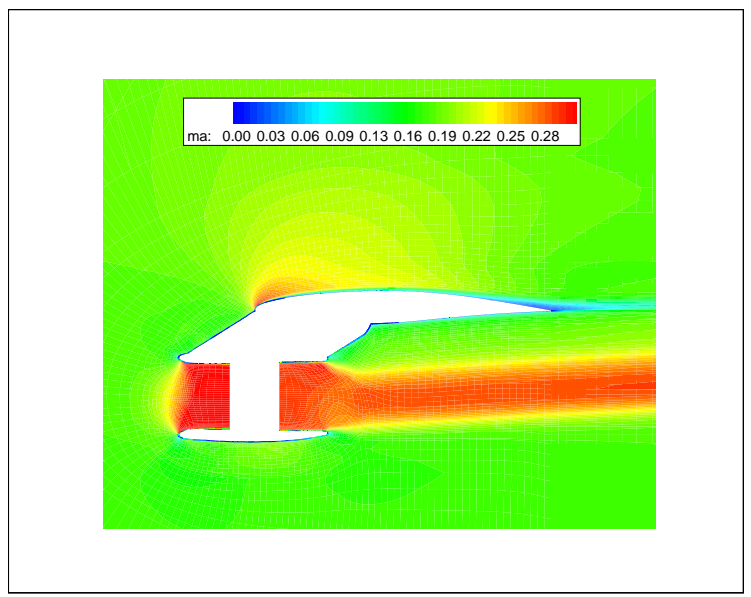

Engine position B $\quad u_{\text {engine }} / u_{\infty}=1.77$

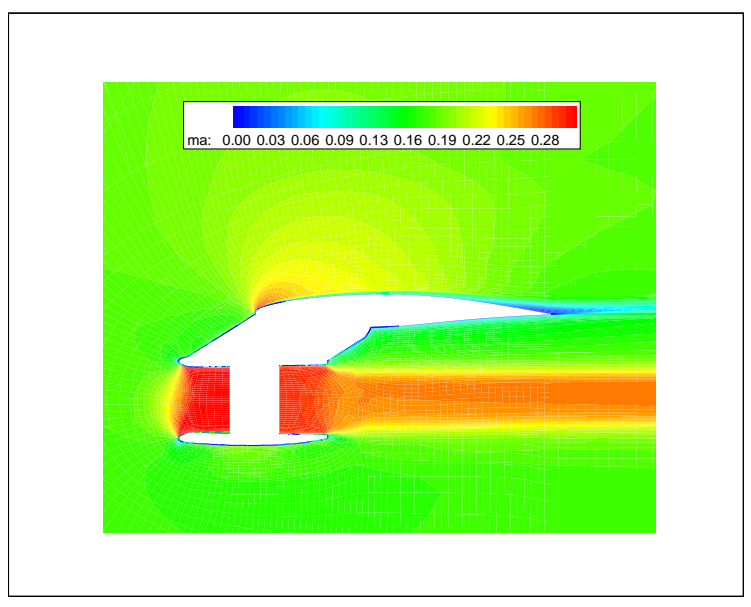

Engine position $\mathrm{C} \quad u_{\text {engine }} / u_{\infty}=1.77$

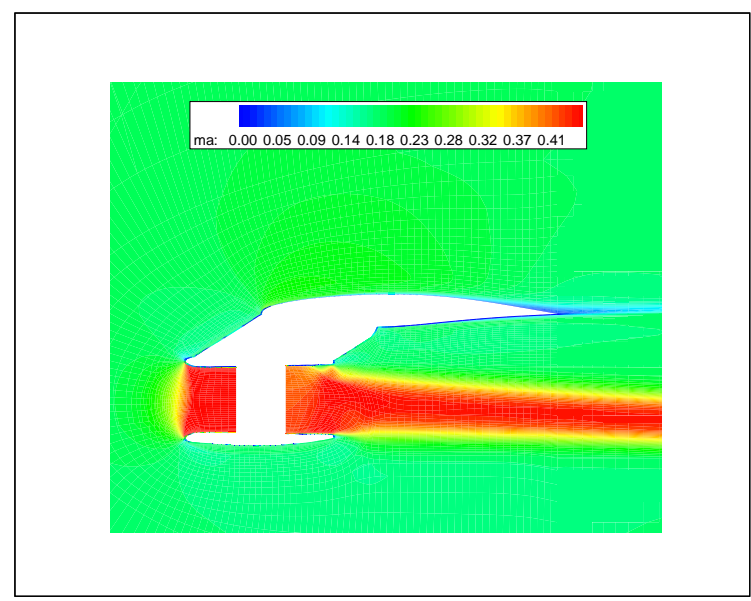

Engine position A $\quad u_{\text {engine }} / u_{\infty}=2.87$

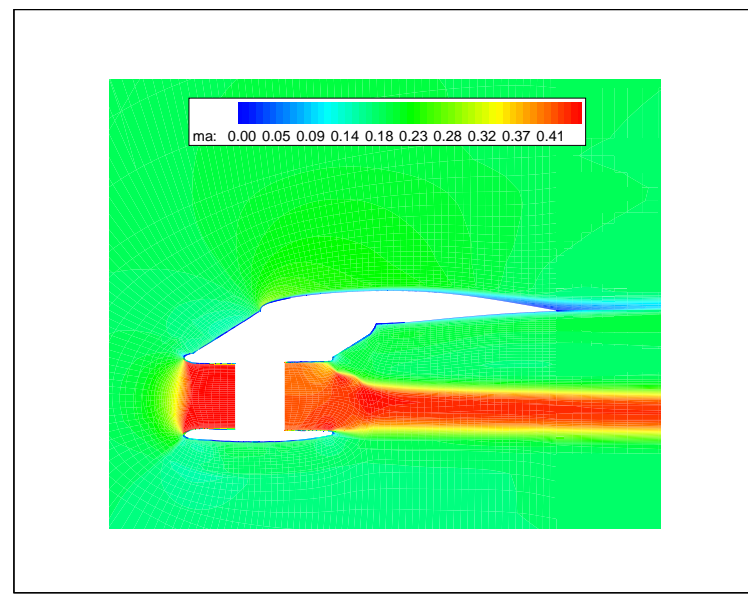

Engine position B $\quad u_{\text {engine }} / u_{\infty}=2.87$

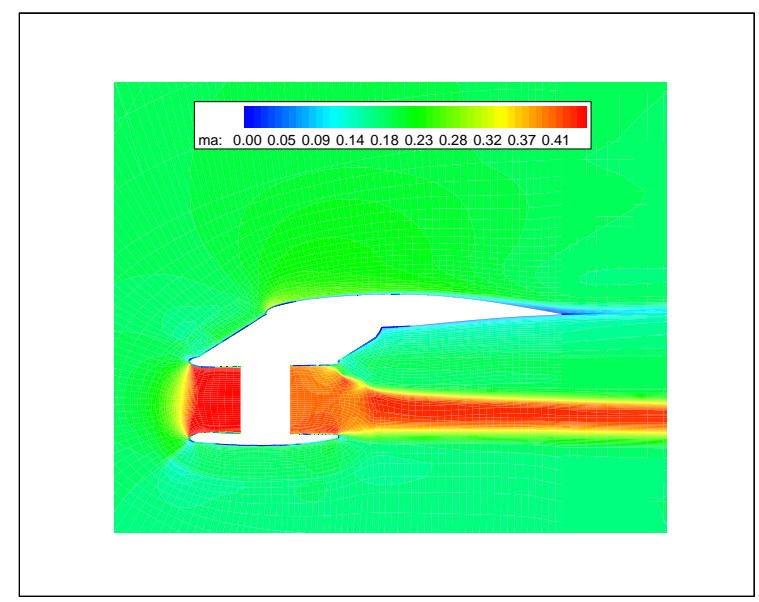

Engine position $\mathrm{C} \quad u_{\text {engine }} / u_{\infty}=2.87$

Figure 5.37: $\quad$ MACH number contours for $M a_{\infty}=0.18, R e_{c}=268250$ and $\alpha=8.0^{\circ}$ 


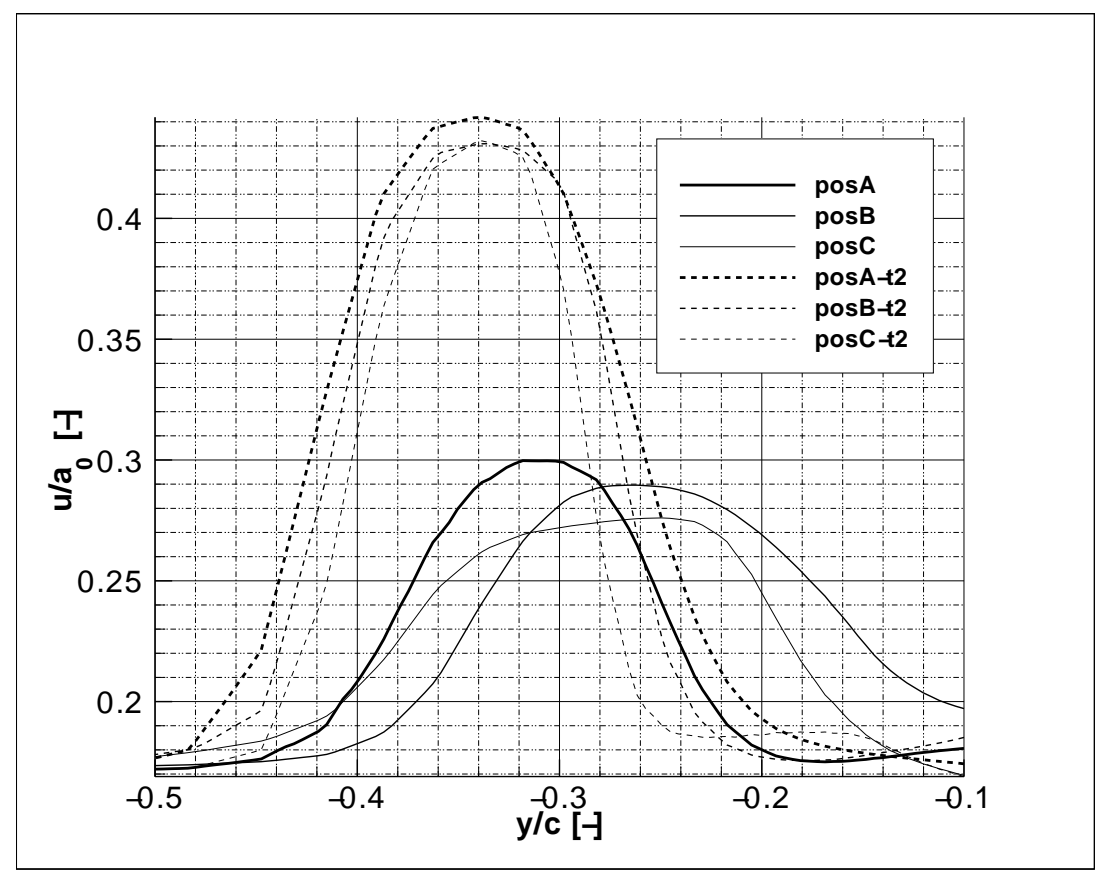

Figure 5.38: Comparison of the streamwise velocity $u$ profiles of the engine jet at $x / c=1.1$ for $M a_{\infty}=0.18, R e_{c}=268250$ and $\alpha=8.0^{\circ}$ 


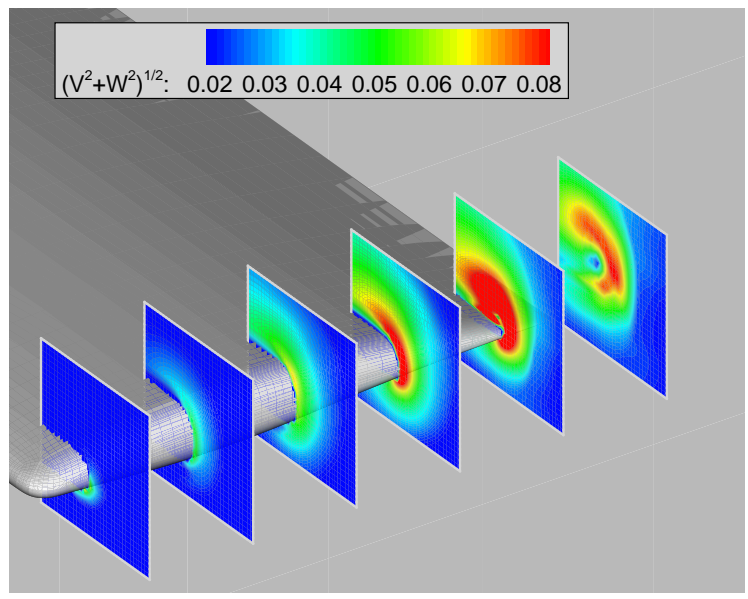

Engine position A $\quad u_{\text {engine }} / u_{\infty}=1.77$

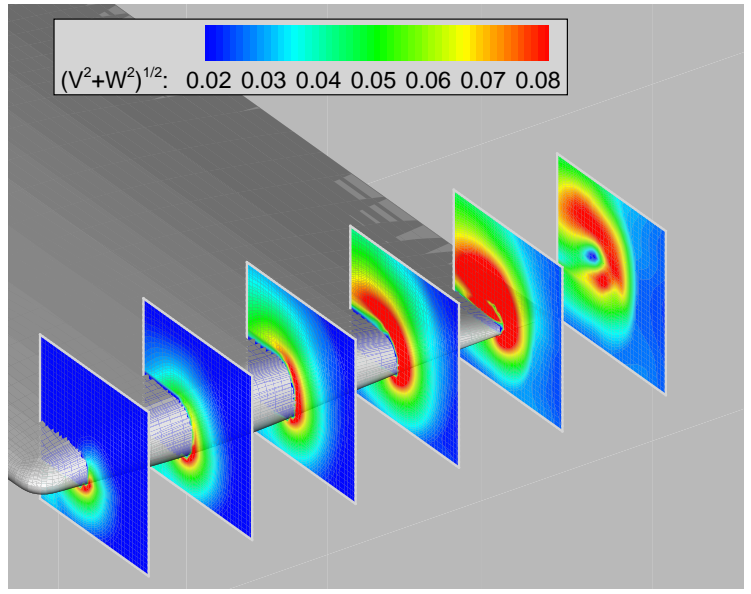

Engine position B $\quad u_{\text {engine }} / u_{\infty}=1.77$

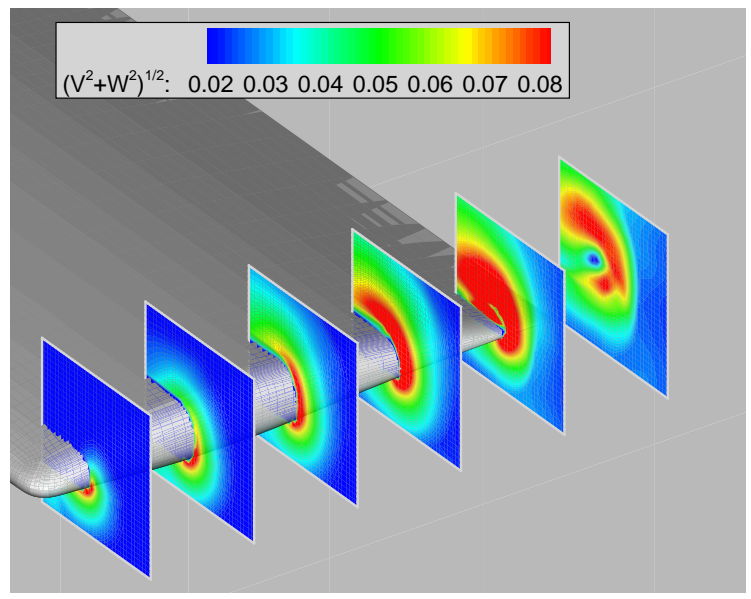

Engine position $\mathrm{C} \quad u_{\text {engine }} / u_{\infty}=1.77$

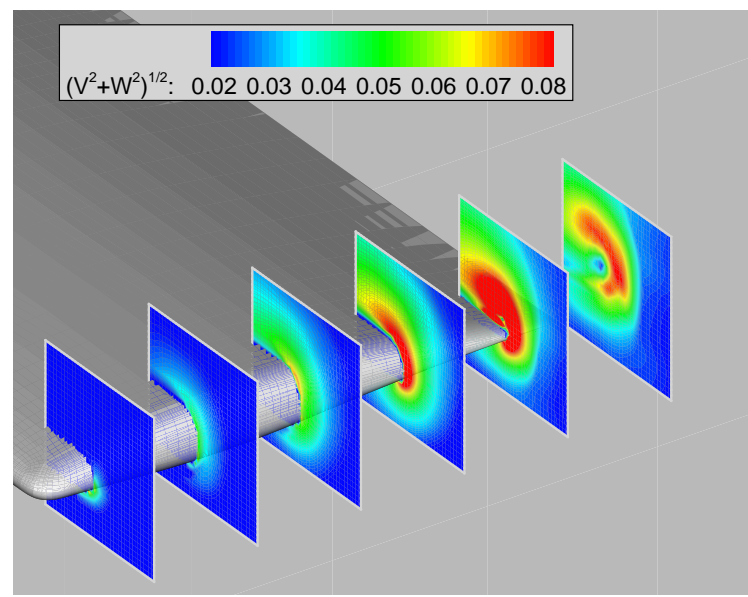

Engine position A $\quad u_{\text {engine }} / u_{\infty}=2.87$

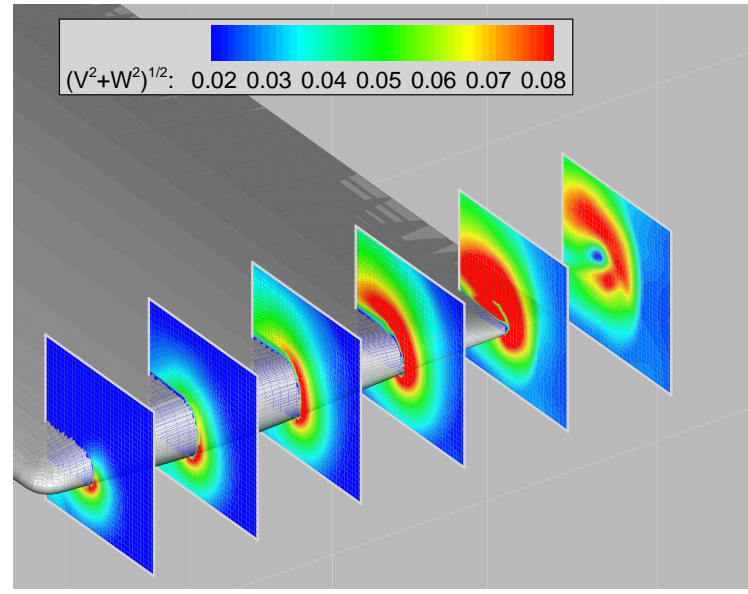

Engine position B $\quad u_{\text {engine }} / u_{\infty}=2.87$

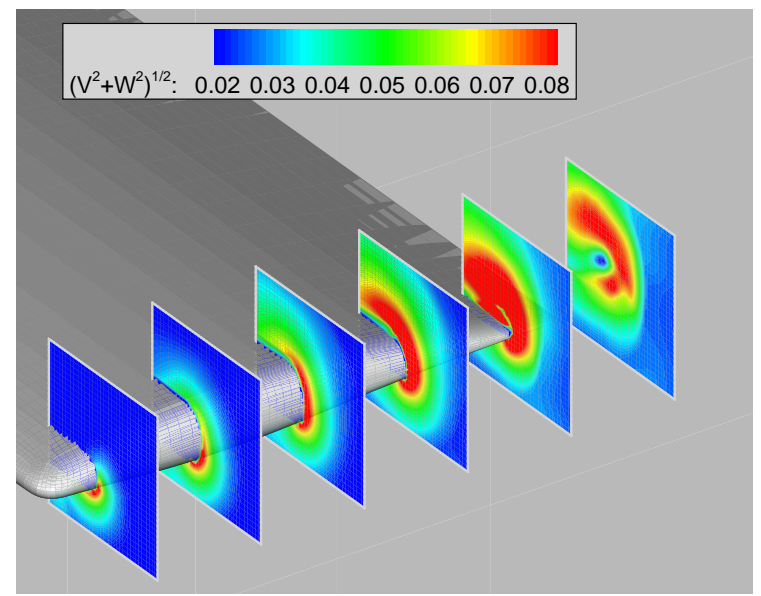

Engine position $\mathrm{C} \quad u_{\text {engine }} / u_{\infty}=2.87$

Figure 5.39: Velocity contours $\sqrt{v^{2}+w^{2}}$ at wingtip for $M a_{\infty}=0.18, R e_{c}=268250$ and $\alpha=8.0^{\circ}$ 


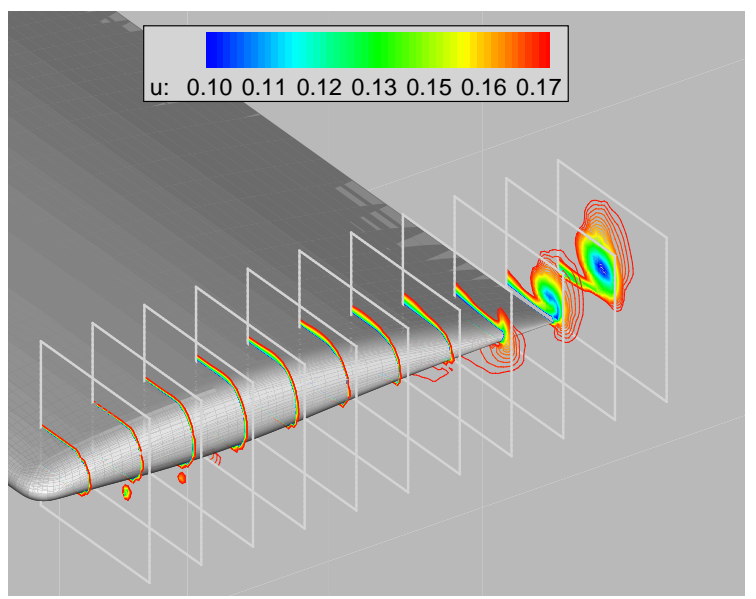

Engine position A $\quad u_{\text {engine }} / u_{\infty}=1.77$

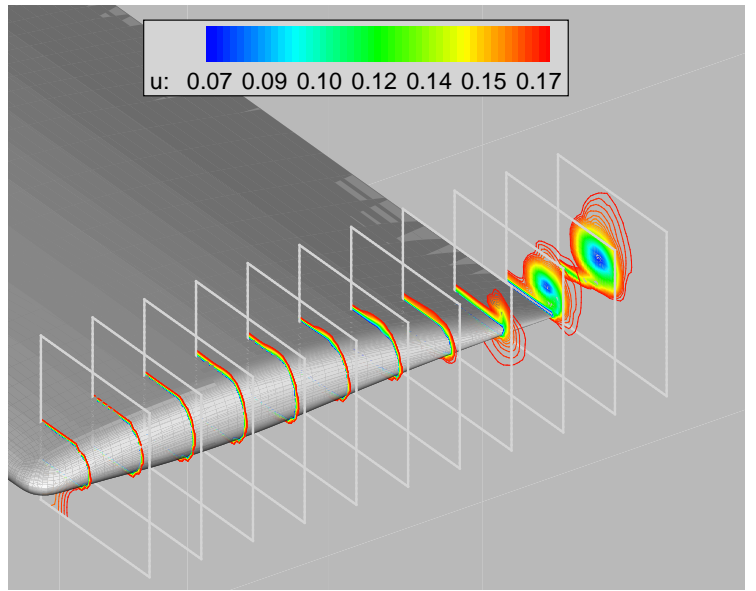

Engine position B $\quad u_{\text {engine }} / u_{\infty}=1.77$

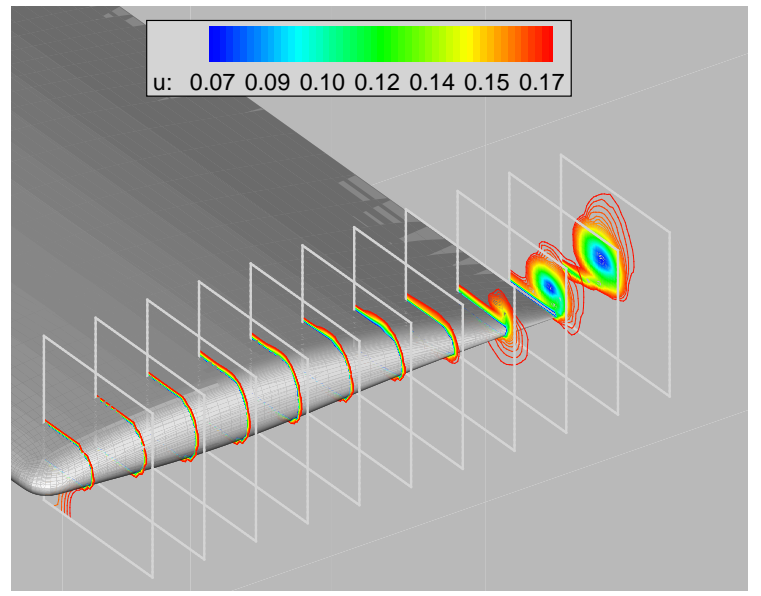

Engine position $\mathrm{C} \quad u_{\text {engine }} / u_{\infty}=1.77$

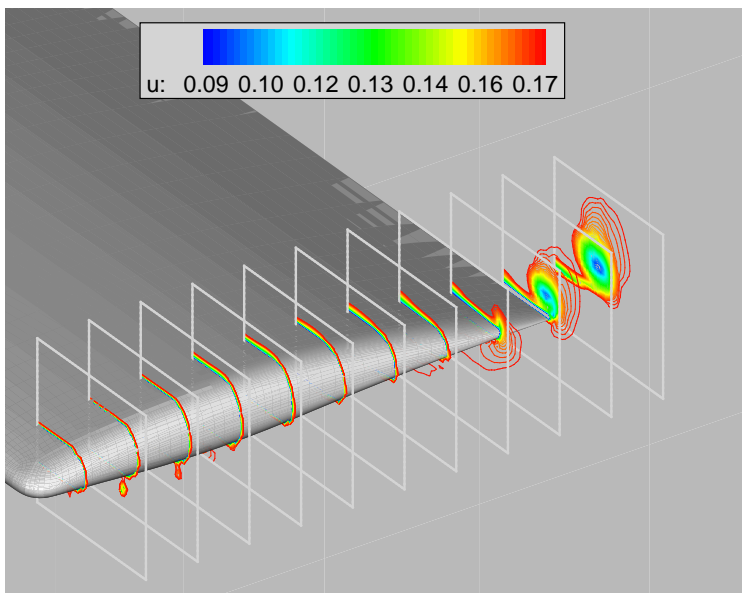

Engine position A $\quad u_{\text {engine }} / u_{\infty}=2.87$

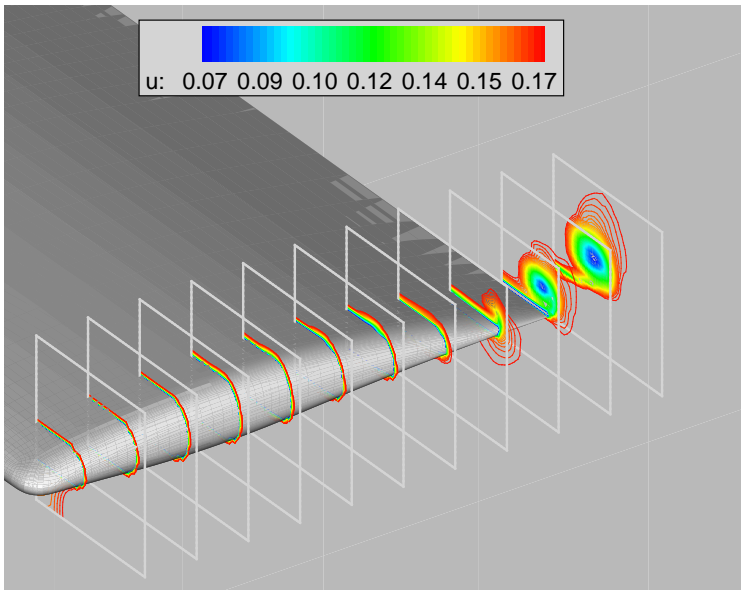

Engine position B $\quad u_{\text {engine }} / u_{\infty}=2.87$

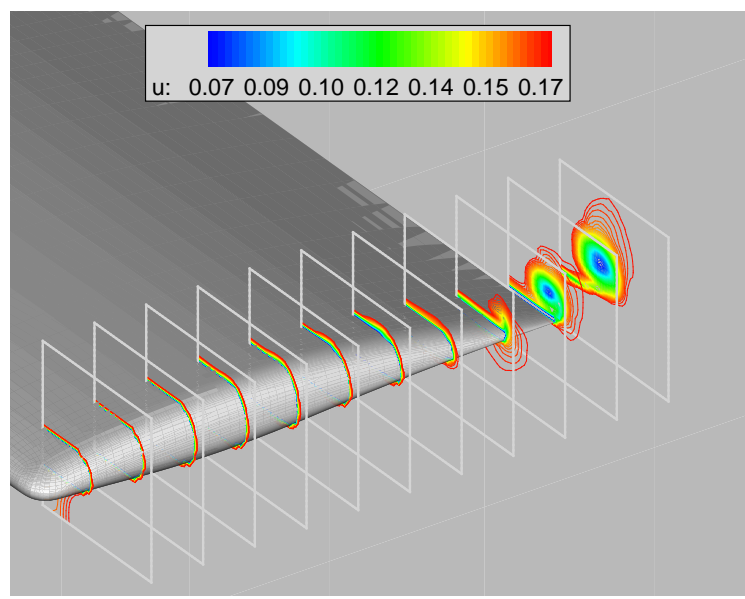

Engine position $\mathrm{C} \quad u_{\text {engine }} / u_{\infty}=2.87$

Figure 5.40: Contours of the streamwise velocity component $u$ at wingtip for $M a_{\infty}=0.18$, $R e_{c}=268250$ and $\alpha=8.0^{\circ}$ 

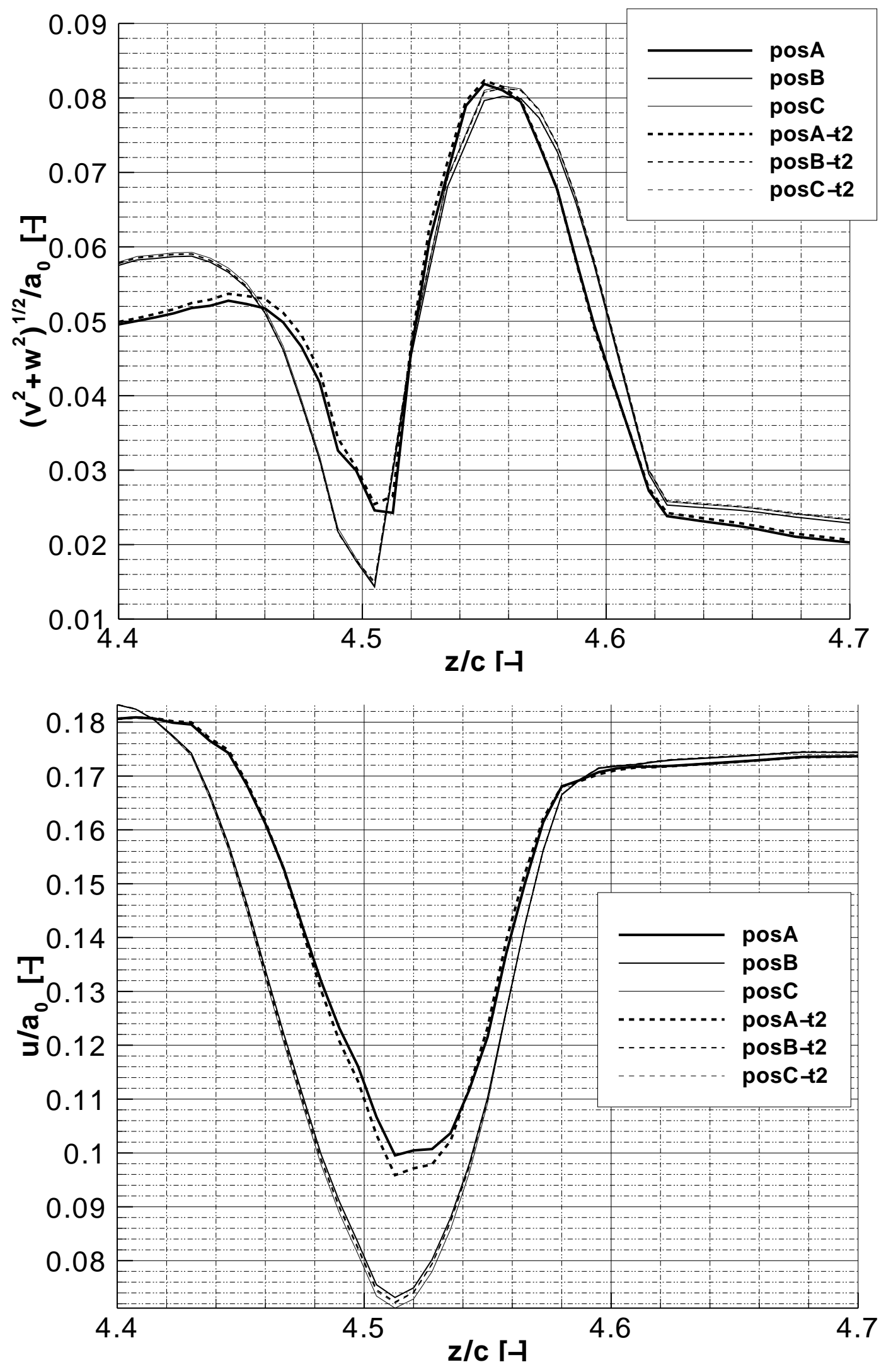

Figure 5.41: Comparison of the velocity profiles of the wingtip vortex at $x / c=1.1$ for $M a_{\infty}=0.18, R e_{c}=268250$ and $\alpha=8.0^{\circ}$ 


\subsubsection{Wake Flow}

The simulation of the wake was performed on a cartesian rectangular grid with $50 \times$ $334 \times 601$ grid points. This resulted in a resolution of the vortex by approximately 25 grid points in planes $x=$ const. The grid spacing in the main flow direction $\triangle x$ was 13 times larger than that in the other directions $\triangle y$ and $\triangle z$. The inflow variables were interpolated from the former calculation of the flow field over the wing in the plane $x / c=1.1$.

The three essential flow structures present in the wake are the shear layer, the wingtip vortex and the engine jet. Qualitative representations of these structures are presented using isosurfaces of vorticity and streamwise velocity in figures 5.42-5.53 for the six cases simulated.

\section{Shear Layer and Engine Jet Development}

The figures $5.42,5.44,5.46,5.48,5.50$ and 5.52 evidence the roll-up of the shear layer and thus the formation of the wingtip vortex. The shear layer undergoes a strong deformation especially near the engine jet. The engine jet, which does not have a circular shape in the inflow plane, also deforms continuously and interacts with the shear layer. The illustrations, e.g. figure 5.50, indicate through the wavy shape of the shear layer an unstable behavior. Just by looking at the figures it is evident that the strongest interaction is found in posB-t2 (figure 5.48) and the weakest in posB (figure 5.46). Hence, the essential difference is caused not by the different position but by a change in the jet velocity, which plays the major role. Comparison of the cases posB-t2 (figure 5.48) and posC-t2 (figure 5.52) exhibits that the instability of the shear layer and the engine jet is reduced for the case posC-t2 with the larger distance between the engine and the wingtip vortex. Recall from figures 5.41 and 5.38 that the engine and the wingtip have similar velocity profiles in the inflow plane $x / c=1.1$. That is, the pronounced differences do not occur due to the inlet distributions but are a consequence of differences in the spatial evolution of the flow, which is slightly influenced solely by the distance to the wingtip vortex.

It can be summarized that as far as the interaction between the shear layer and the engine jet is concerned the distance to the wingtip vortex plays a minor role. The engine jet velocity plays the major role, i.e. a stronger interaction between the engine jet and the shear layer occurs at higher engine jet velocities.

\section{Wingtip Vortex Development}

Isosurfaces of vorticity and streamwise velocity $u$ depicted in figures 5.43, 5.45, 5.47, $5.49,5.51$ and 5.53 show a tendency for a stronger deformation and disturbance of the engine jet for the cases with the higher jet velocity, whereas in all cases the engine jet has qualitatively almost no impact on the wingtip vortex. The distributions of cross-

flow velocity $\sqrt{v^{2}+w^{2}}$ and the streamwise velocity $u$ in figures 5.54-5.59 illustrate the development of the wingtip vortex for all cases at three locations downstream of the 
trailing edge $x / c=2.1,3.1,4.1$. At $x / c=2.1$ only small differences can be found between the various cases confirming former quantitative results shown in figure 5.41. In figures 5.55 and 5.58 at $x / c=3.1$ differences in the shape become more apparent between all cases, however, the position of the vortices, i.e. the position of the minimum value of $\sqrt{v^{2}+w^{2}}$ is almost the same in all cases. In the plane $x / c=4.1$ differences of the cross flow velocity $\sqrt{v^{2}+w^{2}}$ between all cases (figure 5.56) show the deformation, i.e. a clear deviation from the circular shape of the wingtip vortex is visible. This deformation is not apparent in the distributions of the streamwise velocity $u$ depicted in figure 5.59, since this velocity component is almost three times greater than the cross flow component $\sqrt{v^{2}+w^{2}}$. That is, the latter velocity component gives more details about the vortex core deformation, but the former reveals more information about the roll-up of the shear layer.

Note that the structure of the wake does not converge to a steady state. The aforementioned qualitative figures are considered to be snapshots of a time dependent flow. However, this does not prohibit a thorough analysis of the interaction between the wingtip vortices and the engine jets. As already mentioned before the wingtip vortex path is for example almost identical for all cases. The velocity distribution $\sqrt{v^{2}+w^{2}}$ exhibits deformations but is not essentially different, i.e. the wingtip vortex is not majorly influenced by the transient flow of the shear layer and engine jet within the computational domain. Further investigation of the detailed temporal development of these unsteady structures is beyond the scope of this work since such an analysis would require among other things a thorough, i.e. timely resolved, simulation of the turbulent structure. In other words, the approach would not be based on the REYNOLDS-averaged NAVIER-STOKES equations.

A quantitative investigation of the wingtip vortex is presented in figures 5.60-5.65. The vorticity profiles through the wingtip vortex show a similar quantitative development, even though the maximum vorticity for the cases pos $A$ and pos $A$-t2 at $x / c=2.1$ is somewhat lower than that of the more inboard other cases. Generally, the maximum vorticity is reduced in the following planes $x / c=3.1$ and $x / c=4.1$. At $x / c=4.1$ the maximum vorticity is comparable for all cases. The only exception is the case pos $B$, where a slight increase of the maximum vorticity from $x / c=3.1$ to $x / c=4.1$ is documented. Recall, that this configuration is also the one with the weakest shear layer instability and engine jet interaction (figure 5.46). Overall it can be concluded that the maximum vorticity at $x / c=4.1$ is independent of the engine position and the jet velocity. This is not the case for the streamwise velocity component $u$. Its distribution evidences that the wingtip vortex is strongly effected by the engine jet within the computational domain for the cases pos $A$ and posA-t2, whereas the more inboard positions possess distributions that are similar to each other but clearly different from those of pos $A$. The cases posA and posA-t2 represent the nearest position of the engine jet to the wingtip vortex and hence it is conjectured that this behavior is a consequence of the interaction with the engine jet, which is generated by the entrainment/detrainment effect of the jet. Due to the near field where the vortices are still in the process of rolling up the velocity deficit $u-u_{\infty}$ within the vortex core increases when the vortex moves downstream. Comparing the velocity profiles at $x / c=4.1$ it can be seen that 
this growth is weakest for the configurations pos $A$ and pos $A$-t2. Furthermore, from figures 5.64 and 5.65 it can be stated that the engine jet velocity plays only a minor role in this context.

According to mass conservation for an incompressible axisymmetric vortex [106]

$$
\frac{\partial u}{\partial x}+\frac{1}{r} \frac{\partial\left(r u_{r}\right)}{\partial r}=0
$$

a change of the axial velocity $\partial u / \partial x$ results in a change in the radial velocity component $u_{r}$ and consequently in a modification of the vortex core radius [72]. To satisfy mass conservation the axial velocity deficit $u-u_{\infty}$ leads to a negative radial velocity component $u_{r}$ in the vortex region, which compensates the streamwise velocity difference in the vortex core. Therefore, it can be argued that the smaller velocity deficit in the two cases with the engine jet nearest the wingtip can be offset in a much smaller distance downstream from the wing than for the configurations with the more inboard located engine and hence a tendency to reduce the wake hazard in the wake far field. Even though the findings are only valid in the near wake, the tendency can be extended to the far wake, since the numerical results show that the velocity deficit within the core is decreased as a consequence of the engine jet position near the wingtip throughout the whole computational domain and independent of the engine jet velocity. This tendency is expected to be also valid for the far wake.

It can be summarized that if the wingtip vortex is characterized by the distribution of the azimuthal velocity distributions it is not strongly influenced by the engine jet and retains most of its structure and strength throughout the computational domain. Nevertheless, the velocity deficit within the vortex core for the streamwise velocity $u$ distribution across the vortex evidences the interaction between the wingtip vortex and the engine jet.

\section{Summary of the Influence of the Parameters Engine Jet Velocity and Engine Position}

The higher engine jet velocity generally amplifies the instabilities of the inboard shear layer in the wake flow. No pronounced difference of the two engine jet velocities simulated on the formation and development of the wingtip vortex was found.

The distance between the engine jet and the wingtip vortex in the spanwise direction is important in the formation and development of the wingtip vortex and to a limited extent in the deformation of the engine jet. It was found that the velocity deficit within the wingtip vortex core is reduced for the engine position closest to the wingtip, whereas the trajectory of the vortex core of the vorticity maximum is only slightly

effected by the engine position. 


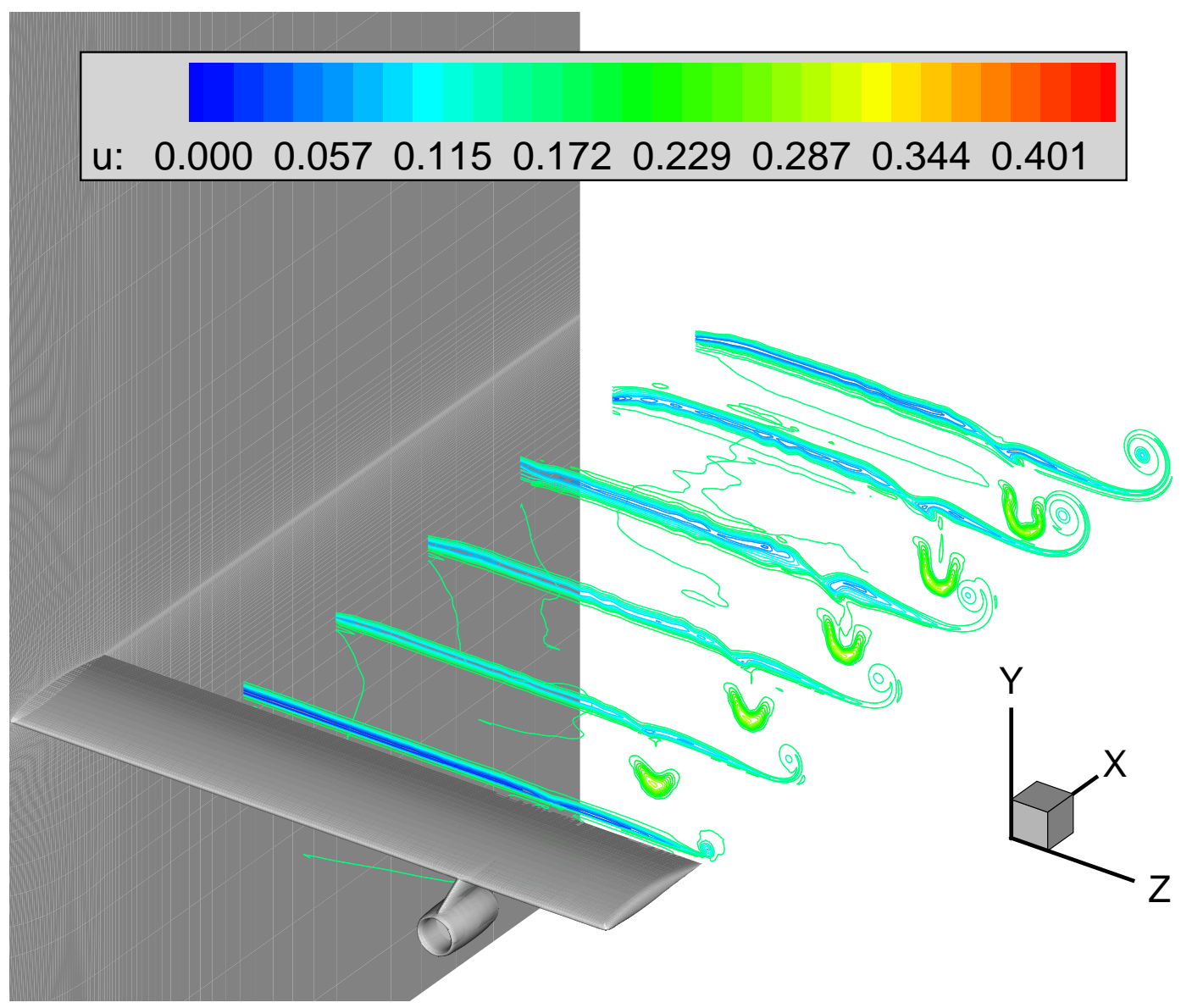

Figure 5.42: Contours of the streamwise velocity $u$ in the wake at $x / c=$ const. for pos $A$, $u_{\text {engine }} / u_{\infty}=1.77$

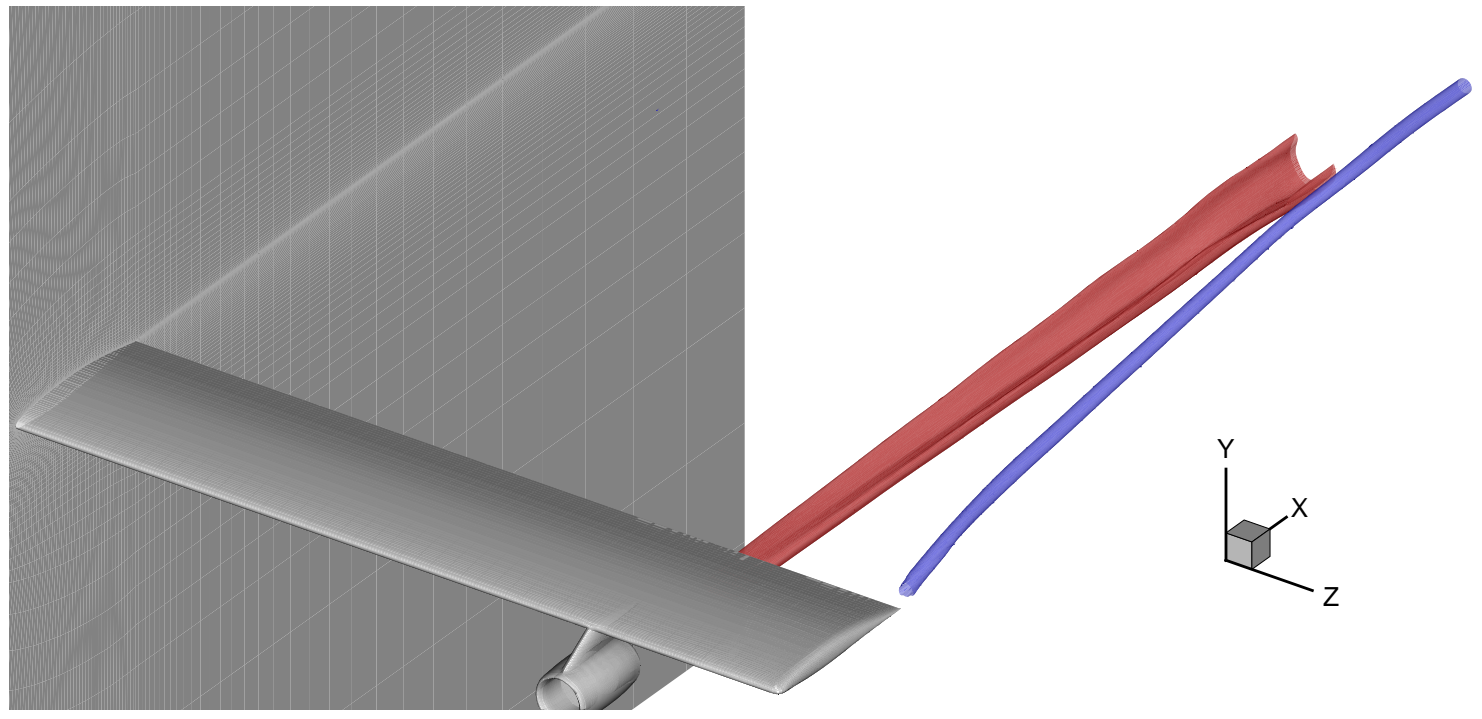

Figure 5.43: Isosurfaces of vorticity and streamwise velocity $u$ for $p o s A, u_{\text {engine }} / u_{\infty}=1.77$ 


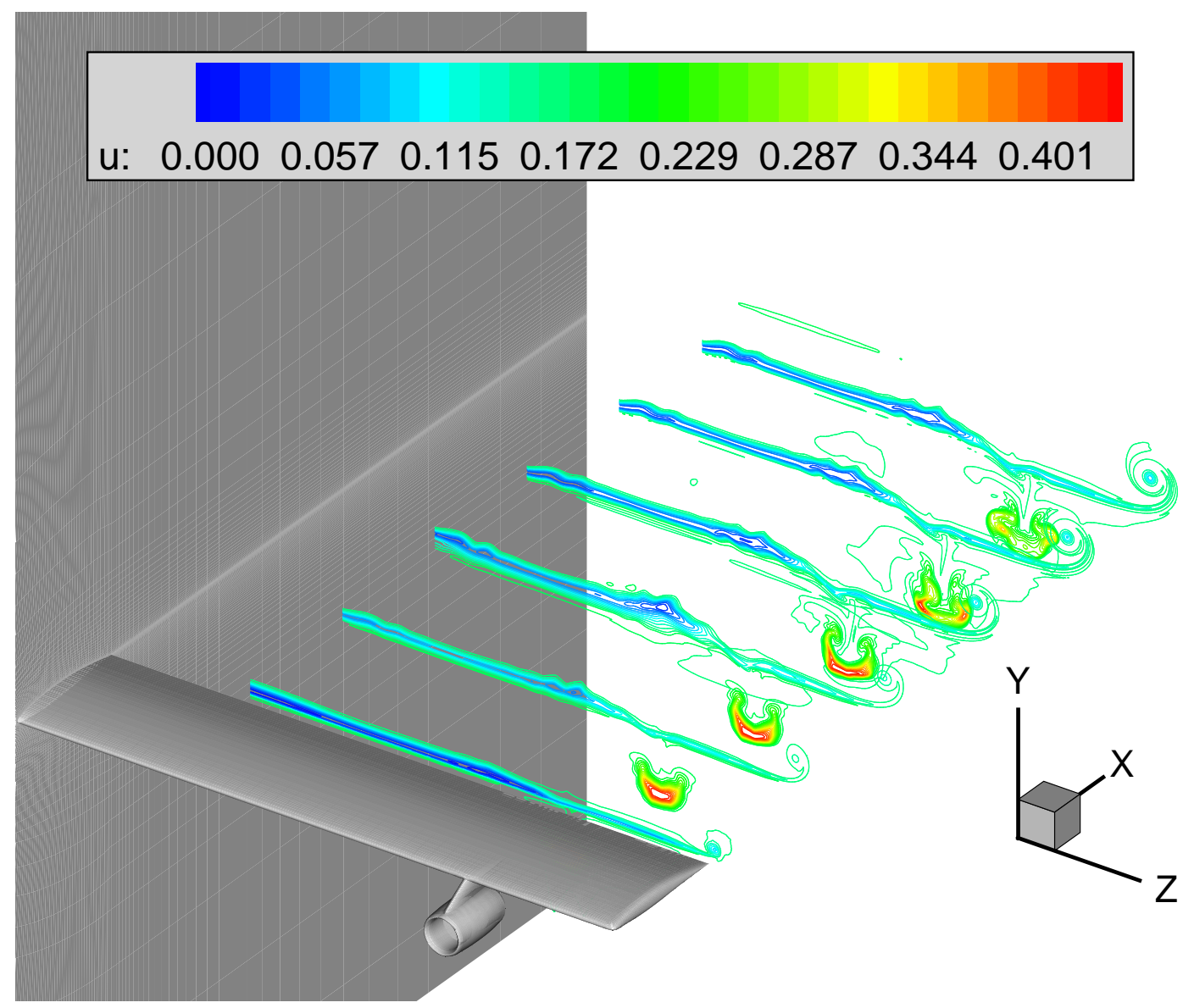

Figure 5.44: Contours of the streamwise velocity $u$ in the wake at $x / c=$ const. for pos $A$ - $t$, $u_{\text {engine }} / u_{\infty}=2.87$

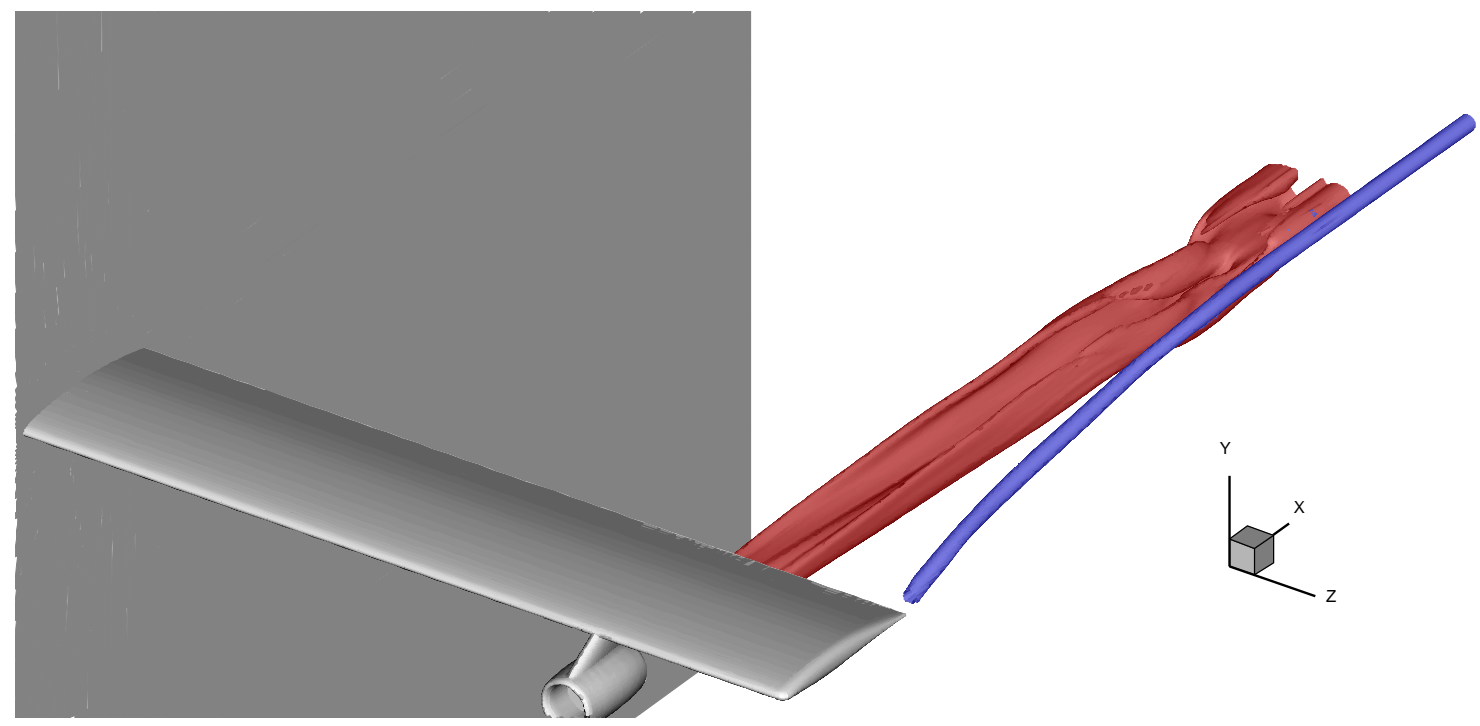

Figure 5.45: Isosurfaces of vorticity and streamwise velocity $u$ for $p o s A-t 2, u_{\text {engine }} / u_{\infty}=2.87$ 


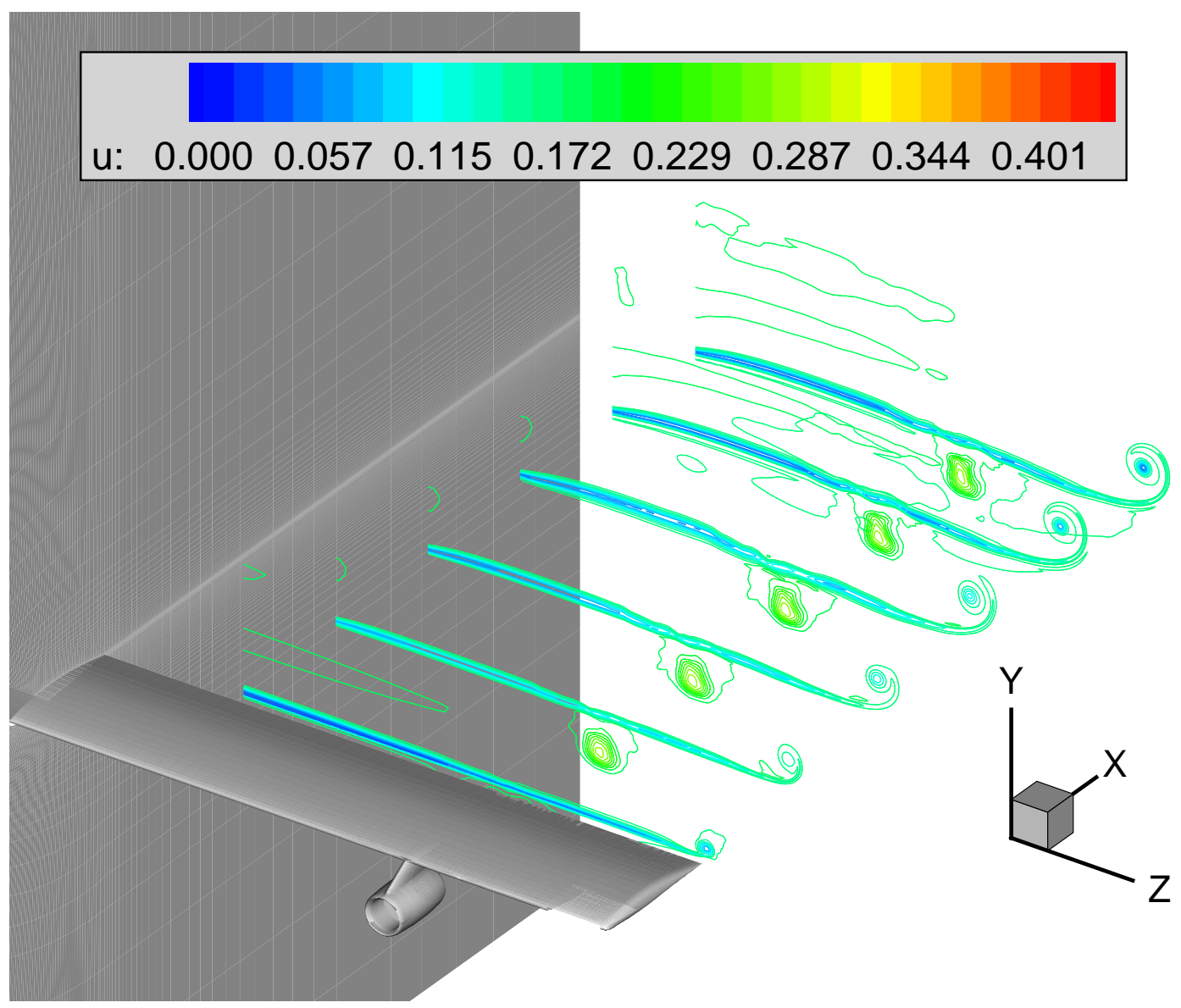

Figure 5.46: Contours of the streamwise velocity $u$ in the wake at $x / c=$ const. for pos $B$, $u_{\text {engine }} / u_{\infty}=1.77$

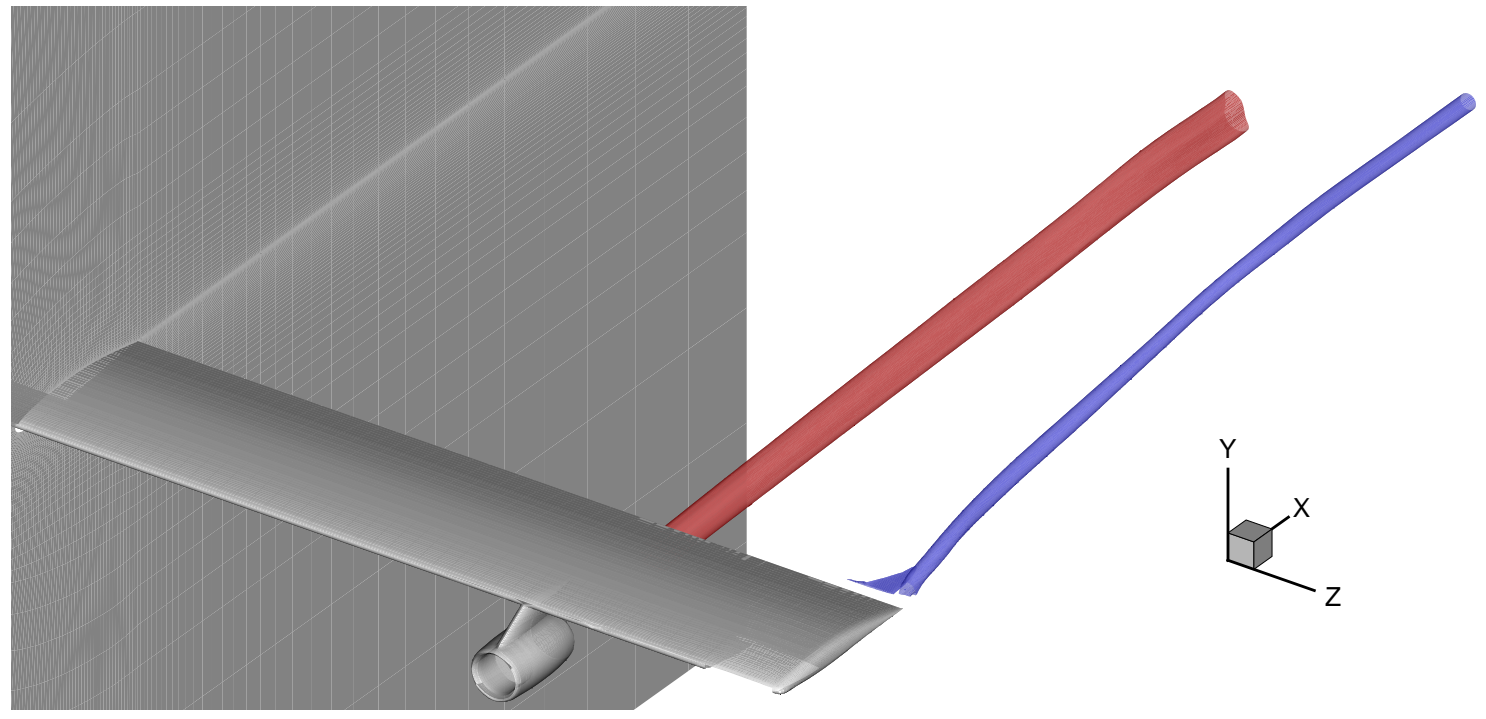

Figure 5.47: Isosurfaces of vorticity and streamwise velocity $u$ for $p o s B, u_{\text {engine }} / u_{\infty}=1.77$ 


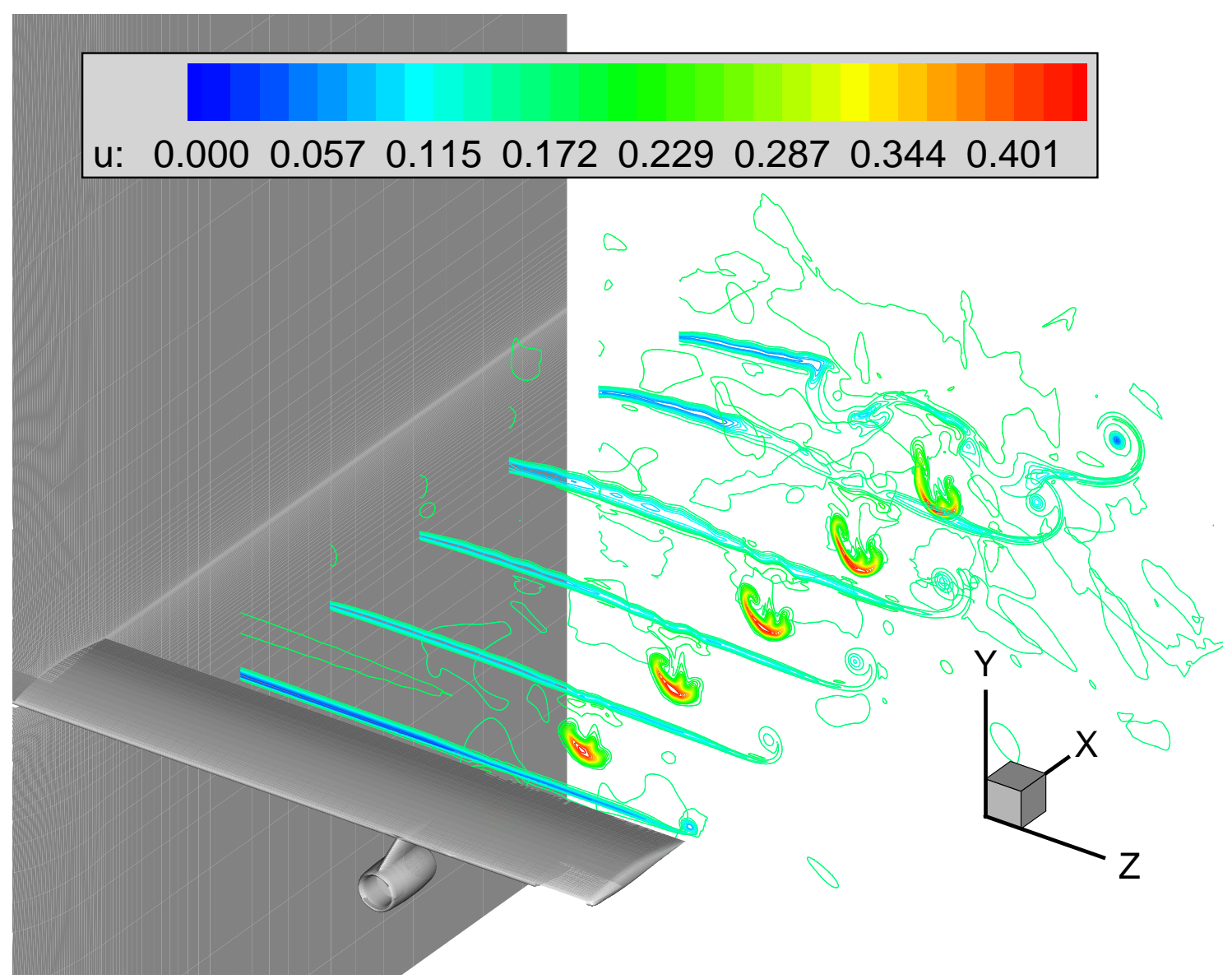

Figure 5.48: Contours of the streamwise velocity $u$ in the wake at $x / c=$ const. for posB-t2, $u_{\text {engine }} / u_{\infty}=2.87$

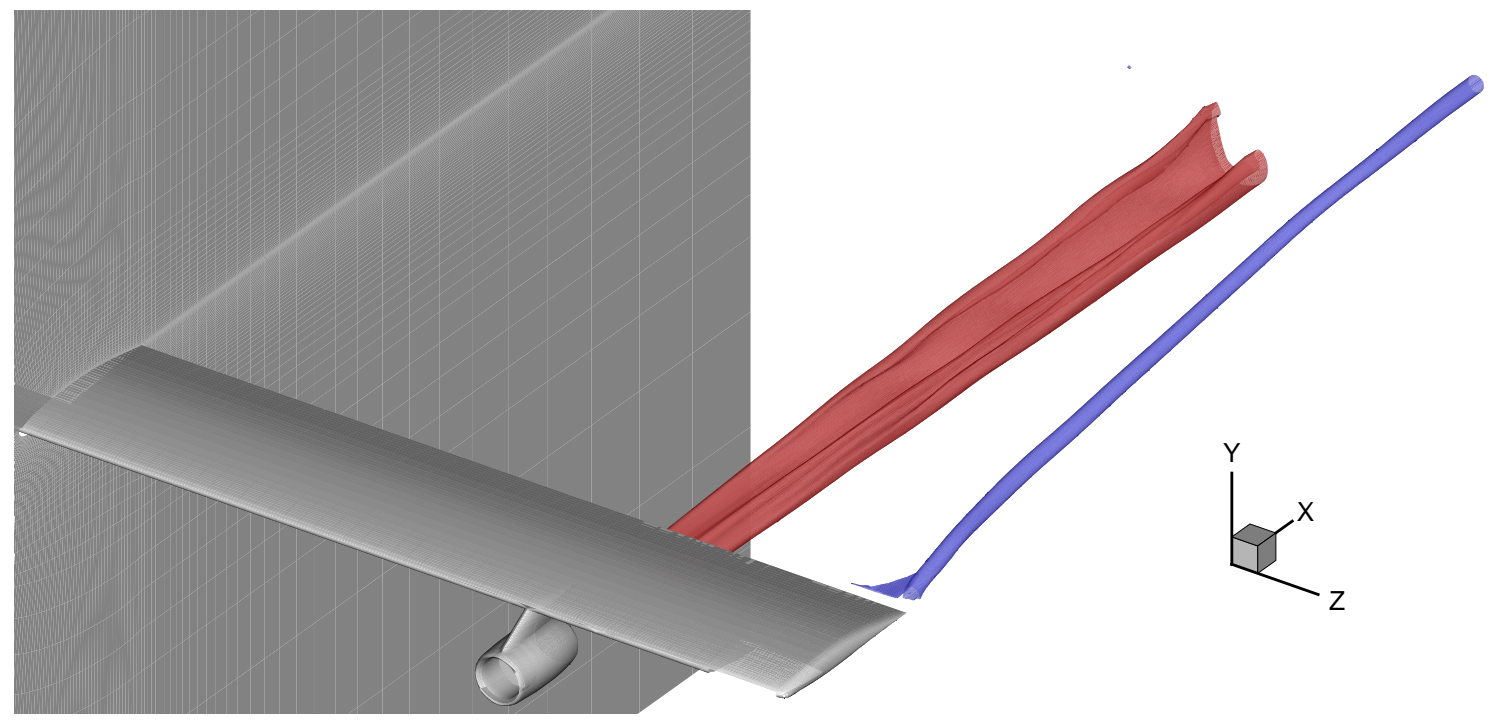

Figure 5.49: Isosurfaces of vorticity and streamwise velocity $u$ for $p o s B-t 2, u_{\text {engine }} / u_{\infty}=2.87$ 


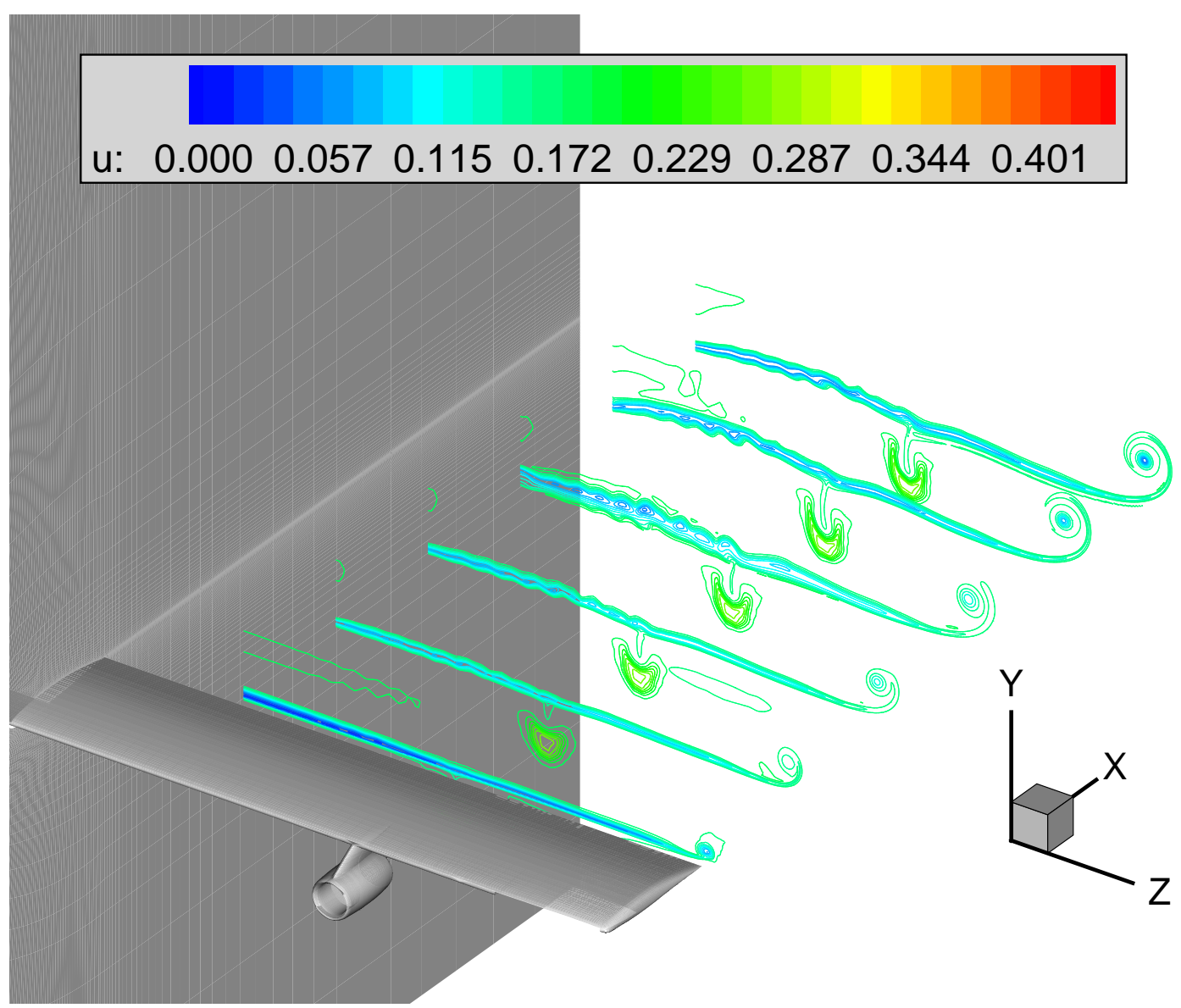

Figure 5.50: Contours of the streamwise velocity $u$ in the wake at $x / c=$ const. for $\operatorname{pos} C$, $u_{\text {engine }} / u_{\infty}=1.77$

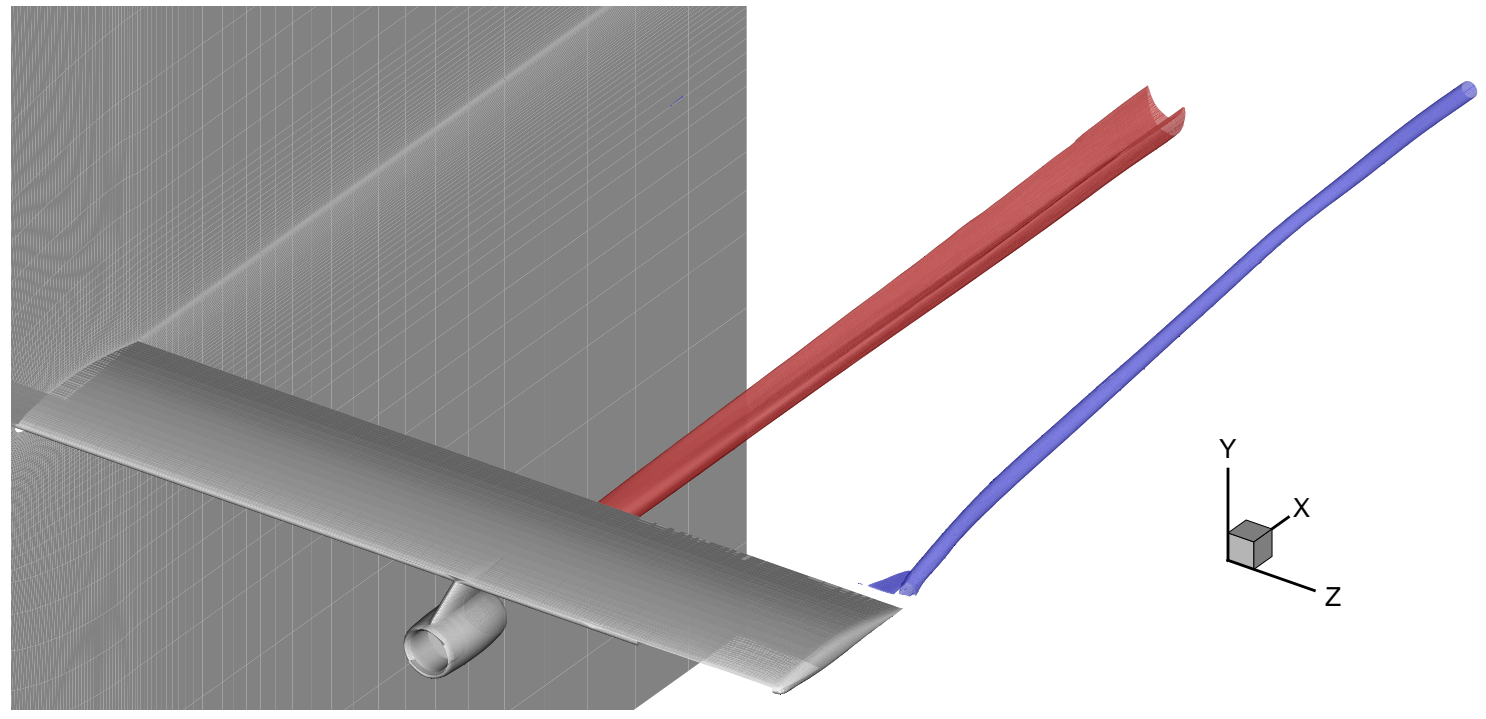

Figure 5.51: Isosurfaces of vorticity and streamwise velocity $u$ for $p o s C, u_{\text {engine }} / u_{\infty}=1.77$ 


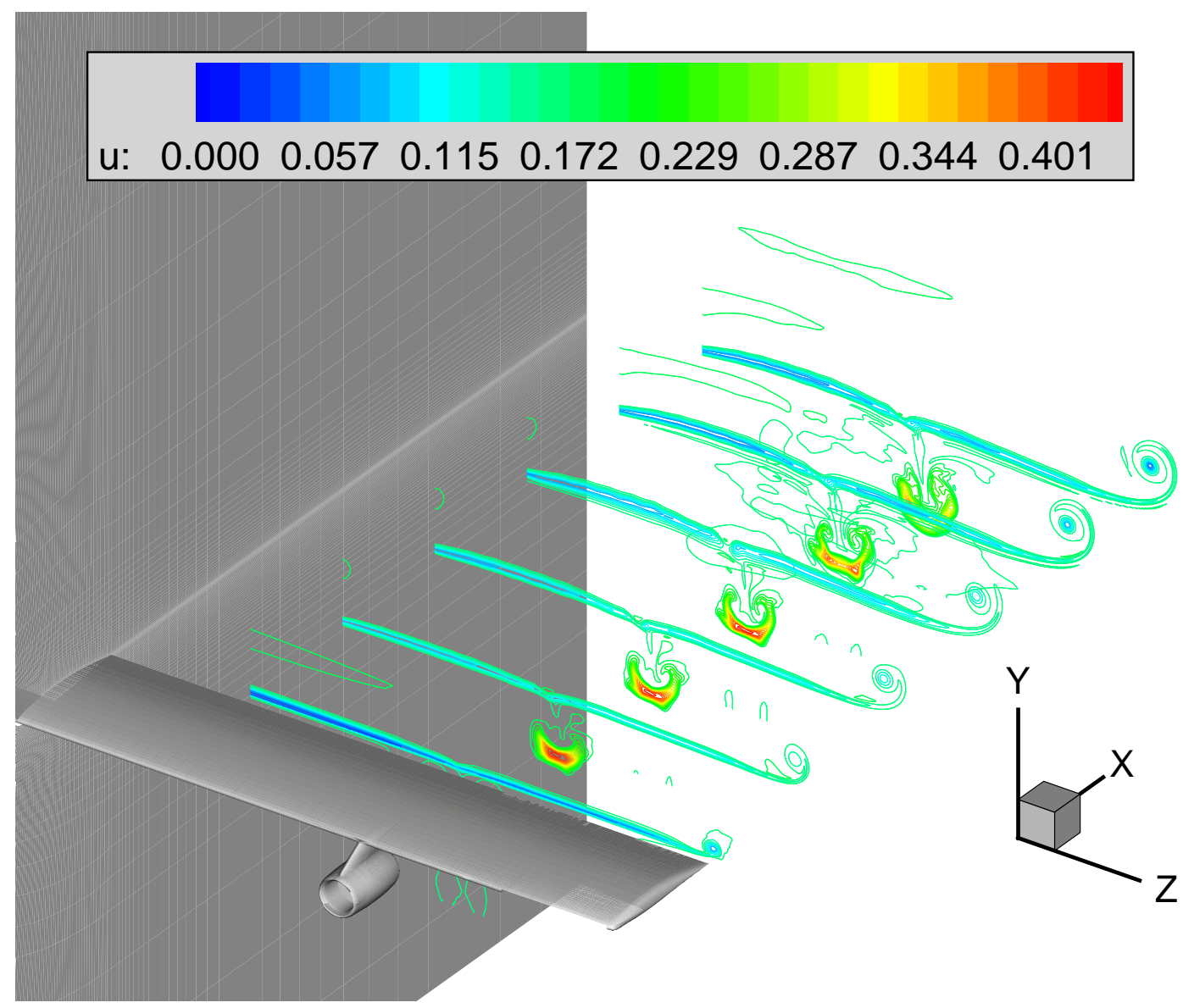

Figure 5.52: Contours of the streamwise velocity $u$ in the wake at $x / c=$ const. for $p o s C$ - $t$, $u_{\text {engine }} / u_{\infty}=2.87$

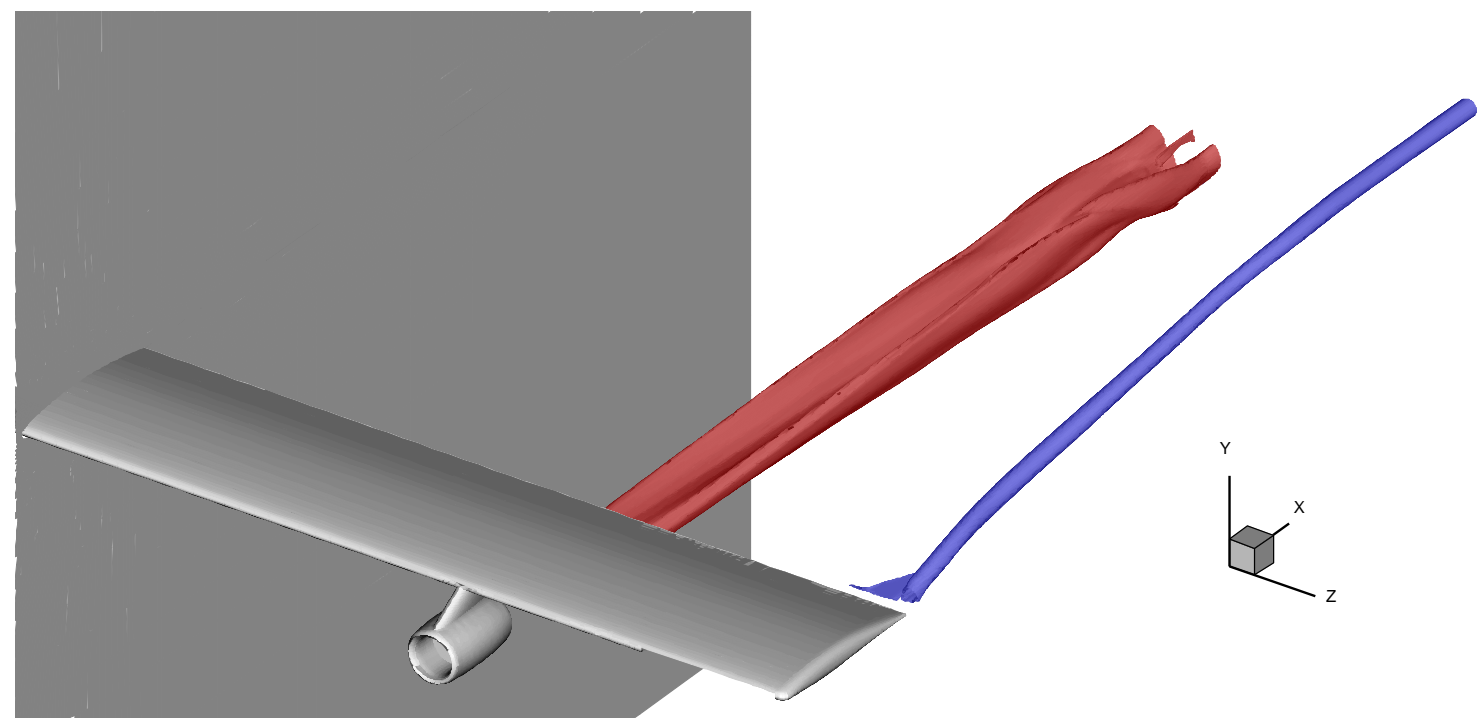

Figure 5.53: $\quad$ Isosurfaces of vorticity and streamwise velocity $u$ for $p o s C$ - $t 2, u_{\text {engine }} / u_{\infty}=2.87$ 


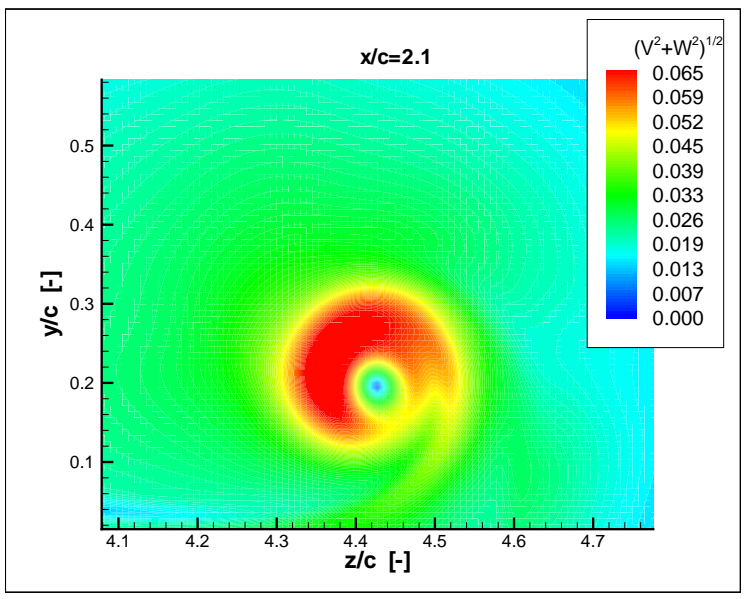

Engine position A $\quad u_{\text {engine }} / u_{\infty}=1.77$

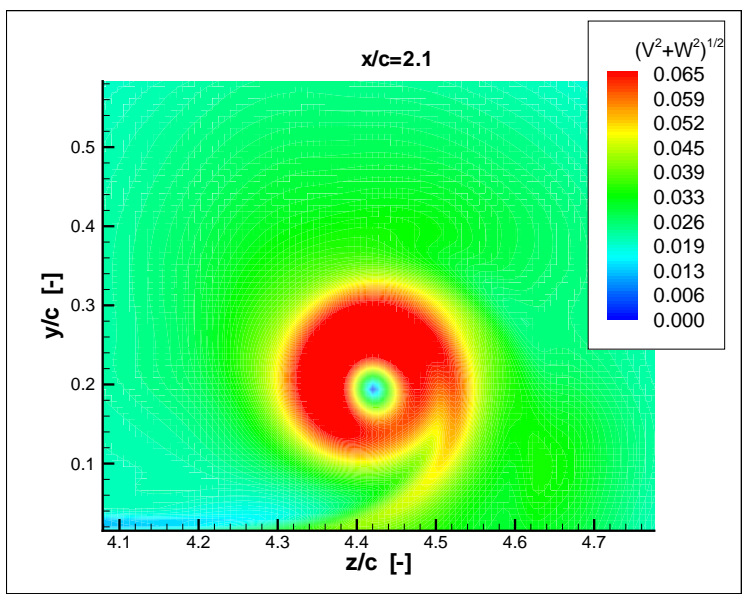

Engine position B $\quad u_{\text {engine }} / u_{\infty}=1.77$

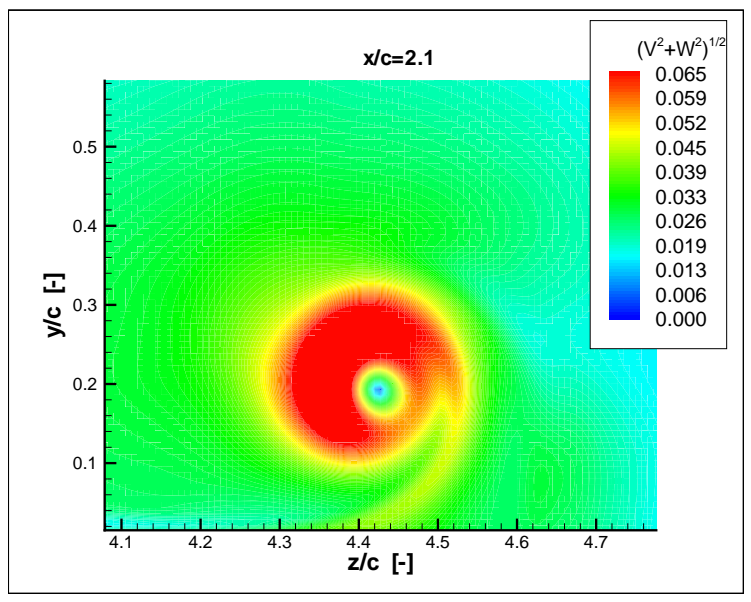

Engine position $\mathrm{C} \quad u_{\text {engine }} / u_{\infty}=1.77$

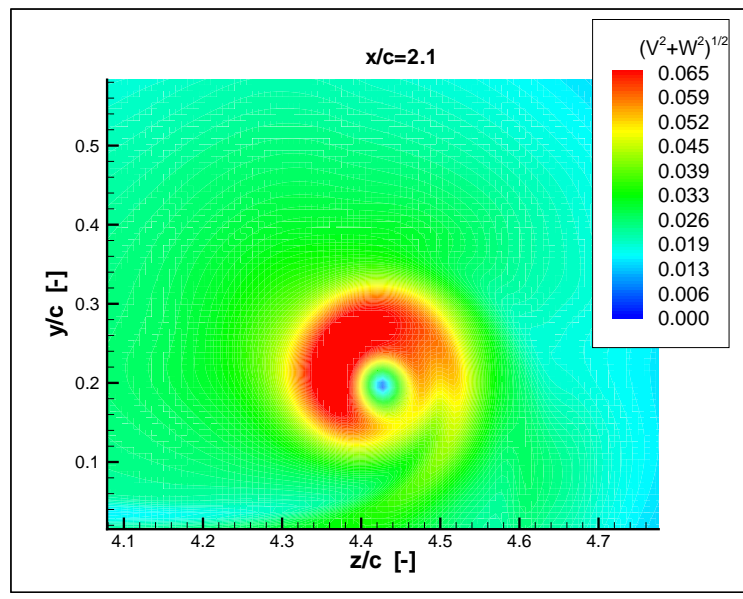

Engine position A $\quad u_{\text {engine }} / u_{\infty}=2.87$

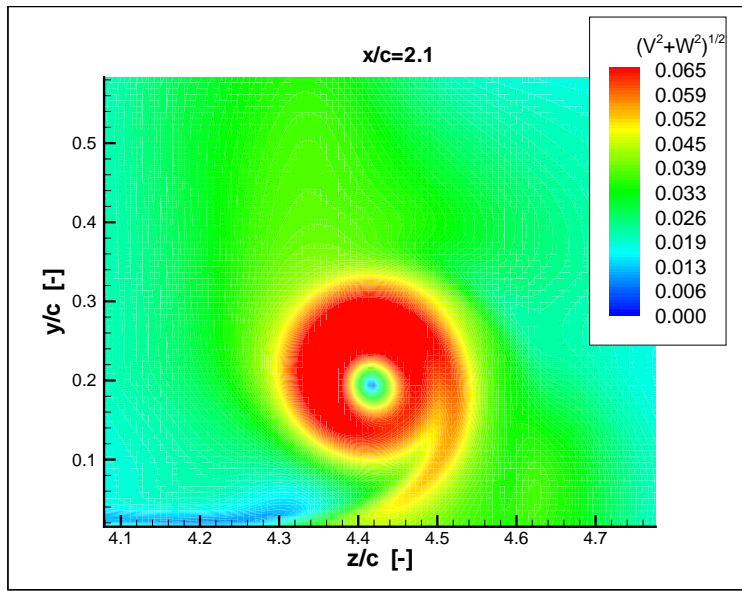

Engine position B $\quad u_{\text {engine }} / u_{\infty}=2.87$

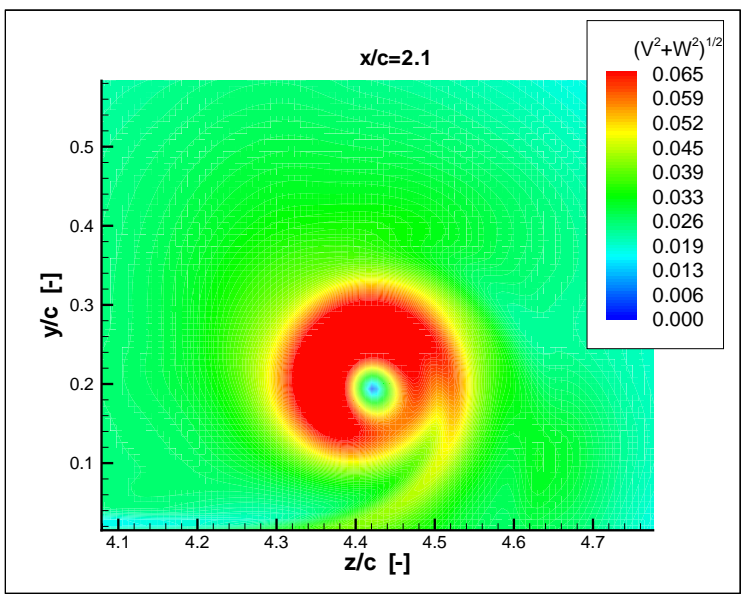

Engine position $\mathrm{C} \quad u_{\text {engine }} / u_{\infty}=2.87$

Figure 5.54: Velocity contours $\sqrt{v^{2}+w^{2}}$ at $x / c=2.1$ for $M a_{\infty}=0.18, R e_{c}=268250$ and $\alpha=8.0^{\circ}$ 


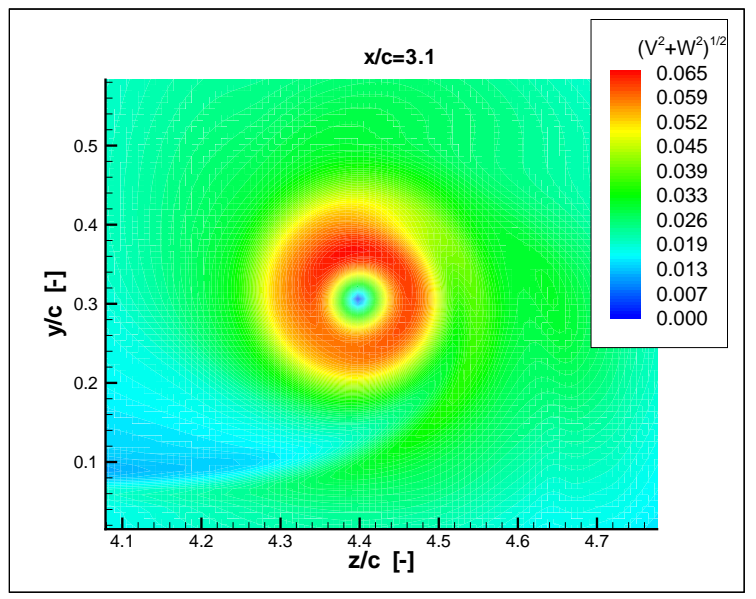

Engine position A $\quad u_{\text {engine }} / u_{\infty}=1.77$

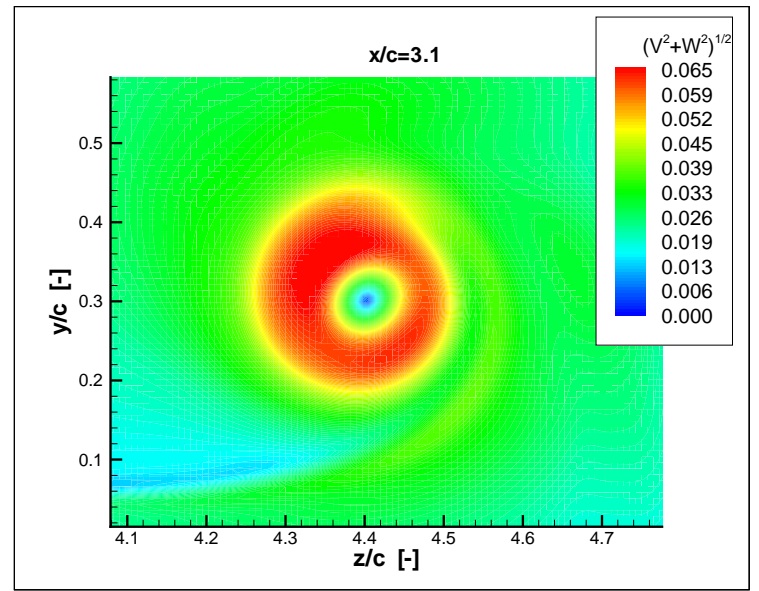

Engine position B $\quad u_{\text {engine }} / u_{\infty}=1.77$

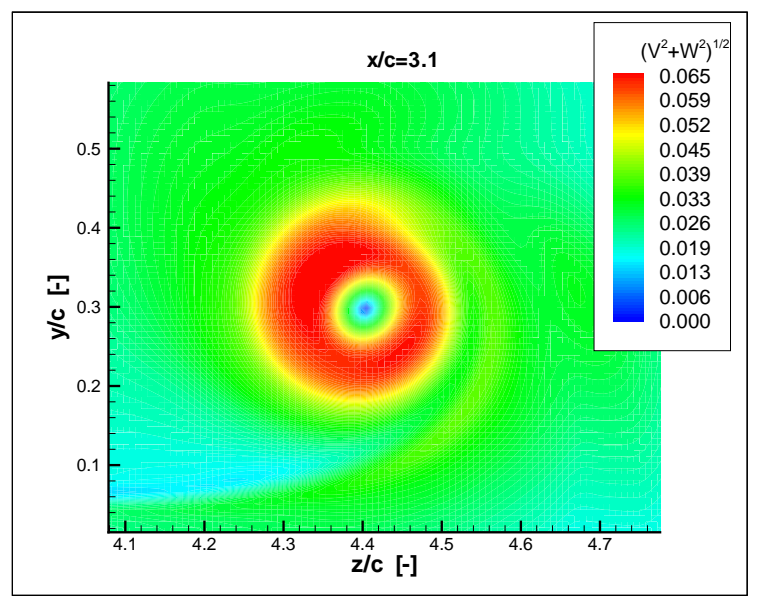

Engine position $\mathrm{C} \quad u_{\text {engine }} / u_{\infty}=1.77$

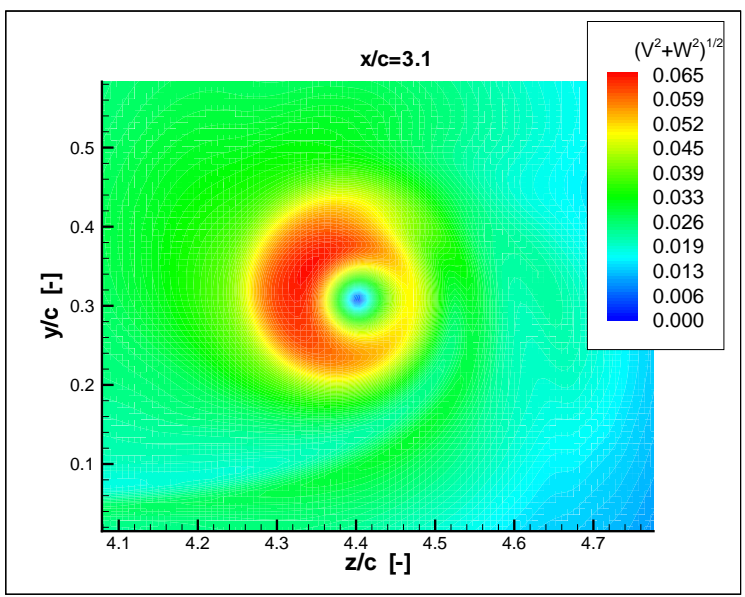

Engine position A $u_{\text {engine }} / u_{\infty}=2.87$

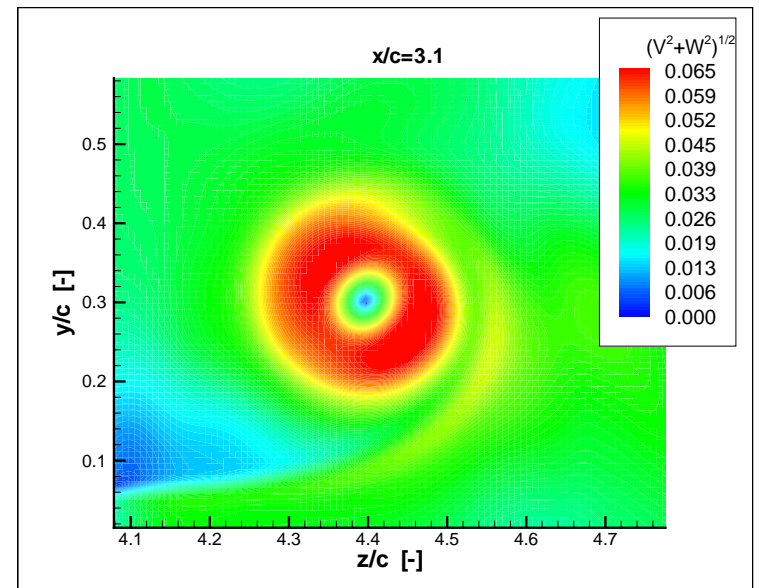

Engine position B $\quad u_{\text {engine }} / u_{\infty}=2.87$

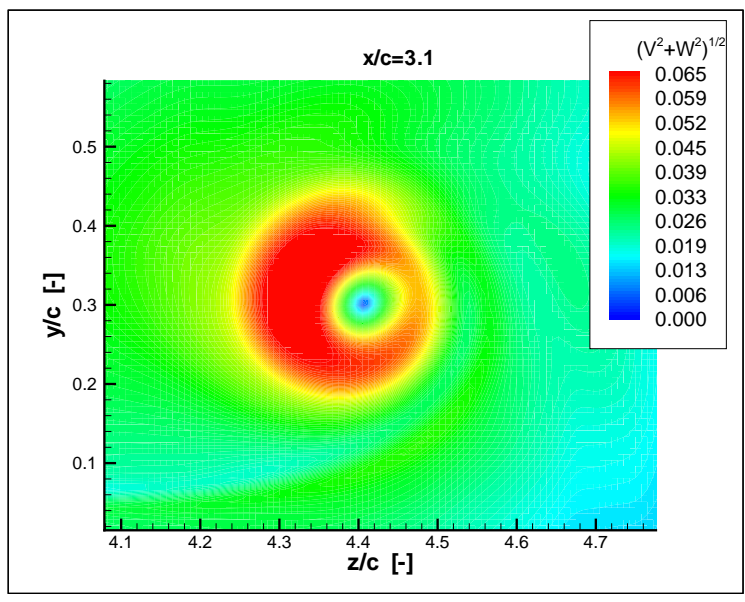

Engine position $\mathrm{C} \quad u_{\text {engine }} / u_{\infty}=2.87$

Figure 5.55: Velocity contours $\sqrt{v^{2}+w^{2}}$ at $x / c=3.1$ for $M a_{\infty}=0.18, R e_{c}=268250$ and $\alpha=8.0^{\circ}$ 


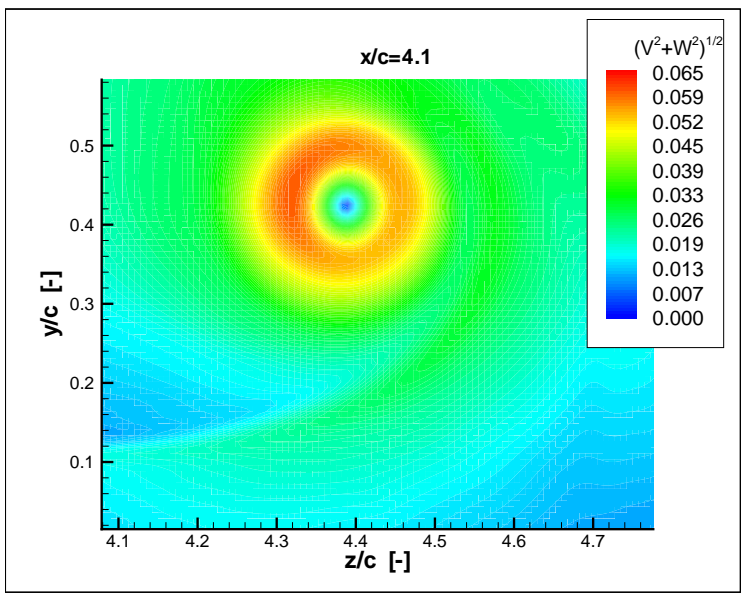

Engine position A $\quad u_{\text {engine }} / u_{\infty}=1.77$

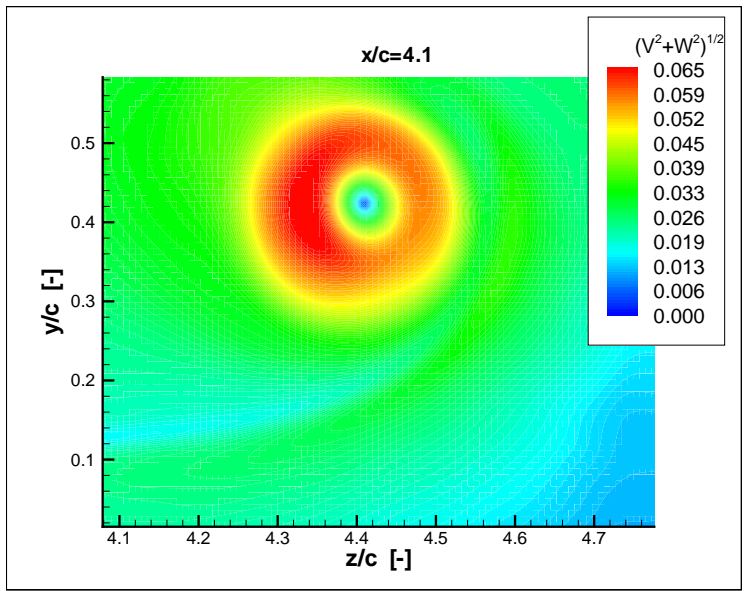

Engine position B $\quad u_{\text {engine }} / u_{\infty}=1.77$

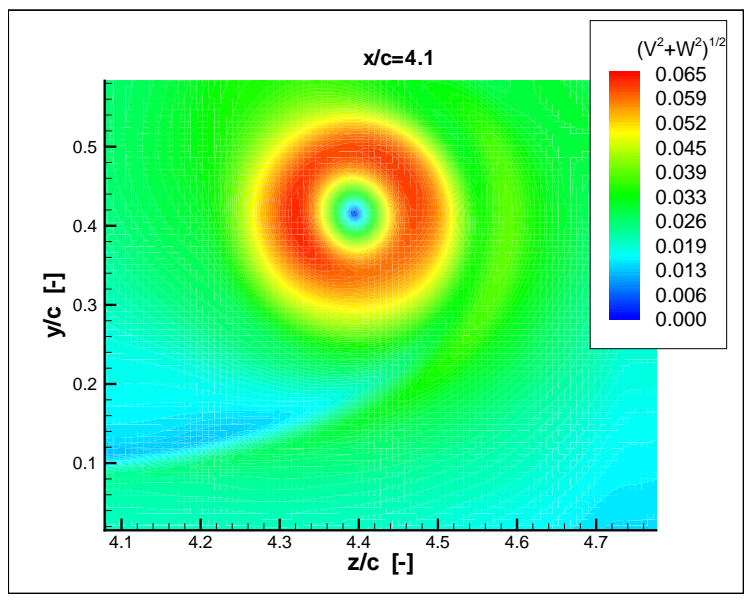

Engine position $\mathrm{C} \quad u_{\text {engine }} / u_{\infty}=1.77$

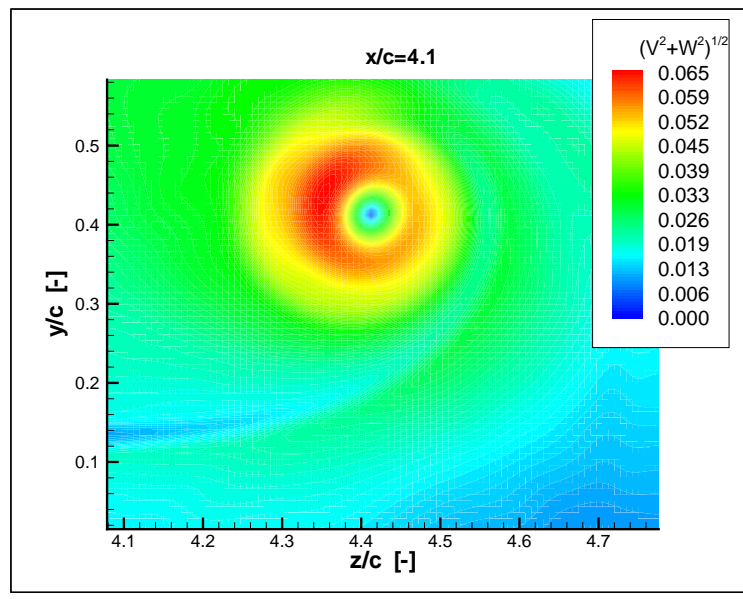

Engine position A $\quad u_{\text {engine }} / u_{\infty}=2.87$

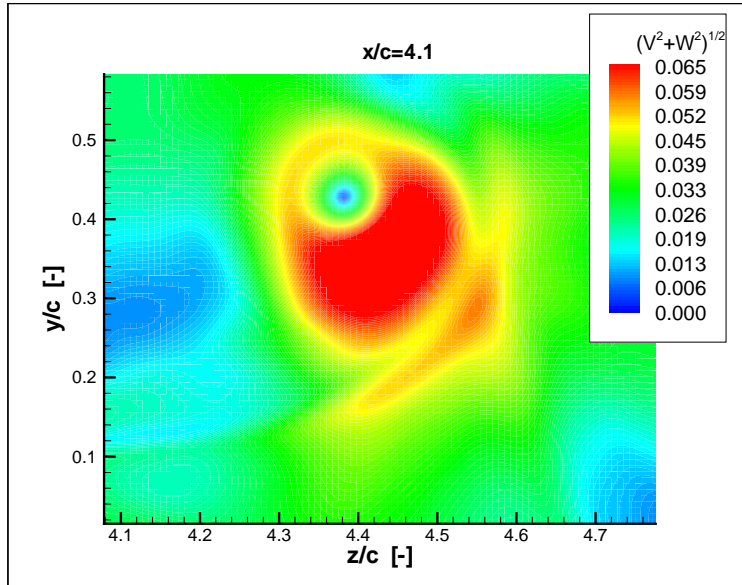

Engine position B $\quad u_{\text {engine }} / u_{\infty}=2.87$

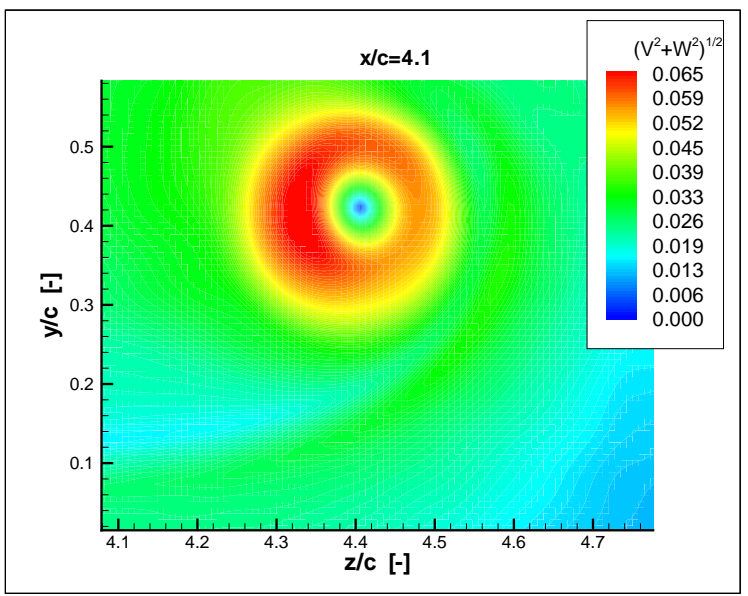

Engine position $\mathrm{C} \quad u_{\text {engine }} / u_{\infty}=2.87$

Figure 5.56: Velocity contours $\sqrt{v^{2}+w^{2}}$ at $x / c=4.1$ for $M a_{\infty}=0.18, R e_{c}=268250$ and $\alpha=8.0^{\circ}$ 


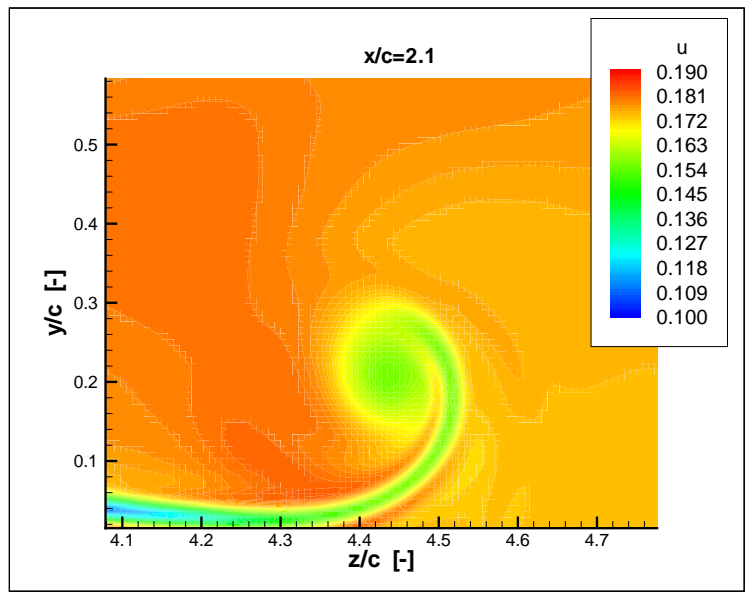

Engine position A $\quad u_{\text {engine }} / u_{\infty}=1.77$

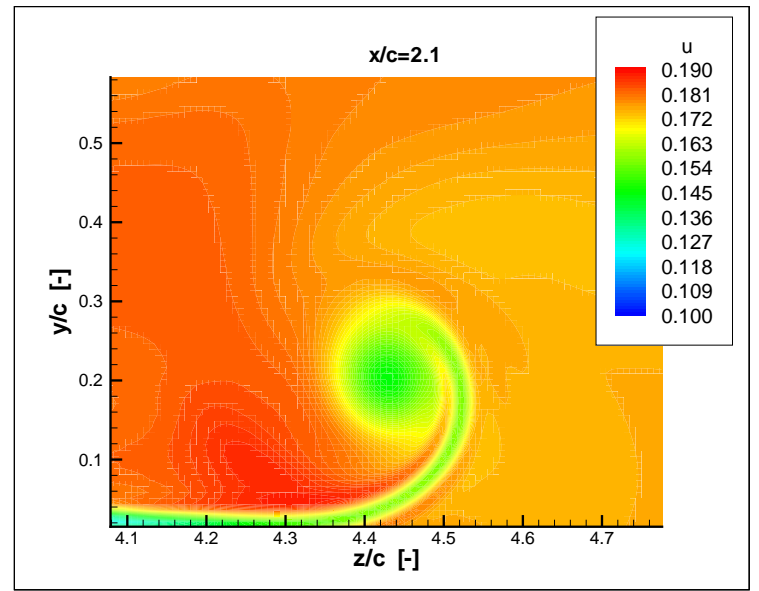

Engine position B $\quad u_{\text {engine }} / u_{\infty}=1.77$

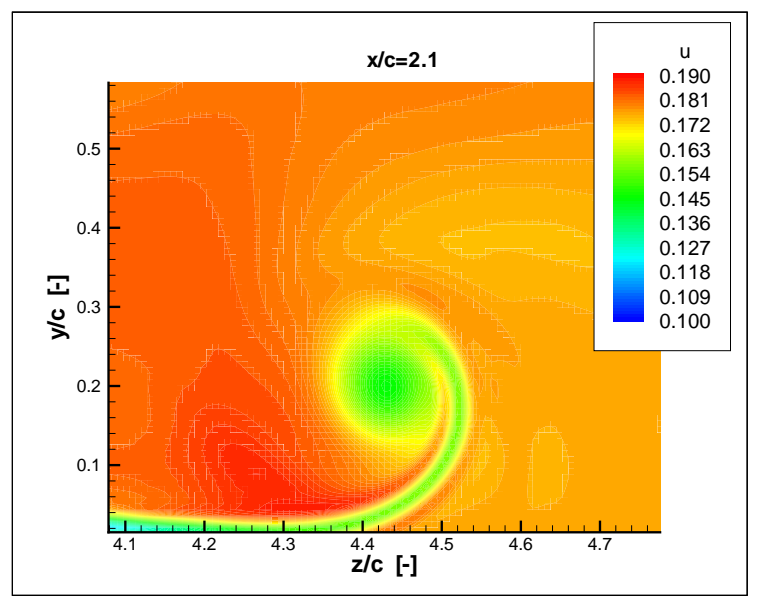

Engine position $\mathrm{C} \quad u_{\text {engine }} / u_{\infty}=1.77$

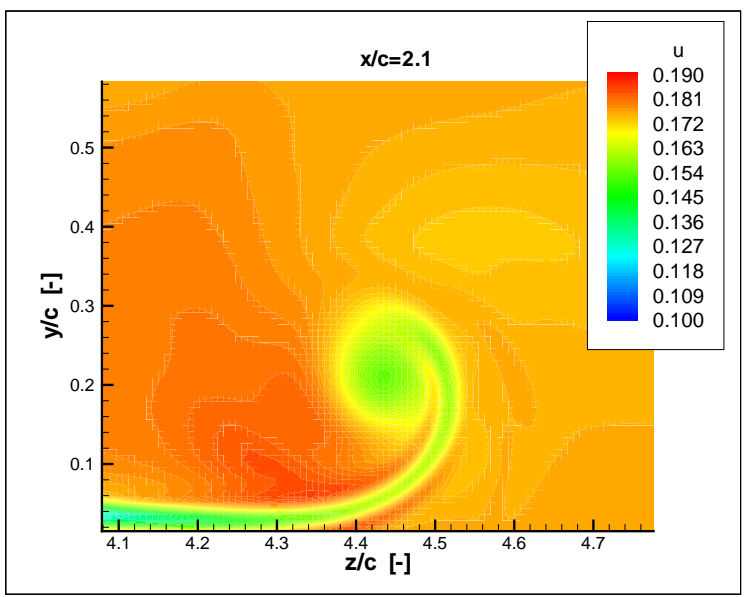

Engine position A $u_{\text {engine }} / u_{\infty}=2.87$

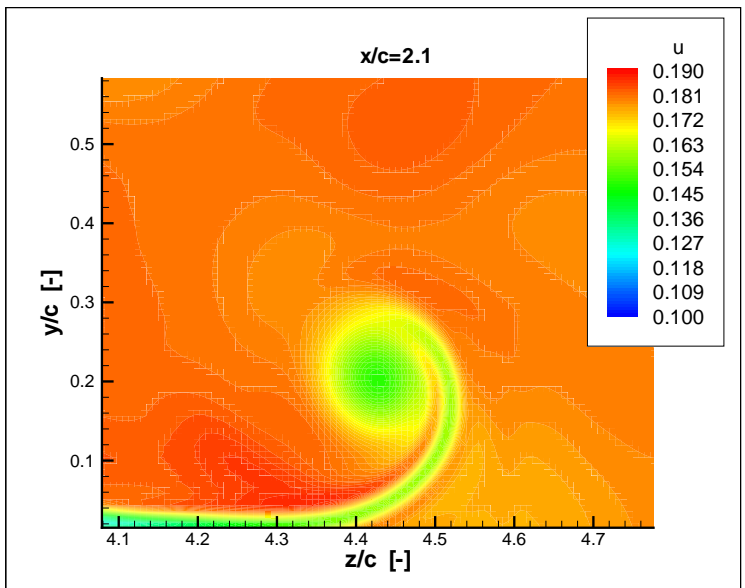

Engine position B $\quad u_{\text {engine }} / u_{\infty}=2.87$

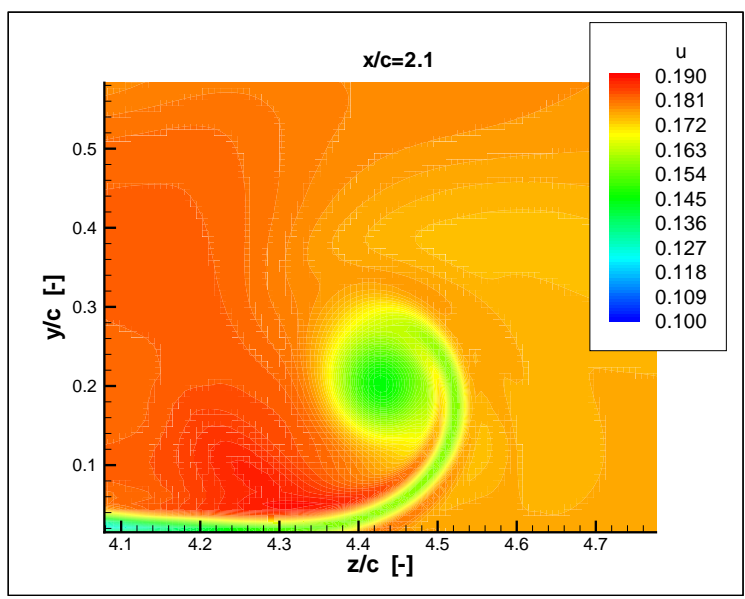

Engine position $\mathrm{C} \quad u_{\text {engine }} / u_{\infty}=2.87$

Figure 5.57: Contours of streamwise velocity $u$ at $x / c=2.1$ for $M a_{\infty}=0.18, R e_{c}=268250$ and $\alpha=8.0^{\circ}$ 


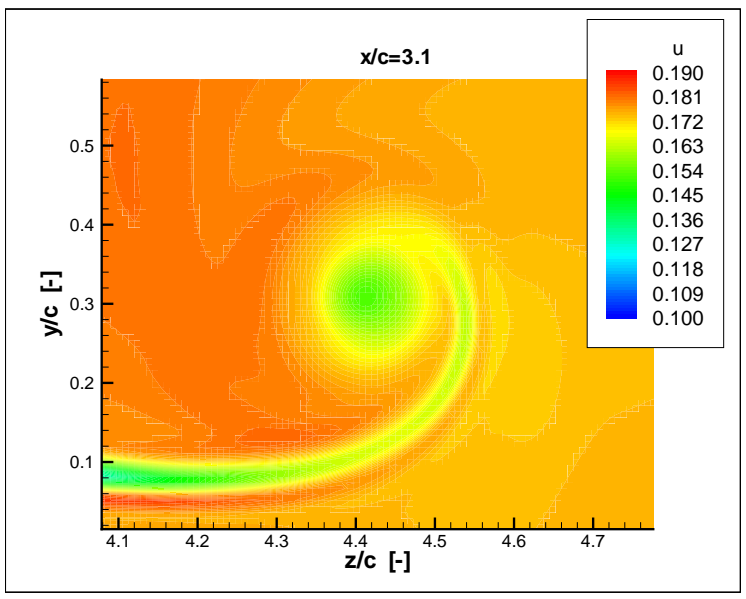

Engine position A $\quad u_{\text {engine }} / u_{\infty}=1.77$

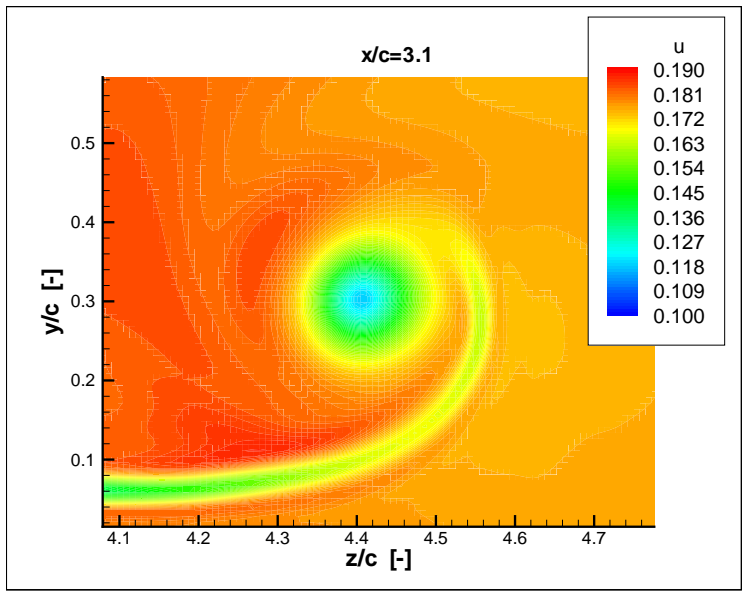

Engine position B $\quad u_{\text {engine }} / u_{\infty}=1.77$

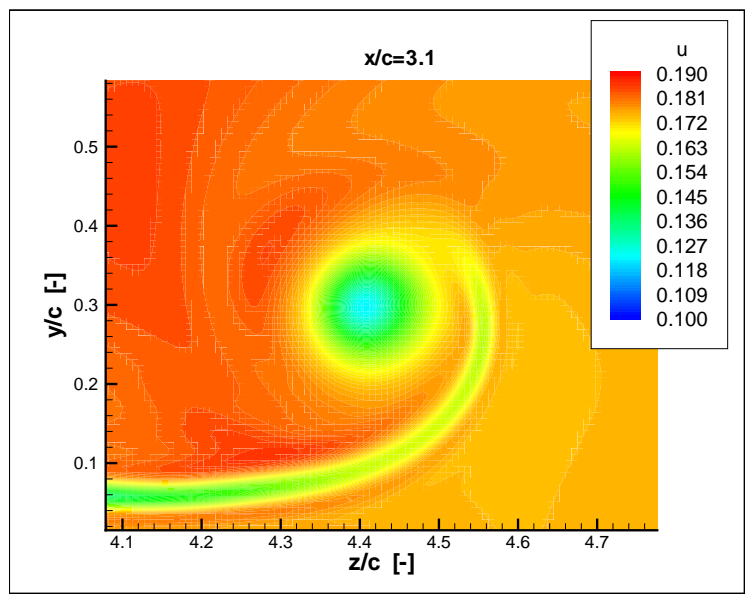

Engine position $\mathrm{C} \quad u_{\text {engine }} / u_{\infty}=1.77$

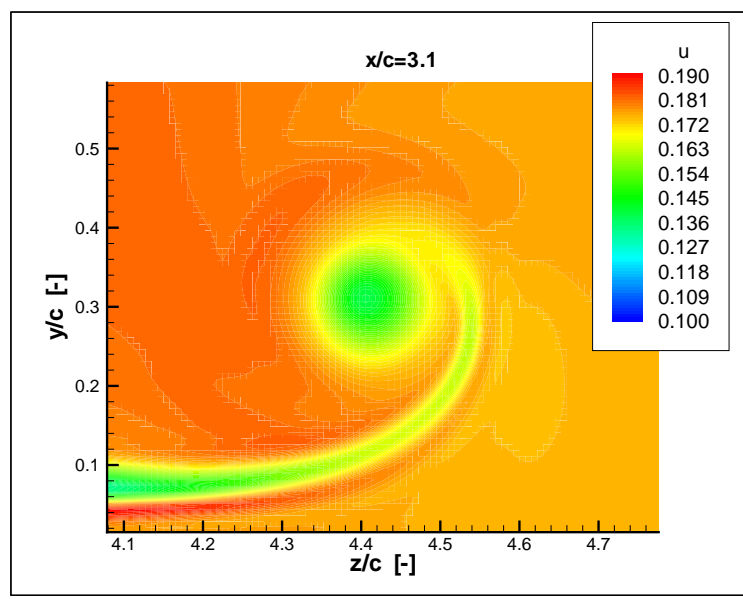

Engine position A $\quad u_{\text {engine }} / u_{\infty}=2.87$

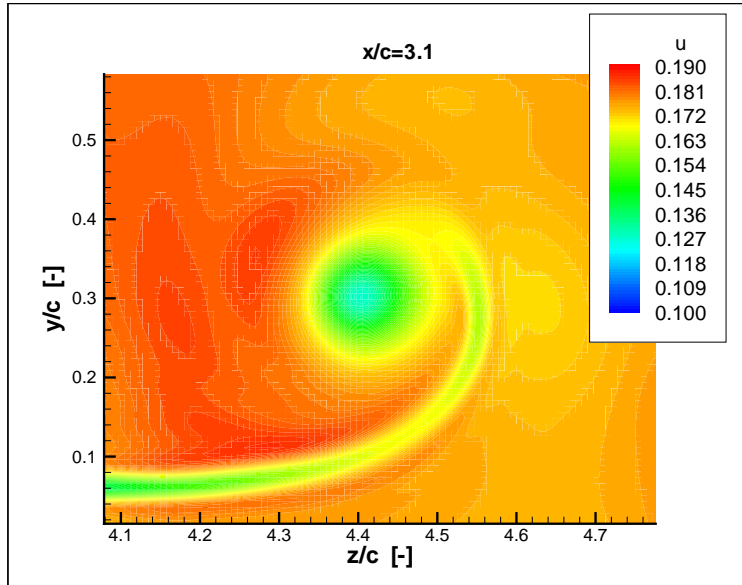

Engine position B $\quad u_{\text {engine }} / u_{\infty}=2.87$

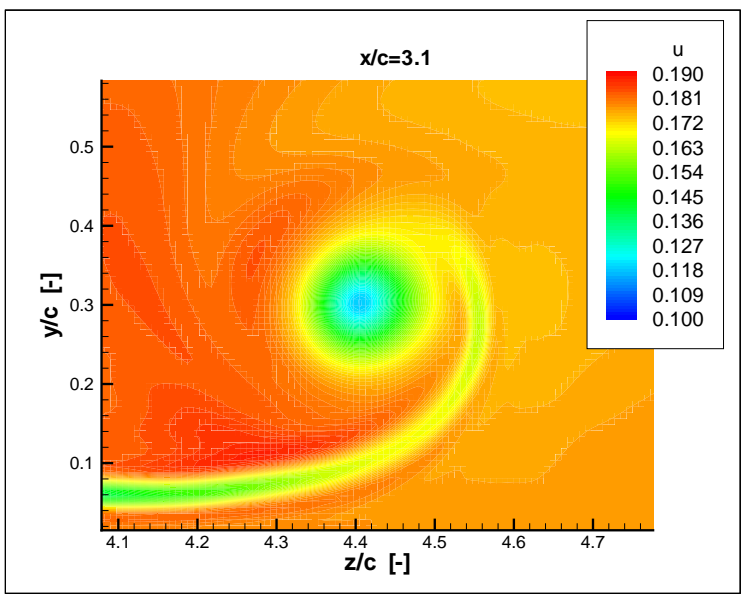

Engine position $\mathrm{C} \quad u_{\text {engine }} / u_{\infty}=2.87$

Figure 5.58: Contours of streamwise velocity $u$ at $x / c=3.1$ for $M a_{\infty}=0.18, R e_{c}=268250$ and $\alpha=8.0^{\circ}$ 


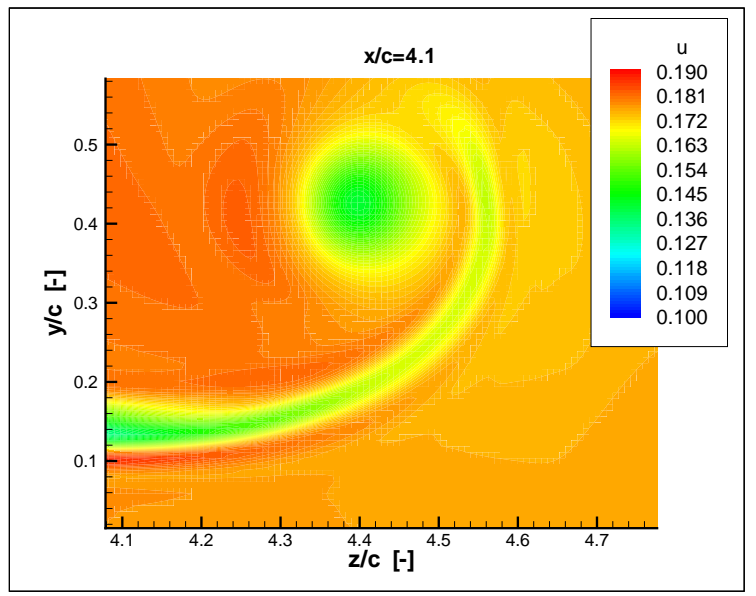

Engine position A $\quad u_{\text {engine }} / u_{\infty}=1.77$

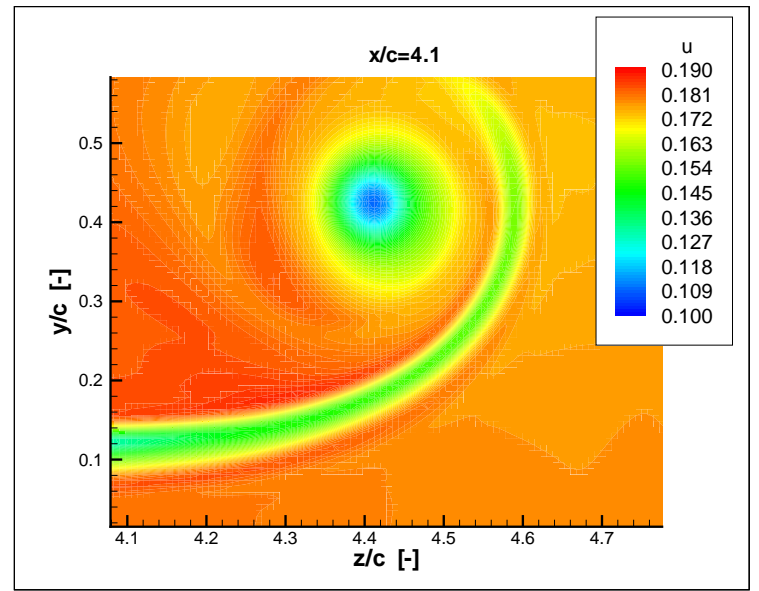

Engine position B $\quad u_{\text {engine }} / u_{\infty}=1.77$

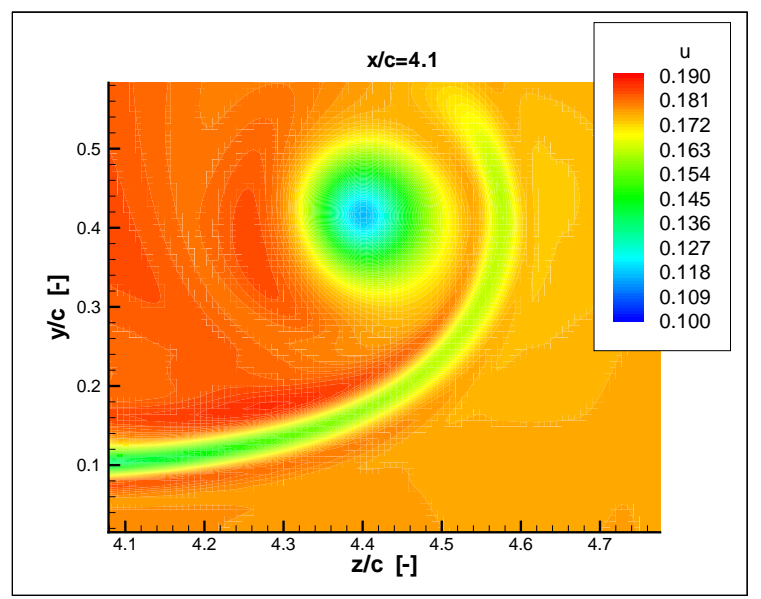

Engine position $\mathrm{C} \quad u_{\text {engine }} / u_{\infty}=1.77$

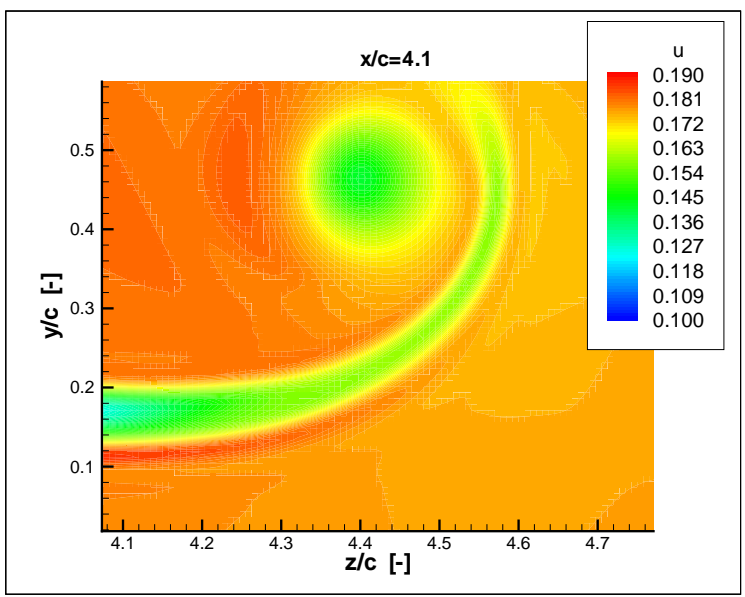

Engine position A $u_{\text {engine }} / u_{\infty}=2.87$

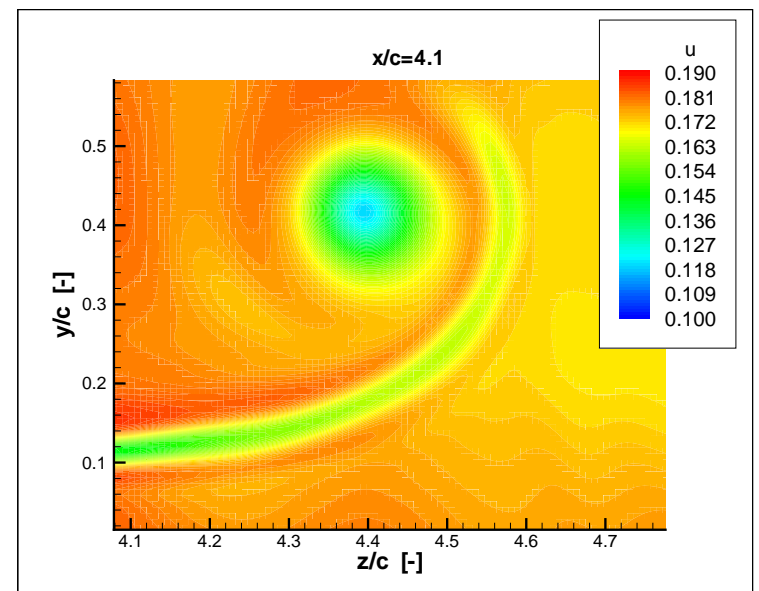

Engine position B $\quad u_{\text {engine }} / u_{\infty}=2.87$

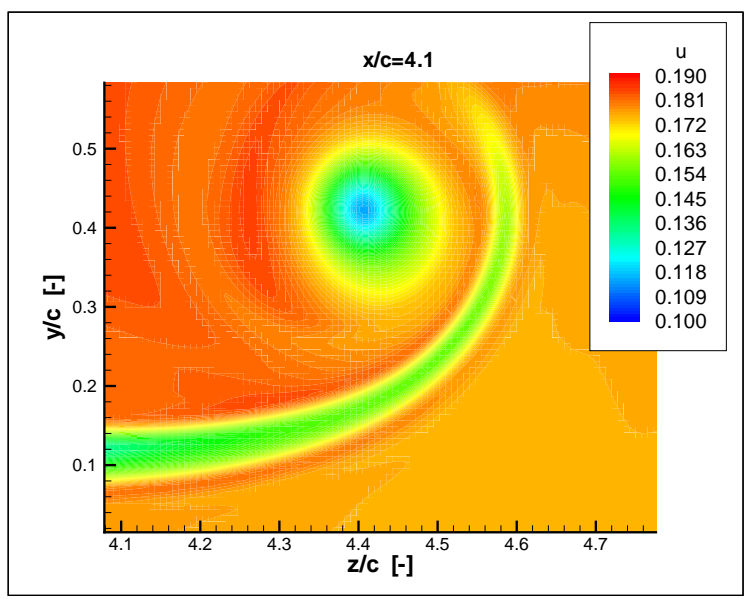

Engine position $\mathrm{C} \quad u_{\text {engine }} / u_{\infty}=2.87$

Figure 5.59: Contours of streamwise velocity $u$ at $x / c=4.1$ for $M a_{\infty}=0.18, R e_{c}=268250$ and $\alpha=8.0^{\circ}$ 

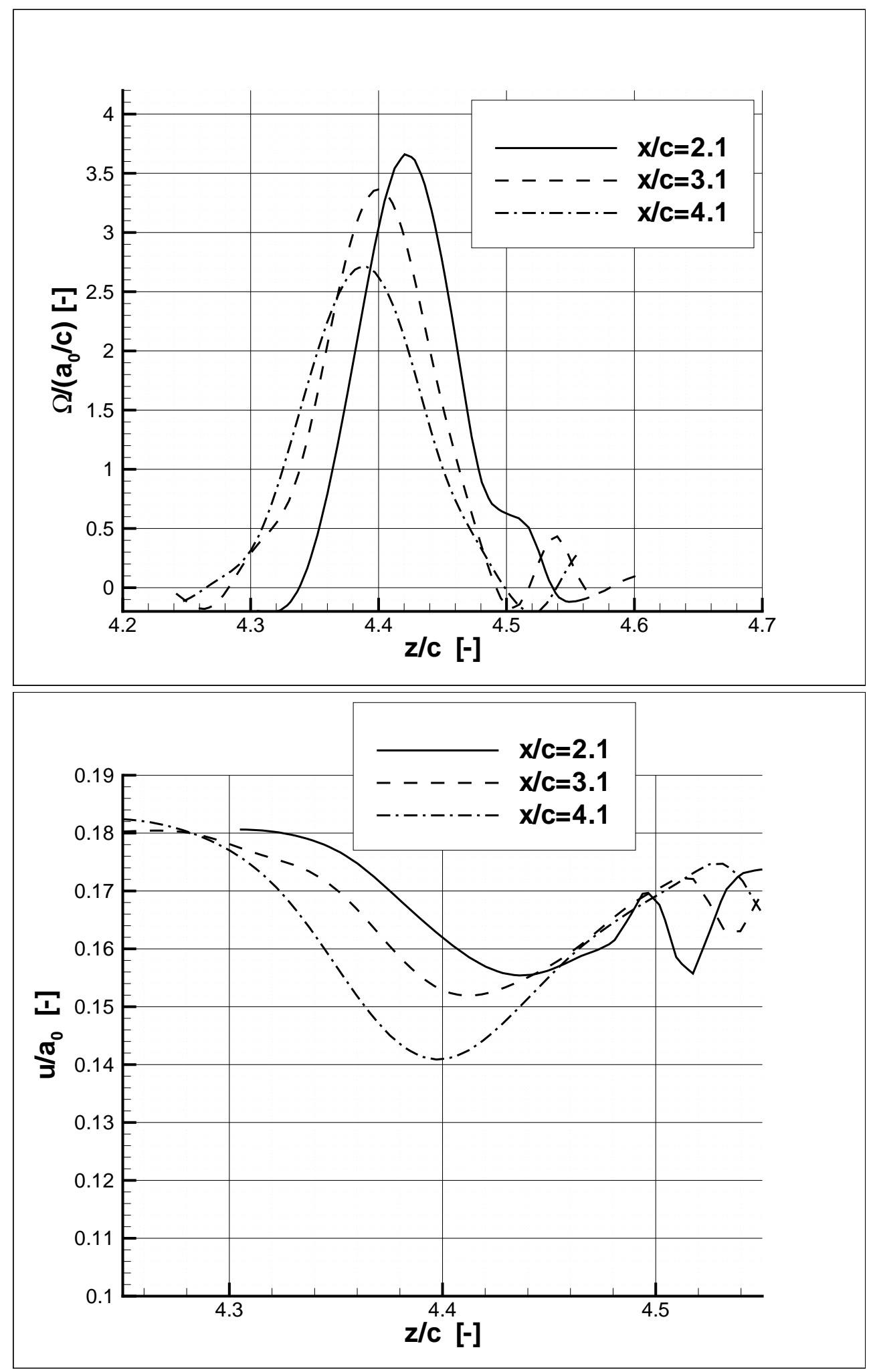

Figure 5.60: Vorticity $\Omega$ (top) and streamwise velocity $u$ (bottom) profiles vs. $z / c$ for pos $A, u_{\text {engine }} / u_{\infty}=1.77$ 

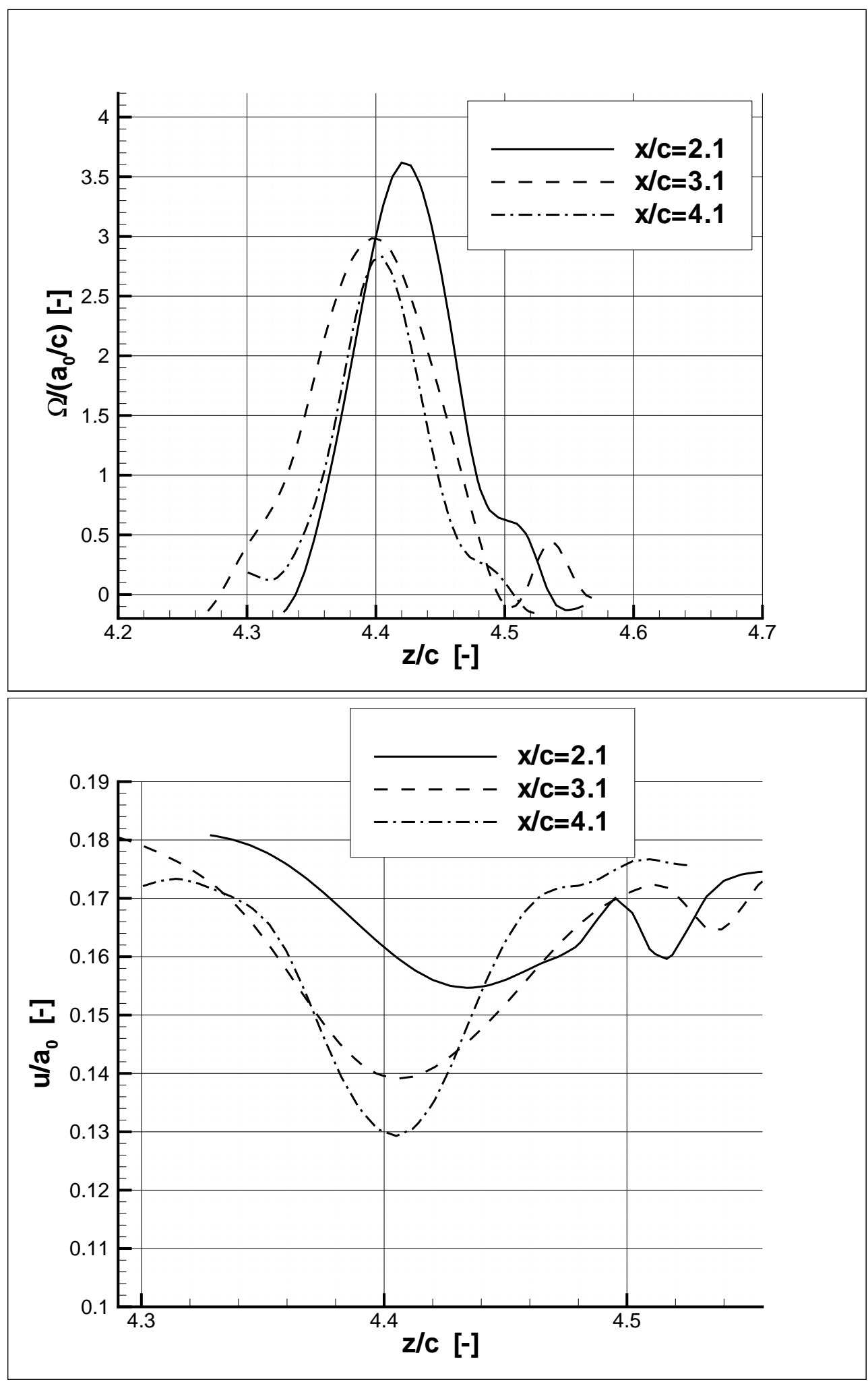

Figure 5.61: Vorticity $\Omega$ (top) and streamwise velocity $u$ (bottom) profiles vs. $z / c$ for posA-t $2, u_{\text {engine }} / u_{\infty}=2.87$ 


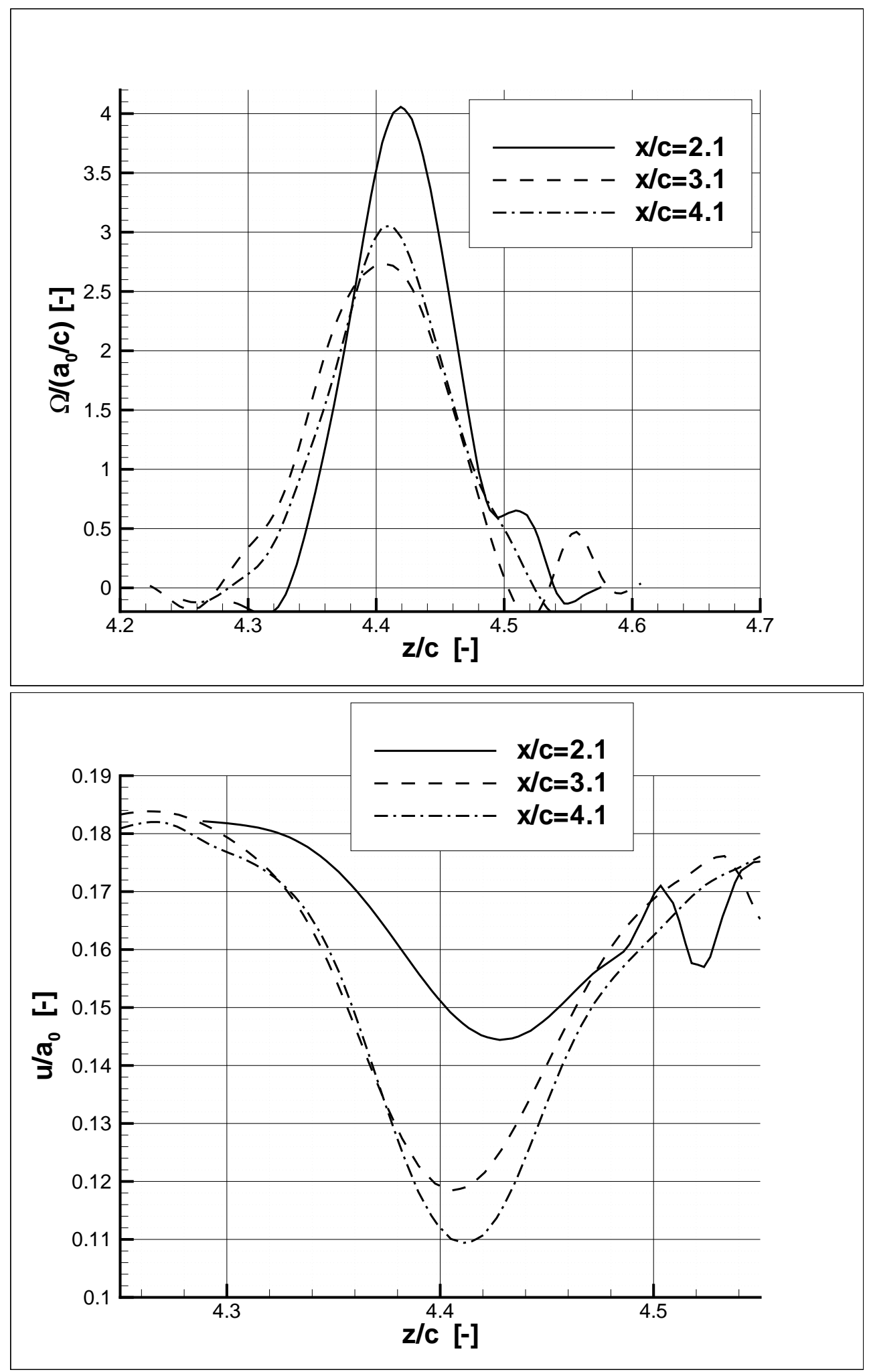

Figure 5.62: Vorticity $\Omega$ (top) and streamwise velocity $u$ (bottom) profiles vs. $z / c$ for pos $B, u_{\text {engine }} / u_{\infty}=1.77$ 

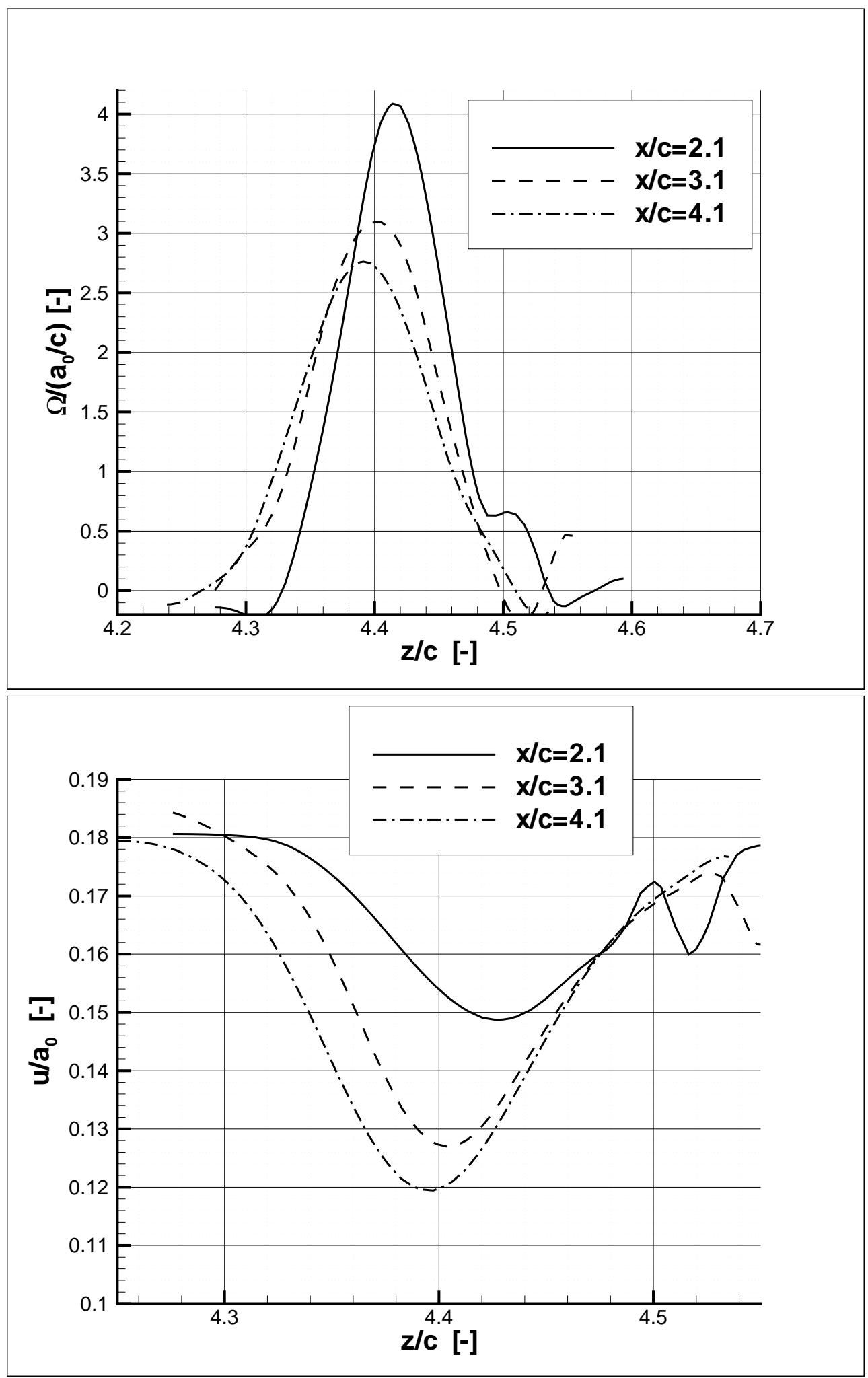

Figure 5.63: Vorticity $\Omega$ (top) and streamwise velocity $u$ (bottom) profiles vs. $z / c$ for posB-t2, $u_{\text {engine }} / u_{\infty}=2.87$ 

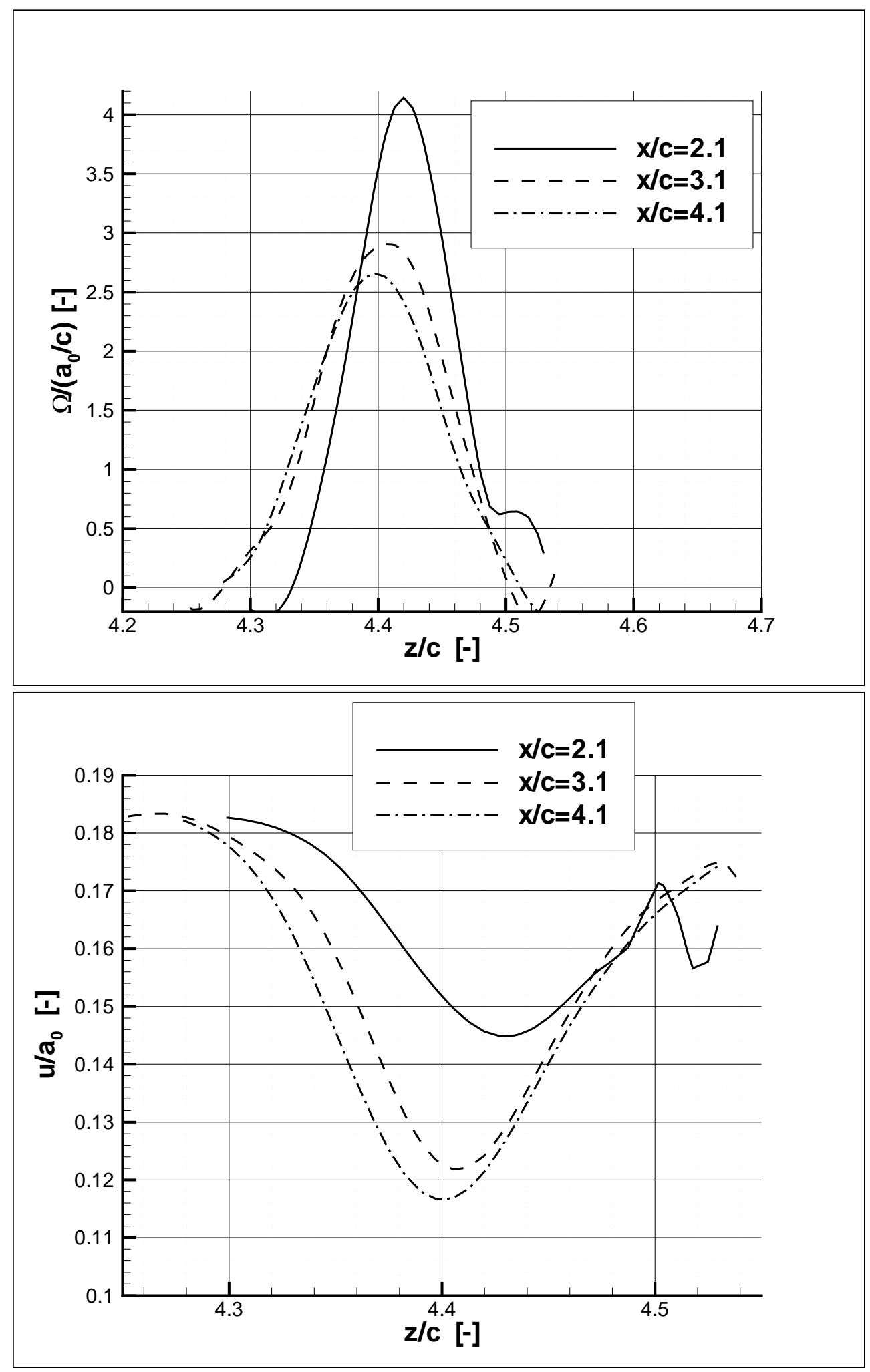

Figure 5.64: Vorticity $\Omega$ (top) and streamwise velocity $u$ (bottom) profiles vs. $z / c$ for pos $C, u_{\text {engine }} / u_{\infty}=1.77$ 

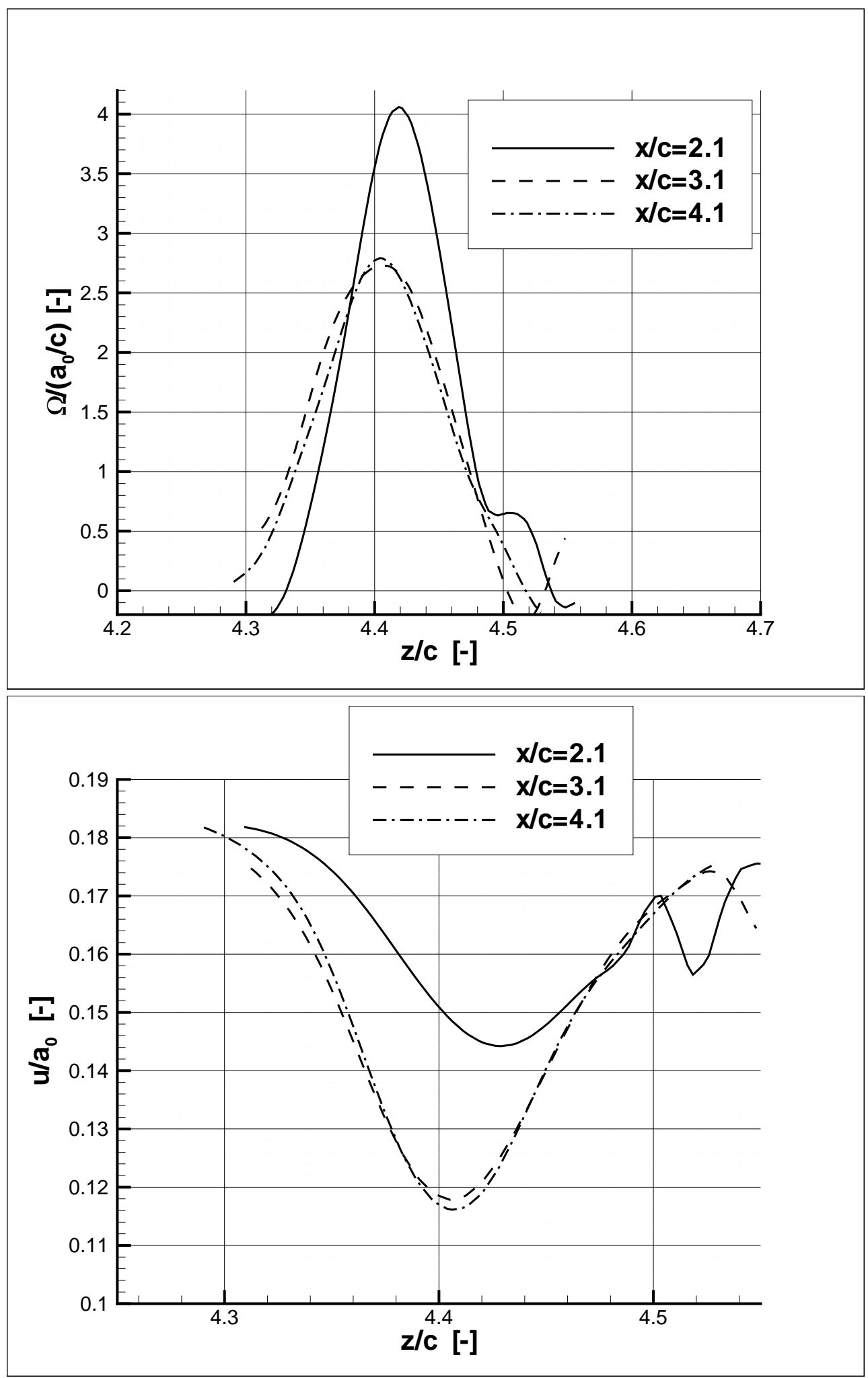

Figure 5.65: Vorticity $\Omega$ (top) and streamwise velocity $u$ (bottom) profiles vs. $z / c$ for posC-t2, $u_{\text {engine }} / u_{\infty}=2.87$ 


\section{Chapter 6}

\section{Conclusions}

"If the facts don't fit the theory, change the facts."

Albert Einstein.

The interaction between wingtip vortices and engine jets in the near wake is studied using numerical simulations of a rectangular wing/engine configuration. To achieve an accurate and efficient numerical solution of this flow problem several numerical issues such as discretization and acceleration techniques are addressed. Various numerical boundary conditions are also discussed and a new methodology for accurate boundary conditions suitable for inflow/outflow boundaries is proposed. The engine jet is simulated by prescribing mass conservative boundary conditions.

To calculate the turbulent wing and wake flow accurately and efficiently a turbulence model appropriate for near-wall and free shear turbulence is sought, which satisfies the constraints of the computational effort of a standard RANS formulation. Therefore, a new one-equation model based on the $k-\omega$ model is derived. Furthermore, a partial differential equation is derived to approximately calculate the wall distance that is required in most of the customary turbulence models. The iterative approximate solution of the wall distance equation is computationally more efficient than the exact calculation of the wall distance.

The validation of the numerical algorithm and the turbulence model is performed based on numerous analytic and experimentally investigated flows to ensure the generality of the numerical results. The findings show the newly proposed one-equation turbulence model to predict a wide range of flows especially jet and vortical flows more accurately than the SpalarT-Allmaras model and to be comparable to the quality even more efficient than the $k-\omega$ two-equation model.

Results of the numerical simulation of the flow around the rectangular wing/engine geometry defined in the collaborative research program SFB 401 at the RWTH AACHEN are presented for six configurations with different engine positions and engine jet velocities. The numerical approach is based on two steps. First, the flow field around the 
wing is determined and subsequently, the data of this simulation is used to provide the inflow condition for a separate wake simulation. The investigation shows an influence of the engine jet position on the vortex formation at the wingtip. The subsequent wake flow simulation discusses the spatial development of the three relevant flow structures in the near wake: the shear layer, the jet and the wingtip vortex. The interaction between the jet and the shear layer is unsteady and indicates instabilities that are generally amplified through the higher jet velocity and slightly influenced by the distance to the wingtip vortex. The wingtip vortex dynamics is almost identical for all simulated cases. Pronounced quantitative differences are found only in the velocity deficit within the vortex core for the engine position nearest the wingtip. This tendency is expected to be valid not only for the simulated near wake but also for the far wake.

The numerical simulations point out different crucial computational limitations to the three-dimensional wake flow simulation which are determined by vortex resolution, boundary conditions and turbulence modeling. The effort invested in this study to account for these factors represents only the first step to decrease the required computational resources. The vision of efficiently and accurately predicting the wake up to many wingspans downstream of the wing can only be achieved by implementing e.g. local adaptive refinement concepts. Furthermore, the findings indicate the impact of unsteady phenomena for the wake flow analysis, which have to be considered in future works. 


\section{References}

[1] B. S. Baldwin, H. Lomax. Thin Layer Approximation and Algebraic Model for Separated Turbulent Flows. AIAA Paper, 78-257,1978.

[2] B. S. Baldwin, T. J. Barth. A One-Equation Turbulence Transport Model for High Reynolds Number Wall-Bounded Flows. AIAA Paper, 91-0610,1991.

[3] J. E. Bardina, P. G. Huang, T. J. Coakly. Turbulence Modeling Validation, Testing and Development. NASA Technical Memorandum, 110446, 1997.

[4] G. K. Batchelor. Axial Flow in Trailing Line Vortices. Journal of Fluid Mechanics, vol.20, pp.645-658, 1964.

[5] H. Blasius. Grenzschichten in Flüssigkeiten mit kleiner Reibung. Z. Math. Physik, vol.56. pp.1-37, 1908.

[6] J. Boussinesq. Théorie de l'Écoulent Tourbillant. Mem. Présentés par Divers Savants Acad. Sci. Inst. Fr., vol.23, pp.46-50, 1877.

[7] J. S. Bradbury. The structure of a self-preserving turbulent plane jet. Journal of Fluid Mechanics, vol.23, pp.31-64, 1965.

[8] P. Bradshaw, D. H. Ferriss, N. P. Atwell N.P. Calculation of boundary layer development using the turbulent energy equation. Journal of Fluid Mechanics, vol.23, pp.31-64, 1976.

[9] A. Brandt. Multi-level adaptive solutions to boundary-value problems. Mathematics of Computation, vol.31, no.138, pp.333-390, 1977.

[10] A. Brandt. Guide to multigrid development. In Lecture Notes in Mathematics, SpringerVerlag, Berlin, pp.220-312, 1981.

[11] J. S. Chow, G. G. Zilliac, P. Bradshaw. Mean and Turbulence Measurements in the Near Field of a Wingtip Vortex. AIAA Journal, vol.35, no.10, pp.1561-1567, 1997.

[12] P. H. Cook, M. A. McDonald, M. C. P. Firmin. Aerofoil RAE 2822 - Pressure distributions, and boundary layer and wake measurements. AGARD AR-138, 1979.

[13] J. D. Crouch. Instability and transient growth for two trailing-vortex pairs. Journal Fluid Mechanics, vol.350, pp.311-330, 1997.

[14] J. D. Crouch, G. D. Miller, P. R. Spalart. Active-Control System for Breakup of Airplane Trailing Vortices. AIAA Journal, vol.39, no.12, pp.2374-2381, 2001.

[15] S. C. Crow. Stability theory for a pair of trailing vortices. AIAA Journal, vol.8, no.12, pp.2172-2179, 1970. 
[16] J. Dacles-Mariani, G. G. Zilliac, J. S. Chow, P. Bradshaw. Numerical/Experimental Study of a Wingtip Vortex in the Near Field. AIAA Journal, vol.33, no.9, pp.1561-1568, 1995.

[17] W. J. Devenport, M. C. Rife, S. I. Liapis, G. J. Follin. The structure and development of a wing-tip vortex. Journal Fluid Mechanics, vol.312, pp.67-106, 1996.

[18] P. Durbin. A Reynolds stress model for the near-wall turbulence. Journal of Fluid Mechanics, vol.249, pp.465ff, 1993.

[19] H. W. Emmons. Shear Flow Turbulence. Proceedings of the $2^{\text {nd }}$ U.S. Congress of Applied Mechanics, ASME, 1954.

[20] D. Enskog. Kinetische Theorie der Vorgänge in mässig verdünnten Gasen Dissertation, Uppsala, 1917.

[21] L. Euler. Principes généraux du mouvement des fluides. Hist. de l'Acad. de Berlin, 1755 .

[22] F. Eulitz. Numerische Simulation und Modellierung der instationären Strömung in Turbomaschinen. Dissertation, DLR, Köln, 2000.

[23] D. Fabre, L. Jacquin. Stability of a four-vortex aircraft wake model. Physics of Fluids, vol.12, no.10, pp.2438-2443, 2000.

[24] A. Fage, V. M. Falkner. Note on Experiments on the Temperature and Velocity in the Wake of a Heated Cylidrical Obstacle. Proc. Roy. Soc. (London), ser. A, vol.135, pp.702-705, 1932.

[25] E. Fares, M. Meinke, W. Schröder. Numerical Simulation of the Interaction of Wingtip Vortices and Engine jets in the Near Field. AIAA Paper, 2000-2222, 2000.

[26] E. Fares, M. Meinke, W. Schröder. Numerical Simulation of the Interaction of Flap Side-Edge Vortices and Engine Jets. ICAS 2000 Congress Proceedings, ICA0212 , 2000.

[27] E. Fares, W. Schröder. Numerical Simulation of Wake Flows. ECCOMAS CFD 2001 Congress Proceedings, 2001.

[28] E. Fares, W. Schröder. A Differential Equation for Approximate Wall Distance. International Journal for Numerical Methods in Fluids, vol.39, no.8, pp.743-762, 2002.

[29] S. Fell. Formierung und Struktur von Randwirbeln verschiedener Flügelkonfigurationen. Dissertation, ILR, RWTH Aachen, VDI Verlag, Reihe 7, Nr. 279, 1995.

[30] J. H. Ferziger, M. Perić. Computational Methods for Fluid Dynamics. Springer Verlag, Berlin/Heiderlberg, second edition, 1999.

[31] K. Gersten, H. Herwig. Strömungsmechanik. Grundlagen der Impuls-, Wärme und Stoffübertragung aus asymptotischer Sicht. Vieweg-Verlag, Braunschweig/Wiesbaden, 1992.

[32] T. Gerz, T. Ehret. Wake Dynamics and Exhaust Distribution behind Cruising Aircraft. AGARD CP-584, pp.35.1-35.12, 1996.

[33] P. Godin, D. W. Zingg. High-Lift Aerodynamic Computations with One- and TwoEquation Turbulence Models. AIAA Journal, vol.35, no.2, pp.238-243, 1997. 
[34] S. K. Godunov. A difference scheme for numerical computation of discontinuous solution of hydrodynamic equations. Math. Sbornik, vol.47, pp.271-306, 1959.

[35] S. P. Govindarajiu, P. G. Saffman. Flow in a turbulent trailing vortex. Physics of Fluids, vol.14, pp.2074-2080, 1971.

[36] F. Grasso, S. Pirozzoli, T. B. Gatski. Analysis and simulation of a turbulent, compressible starting vortex. Physics of Fluids, vol.11, no.2, pp.356-367, 1999.

[37] W. Gropp, E. Lusk, N. Doss, A. Skjellum A high-performance, portable implementation of the MPI Message-Passing Interface standard. Parallel Computing, vol.22, no.6, pp.789-828, 1996.

[38] B. Gustafsson, A. Sandström. Incompletely Parabolic Problems in Fluid Dynamics. SIAM J. Appl. Math., vol.35, part 2, pp.343-357, 1978.

[39] G. Heskestad. Hot-Wire Measurements in a Plane Turbulent Jet. Journal of Applied Mechanics, vol.32, no.4, pp.721-734, 1965.

[40] C. Hirsch. Numerical Computation of Internal and External Flows. John Wiley E Sons Ltd, vol.1, 1994.

[41] C. Hirsch. Numerical Computation of Internal and External Flows. John Wiley $\& 5$ Sons Ltd, vol.2, 1994.

[42] E. R. Hoffmann, P. N. Joubert. Turbulent line vortices. Journal of Fluid Mechanics, vol.16, pp.395-411, 1963.

[43] K. Hünecke. Vortex Wakes from Large Aircraft - A Challenge for Industrial Research. AIAA Paper, 2000-2216, 2000.

[44] G. Huppertz, E. Fares, R. Abstiens, W. Schröder. Investigation of Engine Jet/Wing-Tip Vortex Interference. DGLR Conference Proceedings, Stuttgart, 2002.

[45] L. Jacquin, F. Garnier. On the Dynamics of Engine Jets behind a Transport Aircraft. AGARD CP-584, pp.37.1-37.8, 1996.

[46] L. Jacquin, D. Fabre, P. Geffory. The Prope of a Transport Aircraft Wake in the Extended Near Field: an Experimental Study. AIAA Paper, 2001-1038, 2001.

[47] A. Jameson, W. Schmidt, E. Turkel. Numerical simulation of the Euler equations by finite volume methods using Runge-Kutta time stepping schemes. AIAA Paper, 81-1259, 1981.

[48] W. P. Jones, B. E. Launder. The predicition of laminarization with a two-equation model of turbulence. International Journal of Heat and Mass Transfer, vol.15, pp.301$314,1972$.

[49] M. R. Khorrami, B. A. Singer, R. H. Radeztsky, Jr. Reynolds Averaged Navier-Stokes Computations of a Flap Side-Edge Flow Field. AIAA Paper, 98-0768, 1998.

[50] S. J. Kline, M. V. Morkovin, G. Sovran, D. J. Cockrell. Computation of Turblent Boundary Layers. 1968 AFOSR-IFP-Stanford Conference Proceedings, vol.I, Thermoscience Division, Stanford University, CA, 1969.

[51] B. E. Launder, G. J. Reece, W. Rodi. Progress in the development of a Reynolds-Stress Turbulence Closure. Journal of Fluid Mechanics, vol.68, pt. 3,pp.537-566, 1975. 
[52] P. D. Lax, B. Wendroff. Systems of conservation laws. Comm. Pure and Applied Mathematics, vol.13, pp.217-237, 1960.

[53] P. D. Lax, B. Wendroff. Difference schemes for hyperbolic equations with high order of accuracy. Comm. Pure and Applied Mathematics, vol.17, pp.381-398, 1964.

[54] B. van Leer. Towards the Ultimate Conservative Difference Scheme V. A Second-Order. Journal of Computational Physics, vol.32, pp.101-136, 1979.

[55] T. Leweke, C. H. K. Williamson. Cooprative elliptic instability of a vortex pair. Journal of Fluid Mechanics, vol.360, pp.85-119, 1998.

[56] D. C. Lewellen, W. S. Lewellen. Large-Eddy Simulations of the Vortex-Pair Breakup in Aircraft Wakes. AIAA Journal, vol.34, no.11, pp.2337-2345, 1996.

[57] H. W. Liepmann, J. Laufer. Investiations of Free Turbulent Mixing. NACA TN, 1257, 1947.

[58] M. S. Liou, Ch. J. Steffen Jr. A New Flux Splitting Scheme. Journal of Computational Physics, vol.107, pp.23-39, 1993.

[59] H. Ludwieg, W. Tillmann. Untersuchungen über die Wandschubspannung in turbulenten Reibungsschichten. Ing.-Archiv, vol. 17, pp.288-299, 1949.

[60] M. Meinke, D. Hänel. W. Hackbusch(Ed.) Time Accurate Multigrid Solutions of the Navier-Stokes Equations. In Multigrid Methods III, Birkhäuser Verlag, pp.289-300, 1991.

[61] G. L. Mellor, H. J. Herring. Two Methods of calculating turbulent boundary layer behavior based on numerical solution of the equation of motion. Proc. Conf. Turb. Boundary Layer Pred., Stanford, 1968.

[62] F. R. Menter. Influence of Freestream Values on $k-\omega$ Turbulence Model Predictions. AIAA Journal, vol.30, no.6, pp.1657-1659, 1992.

[63] F. R. Menter. Zonal Two Equation $k$ - $\omega$ Turbulence Models for Aerodynamic Flows. AIAA Paper, 93-2906,1993.

[64] F. R. Menter. Eddy Viscosity Transport Equations and their Relation to the $k-\varepsilon$ Model. NASA-TM, 108854,1994.

[65] I. R. M. Moir. Measurements on a Two-dimensional Aerofoil with High-Lift Devices. AGARD AR-303, vol.2, pp.58-59, 1994.

[66] M. V. Morkovin. A. Favre(Ed.). Effects of compressibility on turbulent flows. in Méchanique de la Turbulence, Centre National de la Recherche Scientific, Paris, pp.367$380,1962$.

[67] A. J. Musker. Explicit expression for the smooth wall velocity distribution in a turbulent boundary layer. AIAA Journal, vol.17, pp.655-657, 1979.

[68] Y. Nagano, C. Q. Pei, H. Hattori. A New Low-Reynolds-Number One-Equation Model of Turbulence. Flow, Turbulence and Combustion, vol.63, pp.135-151, 1999.

[69] M. Navier. Mémoire sur les Lois du Mouvement des Fluides Mém, de l'Acad. des Sciences, vol.6, 1827. 
[70] V. W. Nee, L. S. G. Kovasznay. The Calculation of the Incompressible Turbulent Boundary Layer by a Simple Theory. Physics of Fluids, vol.12, pp.473ff, 1968.

[71] E. Özger, I. Schell, D. Jacob. On the Structure and Attenuation of an Aircraft Wake AIAA Paper, 2000-4127, 2000.

[72] E. Özger. Abschwächung des Wirbelnachlaufs von Flugzeugen mit Hilfe von Finnen. Dissertation, ILR, RWTH Aachen, VDI Verlag, Reihe 7, Nr. 422, 2001.

[73] C. Pantano, L. Jacquin. Differential Rotation Effects within a turbulent batchelor vortex. Direct and Large-Eddy Simulation-IV Workshop Proceedings, Enschede, The Netherlands, 2001.

[74] W. R. C. Phillips. The Turbulent Trailing Vortex during Roll-Up. Journal of Fluid Mechanics, vol.105, pp.451-467, 1981.

[75] W. R. C. Phillips, J. A. H. Graham. Reynolds stress measurements in a turbulent trailing vortex. Journal of Fluid Mechanics, vol.147, pp.451-467, 19814

[76] L. Prandtl. Über die ausgebildete Turbulenz. ZAMM, vol.5, pp.136-139, 1925.

[77] L. Prandtl. Über ein neues Formelsystem für die ausgebildete Turbulenz. Nachr. Akad. Wiss. Göttingen, Math. Phys., pp.6-19, 1945.

[78] S. D. Poisson. Mémoire sur les Équations générales de l'equilibre et du Mouvement des Corps solides élastique et des Fluides. Journal de l'École Polytechnique, vol.8 , 1831.

[79] T. J. Poinsot, S. K. Lele. Boundary Conditions for Direct Simulations of Compressible Viscous Flows. Journal of Computational Physics, vol.101. pp.104-129, 1992.

[80] S. B. Pope. An Explanation of the Turbulent Round-Jet/Plane-Jet Anomaly. AIAA Journal, vol.16, no.3, pp.279-281, 1978.

[81] J. H. Qin. Numerical Simulations of a Turbulent Axial Vortex. PhD Thesis, Purdue University, 1998.

[82] T. R. Quackenbush, M. E. Teske, A. J. Bilanin. Dynamics of Exhaust Plume Entrainment in Aircraft Vortex Wakes. AIAA Paper, 96-0747, 1996.

[83] R. H. Radeztsky, B. A. Singer, M. R. Khorrami. Detailed Measurements of a Flap Side-Edge Flow Field. AIAA Paper, 98-0700, 1998.

[84] R. D. Richtmyer, K. W. Morton. Difference Methods for Initial Value Problems. WileyInterscience, London, second edition, 1967.

[85] T. Rister. Grobstruktur-Simulation schwach kompressibler turbulenter Freistrahlen ein Vergleich zweier Lösungsansätze. Dissertation, AIA, RWTH Aachen, Shaker Verlag, 1998.

[86] O. Reynolds. On the Extent and Action of the Heating Surface for Steam Boilers. Proc. Manchester Lit. Phil. Soc., vol.14, pp.7-12, 1874.

[87] J. J. Robinson. A Simulation-Based Study of the Impact of Aircraft Wake Turbulence Weight Categories on Airport Capacity. AGARD CP-584, pp.22.1-22.15, 1996.

[88] W. Rodi. A New Method for Analyzing How-Wire Signals in Highly Turbulent Flows and its Evaluation on Round Jets. Disa Information, no.17, 1975. 
[89] P. L. Roe. A survey of Upwind Differencing Techniques. In Lect. Notes in Phys., Springer-Verlag, vol.323, pp.69ff, 1989.

[90] S. E. Rogers, F. Menter, P. A. Durbin, N. M. Mansour. A Comparison of Turbulence Models in Computing Multi-Element Airfoil Flows. AIAA Paper, 94-0291, 1994.

[91] L. Rosenhead. The Formation of Vortices from a Surface of Discontinuity. Proc. Roy. Soc. (London), vol.134, pp.170-192, 1931.

[92] V. J. Rossow. Lift-generated vortex wakes of subsonic transport aircraft. Progress in Aerospace Sciences, vol.35, pp.507-660, 1999.

[93] C. L. Rumsey, V. N. Vasta. A Comparison of the Predictive capabilities of Several Turbulence Models using Upwind and Central-Difference Computer Codes. AIAA Paper, 93-0192, 1993.

[94] R. Rudnik, A. Ronzheimer, C. C. Rossow, H. Hoheisel. Investigation of the Flowfield around an Isolated Engine with Fan and Core Jet. AGARD CP-498, pp.29.1-29.8, 1992.

[95] P. G. Saffman. Structure of turbulent line vortices. Physics of Fluids, vol.16, no.8, pp.1181-1188, 1973.

[96] P. G. Saffman. Vortex Dynamics. Cambridge Monographs on Mech. and Appl.Math., Cambridge, 1992.

[97] A. E. Samuel, P. N. Joubert. A boundary layer developing in an increasingly adverse pressure gradient. Journal of Fluid Mechanics, vol.66, pp.481-505, 1974.

[98] N. Satofuka, K. Morinishi, T. Takami, A. Shimizu. Computation of Two-Dimensional Transonic Cascade Flow using a new Navier-Stokes Solver. AIAA paper, 86-1381,1986.

[99] V. A. Sai, F. M. Lutfy. Analysis of the Baldwin-Barth and Spalart-Allmaras OneEquation Turbulence Models. AIAA Journal, vol.33, no.10, pp.1971-1974, 1995.

[100] R. S. Scorer, L. J. Davenport. Contrails and aircraft downwash. Journal of Fluid Mechanics, vol.43, pp.451-464, 1970.

[101] A. N. Secundov. Application of the Differential Equation for Turbulent Viscosity to the Analysis of Plane Nonself-Similar Flows. Akademiya Nauk, SSSR, Izvestiia, Mekhanika Zhidkosti i Gaza, pp.114-127, 1971.

[102] A. N. Secundov. personal communication, 2000.

[103] G. Seider. Numerische Untersuchung Transsonischer Strömungen. Dissertation, AIA, RWTH Aachen, 1991.

[104] L. I. Schiff. Quantum Mechanics. McGraw-Hill, 3rd edn., 1968.

[105] H. Schlichting, E. Truckenbrodt. Aerodynamik des Flugzeuges. Part II. SpringerVerlag, Berlin/Heidelberg, 2. edition, 1969.

[106] H. Schlichting, K. Gersten. Grenzschicht-Theorie. Springer-Verlag, Berlin/Heidelberg, 9. edition, 1997.

[107] W. Schröder. Numerische Integration der Navier-Stokes Gleichungen unter Verwendung des Mehrgitterkonzeptes. Dissertation, AIA, RWTH Aachen, 1987. 
[108] G. B. Schubauer, P. S. Klebanoff. Contributions on the mechanics of boundary layer transition. NASA-TN, 3489, 1955, also NASA-TR, 1289, 1956.

[109] M. Shur, M. Strelets, L. Zaikov, A. N. Gulyaev, V. E. Kozlov, A. N. Secundov. A Comparative numerical testing of one- and two-equation turbulence models for flows with separation and reattachment. AIAA Paper, 95-0863, 1995.

[110] P. R. Spalart, S. R. Allmaras. A One-Equation Turbulence Model for Aerodynamic Flows. AIAA Paper, 92-0439, 1992.

[111] P. R. Spalart, M. Shur. On the Sensitization of Turbulence Models to Rotation and Curvature. Aerospace Science and Technology, no.5, pp.297-302, 1997.

[112] P. R. Spalart. Airplane Trailing Vortices. Ann. Rev. Fluid Mechanics, vol.30, pp.107138, 1998.

[113] P. R. Spalart. personal communication, 2000.

[114] P. R. Spalart. Trends in Turbulence Treatments. AIAA Paper, 2000-2306, 2000.

[115] P. R. Spalart, M. Kh. Strelets, A. K. Travin, M. L. Shur. Modeling the Turbulent Vortex Wake Behind a High-Lift Wing. Fluid Dynamics, vol.36, no.5, pp.726-733, 2001.

[116] D. B. Spalding. A single formula for the "law of the wall". ASME J. Appl. Mech., vol.28, pp.455-457, 1961.

[117] M. Sreedhar, S. Ragab. Large eddy simulation of longitudinal stationary vortices. Physics of Fluids, vol.6, pp.2501-2514, 1994.

[118] T. E. Stanton, J. R. Pannell. Similarity of Motion in Relation to the Surface Friction of Fluids. Trans. R. Soc. (London), ser. A, vol.214, pp.199-224, 1914.

[119] K. Stewartson. Correlated Incompressible and Compressible Boundary Layers. Proc. Roy. Soc. (London), ser. A, vol.200, pp.84-100, 1949.

[120] G. N. Stokes. On the Theories of the Internal Friction of Fluids in Motion. Math. and Phys. Papers, I, 1845.

[121] E. Stumpf, R. Rudnik, A. Ronzheimer. Euler Computation of the Nearfild Wake Vortex of an Aircraft in Take-Off Configuration. Aerosp, Sci.ETTech., no.4, pp.535-543, 2000.

[122] H. Tennekes, J. L. Lumley. First Course in Turbulence. The MIT Press, 1972.

[123] J. F. Thompson, Z. U. A. Warsi, C. W. Mastin. Numerical Grid Generation, Foundations and Applications. North-Holland, Amsterdam, 1985.

[124] K. W. Thompson. Time Dependent Boundary Conditions for Hyperbolic Systems II. Journal of Computational Physics, vol.89, pp.439-461, 1990.

[125] A. A. Townsend. The Structure of Turbulent Shear Flow. Cambridge University Press, Cambridge, England, second ed., 1976.

[126] P. G. Tucker. Assessment of geometric multilevel convergence robustness and a wall distance method for flows with multiple internal boundaries. Applied Mathematical Modelling, vol.22, pp.293-311, 1998.

[127] P. G. Tucker. Prediction of turbulent oscillatory flows in complex systems. International Journal for Numerical Methods in Fluids, vol.33, pp.869-895, 2000. 
[128] P. G. Tucker. personal communication, 2001.

[129] Y. R. Tsai. Rapid and Accurate Computation of the Distance Function Using Grids. Journal of Computational Physics, vol.178, pp.175-195, 2002.

[130] G. Van Albada, B. Van Leer, J. Robers. A Comparative Study of Computational Methods in Cosmic Gas Dynamics. Astron. Astrophysics, vol.108, pp.76-84, 1982.

[131] E. R. Van Driest. Turbulent Boundary Layer in Compressible Fluids. Journal of the Aeronatical Sciences, vol.18, pp.145-160, 1951.

[132] Y. Wada, M. S. Liou. A Flux Splitting Scheme with High-Resolution and Robustness for Discontinuities. AIAA Paper, 94-0083, 1994.

[133] A. Wambecq. Rational Runge-Kutta Methods for Solving Systems of Ordinary Differential Equations. Computing, vol.20, pp.333-342, 1978.

[134] Q. Wang, S. J. Massey, K. S. Abdol-Hamid. Solving Navier-Stokes Equations with Advanced Turbulence Models on Three-Dimensional Unstructured Grids. AIAA Paper, 99-0156, 1999.

[135] F. L. Westwater. The Rolling up of the Surface of Discontinuity behind an Airfoil of Finite Span. Aeronautical Research Council, Repts. and Memo., London, no.1692, pp.116-131, 1935.

[136] J. H. Weygandt, R. D. Mehta. Three-Dimensional Structure of Straight and Curved Plane Wakes. Journal of Fluid Mechanics, vol.282, pp.279, 1995.

[137] S. E. Widnall. The structure and dynamics of vortex filaments. Ann. Rev. Fluid Mechanics, vol.7, pp.141-165, 1975.

[138] D. C. Wilcox. Reassessment of the scale-determining equation for advanced turbulence models. AIAA Journal, vol.26, no.11, pp.1299-1310, 1988.

[139] D. C. Wilcox. Turbulence Modeling for CFD. DCW Industries, second ed., 1998.

[140] I. Wygnanski, H. E. Fiedler. Some Measurements in Self-Presering Jet. Journal of Fluid Mechanics, vol.38, pp.577-612, 1969.

[141] O. Zeman. The presistence of trailing vortices: A modeling study. Physics of Fluids, vol.7, no.1, pp.135-143, 1995. 


\section{Appendix A}

\section{Dimensionless Variables}

The equations are nondimensionalized based on the following reference values

$$
\begin{aligned}
& x=\frac{x^{*}}{L^{*}} \quad y=\frac{y^{*}}{L^{*}} \quad y=\frac{y^{*}}{L^{*}} \quad t=\frac{t^{*}}{L^{*} / a_{0}^{*}} \quad T=\frac{T^{*}}{T_{0}^{*}} \\
& u=\frac{u^{*}}{a_{0}^{*}} \quad v=\frac{v^{*}}{a_{0}^{*}} \quad w=\frac{w^{*}}{a_{0}^{*}} \quad \rho=\frac{\rho^{*}}{\rho_{0}^{*}} \quad p=\frac{p^{*}}{\rho_{0}^{*} a_{0}^{*^{2}}} \\
& e=\frac{e^{*}}{a_{0}^{*^{2}}} \quad \mu=\frac{\mu^{*}}{\mu_{0}^{*}} \quad \lambda=\frac{\lambda^{*}}{\lambda_{0}^{*}} \quad c_{p, v}=\frac{c_{p, v}^{*}}{c_{p, v_{0}}^{*}} \quad \nu_{t}=\frac{\nu_{t}^{*}}{\mu_{0}^{*} / \rho_{0}^{*}}
\end{aligned}
$$

that lead to the characteristic parameters:

- REYNOLDS number: $\quad R e_{0}=\frac{\rho_{0}^{*} a_{0}^{*} L^{*}}{\mu_{0}^{*}}$

- Prandtl number: $\quad \operatorname{Pr}_{0}=\frac{\mu_{0}^{*} c_{p_{0}}^{*}}{\lambda_{0}^{*}} \quad P r_{0}=0.72$ for air

$$
\left(P r_{t}=0.9 \text { for turbulent flows }\right)
$$

- ratio of specific heats: $\quad \gamma_{0}=\frac{c_{p_{0}}^{*}}{c_{v 0}^{*}} \quad \gamma_{0}=1.4$ for air

Given the classical definition of $R e_{\infty}=\frac{\rho_{\infty}^{*} u_{\infty}^{*} L^{*}}{\mu_{\infty}^{*}}$ and $M a_{\infty}=\frac{u_{\infty}^{*}}{a_{\infty}^{*}}$ the REYNOLDS number $R e_{0}$ based on the stagnation values can be calculated as follows

$$
\begin{aligned}
& T_{\infty}=\frac{T_{\infty}^{*}}{T_{0}^{*}}=\left(1+\frac{\gamma_{0}-1}{2} M a_{\infty}^{2}\right)^{-1} \quad \rho_{\infty}=\frac{\rho_{\infty}^{*}}{\rho_{0}^{*}}=T_{\infty}^{\frac{1}{\gamma_{0}-1}} \\
& u_{\infty}=\frac{u_{\infty}^{*}}{a_{0}^{*}}=\frac{u_{\infty}^{*}}{a_{\infty}^{*}} \sqrt{\frac{T_{\infty}^{*}}{T_{0}^{*}}}=M a_{\infty} \sqrt{T_{\infty}} \quad \mu_{\infty}=\frac{\mu_{\infty}^{*}}{\mu_{0}^{*}}=T_{\infty}^{0.72} \\
& R e_{0}=\frac{\rho_{0}^{*} a_{0}^{*} L^{*}}{\mu_{0}^{*}}=\frac{\frac{\rho_{\infty}^{*} u_{\infty}^{*} L^{*}}{\mu_{\infty}^{*}} \cdot \frac{\mu_{\infty}^{*}}{\mu_{0}^{*}}}{\frac{\rho_{\infty}^{*}}{\rho_{0}^{*}} \cdot \frac{u_{\infty}^{*}}{a_{\infty}^{*}} \cdot \sqrt{\frac{T_{\infty}^{*}}{T_{0}^{*}}}}=\frac{R e_{\infty} \cdot T_{\infty}^{0.72}}{\rho_{\infty} \cdot M a_{\infty} \cdot \sqrt{T_{\infty}}}
\end{aligned}
$$




\section{Appendix B}

\section{Averaging Rules}

Based on the definitions of REYNOLDS averaging in equation (2.14) and FAVRE averaging in equation (2.16) several averaging rules are derived

$$
\begin{aligned}
\overline{(\Phi+\Psi)} & =\bar{\Phi}+\bar{\Psi} \\
\overline{\Phi \cdot \bar{\Psi}} & =\bar{\Phi} \cdot \bar{\Psi} \neq \overline{\Phi \cdot \Psi} \\
\overline{\frac{\partial \Phi}{\partial \xi}} & =\frac{\partial \bar{\Phi}}{\partial \xi} \\
\overline{\rho \Phi} & =\bar{\rho} \\
\overline{\Phi^{\prime}} & =0 \\
\overline{\rho \Phi^{\prime \prime}} & =0 \\
\overline{\Phi^{\prime \prime}} & =-\frac{\overline{\rho^{\prime} \Phi^{\prime \prime}}}{\rho}=-\frac{\frac{\rho^{\prime} \Phi^{\prime}}{\bar{\rho}}}{\rho} \neq 0 \\
\widetilde{\Phi^{\prime \prime} \Psi^{\prime \prime}} & =\overline{\Phi^{\prime} \Psi^{\prime}}-\frac{\overline{\rho^{\prime} \Phi^{\prime} \rho^{\prime} \Psi^{\prime}}}{\bar{\rho}^{2}}+\frac{\overline{\rho^{\prime} \Phi^{\prime} \Psi^{\prime}}}{\bar{\rho}} .
\end{aligned}
$$




\section{Appendix $\mathrm{C}$}

\section{Equations in Cartesian Coordinates}

The complete dimensionless and FAVRE averaged conservation equations for mass, momentum and energy is extended to include the proposed one-equation turbulence model in a conservation like form of $\bar{\rho} \check{\nu}$

$$
\frac{\partial \vec{Q}}{\partial t}+\frac{\partial \vec{F}_{1}}{\partial x}+\frac{\partial \vec{F}_{2}}{\partial y}+\frac{\partial \overrightarrow{F_{3}}}{\partial z}=\vec{S}
$$

with the vector $\vec{Q}$ of the conservative variables

$$
\vec{Q}=\left(\begin{array}{c}
\bar{\rho} \\
\bar{\rho} \tilde{u} \\
\bar{\rho} \tilde{v} \\
\bar{\rho} \tilde{w} \\
\bar{\rho} \tilde{e} \\
\bar{\rho} \tilde{v}
\end{array}\right)
$$

and the flux vectors $\vec{F}_{1}, \vec{F}_{2}, \vec{F}_{3}$

$$
\begin{aligned}
& \vec{F}_{1}=\vec{F}_{A_{1}}-\operatorname{Re}_{0}^{-1} \vec{F}_{D_{1}}=\left(\begin{array}{c}
\bar{\rho} \tilde{u} \\
\bar{\rho} \tilde{u}^{2}+\bar{p} \\
\bar{\rho} \tilde{u} \tilde{v} \\
\bar{\rho} \tilde{u} \tilde{w} \\
\tilde{u}(\bar{\rho} \tilde{e}+\bar{p}) \\
\bar{\rho} \tilde{\nu} \tilde{u}
\end{array}\right)-\frac{1}{R e_{0}}\left(\begin{array}{c}
0 \\
\tau_{x x} \\
\tau_{x y} \\
\tau_{x z} \\
\tilde{u} \tau_{x x}+\tilde{v} \tau_{x y}+\tilde{w} \tau_{x z}+q_{x} \\
(\bar{\mu}+\sigma \bar{\rho} \tilde{\nu}) \cdot \frac{\partial \tilde{\nu}}{\partial x}
\end{array}\right) \\
& \vec{F}_{2}=\vec{F}_{A_{2}}-R e_{0}^{-1} \vec{F}_{D_{2}}=\left(\begin{array}{c}
\bar{\rho} \tilde{v} \\
\bar{\rho} \tilde{v} \tilde{u} \\
\bar{\rho} \tilde{v}^{2}+\bar{p} \\
\bar{\rho} \tilde{v} \tilde{w} \\
\tilde{v}(\bar{\rho} \tilde{e}+\bar{p}) \\
\bar{\rho} \tilde{\nu} \tilde{v}
\end{array}\right)-\frac{1}{R e_{0}}\left(\begin{array}{c}
0 \\
\tau_{y x} \\
\tau_{y y} \\
\tau_{y z} \\
\tilde{u} \tau_{y x}+\tilde{v} \tau_{y y}+\tilde{w} \tau_{y z}+q_{y} \\
(\bar{\mu}+\sigma \bar{\rho} \tilde{\nu}) \cdot \frac{\partial \tilde{\nu}}{\partial y}
\end{array}\right) \\
& \vec{F}_{3}=\vec{F}_{A_{3}}-R e_{0}^{-1} \vec{F}_{D_{3}}=\left(\begin{array}{c}
\bar{\rho} \tilde{w} \\
\bar{\rho} \tilde{w} \tilde{u} \\
\bar{\rho} \tilde{w} \tilde{v} \\
\bar{\rho} \tilde{w}^{2}+\bar{p} \\
\tilde{w}(\bar{\rho} \tilde{e}+\bar{p}) \\
\bar{\rho} \check{\nu} \tilde{w}
\end{array}\right)-\frac{1}{R e_{0}}\left(\begin{array}{c}
0 \\
\tau_{z x} \\
\tau_{z y} \\
\tau_{z z} \\
\tilde{u} \tau_{z x}+\tilde{v} \tau_{z y}+\tilde{w} \tau_{z z}+q_{z} \\
(\bar{\mu}+\sigma \bar{\rho} \bar{\nu}) \cdot \frac{\partial \tilde{v}}{\partial z}
\end{array}\right)
\end{aligned}
$$


and the vector of source terms $\vec{S}$

$$
\vec{S}=\left(\begin{array}{c}
0 \\
0 \\
0 \\
0 \\
0 \\
(1-\alpha)\left[\frac{\bar{\rho} \check{\nu}}{\omega} P-\frac{2}{3} \bar{\rho} \check{\nu} \nabla \vec{u}\right]-\left(\beta^{*}-\beta\right) \bar{\rho} \check{\nu} \omega-\bar{\rho} k_{i n i t}\left(\phi \frac{\check{\nu}}{\nu_{\text {init }}}\right)^{5}+2 \frac{(\bar{\mu}+\sigma \bar{\rho} \check{\nu})}{R e_{0} \omega} C D_{\check{\nu} \omega}
\end{array}\right)
$$

$\nabla \vec{u}$ is the divergence of velocity

$$
\nabla \vec{u}=\frac{\partial \tilde{u}}{\partial x}+\frac{\partial \tilde{v}}{\partial y}+\frac{\partial \tilde{w}}{\partial z}
$$

and $P$ represents the term

$$
\begin{aligned}
P & =2\left(\frac{\partial \tilde{u}}{\partial x}\right)^{2}+2\left(\frac{\partial \tilde{v}}{\partial y}\right)^{2}+2\left(\frac{\partial \tilde{w}}{\partial z}\right)^{2} \\
& +\frac{\partial \tilde{u}}{\partial y}\left(\frac{\partial \tilde{u}}{\partial y}+\frac{\partial \tilde{v}}{\partial x}\right)+\frac{\partial \tilde{u}}{\partial z}\left(\frac{\partial \tilde{u}}{\partial z}+\frac{\partial \tilde{w}}{\partial x}\right)+\frac{\partial \tilde{v}}{\partial x}\left(\frac{\partial \tilde{v}}{\partial x}+\frac{\partial \tilde{u}}{\partial y}\right) \\
& +\frac{\partial \tilde{v}}{\partial z}\left(\frac{\partial \tilde{v}}{\partial z}+\frac{\partial \tilde{w}}{\partial y}\right)+\frac{\partial \tilde{w}}{\partial x}\left(\frac{\partial \tilde{w}}{\partial x}+\frac{\partial \tilde{u}}{\partial z}\right)+\frac{\partial \tilde{w}}{\partial y}\left(\frac{\partial \tilde{w}}{\partial y}+\frac{\partial \tilde{v}}{\partial z}\right)
\end{aligned}
$$

$C D_{\check{\nu} \omega}$ the cross diffusion term of the $\check{\nu}$ equation

$$
C D_{\check{\nu} \omega}=\left(\frac{\partial \check{\nu}}{\partial x} \frac{\partial \omega}{\partial x}+\frac{\partial \check{\nu}}{\partial y} \frac{\partial \omega}{\partial y}+\frac{\partial \check{\nu}}{\partial z} \frac{\partial \omega}{\partial z}\right)
$$

The stress tensor $\overline{\bar{\tau}}$ is defined by

$$
\overline{\bar{\tau}}=\left(\bar{\mu}+\mu_{t}\right)\left(\begin{array}{ccc}
\frac{2}{3}\left(2 \frac{\partial \tilde{u}}{\partial x}-\left(\frac{\partial \tilde{v}}{\partial y}+\frac{\partial \tilde{w}}{\partial z}\right)\right) & \frac{\partial \tilde{u}}{\partial y}+\frac{\partial \tilde{v}}{\partial x} & \frac{\partial \tilde{u}}{\partial z}+\frac{\partial \tilde{w}}{\partial x} \\
\frac{\partial \tilde{u}}{\partial y}+\frac{\partial \tilde{v}}{\partial x} & \frac{2}{3}\left(2 \frac{\partial \tilde{v}}{\partial y}-\left(\frac{\partial \tilde{u}}{\partial x}+\frac{\partial \tilde{w}}{\partial z}\right)\right) & \frac{\partial \tilde{v}}{\partial z}+\frac{\partial \tilde{w}}{\partial y} \\
\frac{\partial \tilde{u}}{\partial z}+\frac{\partial \tilde{w}}{\partial x} & \frac{\partial \tilde{v}}{\partial z}+\frac{\partial \tilde{w}}{\partial y} & \frac{2}{3}\left(2 \frac{\partial \tilde{w}}{\partial z}-\left(\frac{\partial \tilde{u}}{\partial x}+\frac{\partial \tilde{v}}{\partial y}\right)\right)
\end{array}\right)
$$

and the heat conduction $\vec{q}$ is given by

$$
\vec{q}=\frac{1}{\left(\gamma_{0}-1\right)}\left(\frac{\bar{\mu}}{P r_{0}}+\frac{\mu_{t}}{P r_{t}}\right)\left(\begin{array}{c}
\frac{\partial \bar{T}}{\partial x} \\
\frac{\partial \bar{T}}{\partial y} \\
\frac{\partial \bar{T}}{\partial z}
\end{array}\right)
$$

The turbulent viscosity $\mu_{t}$ is calculated according to

$$
\mu_{T}=\bar{\rho} \nu_{t}=\bar{\rho} \check{\nu} f_{v 1} \quad \text { with } \quad f_{v 1}=\frac{\chi^{3}}{\chi^{3}+c_{v 1}^{3}} \quad \text { and } \quad \chi=\frac{\check{\nu}}{\nu}
$$

Further functions needed for the turbulence model are

$$
\omega=\frac{\sqrt{\left(\frac{\partial \tilde{u}}{\partial y}+\frac{\partial \tilde{v}}{\partial x}\right)^{2}+\left(\frac{\partial \tilde{u}}{\partial z}+\frac{\partial \tilde{w}}{\partial x}\right)^{2}+\left(\frac{\partial \tilde{w}}{\partial y}+\frac{\partial \tilde{v}}{\partial z}\right)^{2}+2\left(\left(\frac{\partial \tilde{u}}{\partial x}\right)^{2}+\left(\frac{\partial \tilde{v}}{\partial y}\right)^{2}+\left(\frac{\partial \tilde{w}}{\partial z}\right)^{2}\right)}}{\sqrt{\beta_{c}^{*}}}
$$




$$
\begin{aligned}
\psi_{k}= & \max \left[0 ., \frac{\check{\nu}}{R e_{0} \omega^{3}}\left(\left(\frac{\partial \omega}{\partial x}\right)^{2}+\left(\frac{\partial \omega}{\partial y}\right)^{2}+\left(\frac{\partial \omega}{\partial z}\right)^{2}\right)\right. \\
& \left.+\frac{1}{R e_{0} \omega^{2}}\left(\frac{\partial \check{\nu}}{\partial x} \frac{\partial \omega}{\partial x}+\frac{\partial \check{\nu}}{\partial y} \frac{\partial \omega}{\partial y}+\frac{\partial \check{\nu}}{\partial z} \frac{\partial \omega}{\partial z}\right)\right] \\
\psi_{\omega}= & \left|\frac{\Omega_{i j} \Omega_{j k} S_{k i}}{\left(\beta_{0}^{*} \omega\right)^{3}}\right| \\
= & \frac{1}{\left(\beta_{0}^{*} \omega\right)^{3}} \mid 3 / 4\left(\tilde{v}_{z} \tilde{u}_{y} \tilde{w}_{x}+\tilde{w}_{y} \tilde{v}_{x} \tilde{u}_{z}\right) \\
& +1 / 2\left(\tilde{v}_{y} \tilde{v}_{x} \tilde{u}_{y}+\tilde{v}_{y} \tilde{w}_{y} \tilde{v}_{z}+\tilde{w}_{z} \tilde{w}_{x} \tilde{u}_{z}+\tilde{w}_{z} \tilde{w}_{y} \tilde{v}_{z}+\tilde{u}_{x} \tilde{w}_{x} \tilde{u}_{z}+\tilde{u}_{x} \tilde{v}_{x} \tilde{u}_{y}\right) \\
& -1 / 4\left(\tilde{v}_{y} \tilde{v}_{z}^{2}+\tilde{w}_{z} \tilde{w}_{x}^{2}+\tilde{w}_{z} \tilde{u}_{z}^{2}+\tilde{w}_{z} \tilde{w}_{y}^{2}+\tilde{w}_{z} \tilde{v}_{z}^{2}+\tilde{u}_{x} \tilde{v}_{x}^{2}\right. \\
& \quad+\tilde{u}_{x} \tilde{u}_{y}^{2}+\tilde{u}_{x} \tilde{w}_{x}^{2}+\tilde{u}_{x} \tilde{u}_{z}^{2}+\tilde{v}_{y} \tilde{v}_{x}^{2}+\tilde{v}_{y} \tilde{u}_{y}^{2}+\tilde{v}_{y} \tilde{w}_{y}^{2} \\
& \left.\quad+\tilde{v}_{z} \tilde{u}_{y} \tilde{u}_{z}+\tilde{v}_{z} \tilde{v}_{x} \tilde{w}_{x}+\tilde{v}_{z} \tilde{v}_{x} \tilde{u}_{z}+\tilde{w}_{y} \tilde{u}_{y} \tilde{w}_{x}+\tilde{w}_{y} \tilde{u}_{y} \tilde{u}_{z}+\tilde{w}_{y} \tilde{v}_{x} \tilde{w}_{x}\right)
\end{aligned}
$$




\section{Appendix D}

\section{Transformation to Curvilinear Coordinates}

The flow equations can be transformed from cartesian coordinates $x, y, z$ into general curvilinear coordinates $\xi, \eta, \zeta$ following the transformation formulas

$$
\vec{\xi}=\vec{\xi}(x, y, z) \quad \vec{x}=\vec{\xi}(\xi, \eta, \zeta)
$$

that allow a simpler discretization and implementation of boundary conditions as described in chapter 4. The transformations

$$
\left(\begin{array}{c}
\frac{\partial}{\partial x} \\
\frac{\partial}{\partial y} \\
\frac{\partial}{\partial z}
\end{array}\right)=\left(\begin{array}{ccc}
\xi_{x} & \eta_{x} & \zeta_{x} \\
\xi_{y} & \eta_{y} & \zeta_{y} \\
\xi_{z} & \eta_{z} & \zeta_{z}
\end{array}\right)\left(\begin{array}{c}
\frac{\partial}{\partial \xi} \\
\frac{\partial}{\partial \eta} \\
\frac{\partial}{\partial \zeta}
\end{array}\right) \quad\left(\begin{array}{c}
\frac{\partial}{\partial \xi} \\
\frac{\partial}{\partial \eta} \\
\frac{\partial}{\partial \zeta}
\end{array}\right)=\left(\begin{array}{ccc}
x_{\xi} & y_{\xi} & z_{\xi} \\
x_{\eta} & y_{\eta} & z_{\eta} \\
x_{\zeta} & y_{\zeta} & z_{\zeta}
\end{array}\right)\left(\begin{array}{c}
\frac{\partial}{\partial x} \\
\frac{\partial}{\partial y} \\
\frac{\partial}{\partial z}
\end{array}\right)
$$

map the derivatives from the cartesian space to a time independent curvilinear space and vice versa. Through comparison of the same derivatives of each transformation matrix the metric relations are derived

$$
\begin{array}{lll}
\xi_{x}=J^{-1}\left(y_{\eta} z_{\zeta}-y_{\zeta} z_{\eta}\right) & \eta_{x}=J^{-1}\left(y_{\zeta} z_{\xi}-y_{\xi} z_{\zeta}\right) & \zeta_{x}=J^{-1}\left(y_{\xi} z_{\eta}-y_{\eta} z_{\xi}\right) \\
\xi_{y}=J^{-1}\left(z_{\eta} x_{\zeta}-z_{\zeta} x_{\eta}\right) & \eta_{y}=J^{-1}\left(z_{\zeta} x_{\xi}-z_{\xi} x_{\zeta}\right) & \zeta_{y}=J^{-1}\left(z_{\xi} x_{\eta}-z_{\eta} x_{\xi}\right) \\
\xi_{z}=J^{-1}\left(x_{\eta} y_{\zeta}-x_{\zeta} y_{\eta}\right) & \eta_{z}=J^{-1}\left(x_{\zeta} y_{\xi}-x_{\xi} y_{\zeta}\right) & \zeta_{z}=J^{-1}\left(x_{\xi} y_{\eta}-x_{\eta} y_{\xi}\right)
\end{array}
$$

with $J$ as the determinant of the transformation matrix

$$
\begin{aligned}
J & =x_{\xi} y_{\eta} z_{\zeta}+y_{\xi} z_{\eta} x_{\zeta}+z_{\xi} x_{\eta} y_{\zeta}-x_{\xi} y_{\zeta} z_{\eta}-y_{\xi} z_{\zeta} x_{\eta}-z_{\xi} x_{\zeta} y_{\eta} \\
& =\left(\xi_{x} \eta_{y} \zeta_{z}+\xi_{y} \eta_{z} \zeta_{x}+\xi_{z} \eta_{x} \zeta_{y}-\xi_{x} \eta_{z} \zeta_{y}-\xi_{y} \eta_{x} \zeta_{z}-\xi_{z} \eta_{y} \zeta_{x}\right)^{-1}
\end{aligned}
$$

Invariants of the transformation matrix can be derived

$$
\begin{aligned}
& \frac{\partial J \xi_{x}}{\partial \xi}+\frac{\partial J \eta_{x}}{\partial \eta}+\frac{\partial J \zeta_{x}}{\partial \zeta}=0 \\
& \frac{\partial J \xi_{y}}{\partial \xi}+\frac{\partial J \eta_{y}}{\partial \eta}+\frac{\partial J \zeta_{y}}{\partial \zeta}=0 \\
& \frac{\partial J \xi_{z}}{\partial \xi}+\frac{\partial J \eta_{z}}{\partial \eta}+\frac{\partial J \zeta_{z}}{\partial \zeta}=0
\end{aligned}
$$

that are used in the formulation of the conservative form of the transformed equation. For a detailed discussion on the transformation please refer to [123]. 


\section{Appendix E}

\section{Equations in Curvilinear Coordinates}

The conservation equations given in appendix $\mathrm{C}$ transformed using the rules in appendix $\mathrm{D}$ into the curvilinear coordinates $\xi, \eta, \zeta$ are

$$
\frac{\partial \hat{\vec{Q}}}{\partial t}+\frac{\partial \hat{\vec{E}}_{1}}{\partial \xi}+\frac{\partial \hat{\vec{E}}_{2}}{\partial \eta}+\frac{\partial \hat{\vec{E}}_{3}}{\partial \zeta}=\hat{S}
$$

with the transformed conservative variables $\hat{\vec{Q}}$, the transformed flux vectors $\hat{\vec{E}}_{1}, \hat{\vec{E}}_{2}, \hat{\vec{E}}_{3}$ and the source term $\hat{\vec{S}}$

$$
\begin{aligned}
\hat{\vec{Q}} & =J \vec{Q} \\
\hat{\vec{E}}_{1} & =J\left(\xi_{x} \vec{F}_{1}+\xi_{y} \vec{F}_{2}+\xi_{z} \vec{F}_{3}\right) \\
\hat{\vec{E}}_{2} & =J\left(\eta_{x} \vec{F}_{1}+\eta_{y} \vec{F}_{2}+\eta_{z} \vec{F}_{3}\right) \\
\hat{\vec{E}}_{3} & =J\left(\zeta_{x} \vec{F}_{1}+\zeta_{y} \vec{F}_{2}+\zeta_{z} \overrightarrow{F_{3}}\right) \\
\hat{S} & =J \vec{S}
\end{aligned}
$$

calculated as

$$
\begin{gathered}
\hat{\vec{E}}_{1}=J\left(\begin{array}{c}
\bar{\rho} U \\
\bar{\rho} U \tilde{u}+\xi_{x} \bar{p} \\
\bar{\rho} U \tilde{v}+\xi_{y} \bar{p} \\
\bar{\rho} U \tilde{w}+\xi_{z} \bar{p} \\
U(\bar{\rho} \tilde{e}+\bar{p}) \\
\bar{\rho} \check{\nu} U
\end{array}\right)-\frac{J}{R e_{0}}\left(\begin{array}{c}
0 \\
\xi_{x} \tau_{x x}+\xi_{y} \tau_{x y}+\xi_{z} \tau_{x z} \\
\xi_{x} \tau_{x y}+\xi_{y} \tau_{y y}+\xi_{z} \tau_{y z} \\
\xi_{x} \tau_{x z}+\xi_{y} \tau_{y z}+\xi_{z} \tau_{z z} \\
\xi_{x} E_{1_{D_{5}}}+\xi_{y} E_{1 D_{5}}+\xi_{z} E_{1_{D_{5}}} \\
(\bar{\mu}+\sigma \bar{\rho} \check{\nu})\left(\xi_{x} \check{\nu}_{x}+\xi_{y} \check{\nu}_{y}+\xi_{z} \check{\nu}_{z}\right)
\end{array}\right) \\
\hat{\vec{E}}_{2}=J\left(\begin{array}{c}
0 \\
\bar{\rho} V \\
\bar{\rho} V \tilde{u}+\eta_{x} \bar{p} \\
\bar{\rho} V \tilde{v}+\eta_{y} \bar{p} \\
\bar{\rho} V \tilde{w}+\eta_{z} \bar{p} \\
V(\bar{\rho} \tilde{e}+\bar{p}) \\
\bar{\rho} \check{\nu} V
\end{array}\right)-\frac{J}{R e_{0}}\left(\begin{array}{c}
\eta_{x} \tau_{x x}+\eta_{y} \tau_{x y}+\eta_{z} \tau_{x z} \\
\eta_{x} \tau_{x y}+\eta_{y} \tau_{y y}+\eta_{z} \tau_{y z} \\
\eta_{x} \tau_{x z}+\eta_{y} \tau_{y z}+\eta_{z} \tau_{z z} \\
\eta_{x} E_{2_{D_{5}}}+\eta_{y} E_{2_{D_{5}}}+\eta_{z} E_{2_{D_{5}}} \\
(\bar{\mu}+\sigma \bar{\rho} \check{\nu})\left(\eta_{x} \check{\nu}_{x}+\eta_{y} \check{\nu}_{y}+\eta_{z} \check{\nu}_{z}\right)
\end{array}\right)
\end{gathered}
$$




$$
\hat{\vec{E}}_{3}=J\left(\begin{array}{c}
\bar{\rho} W \\
\bar{\rho} W \tilde{u}+\zeta_{x} \bar{p} \\
\bar{\rho} W \tilde{v}+\zeta_{y} \bar{p} \\
\bar{\rho} W \tilde{w}+\zeta_{z} \bar{p} \\
W(\bar{\rho} \tilde{e}+\bar{p}) \\
\bar{\rho} \check{\nu} W
\end{array}\right)-\frac{J}{R e_{0}}\left(\begin{array}{c}
0 \\
\zeta_{x} \tau_{x x}+\zeta_{y} \tau_{x y}+\zeta_{z} \tau_{x z} \\
\zeta_{x} \tau_{x y}+\zeta_{y} \tau_{y y}+\zeta_{z} \tau_{y z} \\
\zeta_{x} \tau_{x z}+\zeta_{y} \tau_{y z}+\zeta_{z} \tau_{z z} \\
\zeta_{x} E_{3_{D_{5}}}+\zeta_{y} E_{3_{D_{5}}}+\zeta_{z} E_{3_{D_{5}}} \\
(\bar{\mu}+\sigma \bar{\rho} \check{\nu})\left(\zeta_{x} \check{\nu}_{x}+\zeta_{y} \check{\nu}_{y}+\zeta_{z} \check{\nu}_{z}\right)
\end{array}\right)
$$

with

$$
\begin{aligned}
& E_{1_{D_{5}}}=\tilde{u} \tau_{x x}+\tilde{v} \tau_{x y}+\tilde{w} \tau_{x z}+q_{x} \\
& E_{2_{D_{5}}}=\tilde{u} \tau_{x y}+\tilde{v} \tau_{y y}+\tilde{w} \tau_{y z}+q_{y} \\
& E_{3_{D_{5}}}=\tilde{u} \tau_{x z}+\tilde{v} \tau_{y z}+\tilde{w} \tau_{z z}+q_{z}
\end{aligned}
$$

and the contravariant velocities $U, V, W$

$$
\begin{aligned}
U & =\xi_{x} \tilde{u}+\xi_{y} \tilde{v}+\xi_{z} \tilde{w} \\
V & =\eta_{x} \tilde{u}+\eta_{y} \tilde{v}+\eta_{z} \tilde{w} \\
W & =\zeta_{x} \tilde{u}+\zeta_{y} \tilde{v}+\zeta_{z} \tilde{w}
\end{aligned}
$$

The stress tensor and heat conduction are derived accordingly

$$
\begin{gathered}
\tau_{x x}=\frac{2}{3}\left(\bar{\mu}+\mu_{t}\right)\left[2\left(\xi_{x} \tilde{u}_{\xi}+\eta_{x} \tilde{u}_{\eta}+\zeta_{x} \tilde{u}_{\zeta}\right)-\left(\xi_{y} \tilde{v}_{\xi}+\eta_{y} \tilde{v}_{\eta}+\zeta_{y} \tilde{v}_{\zeta}+\xi_{z} \tilde{w}_{\xi}+\eta_{z} \tilde{w}_{\eta}+\zeta_{z} \tilde{w}_{\zeta}\right)\right] \\
\tau_{y y}=\frac{2}{3}\left(\bar{\mu}+\mu_{t}\right)\left[2\left(\xi_{y} \tilde{v}_{\xi}+\eta_{y} \tilde{v}_{\eta}+\zeta_{y} \tilde{v}_{\zeta}\right)-\left(\xi_{x} \tilde{u}_{\xi}+\eta_{x} \tilde{u}_{\eta}+\zeta_{x} \tilde{u}_{\zeta}+\xi_{z} \tilde{w}_{\xi}+\eta_{z} \tilde{w}_{\eta}+\zeta_{z} \tilde{w}_{\zeta}\right)\right] \\
\tau_{z z}=\frac{2}{3}\left(\bar{\mu}+\mu_{t}\right)\left[2\left(\xi_{z} \tilde{w}_{\xi}+\eta_{z} \tilde{w}_{\eta}+\zeta_{z} \tilde{w}_{\zeta}\right)-\left(\xi_{x} \tilde{u}_{\xi}+\eta_{x} \tilde{u}_{\eta}+\zeta_{x} \tilde{u}_{\zeta}+\xi_{y} \tilde{v}_{\xi}+\eta_{y} \tilde{v}_{\eta}+\zeta_{y} \tilde{v}_{\zeta}\right)\right] \\
\tau_{x y}=\tau_{y x}=\left(\bar{\mu}+\mu_{t}\right)\left(\xi_{y} \tilde{u}_{\xi}+\eta_{y} \tilde{u}_{\eta}+\zeta_{y} \tilde{u}_{\zeta}+\xi_{x} \tilde{v}_{\xi}+\eta_{x} \tilde{v}_{\eta}+\zeta_{x} \tilde{v}_{\zeta}\right) \\
\tau_{x z}=\tau_{z x}=\left(\bar{\mu}+\mu_{t}\right)\left(\xi_{z} \tilde{u}_{\xi}+\eta_{z} \tilde{u}_{\eta}+\zeta_{z} \tilde{u}_{\zeta}+\xi_{x} \tilde{w}_{\xi}+\eta_{x} \tilde{w}_{\eta}+\zeta_{x} \tilde{w}_{\zeta}\right) \\
\tau_{y z}=\tau_{z y}=\left(\bar{\mu}+\mu_{t}\right)\left(\xi_{z} \tilde{v}_{\xi}+\eta_{z} \tilde{v}_{\eta}+\zeta_{z} \tilde{v}_{\zeta}+\xi_{y} \tilde{w}_{\xi}+\eta_{y} \tilde{w}_{\eta}+\zeta_{y} \tilde{w}_{\zeta}\right) \\
q_{x}=\frac{1}{\left(\gamma_{0}-1\right)}\left(\frac{\bar{\mu}}{P r_{0}}+\frac{\mu_{t}}{P r_{t}}\right)\left(\xi_{x} T_{\xi}+\eta_{x} T_{\eta}+\zeta_{x} T_{\zeta}\right) \\
q_{y}=\frac{1}{\left(\gamma_{0}-1\right)}\left(\frac{\bar{\mu}}{P r_{0}}+\frac{\mu_{t}}{P r_{t}}\right)\left(\xi_{y} T_{\xi}+\eta_{y} T_{\eta}+\zeta_{y} T_{\zeta}\right) \\
q_{z}=\frac{1}{\left(\gamma_{0}-1\right)}\left(\frac{\bar{\mu}}{P r_{0}}+\frac{\mu_{t}}{P r_{t}}\right)\left(\xi_{z} T_{\xi}+\eta_{z} T_{\eta}+\zeta_{z} T_{\zeta}\right)
\end{gathered}
$$

Other derivatives in the source term $\hat{\vec{S}}$ are not given explicitly here and can be derived easily following the general transformation rule for a quantity $\Phi$

$$
\begin{aligned}
& \Phi_{x}=\xi_{x} \Phi_{\xi}+\eta_{x} \Phi_{\eta}+\zeta_{x} \Phi_{\zeta} \\
& \Phi_{y}=\xi_{y} \Phi_{\xi}+\eta_{y} \Phi_{\eta}+\zeta_{y} \Phi_{\zeta} \\
& \Phi_{z}=\xi_{z} \Phi_{\xi}+\eta_{z} \Phi_{\eta}+\zeta_{z} \Phi_{\zeta}
\end{aligned}
$$




\section{Appendix F}

\section{Discretization of Advective Terms according to the $A U S M$ Scheme}

The total advective flux $\vec{F}_{A}$ is divided into a convective part $\vec{F}_{C}$ and a pressure part $\vec{F}_{P}$, whereas the energy equation is rewritten in terms of the total enthalpy $H=e+p / \rho$ and the local contravariant $\mathrm{MACH}$ number $\mathcal{M}=U / a$ is introduced. The scheme will be exemplary presented in curvilinear coordinates for the flux $\hat{\vec{E}}_{A_{1}}$ in the direction $\xi^{1}$

$$
\hat{\vec{E}}_{A_{1}}=J\left(\begin{array}{c}
\rho U \\
\rho U u+\xi_{x} p \\
\rho U v+\xi_{y} p \\
\rho U w+\xi_{z} p \\
U(\rho e+p) \\
\rho U \check{\nu}
\end{array}\right)=\underbrace{J \mathcal{M}\left(\begin{array}{c}
\rho a \\
\rho a u \\
\rho a v \\
\rho a w \\
\rho a H \\
\rho a \check{\nu}
\end{array}\right)}_{\hat{\vec{E}}_{C_{1}}}+\underbrace{J\left(\begin{array}{c}
0 \\
\xi_{x} p \\
\xi_{y} p \\
\xi_{z} p \\
0 \\
0
\end{array}\right)}_{\hat{\vec{E}}_{P_{1}}} .
$$

The numerical flux at a cell face $i+1 / 2$ of the convective part $\hat{\vec{E}}_{C_{1}}^{i+1 / 2}$ is calculated in terms of left $\left.{ }_{L}\right)$ and right $\left.{ }_{R}\right)$ interface values of $\vec{\Phi}=(\rho a, \rho a u, \rho a v, \rho a w, \rho a H, \rho a \check{\nu})^{T}$ according to

$$
\hat{\vec{E}}_{C_{1}}^{i+1 / 2}=\frac{J}{2}\left[\mathcal{M}_{1 / 2}^{i+1 / 2}\left(\vec{\Phi}_{L}^{i+1 / 2}+\vec{\Phi}_{R}^{i+1 / 2}\right)-\left|\mathcal{M}_{1 / 2}^{i+1 / 2}\right|\left(\vec{\Phi}_{R}^{i+1 / 2}-\vec{\Phi}_{L}^{i+1 / 2}\right)\right],
$$

where the splitted contravariant $\mathrm{MACH}$ number on the cell face $\mathcal{M}_{1 / 2}^{i+1 / 2}$ is given by

$$
\mathcal{M}_{1 / 2}^{i+1 / 2}=\left(\mathcal{M}_{L}^{i+1 / 2}\right)^{+}+\left(\mathcal{M}_{R}^{i+1 / 2}\right)^{-}
$$

\footnotetext{
${ }^{1}$ the other directions are treated accordingly.
} 
and the forward ()$^{+}$and backward ()$^{-}$values calculated as

$$
\begin{aligned}
& \left(\mathcal{M}_{L}^{i+1 / 2}\right)^{+}= \begin{cases}\frac{\breve{\alpha}_{L}\left(\frac{\left(\mathcal{M}_{L}^{i+1 / 2}+1\right)^{2}}{4}-\frac{\mathcal{M}_{L}^{i+1 / 2}+\left|\mathcal{M}_{L}^{i+1 / 2}\right|}{2}\right)+\left|\mathcal{M}_{L}^{i+1 / 2}\right|}{2} & \text { if }\left|\mathcal{M}_{L}^{i+1 / 2}\right| \leq 1 \\
\frac{\mathcal{M}_{L}^{i+1 / 2}+\left|\mathcal{M}_{L}^{i+1 / 2}\right|}{2} & \text { otherwise }\end{cases} \\
& \left(\mathcal{M}_{R}^{i+1 / 2}\right)^{-}= \begin{cases}\frac{\breve{\alpha}_{R}\left(-\frac{\left(\mathcal{M}_{R}^{i+1 / 2}-1\right)^{2}}{4}-\frac{\mathcal{M}_{R}^{i+1 / 2}-\left|\mathcal{M}_{R}^{i+1 / 2}\right|}{2}\right)+}{2} & \text { if }\left|\mathcal{M}_{R}^{i+1 / 2}\right| \leq 1 \\
\frac{\mathcal{M}_{R}^{i+1 / 2}-\left|\mathcal{M}_{R}^{i+1 / 2}\right|}{2}-\left|\mathcal{M}_{R}^{i+1 / 2}\right| & \text { otherwise }\end{cases}
\end{aligned}
$$

with

$$
\breve{\alpha}_{L}=\left.\frac{2(p / \rho)_{L}}{(p / \rho)_{L}+(p / \rho)_{R}}\right|^{i+1 / 2} \quad \breve{\alpha}_{R}=\left.\frac{2(p / \rho)_{R}}{(p / \rho)_{L}+(p / \rho)_{R}}\right|^{i+1 / 2}
$$

and

$$
\mathcal{M}_{L}^{i+1 / 2}=\frac{U_{L}^{i+1 / 2}}{a_{m}^{i+1 / 2}} \quad \mathcal{M}_{R}^{i+1 / 2}=\frac{U_{R}^{i+1 / 2}}{a_{m}^{i+1 / 2}} \quad a_{m}^{i+1 / 2}=\sqrt{a_{L}^{i+1 / 2} a_{R}^{i+1 / 2}}
$$

The pressure part of the flux is calculated according to

$$
\hat{\vec{E}}_{P_{1}}^{i+1 / 2}=J\left(\begin{array}{c}
0 \\
\xi_{x}^{i+1 / 2} p_{1 / 2}^{i+1 / 2} \\
\xi_{y}^{i+1 / 2} p_{1 / 2}^{i+1 / 2} \\
\xi_{z}^{i+1 / 2} p_{1 / 2}^{i+1 / 2} \\
0 \\
0
\end{array}\right) \quad \text { with } \quad p_{1 / 2}^{i+1 / 2}=\left(p_{L}^{i+1 / 2}\right)^{+}+\left(p_{R}^{i+1 / 2}\right)^{-}
$$

calculated by

$$
\begin{aligned}
& \left(p_{L}^{i+1 / 2}\right)^{+}=\left\{\begin{array}{lc}
p_{L}^{i+1 / 2} \frac{\left(\mathcal{M}_{L}^{i+1 / 2}+1\right)^{2}\left(2-\mathcal{M}_{L}^{i+1 / 2}\right)}{4} & \text { if }\left|\mathcal{M}_{L}^{i+1 / 2}\right| \leq 1 \\
p_{L}^{i+1 / 2} \frac{\mathcal{M}_{L}^{i+1 / 2}+\left|\mathcal{M}_{L}^{i+1 / 2}\right|}{2 \mathcal{M}_{L}^{i+1 / 2}} & \text { otherwise }
\end{array}\right. \\
& \left(p_{R}^{i+1 / 2}\right)^{-}= \begin{cases}p_{R}^{i+1 / 2} \frac{\left(\mathcal{M}_{R}^{i+1 / 2}-1\right)^{2}\left(2+\mathcal{M}_{R}^{i+1 / 2}\right)}{4} & \text { if }\left|\mathcal{M}_{R}^{i+1 / 2}\right| \leq 1 \\
p_{R}^{i+1 / 2} \frac{\mathcal{M}_{R}^{i+1 / 2}-\left|\mathcal{M}_{R}^{i+1 / 2}\right|}{2 \mathcal{M}_{R}^{i+1 / 2}} & \text { otherwise }\end{cases}
\end{aligned}
$$


The $A U S M D$ and $A U S M V$ versions have a behavior similar $F D S$ and $F V S$, respectively, and can be combined to give the $A U S M D V$ version, which involves a different calculation than (F.2) for the mass flux $\vec{\Psi}=(\rho U u, \rho U v, \rho U w)^{T}$ in the momentum equations:

$A U S M D$

$$
\vec{\Psi}_{A U S M D}^{i+1 / 2}=\frac{1}{2}\left[(\rho U)_{1 / 2}^{i+1 / 2}\left(\vec{u}_{L}^{i+1 / 2}+\vec{u}_{R}^{i+1 / 2}\right)-\left|(\rho U)_{1 / 2}^{i+1 / 2}\right|\left(\vec{u}_{R}^{i+1 / 2}-\vec{u}_{L}^{i+1 / 2}\right)\right]
$$

$A U S M V$

$$
\vec{\Psi}_{A U S M V}^{i+1 / 2}=\left(\mathcal{M}_{L}^{i+1 / 2}\right)^{+} a_{L}(\rho \vec{u})_{L}^{i+1 / 2}+\left(\mathcal{M}_{R}^{i+1 / 2}\right)^{-} a_{R}(\rho \vec{u})_{R}^{i+1 / 2}
$$

$A U S M D V$

$$
\vec{\Psi}_{A U S M D V}^{i+1 / 2}=\left(\frac{1}{2}+s\right) \vec{\Psi}_{A U S M V}^{i+1 / 2}+\left(\frac{1}{2}-s\right) \vec{\Psi}_{A U S M D}^{i+1 / 2}
$$

where $(\rho U)_{1 / 2}^{i+1 / 2}=\left.\rho_{L} U_{L}^{+}\right|^{i+1 / 2}+\left.\rho_{R} U_{R}^{-}\right|^{i+1 / 2}$ and $s$ is a switching function of the pressure gradient

$$
s=\min \left\{1,10 \max \left(\left|\frac{p^{i}-p^{i-1}}{\min \left(p^{i}, p^{i-1}\right)}\right|,\left|\frac{p^{i+1}-p^{i}}{\min \left(p^{i+1}, p^{i}\right)}\right|,\left|\frac{p^{i+2}-p^{i+1}}{\min \left(p^{i+2}, p^{i+1}\right)}\right|\right)\right\}
$$

The reconstruction of left and right values is performed according to the classical $M U S C L$ approach to achieve a second-order accuracy

$$
\begin{aligned}
& \Phi_{L}^{i+1 / 2}=\Phi^{i}+\frac{l^{i+1 / 2}}{\left(l^{i-1 / 2}+l^{i+1 / 2}\right)^{2}}\left[l^{i-1 / 2}\left(\Phi^{i+1}-\Phi^{i}\right)+l^{i+1 / 2}\left(\Phi^{i}-\Phi^{i-1}\right)\right] \\
& \Phi_{R}^{i+1 / 2}=\Phi^{i+1}-\frac{l^{i+3 / 2}}{\left(l^{i+1 / 2}+l^{i+3 / 2}\right)^{2}}\left[l^{i+1 / 2}\left(\Phi^{i+2}-\Phi^{i+1}\right)+l^{i+3 / 2}\left(\Phi^{i+1}-\Phi^{i}\right)\right](
\end{aligned}
$$

or a third order accuracy

$$
\begin{aligned}
\Phi_{L}^{i+1 / 2}= & \frac{1}{4 l^{i-3 / 2}\left(l^{i-3 / 2}+l^{i+1 / 2}\right)}\left[\left(l^{i-3 / 2} l^{i+1 / 2}+2\left(l^{i-3 / 2}\right)^{2}\right) \Phi^{i+1}\right. \\
& \left.+\left(2\left(l^{i-3 / 2}\right)^{2}+3 l^{i-3 / 2} l^{i+1 / 2}+\left(l^{i+1 / 2}\right)^{2}\right) \Phi^{i}-\left(l^{i+1 / 2}\right)^{2} \Phi^{i-1}\right] \\
\Phi_{R}^{i+1 / 2}= & \frac{1}{4 l^{i+3 / 2}\left(l^{i+3 / 2}+l^{i+1 / 2}\right)}\left[\left(l^{i+3 / 2} l^{i+1 / 2}+2\left(l^{i+3 / 2}\right)^{2}\right) \Phi^{i}\right. \\
& \left.+\left(2\left(l^{i+3 / 2}\right)^{2}+3 l^{i+3 / 2} l^{i+1 / 2}+\left(l^{i+1 / 2}\right)^{2}\right) \Phi^{i+1}-\left(l^{i+1 / 2}\right)^{2} \Phi^{i+2}\right]
\end{aligned}
$$

with the arc length $l^{i+1 / 2}$ defined as

$$
l^{i+1 / 2}=\sqrt{\left(x^{i+1}-x^{i}\right)^{2}+\left(y^{i+1}-y^{i}\right)^{2}+\left(z^{i+1}-z^{i}\right)^{2}} .
$$




\section{Appendix G}

\section{Characteristical Form of the EULER Equations}

The conservation equations in curvilinear coordinates (E.1) are simplified for inviscid flows by neglecting the diffusive terms and rewritten in terms of the primitive variables $\rho, \vec{v}, p$

$$
\begin{gathered}
\hat{\vec{O}}=\left(\begin{array}{c}
\rho \\
u \\
v \\
w \\
p
\end{array}\right) \\
\frac{\partial \hat{\vec{O}}}{\partial t}+\hat{A}_{1} \frac{\partial \hat{\vec{O}}}{\partial \xi}+\hat{A}_{2} \frac{\partial \hat{\vec{O}}}{\partial \eta}+\hat{A}_{3} \frac{\partial \hat{\vec{O}}}{\partial \zeta}=0
\end{gathered}
$$

with the matrices $\hat{A}_{1}, \hat{A}_{2}, \hat{A}_{3}$ containing the flux jacobians

$$
\begin{aligned}
\hat{A}_{1}= & \left(\begin{array}{ccccc}
U & \rho \xi_{x} & \rho \xi_{y} & \rho \xi_{z} & 0 \\
0 & U & 0 & 0 & \xi_{x} / \rho \\
0 & 0 & U & 0 & \xi_{y} / \rho \\
0 & 0 & 0 & U & \xi_{z} / \rho \\
0 & \xi_{x} \rho a^{2} & \xi_{y} \rho a^{2} & \xi_{z} \rho a^{2} & U
\end{array}\right) \\
\hat{A}_{2}= & \left(\begin{array}{ccccc}
V & \rho \eta_{x} & \rho \eta_{y} & \rho \eta_{z} & 0 \\
0 & V & 0 & 0 & \eta_{x} / \rho \\
0 & 0 & V & 0 & \eta_{y} / \rho \\
0 & 0 & 0 & V & \eta_{z} / \rho \\
0 & \eta_{x} \rho a^{2} & \eta_{y} \rho a^{2} & \eta_{z} \rho a^{2} & V
\end{array}\right) \\
\hat{A}_{3}= & \left(\begin{array}{ccccc}
W & \rho \zeta_{x} & \rho \zeta_{y} & \rho \zeta_{z} & 0 \\
0 & W & 0 & 0 & \zeta_{x} / \rho \\
0 & 0 & W & 0 & \zeta_{y} / \rho \\
0 & 0 & 0 & W & \zeta_{z} / \rho \\
0 & \zeta_{x} \rho a^{2} & \zeta_{y} \rho a^{2} & \zeta_{z} \rho a^{2} & W
\end{array}\right) .
\end{aligned}
$$

The matrices $\hat{A}_{1}, \hat{A}_{2}, \hat{A}_{3}$ have a similar form and can be interchanged by changing the metric terms. For the one-dimensional analysis it suffices to consider only one direction, e.g. the $\xi$ direction. The matrix $\hat{A}_{1}$ can be diagonalized through the eigenvalue matrix $\hat{\Lambda}_{1}$ and the 
eigenvector matrix $\hat{M}_{1}$

$$
\begin{aligned}
& \frac{\partial \hat{\vec{O}}}{\partial t}+\hat{A}_{1} \frac{\partial \hat{\vec{O}}}{\partial \xi}=0 \\
& \hat{A}_{1}=\hat{M}_{1} \hat{\Lambda}_{1} \hat{M}_{1}^{-1}
\end{aligned}
$$

with

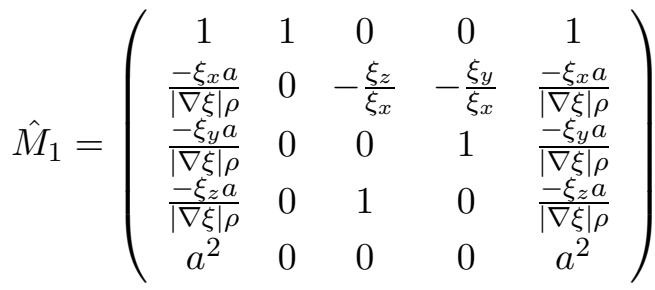

$$
\begin{aligned}
& \hat{M}_{1}^{-1}=\left(\begin{array}{ccccc}
0 & -\frac{1}{2} \frac{\xi_{x} \rho}{|\nabla \xi| a} & -\frac{1}{2} \frac{\xi_{y} \rho}{|\nabla \xi| a} & -\frac{1}{2} \frac{\xi_{z} \rho}{|\nabla \xi| a} & \frac{1}{2} \frac{1}{a^{2}} \\
1 & 0 & 0 & 0 & -\frac{1}{a^{2}} \\
0 & \frac{-\xi_{z} \xi_{x}}{|\nabla \xi|^{2}} & \frac{-\xi_{z} \xi_{y}}{|\nabla \xi|^{2}} & \frac{\xi_{y}^{2}+\xi_{x}^{2}}{|\nabla \xi|^{2}} & 0 \\
0 & \frac{-\xi_{x} \xi_{x}}{|\nabla \xi|^{2}} & \frac{\xi_{\xi}^{2}+\xi_{x}^{2}}{|\nabla \xi|^{2}} & \frac{-\xi_{y} \xi_{y}}{|\nabla \xi|^{2}} & 0 \\
0 & \frac{1}{2} \frac{\xi_{x} \rho}{\nabla \nabla \xi \mid a} & \frac{1}{2} \frac{\xi_{y} \rho}{\nabla \nabla \xi \mid a} & \frac{1}{2} \frac{\xi_{z} \rho}{\nabla \nabla \xi \mid a} & \frac{1}{2} \frac{1}{a^{2}}
\end{array}\right)
\end{aligned}
$$

and $\hat{\Lambda}_{1}=\operatorname{diag}\left(\lambda_{1}, \lambda_{2}, \lambda_{3}, \lambda_{4}, \lambda_{5}\right)$ defined as

$$
\begin{aligned}
& \lambda_{1}=\xi_{x} u+\xi_{y} v+\xi_{z} w-a|\nabla \xi|=U-a|\nabla \xi| \\
& \lambda_{2}=\xi_{x} u+\xi_{y} v+\xi_{z} w=U \\
& \lambda_{3}=\xi_{x} u+\xi_{y} v+\xi_{z} w=U \\
& \lambda_{4}=\xi_{x} u+\xi_{y} v+\xi_{z} w=U \\
& \lambda_{5}=\xi_{x} u+\xi_{y} v+\xi_{z} w+a|\nabla \xi|=U+a|\nabla \xi|
\end{aligned}
$$

with the metric term

$$
|\nabla \xi|=\sqrt{\xi_{x}^{2}+\xi_{y}^{2}+\xi_{z}^{2}} .
$$

By left multiplying equation (G.6) with $\hat{M}_{1}^{-1}$ which is assumed to have constant entries denoted by the subscript $*$ the characteristic form of the EuLER equations are derived

$$
\frac{\partial \hat{\vec{C}}}{\partial t}+\underbrace{\hat{\Lambda}_{1} \frac{\partial \hat{\vec{C}}}{\partial \xi}}_{\mathcal{L}_{i}}=0
$$

with the vector of the characteristic variables $\hat{\vec{C}}$ defined as

$$
\hat{\vec{C}}=\hat{M}_{1}^{-1} \hat{\vec{O}}=\left(\begin{array}{c}
\frac{1}{2}\left(p-\frac{\rho_{*} a_{*} U}{|\nabla \xi|}\right) \\
\rho-\frac{p}{a_{*}^{2}} \\
\frac{v\left(\xi_{x}^{2}+\xi_{z}^{2}\right)-\xi_{y}\left(\xi_{x} u+\xi_{z} w\right)}{|\nabla \xi|^{2}} \\
\frac{w\left(\xi_{x}^{2}+\xi_{y}^{2}\right)-\xi_{z}\left(\xi_{x} u+\xi_{y} v\right)}{|\nabla \xi|^{2}} \\
\frac{1}{2}\left(p+\frac{\rho_{*} a_{*} U}{|\nabla \xi|}\right)
\end{array}\right) .
$$


The product of eigenvalues $\lambda_{i}$ of equation (G.10) with the derivative $\frac{\partial \hat{\vec{C}}}{\partial \xi}$ are calculated as

$$
\begin{aligned}
& \mathcal{L}_{1}=\lambda_{1} \frac{1}{2}\left[\frac{\partial p}{\partial \xi}-\frac{\rho_{*} a_{*}}{|\nabla \xi|}\left(\xi_{x} \frac{\partial u}{\partial \xi}+\xi_{y} \frac{\partial v}{\partial \xi}+\xi_{z} \frac{\partial w}{\partial \xi}\right)\right] \\
& \mathcal{L}_{2}=\lambda_{2}\left[\frac{\partial \rho}{\partial \xi}-\frac{1}{a_{*}^{2}} \frac{\partial p}{\partial \xi}\right] \\
& \mathcal{L}_{3}=\lambda_{3}\left[\frac{\xi_{x}^{2}+\xi_{z}^{2}}{|\nabla \xi|^{2}} \frac{\partial v}{\partial \xi}-\frac{\xi_{y} \xi_{x}}{|\nabla \xi|^{2}} \frac{\partial u}{\partial \xi}-\frac{\xi_{y} \xi_{z}}{|\nabla \xi|^{2}} \frac{\partial w}{\partial \xi}\right] \\
& \mathcal{L}_{4}=\lambda_{4}\left[\frac{\xi_{x}^{2}+\xi_{y}^{2}}{|\nabla \xi|^{2}} \frac{\partial w}{\partial \xi}-\frac{\xi_{z} \xi_{x}}{|\nabla \xi|^{2}} \frac{\partial u}{\partial \xi}-\frac{\xi_{z} \xi_{y}}{|\nabla \xi|^{2}} \frac{\partial v}{\partial \xi}\right] \\
& \mathcal{L}_{5}=\lambda_{5} \frac{1}{2}\left[\frac{\partial p}{\partial \xi}+\frac{\rho_{*} a_{*}}{|\nabla \xi|}\left(\xi_{x} \frac{\partial u}{\partial \xi}+\xi_{y} \frac{\partial v}{\partial \xi}+\xi_{z} \frac{\partial w}{\partial \xi}\right)\right] .
\end{aligned}
$$

The local one-dimensional inviscid $L O D I$ relations conveying the time derivative of the primitive variables in terms of $\mathcal{L}_{i}$ can then be given

$$
\begin{aligned}
& \left.\frac{\partial p}{\partial t}\right|_{\xi}=-\frac{1}{2}\left(\mathcal{L}_{1}+\mathcal{L}_{5}\right) \\
& \left.\frac{\partial \rho}{\partial t}\right|_{\xi}=\frac{1}{2 a_{*}^{2}}\left(\mathcal{L}_{1}+\mathcal{L}_{5}\right)-\mathcal{L}_{2} \\
& \left.\frac{\partial u}{\partial t}\right|_{\xi}=\frac{\xi_{x}}{2 \rho_{*} a_{*}|\nabla \xi|}\left(\mathcal{L}_{1}-\mathcal{L}_{5}\right)+\frac{\xi_{y}}{\xi_{x}} \mathcal{L}_{3}+\frac{\xi_{z}}{\xi_{x}} \mathcal{L}_{4} \\
& \left.\frac{\partial v}{\partial t}\right|_{\xi}=\frac{\xi_{y}}{2 \rho_{*} a_{*}|\nabla \xi|}\left(\mathcal{L}_{1}-\mathcal{L}_{5}\right)-\mathcal{L}_{3} \\
& \left.\frac{\partial w}{\partial t}\right|_{\xi}=\frac{\xi_{z}}{2 \rho_{*} a_{*}|\nabla \xi|}\left(\mathcal{L}_{1}-\mathcal{L}_{5}\right)-\mathcal{L}_{4} .
\end{aligned}
$$




\section{Curriculum Vitae}

Name :

12. Juli 1973

$1978-1983$

$1983-1992$

$1992-1994$

$1994-1997$

$1995-1997$

seit 1997
Ehab Mahmoud Aziz Mohamed Fares

geboren in El-Menia, Ägypten als Sohn von Mahmoud Aziz Fares und Azza Ibrahim Esmat

Besuch mehrerer Grundschulen in Riyadh (Saudiarabien), El-Ain (Emiraten), Alexandria (Ägypten) und Giza (Ägypten)

Besuch der Deutschen Evangelischen Oberschule in Giza, Ägypten, Abschluß deutsche Hochschulreife

Studium des Maschinenbaus an der TU Braunschweig, Abschluß Vordiplom

Studium des Maschinenbaus an der RWTH Aachen, Verteifungsrichtung Luft- und Raumfahrt, Abschluß Diplom

Wissenschaftliche Hilfskraft am Intitut für Luft- und Raumfahrt der RWTH Aachen

Wissenschaftlicher Angestellter am Aerodynamischen Institut der RWTH Aachen 